\title{
Acknowledgment to Reviewers of Sensors in 2021
}

\author{
Sensors Editorial Office
}

Citation: Sensors Editorial Office. Acknowledgment to Reviewers of Sensors in 2021. Sensors 2022, 22, 1052. https://doi.org/10.3390/ s22031052

Published: 29 January 2022

Publisher's Note: MDPI stays neutral with regard to jurisdictional claims in published maps and institutional affiliations.

Copyright: (C 2022 by the author. Licensee MDPI, Basel, Switzerland. This article is an open access article distributed under the terms and conditions of the Creative Commons Attribution (CC BY) license (https://creativecommons.org/licenses/by/4.0/).

MDPI AG, St. Alban-Anlage 66, 4052 Basel, Switzerland

Rigorous peer-reviews are the basis of high-quality academic publishing. Thanks to the great efforts of our reviewers, Sensors was able to maintain its standards for the high quality of its published papers. Thanks to the contribution of our reviewers, in 2021, the median time to first decision was 16 days and the median time to publication was 40 days. The editors would like to extend their gratitude and recognition to the following reviewers for their precious time and dedication, regardless of whether the papers they reviewed were finally published:

A. D. Prasad

A. Dan Wilson

A.S.M. Kayes

A.T. Ezhil Vilian

Aakanksha Tewari

Aarón Ángel Salas-Sánchez

Aaron J. Pung

Aashish Priye

Abas Sabouni

Abayomi Otebolaku

Abbas Abbaszadeh Shahri

Abbas Acar

Abbas Haddadi

Abbas Madani

Abbas Orand

Abbas Shafiee

Abbas Shahri

Abbass Nasser

Abd Krim Seghouane

Abdallah Moubayed

Abdel F. Isakovic

Abdel Fattah Sheta

Abdeldjalil Ouahabi

Abdelfateh Kerrouche

Abdelhak Merizig

Abdelhameed Fawzy

Abdelhamied Ashraf

Abdelkader Nasreddine Belkacem

Abdelkrim Rebiai

Abdellah Chehri

Abdellatif Akjouj

Abdellatif Zaidi

Abdelmalek Bouguettaya

Abdelrhman Mohamed
Abdelwahab Boualouache

Abdollah Ahmadi

Abdon Atangana

Abdu Gumaei

Abdul Majeed

Abdul Rahim Ferhan

Abdul Rashid Aziz

Abdul Rashid Shariff

Abdulhamit Subasi

Abdullah Aamir Hayat

Abdullah Abuolaim

Abdullah Alharthi

Abdullah Alomari

Abdullah Bayram

Abdullah Lakhan

Abdullah Waqas

Abdulrahman Khalaf Al-Ali

Abdul-Sattar Kaddour

Abebe Belay Adege

Abebe Diro

Abel Cabrera Martínez

Abel Yeboah-Ofori

Abhay Kotnala

Abhijeet Ravankar

Abhijit Ghosh

Abhijit Sarkar

Abhik Banerjee

Abhilash Pandya

Abhimanyu Singh Garhwal

Abhimanyu Thakur

Abhishek Ghosh

Abhishek Kaushik

Abhishek Kumar

Abhishek Kumar Jha 
Abhishek Kundu

Abhishek Sharma

Abhishek Tiwari

Abhishek Venketeswaran

Abhrajit Sengupta

Abid Khan

Abid Nadeem

Abiel Aguilar-González

Abraham Boby Riby

Abu Sadat Md Sayem

Abu Saleh Md Bakibillah

Abu Sayed Chowdhury

Abu Ul Hassan Sarwar Rana

Abubakar Sharafat

Achilleas Samaras

Achim Bittner

Achim Habekost

Achraf Ammar

Ada Fort

Adam Adam Glowacz

Ádám Bárdos

Adam Brysiewicz

Adam Bujnowski

Adam Chromy

Adam Domański

Adam Gąska

Adam Glowacz

Adam Henschke

Adam Horin

Adam Kawalec

Adam Kiersztyn

Adam Klimowicz

Adam Kłodowski

Adam Koliński

Adam Koniuszy

Adam Krzysztof Pilat

Adam L. Kaczmarek

Adam M. Wojciechowski

Adam Maley

Adam Marchewka

Adam Martowicz

Adam Milik

Ádám Nyerges

Adam Reeves

Adam Ringler

Adam Rogowski

Adam Rosiński

Adam Rutkowski

Adam Santorelli

Adam Wisniewski

Adam Wojciechowski
Adam Zagubień

Adam Zając

Adam Ziębiński

Adamantia Stamou

Adel Alti

Adel Fathy

Adela Badau

Adelaida Avino

Adelaide Miranda

Adele Sateriano

Adewumi John Babafemi

Adi S. Abu-Obeidah

Adilson Berveglieri

Adithya Balasubramanyam

Aditya Karnik

Aditya P. Mathur

Admir Mulahusić

Adnan Ahmad Cheema

Adnan Mahmood

Adnan Mujezinović

Adnan Ul Haque

Adnane Noual

Adolfo Ruiz-Calleja

Adolfo Santoro

Adrian Barbu

Adrian Bekasiewicz

Adrian Burlacu

Adrian Elmi-Terander

Adrián Fernández Gavela

Adrian Filipescu

Adrian Gligor

Adrián González-Sieira

Adrian Kapczyński

Adrian Keating

Adrian Kliks

Adrian Korodi

Adrian Martinez-Rivas

Adrian Olaru

Adrian Peidro

Adrian S. Barb

Adrian Sergiu Darabant

Adrian Sterca

Adrian Stern

Adrian Tamayo

Adrian Wilson

Adrian Wlodarczak

Adriana Alexandru

Adriana Dapena

Adriana Ferlazzo

Adriana Peña Pérez Negron

Adriana Puiu 
Adriana Vargas-Nordcbeck

Adrian-Ioan Petrariu

Adrian-Mihail Stoica

Adriano Andrade

Adriano Cola

Adriano Moreira

Adriano Santos

Adriel Araújo

Adrien Ugon

Adrienn Dineva

Adroaldo Raizer

Afaque Manzoor

Afef Fekih

Agapi Mesodiakaki

Agárdi Anita

Agata Gabryelska

Agata Giełczyk

Agata Kocia

Agata Kołakowska

Agata Stanek

Agata Szłapa-Kula

Agathoklis Giaralis

Ageliki Karatza

Aggeliki Sgora

Aggelos Pallikarakis

Agne Paulauskaite-Taraseviciene

Agnelo R. Silva

Agnes Purwidyantri

Ágnes Vathy-Fogarassy

Agnese Sbrollini

Agnieszka Adamczuk

Agnieszka Barczak

Agnieszka Bitkowska

Agnieszka Blokus

Agnieszka Choroszucho

Agnieszka Dołhańczuk-Śródka

Agnieszka Dudziak

Agnieszka Fiszer

Agnieszka Gadomska-Gajadhur

Agnieszka Kącka-Zych

Agnieszka Konys

Agnieszka Królicka

Agnieszka Lazarowska

Agnieszka Łupicka-Słowik

Agnieszka Mlynarska

Agnieszka Pękala

Agnieszka Stolarczyk

Agnieszka Szczęsna

Agnieszka Szmelter-Jarosz

Agnieszka Szypłowska

Agnieszka Tercjak
Agnieszka Tomaszewska

Agnieszka Wareńczak

Agnieszka Więckowska

Agnieszka Wosiak

Agostino Cortesi

Agostino Forestiero

Agostino Iadicicco

Agostino Occhicone

Aguinaldo Fraddosio

Agustin L. Herrera-May

Agustin Zaballos Diego

Agustın-Agüera Pérez

Ahad Behboodi

Ah-Lian Kor

Ahmad Ali

Ahmad Al-Khasawneh

Ahmad Bazzi

Ahmad Farooq

Ahmad Hassan

Ahmad Jahanbakhshi

Ahmad Jalal

Ahmad Jbara

Ahmad Mohsin

Ahmad Mostafa

Ahmad P. Tafti

Ahmad Suliman

Ahmad T. Almutawa

Ahmad Taher Azar

Ahmed A. Ibrahim

Ahmed Abass

Ahmed Abdelaziz

Ahmed Abdelmottaleb Omar

Ahmed Abouhussien

Ahmed Aljaaf

Ahmed Dorrah

Ahmed El Oualkadi

Ahmed El-Awamry

Ahmed Elhattab

Ahmed Elnakib

Ahmed ElSaid

Ahmed ElSayed

Ahmed Ismail

Ahmed Jalal

Ahmed Khaled

Ahmed Mohamed Fahmy Yousef

Ahmed Mohammed

Ahmed Rachid

Ahmed Rady

Ahmed Ramadhan Al-Obaidi

Ahmed Shaharyar Khwaja

Ahmed Zahran 
Ahmed Zainul Abideen

Ahmed Zoha

Ahmet Can Sabuncu

Ahmet Fatih Tabak

Ahmet Zengïn

Ahyoung Lee

Aibin Yan

Ai-Chun Pang

Aida Azadegan

Aiert Amundarain Irizar

Aigars Atvars

Aiguo Song

Ai-hong Ji

Aijun Zhu

Ailan Che

Aileni Raluca Maria

Aimé Lay-Ekuakille

Aimée Mears

Aimin Chang

Aimin Jiang

Aimin Yang

Airat Sakhabutdinov

Airat Zh. Sakhabutdinov

Aishwaryadev Banerjee

Aisling O'Driscoll

Aitor Almeida

Aitor Arriola

Aitor Oyarbide-Zubillaga

Ajay Ashok

Ajay K. Verma

Ajay Kevat

Ajay Pal

Ajay Vikram Singh

Ajeet Kaushik

Ajit Ahlawat

Ajit Jha

Ajith Pattammattel

Ajmal Khan

Akanksha Bhutani

Akbar A. Khatibi

Akhil Kandhari

Akhil Vaid

Akhila Veerubhotla

Akhilesh S. P. Khope

Akhilesh Thyagaturu

Akhtar Hussain

Akihiro Nakatani

Akilu Yunusa-Kaltungo

Akinori Irizawa

Akio Tsuneda

Akira Ikuta
Akitoshi Shiotari

Akkapol Suea-Ngam

Akm Azad Hossain

Akos Kereszturi

Ákos Kukovecz

Ákos Odry

Akrem Sellami

Aku Visuri

Ala Khalifeh

Ala Moradian

Alaa Abdulhady Jaber

Alaa AlZoubi

Alaa Awad Abdellatif

Alaelddin Fuad Yousif Mohammed

Alain Geiger

Alain Tremeau

Alan Calheiros

Alan Davoust

Alan Demetrius Baria Valejo

Alan J. Michaels

Alan McGibney

Alan Meier

Alan T. Litchfield

Alanoud Subahi

Alban Goupil

Alban Kuriqi

Albane Saintenoy

Albena Antonova

Alberico Sonnessa

Albert Argilaga

Albert De Vries

Albert Lee

Albert Modi

Albert Rogers

Albert Sabban

Albert Solernou

Albert Vette

Alberto Barontini

Alberto Calatroni

Alberto Cardoso

Alberto Comesaña-Campos

Alberto Cordoba Izaguirre

Alberto Corigliano

Alberto Doria

Alberto Eloy García Gutiérrez

Alberto Fernandez

Alberto Fernández-Isabel

Alberto Ferrante

Alberto Gallegos

Alberto Gómez-Caballero

Alberto Gregori 
Alberto Izquierdo-Fuente

Alberto Jorge Rosales Silva

Alberto López-Martínez

Alberto Luviano-Juárez

Alberto Martini

Alberto Miguel Bonastre Pina

Alberto Muscio

Alberto Ochoa

Alberto Ochoa-Zezzatti

Alberto Olmo

Alberto Ortiz

Alberto Pedrouzo-Ulloa

Alberto Pellegrinelli

Alberto Reatti

Alberto Reyna Maldonado

Alberto Rico-Yuste

Alberto Rodrigue Martinez

Alberto Rodríguez-Archilla

Alberto Roncaglia

Alberto Sánchez-Alzola

Alberto Tellaeche Iglesias

Alberto Vallan

Alberto Villalonga

Alcenísio J. Jesus-Silva

Aldo Ghisi

Aldo Gordillo

Aldo Jesorka

Aldo Minardo

Aldo Quattrone

Aldobenedetto Zotti

Aleixandre Manuel

Alejandro Alvarado-Lassman

Alejandro Bustos

Alejandro Carrasco

Alejandro Castañeda-Miranda

Alejandro Castillo Atoche

Alejandro Criado Fernández

Alejandro Diaz

Alejandro García-Miranda Ferrari

Alejandro Gómez-Alanís

Alejandro Javaloyes

Alejandro Martinez-Rios

Alejandro Molina Zarca

Alejandro Ramirez-Rojas

Alejandro Rodriguez

Alejandro Roman Loera

Alejandro Santos Martínez-Sala

Alejandro Vasquez-Rifo

Alejandro Vega

Aleksandar Dimitrijevic

Aleksandar Maksimovic
Aleksandar Mešić

Aleksandar Milosavljević

Aleksandar Obradovic

Aleksandar Vakanski

Aleksandar-Saša Milaković

Aleksander Król

Aleksander Lisowski

Aleksander Mendyk

Aleksander Sešek

Aleksander Sniady

Aleksander V. Marusin

Aleksander Wosniok

Aleksander Zidanšek

Aleksandr Klimov

Aleksandr Leonidovich Kazakov

Aleksandr Oreshonkov

Aleksandr Rakhmangulov

Aleksandr Romanov

Aleksandr Sakhnevych

Aleksandra Kawala-Sterniuk

Aleksandra Krampikowska

Aleksandra Kristo

Aleksandra Królikowska

Aleksandra Radulović

Aleksandra Świerczyńska

Aleksandra Szydłowska

Aleksei Tepljakov

Aleksei Udovichenko

Aleksejs Zacepins

Aleksey Anatolievich Zakharenko

Aleksey Ermoshkin

Aleksey Yurevich Kokhanovskiy

Aleksy Kwilinski

Alena Novak Sedlackova

Aleš Fidler

Aleš Hace

Aleš Janota

Ales Prochazka

Aleš Slíva

Aleš Vysocký

Alessandra Anzolin

Alessandra Biancolillo

Alessandra Borghi

Alessandra Capolupo

Alessandra Filippi

Alessandra Lucchese

Alessandra Rizzardi

Alessandra Scotto di Freca

Alessandra Sorrentino

Alessandra Vitanza

Alessandra Zanut 
Alessandro Aldini

Alessandro Bazzi

Alessandro Burgio

Alessandro Capra

Alessandro Casavola

Alessandro Catania

Alessandro Celestini

Alessandro Ceruti

Alessandro Cicolin

Alessandro Cidronali

Alessandro Cilardo

Alessandro Coclite

Alessandro Cultrera

Alessandro Di Benedetto

Alessandro Di Marco

Alessandro Enrico Cesar Redondi

Alessandro Fanti

Alessandro Fedeli

Alessandro Filippeschi

Alessandro Fraleoni-Morgera

Alessandro Franco

Alessandro Gabrielli

Alessandro Galdelli

Alessandro Giuseppe D'Aloia

Alessandro Greco

Alessandro Labella

Alessandro Maria Selvitella

Alessandro Marroni

Alessandro Masullo

Alessandro Meduri

Alessandro Mengarelli

Alessandro Mingotti

Alessandro Morelli

Alessandro Moro

Alessandro Ortis

Alessandro Paolo Daga

Alessandro Pozzebon

Alessandro Puiatti

Alessandro Rasulo

Alessandro Ridolfi

Alessandro Scano

Alessandro Scordo

Alessandro Severino

Alessandro Sopegno

Alessandro Stefano

Alessandro Terenzi

Alessandro Testa

Alessandro Tonacci

Alessandro Torresani

Alessandro Vizzarri

Alessandro Zampogna
Alessandro Zona

Alessio Aboudan

Alessio Basti

Alessio Brutti

Alessio Buzzin

Alessio Cascardi

Alessio De Angelis

Alessio Fascista

Alessio Martinelli

Alessio Pignalberi

Alessio Ricci

Alessio Rossi

Aletha Gómez

Alex Borges Vieira

Alex Butean

Alex Bystrov

Alex Fan Xu

Alex Fragoso

Alex Fuerbach

Alex J. Yuffa

Alex Mouapi

Alex Sandro Roschildt Pinto

Alex Scott

Alex Weddel

Alexander Aleksandrovich Safonov Alexander Alexandrovich Gromov Alexander Bergmann

Alexander Chizhik

Alexander Deisting

Alexander Fedotov

Alexander Ferworn

Alexander Gillerson

Alexander Hunold

Alexander Hvatov

Alexander Ivannikov

Alexander Kalashnikov

Alexander Kocian

Alexander Kölpin

Alexander M. Samoylov

Alexander Morchenko

Alexander Nesterov-Müller

Alexander Omelyanchik

Alexander Palmanshofer

Alexander Robitzsch

Alexander Rouch

Alexander Ryzhov

Alexander S. Machikhin

Alexander Sboev

Alexander Schaum

Alexander Schulz

Alexander Sutin 
Alexander Sutor

Alexander Tarasov

Alexander Tinius

Alexander V. Mantzaris

Alexander Velmuzhov

Alexander Volyar

Alexander Wöhrer

Alexander Yakunin

Alexander Yu Meigal

Alexandra Bousia

Alexandra Duminil

Alexandra Gemitzi

Alexandra Kämpfer-Homsy

Alexandre Brandão

Alexandre Campeau-Lecours

Alexandre Fieno Da Silva

Alexandre Fonseca Brandão

Alexandre G. Evsukoff

Alexandre Locquet

Alexandre M. P. Botas

Alexandre Meyer

Alexandre Nassif

Alexandre Pereira

Alexandre Robichaud

Alexandre Sava

Alexandre Serres

Alexandre Vervisch-Picois

Alexandros El Sachat

Alexandros Karakostis

Alexandros Pitilakis

Alexandros Stergiou

Alexandru Archip

Alexandru Barsan

Alexandru Ghete

Alexandru Isar

Alexandru Lavric

Alexandru Martian

Alexandru Onea

Alexandru Pîrjan

Alexandru Sorici

Alexandru Takacs

Alexandru Vulpe

Alexandru-Nicolae Tudosie

Alexandu Vulpe

Alexei Dmitriev

Alexei Kamshilin

Alexei Nabok

Alexey Beskopylny

Alexey Buzmakov

Alexey Fomin

Alexey G. Voloboy
Alexey Ivanov

Alexey Kashevnik

Alexey Kureev

Alexey S. Kononikhin

Alexey Shitvov

Alexey Shutov

Alexey V. Povolotskiy

Alexey Vorob'ev

Alexey Wolf

Alexios Mylonas

Alexios P. Douvalis

Alexios Papacharalampopoulos

Alexis Mendez

Alfonso Ariza Quintana

Alfonso Gómez-Espinosa

Alfonso González-Briones

Alfonso Guarino

Alfonso Isidro López Díaz

Alfonso Jose Lopez Rivero

Alfonso Maria Ponsiglione

Alfonso Martínez-Nova

Alfonso Mastropietro

Alfonso Muñoz

Alfonso Padilla-Vivanco

Alfonso Prieto Guerrero

Alfonso Zambon

Alfred O. Ankrah

Alfred Stein

Alfred Teischinger

Alfredo Cigada

Alfredo De Leo

Alfredo Falconieri

Alfredo Gardel

Alfredo Gomes

Alfredo Gonzalez

Alfredo Güemes

Alfredo Hernández

Alfredo J. Pérez

Alfredo Márquez Lucero

Alfredo Moreira Caseiro Rocha

Alfredo Peris

Alfredo Pinedo-Alvarez

Alfredo Rocha

Alfredo Rosado Muñoz

Algazy Zhauyt

Alher Mauricio Hernandez

Ali A. Shukur

Ali Almagbile

Ali Al-Naji

Ali Bakhshinejad

Ali Balador 
Ali Boolani

Ali Caglar Özen

Ali Charkhesht

Ali Chelli

Ali Dehghanfirouzabadi

Ali El Amine

Ali Emre Kaplan

Ali Farmani

Ali H. Husseen Al-Nuaimi

Ali Hassan Sodhro

Ali Hosseininaveh

Ali Imam Sunny

Ali Ismail Awad

Ali Jamoos

Ali Johari

Ali Kafash Hoshiar

Ali Kazemian

Ali Kourani

Ali Lalbakhsh

Ali Mehmandoost Kotlar

Ali Mirzaei

Ali Mohammed

Ali Mousivand

Ali Passian

Ali Riza Ekti

Ali Rohan

Ali Tafarojnoruz

Ali Tavallaei

Ali Zolfagharian

Alia Méndez-Albores

Alice Ahlem Othmani

Alice Buffi

Alice Geminiani

Alice Madonia

Alicia E. Torres-García

Alicia Herrero

Alicia Triviño

Alicja Olejniczak

Alicja Wiora

Alija Pašić

Alim Samat

Alin Dragomir

Alin Grama

Alin Zamfiroiu

Alina Barbulescu

Alina Caddemi

Alina Karabchevsky

Alina Pyka

Alina Roitberg

Alina Vasilescu

Alina Wilkowska
Alina-Cristina Bunea

Alin-Mihai Cailean

Aliona Ivanovna Dreglea

Alireza Ahmadian

Alireza Alamdar

Alireza Babaei

Alireza Borhani

Alireza Entezami

Alireza Haqiqatnejad

Alireza Mohammadi

Alireza Tabatabaeenejad

Alisa Kozitsina

Alisa Rudnitskaya

Alison De Oliveira Moraes

Alison Rodger

Alison Scott

Alistair McEwan

Aliyu Aliyu

Aljoscha Rheinwalt

Alkinoos Athanasiou

Allan L. Schaefer

Allie Anderson

Allison L. Clouthier

Allister Gibbons

Allister Loder

Allouis Christophe

Alma Y. Alanis

Almira Ramanaviciene

Almudena Diaz Zayas

Almudena Rivadeneyra

Almus Kenter

Alon Schclar

Alonso Alonso-Alonso

Alp Karakoç

Alper Aksac

Alphus D Wilson

Al-Sakib Pathan

Alti Adel

Altino M. Sampaio

Álvaro Antón

Alvaro Araujo

Alvaro Araujo Pinto

Álvaro Astasio-Picado

Álvaro Briz-Redón

Álvaro De La Llana Calvo

Alvaro Gutierrez

Álvaro Gutiérrez

Alvaro Hernandez Alonso

Alvaro Joffre Uribe Quevedo

Alvaro Llaria

Álvaro Lozano Murciego 
Alvaro Macias

Álvaro Miguel Carneiro Torrinha

Álvaro Moreno Soto

Álvaro Noriega

Alvaro Parres-Peredo

Alvaro Rodriguez

Alvaro Suarez

Álvaro Suárez

Alvise Benetazzo

Alwin Poulose

Amad Zafar

Amadeo J. Arguelles-Cruz

Amaia Mendez Zorrilla

Amal A. Al-Shargabi

Amalia Beatriz Orúe López

Amalia Floriou-Servou

Amalia Luque

Amalia Luque-Sendra

Aman Behal

Aman Kaur

Amanda C. Mills

Amanda Esquivel

Amani Yousef Owda

Amanpreet Kaur

Amany Sarhan

Amarajothi Dhakshinamoorthy

Amardeep Singh

Ambra Fioravanti

Ambur Ramakrishnan

Amedeo Amoresano

Ameena Al Sumaiti

Amélia Martins Delgado

Amelia Trematerra

Amer Zakaria

Americo Correia

Amin Amini

Amin Anjomshoaa

Amin Gorji

Amin Hosseinian-Far

Amin Komeili

Amin Mobasheri

Amin Shotorbani

Amin Talei

Amin Ullah

Amin Zehtabian

Amina Seferagić

Amir Altaf

Amir Asif

Amir Baghdadi

Amir Benzaoui

Amir Galehdar
Amir Ghiami

Amir Haider Malik

Amir Handelman

Amir Hatamie

Amir Hooshiar

Amir Hosein Oveis

Amir Hossein Behbahani

Amir M. Anvar

Amir Masoud Molaei

Amir Mosavi

Amir Nasrollahi

Amir Rahmati

Amir Reza Ramtin

Amir Shemer

Amirhossein Tehranchi

Amirkianoosh Kiani

Amirreza Aghakhani

Amirreza Khodadadian

Amit Dvir

Amit Kumar Batar

Amit Kumar Sikder

Amitabh Mishra

Amitava Datta

Amiya Nayak

Amjad Iqbal

Amjad Y. Majid

Ammar Ahmed

Ammar Alazab

Ammar Armghan

Ammar Elsheikh

Ammar Muthanna

Amna Qureshi

Amol S. Patwardhan

Amos Ng

Amparo Nuñez-Andrés

Amr Adly

Amrit Abrol

An Cong Tran

Ana Aleksić

Ana B. Ruescas

Ana Colim

Ana Dinora Guzman-Chavez

Ana Djuric

Ana Fernández-Tena

Ana Filipa Sequeira

Ana Gonzalez-Marcos

Ana Isabel Barbosa

Ana Isabel De Andrés Rubio

Ana Isabel Molina

Ana Jiménez Martín

Ana Kuzmanić Skelin 
Ana Madureira

Ana Maria Rocha

Ana Novo

Ana Paula Marques Ramos

Ana Rita Elias Brás

Ana Rovisco

Ana Vázquez Alejos

Anabela Oliveira

Anahita Jablonski-Momeni

Anamaria Bjelopera

Ana-Maria Galan

Anand Chandrasekhar

Anand Koirala

Anand Mishra

Anand Nayyar

Anand Paul

Anand Prakash

Anand Raju

Anand Vazhapilli Sureshbabu

Anandhakumar Sukeri

Anando Sen

Anandram Venkatasubramanian

Anargyros Chatzitofis

Anargyros T. Baklezos

Anastasia Angelaki

Anastasia Lavrenko

Anastasia Vybornova

Anastasiia V. Bakhchina

Anastasija Collen

Anastasija Nikiforova

Anastasios Dimou

Anastasios Doulamis

Anastasios Gotzias

Anastasios Kyritsis

Anastasios L. Kesidis

Anastasiya E. Runnova

Anastassios M. Stamatelos

Anatoli Popov

Anatoly N. Reshetilov

Anatoly Saveliev

Anatoly Skripal

Anca Delia Jurcut

Anca Loredana Udriștoiu

Anca Marginean

Anca Miron

Anca Morar

Anda Belciu

Ander Arriandiaga

Anders Bergman

Anders Kaestner

Anderson A. Felix
Anderson S. L. Gomes

Anderson Wedderhoff Spengler

Anderson Zanardi Freitas

Andon Dimitrov Lazarov

Andon Lazarov

Andoni Beriain

Andras Markus

André Aguiar

André B.M. Souza

André Caceres Carrilho

André Eugênio Lazzaretti

André G. Ferreira

André Hürkamp

André Luís Ferreira De Meireles

André Luís Marques Macato

André Luiz Lins Aquino

Andre Marques-Smith

Andre Mora

André Ottoni

Andre Pasa

André Pimenta Freire

Andre Schneider De Oliveira

André Soares

Andrea Abate

Andrea Ballini

Andrea Ballo

Andrea Bizzego

Andrea Bonci

Andrea Caroppo

Andrea Cataldo

Andrea Cesarini

Andrea Chierici

Andrea Chiuri

Andrea Cioncolini

Andrea Cirillo

Andrea De Martin

Andrea Demeco

Andrea Di Liddo

Andrea Di Schino

Andrea Ehrmann

Andrea Farnham

Andrea Fasano

Andrea Francini

Andrea Fusco

Andrea Galtarossa

Andrea Gilioli

Andrea Giorgetti

Andrea Gonzalez-Montoro

Andrea Lechiancole

Andrea Loddo

Andrea Luttgen 


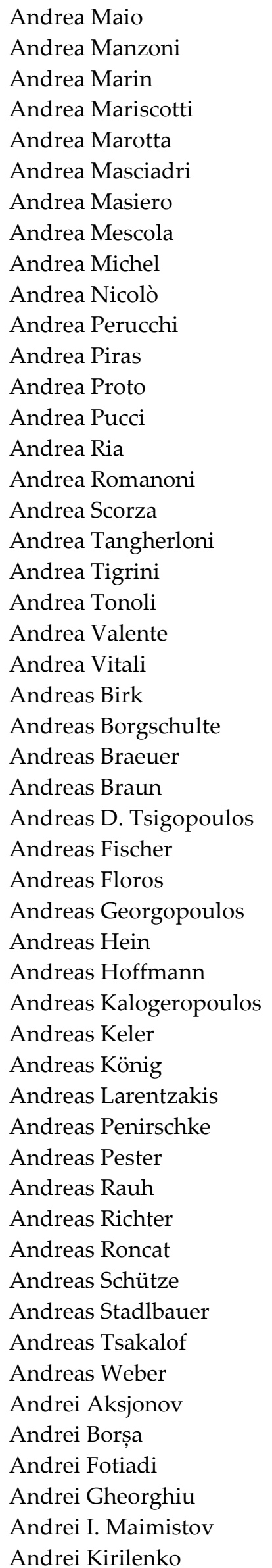

Andrei Lavrinenko

Andrei Olaru

Andrei Potapov

Andrei Rotaru

Andrei Stan

Andrei Stancalie

Andrei Surženkov

Andrei Tarasov

Andrei Tsarev

Andrei Vasilateanu

Andrei Vasile Nastuta

Andrei Vatavu

Andrei Vladyko

Andreia Freitas

Andrej Anžlin

Andrej Bugajev

Andrej Grguric

Andrej Jerman

Andrej Kastrin

Andrej M. Savić

Andrej Novák

Andrej Sarjas

Andrej Zgank

Andreja Trojner-Bregar

Andrés Arcia-Moret

Andres Blanco Ortega

Andrés G. Marrugo

Andrés García Floriano

Andrés J. Prieto

Andrés Omar Tiseira

Andres Öpik

Andres Rubiano

Andrés Sepúlveda Allende

Andrés Trujillo-León

Andrew Aquila

Andrew Bell

Andrew Hamilton

Andrew Hatchett

Andrew Herschfelt

Andrew J. Harvie

Andrew J. Kolarik

Andrew M. Geronimo

Andrew Malone

Andrew Meso

Andrew P Lapointe

Andrew P. Lavender

Andrew Paice

Andrew Peters

Andrew R. Harris

Andrew R. Willis

Andrew Revill 
Andrew Ward

Andrew Wixted

Andrew Wood

Andrey A. Zhirnov

Andrey Afanasiev

Andrey Alekseenko

Andrey Chernov

Andrey D. Pryamikov

Andrey Eliseyev

Andrey Galyaev

Andrey Georgievich Paulish

Andrey I. Poddel'sky

Andrey Koptyug

Andrey Lavrinenko

Andrey Lunkov

Andrey Osipov

Andrey Pryamikov

Andrey Stepnov

Andrey V. Mityakov

Andri Riid

Andrija Bernik

Andrius Čeponis

Andrius Dzedzickis

Andriy Semenov

Andrzej Bęben

Andrzej Bieniek

Andrzej Brudnicki

Andrzej Buchowicz

Andrzej Burghardt

Andrzej Eugeniusz Stateczny

Andrzej Felski

Andrzej Gajewski

Andrzej Gnatowski

Andrzej Janutka

Andrzej Karbowski

Andrzej Kasperski

Andrzej Katunin

Andrzej Kaźmierczak

Andrzej Koszewnik

Andrzej Kotyra

Andrzej Kruk

Andrzej Kubit

Andrzej Kudelski

Andrzej Łebkowski

Andrzej Marcinkowski

Andrzej Materka

Andrzej Michalski

Andrzej Mitura

Andrzej Myśliwiec

Andrzej Nowrot

Andrzej Osuch
Andrzej Pacana

Andrzej Paszkiewicz

Andrzej Pawlowski

Andrzej Perec

Andrzej Polanczyk

Andrzej Przybył

Andrzej Sioma

Andrzej Skoczeń

Andrzej Stateczny

Andrzej Tomporowski

Andrzej Typiak

Andrzej Włoch

Andrzej Zankiewicz

Andy Stamm

Andżelika Krupińska

Anerise De Barros

Aneta Herbut

Aneta Nowak-Michta

Aneta Poniszewska-Maranda

Aneta Prijić

Aneta Ptak-Chmielewska

Aneta Slodek

Anfeng Liu

Angel Carreño-Ortega

Angel Caţaron

Angel Ciprian Cormos

Angel Diéguez

Ángel Giménez Pastor

Angel Jimenez

Ángel Morales-García

Angel Mur

Angel Perez-Cruz

Angel Rodriguez

Ángel Ruiz Zafra

Angela Di Virgilio

Angela Digulescu

Ángela Fernández Pascual

Angela Lombardi

Angela Repanovici

Angélica De Antonio

Angélica Román Guerrero

Angeliki Kritikakou

Angeliki Papalou

Angelo Coluccia

Angelo Galante

Angelo Gulinatti

Angelo Marcelli

Angelo Marcelo Tusset

Angelo Odetti

Angelo Troedhan

Angelos Amditis 
Angelos Karlas

Angelos P. Markopoulos

Angga Hermawan

Anh Thu Phan-Ho

Anh Tran

Anh Truong

Anh-Tu Nguyen

Anhtuan Le

Ania Cravero

Anil B. Shrirao

Anil Kumar Khambampati

Anil Shrirao

Anirban Chowdhury

Anirban Paul

Anirban Sinha

Aniruddha Kaushik

Aniruddha Ray

Anisa Kaur

Anish Prasad Shrestha

Anja Hesselbarth

Anja Keskinarkaus

Anja Schlicht

Anjan Gudigar

Ankana Kakoti

Ankit Jha

Ankit Ravankar

Ankita Sinha

Ann L. Baldwin

Anna A. Okunkova

Anna Banaszek

Anna Barney

Anna Bazan

Anna Bazan-Krzywoszańska

Anna Bergamaschi

Anna Chachaj-Brekiesz

Anna Cleta Croce

Anna D'Auria

Anna Gorbenko

Anna Gubal

Anna Hadamus

Anna Ilnicka

Anna Jamroz-Wisniewska

Anna Kaminska-Chuchmala

Anna Knitter-Piątkowska

Anna Kobusińska

Anna Kruspe

Anna Kuznetsova

Anna M. Rakoczy

Anna Mamou

Anna Michalak

Anna N. Berlina
Anna Pakula

Anna Paradowska-Stolarz

Anna Porębska

Anna Scandurra

Anna Siedliska

Anna Sołtysik-Piorunkiewicz

Anna Staerz

Anna Triantafyllou

Anna Umbert

Anna Vaskuri

Anna Vilà

Anna Visvizi

Anna Zawada-Tomkiewicz

Annalisa D'Arco

Annamaria Costa

Annarita Tedesco

Anne Michelin

Anne Pallarès

Anne Rosken

Anne Schwarz

Annegret Mündermann

Anne-Maria Laukkanen

Annica Kristoffersson

Annika K. Jägerbrand

Anoop Chauhan

Ansgar Schwirtz

Anshu Rastogi

Antal Hiba

Antanas Cenys

Anthony Cahill

Anthony Campbell

Anthony Choi

Anthony Coustou

Anthony Dichiara

Anthony G. Constantinides

Anthony J. Clark

Anthony Neal Watkins

Anthony Pamart

Anthony Waker

Anthoula Gaigneaux

Antoine Dupré

Antoine Fournier

Antoine Schmeitz

Antoine Vacavan

Antoine Weill-Duflos

Antolino Gallego

Antolino Gallego Molina

Anton Antonov

Anton Gradišek

Anton Iliev

Anton Konev 
Anton Konushin

Anton Korniienko

Anton Kos

Anton Manakhov

Anton Pletersek

Anton Popov

Anton Rassõlkin

Anton S. Bychkov

Anton S. Tarasov

Anton Umek

Anton Vershovskii

Antonella Carbonaro

Antonella Curulli

Antonella D'Alessandro

Antonella Falini

Antonella Tatarelli

Antonello Santini

Antoni Grau

Antoni Llopis-Lorente

Antoni Martinez

Antoni Nowakowski

Antoni Perez-Navarro

Antonia Lopreside

Antonija Tadin

Antonin Platil

Antonino Canale

Antonino Laudani

Antonino Masaracchia

Antonino Naro

Antonino Natalello

Antonino Pietropaolo

Antonino Proto

Antonino Quattrocchi

Antonino Quattrone

Antonino S. Fiorillo

Antonino Scandurra

Antonio A. Aguileta

Antonio A. Moya

Antonio Albiol

Antonio Alex Amor

Antonio Balzanella

Antonio Bandera

Antonio Bartolomeo

Antonio Caggiano

Antonio Carlos De Oliveira Júnior

Antonio Carlos Sobieranski

Antonio Cicchella

Antonio Cilfone

Antonio Comparetti

Antonio Corradi

Antonio Cortese
Antonio Costanzo

Antonio Cuccaro

Antonio Di Bartolomeo

Antonio Di Maio

Antonio E. Jimenez-Cano

António Espirito-Santo

Antonio Esposito

Antonio F. Díaz García

Antonio Fernández-Caballero

Antonio Fernandez-Lopez

Antonio Formisano

Antonio García

Antonio Garcia-Jerez

Antonio Gesualdo

Antonio Guillen-Perez

António J. R. Neves

Antonio J. Rodríguez

Antonio J. Sanchez-Salmeron

Antonio J. Torija Martinez

Antonio Jesus Fernandez

Antonio Jesús Yuste Delgado

Antonio José Calderón Godoy

Antonio José Estepa Alonso

Antonio José Lozano Guerrero

Antonio Junior

Antonio Lázaro

Antonio Luiz Pereira De Siqueira Campos

Antonio M. G. Tommaselli

António M. Lopes

Antonio Manzalini

Antonio Matencio Escolar

António Miguel Morgado

Antonio Miguel Ruiz Armenteros

Antonio Minopoli

António Monteiro

Antonio Moschitta

Antonio Muñoz

Antonio Novelli

Antonio Oliveira

Antonio Orlandi

Antonio Pardo

Antonio Parziale

Antonio Pepe

Antonio Pérez-González

Antonio Pescapè

Antonio Petošić

António Pinto

António Ramos

Antonio Ricchi

Antonio Rios-Navarro

Antonio Riul, Jr. 
Antonio Robles-Gómez

Antonio Ruiz Martínez

Antonio Sarasa-Cabezuelo

Antonio Sarolic

Antonio Soriano

António Sousa

Antonio Suppa

Antonio Valente

Antonio Valenzuela Gutiérrez

António Vieira

Antonios Mouratidis

Antonios X. Lalas

Antonis Alexandridis

Antoon Ligtenberg

Antreas Theodosiou

Antti Lehikoinen

Antti Vehkaoja

Anu G. Bourgeois

Anuar De Jesus Fernandez Olvera

Anubhav Srivastava

Anuchit Jitpattanakul

Anuj Tiwari

Anuradha Kar

Anuradha Singh

Anuran Makur

Anuroop Gaddam

Anurup Datta

Anusha Withana

Anusuya Pal

Anxi Yu

An-Yeu Wu

Anyoji Masayuki

Aphrodite Ktena

Apostolos Axenopoulos

Apostolos Gkamas

Apostolos Korlos

Apostolos Kousaridas

Apostolos Papathanassiou

Apostolos Tsagaris

Apostolos Xenakis

Arabas Piotr

Araliya Mosleh

Aram Ter-Sarkisov

Arangarajan Vinayagam

Arantxa Uranga

Arash Ahmadivand

Arash Akbarinia

Arash Jenab

Arash M. Dizqah

Arash Takshi

Arata Suzuki
Arati Sridharan

Aravin Prince Periyasamy

Aravind Sukumaran Rajam

Aravinda Rao

Arbnor Pajaziti

Arcangelo Merla

Ardavan Rahimian

Are Hugo Pripp

Arezoo Emadi

Arfan Ghani

Argyris N. Stassinakis

Argyris Toubekis

Argyro-Maria Boutsi

Ari Hamdani

Arianna Pesci

Arie C. Seijmonsbergen

Ariel Dzwonkowski

Ariel Lellouch

Ariel Soares Teles

Arif Mustafazade

Arif Ul Alam

Arifur R. Khan

Arijit Ghosh

Arinc Ozturk

Arindam Chakrabarty

Arindam Dutta

Aris Psilovikos

Aris Tsangrassoulis

Aris Tsolis

Aristeidis Tsagkaris

Aritz Bilbao-Jayo

Arjun Neupane

Arkadiusz Dobrzycki

Arkadiusz Gardecki

Arkadiusz Kampczyk

Arkadiusz Mystkowski

Arkadiusz Stanula

Arkadiusz Szarek

Arkadiusz Tomczyk

Arkadiusz Zarzycki

Arkady Karyakin

Arkady Redkin

Arkady Serikov

Arlindo Silva

Armando Barreto

Armando Ferreira

Armando J. Pinho

Armando Ricciardi

Armin Lehmann

Armin Masoumian

Armin Mehrabi 
Armin Paravlic

Armin Schmidt

Armir Bujari

Arnab Palit

Arnaldo Batista

Arnaldo Leal-Junior

Arnau Mir Torres

Arnaud Cuisset

Arnaud Gouelle

Arnaud Le Bris

Arnaud Peizerat

Arne Küderle

Arnulfo Ramos-Jiménez

Áron Török

Aroutin Khachaturian

Arpad Gellert

Arpad Karsai

Arsénio Reis

Arslane Hamza-Cherif

Arta Dilo

Artak Heboyan

Artem A. Kuznetsov

Artem Kruglov

Artem Padokhin

Artem Sergeevich Trifonov

Artem V. Nikonorov

Artemios Karvounis

Artemissia-Phoebe Nifli

Arthur Dizon

Arthur I. Petrov

Arthur Molnar

Artjoms Obushevs

Artur Banach

Artur Bejger

Artur Czerwiński

Artur Gafurov

Artur Jorge Ferreira

Artur Kierzkowski

Artur M. Gafurov

Artur Makar

Artur Maurin

Artur Pałasz

Artur Poliński

Artur Rydosz

Artur Spivak

Artur Tadeusz Krzyżak

Artur Wilkowski

Arturas Kaklauskas

Artūras Kilikevičius

Arturas Serackis

Arturo Aparicio Gil
Arturo Garcia-Perez

Arturo Montejo-Ráez

Arturo Reyes-González

Arturo Vera

Artyom Dmitrievich Obukhov

Arumugam Sangili

Arun Arjunan

Arun Jaganathan

Arun Kumar Sangaiah

Arun Kumar Soma

Arun Prakash Periyasamy

Arūnas Lukoševičius

Arunkumar Chandrasekhar

Arup K. George

Arvind Upadhyay

Arxel De León

Asaad Almssad

Asami Kenichi

Asanga Udugama

Asanka G. Perera

Asanthi Jinasena

Asdrúbal López-Chau

Asem M. Ali

Asha Rani

Ashish Goel

Ashish Rauniyar

Ashish Saxena

Ashley Lyons

Ashok Chhetry

Ashok Kumar Patil

Ashok Vaseashta

Ashrafizadeh Seyed Nezameddin

Ashton Theakstone

Ashutosh Bhardwaj

Ashutosh Dhar Dwivedi

Ashutosh Sharma

Ashwin Ashok

Ashwin Kumar Myakalwar

Ashwin Ramesh Babu

Asif Khan

Asim Khan

Asiya Mustafina

Asm Shihavuddin

Asma Adnane

Asma Fallah

Assunta Tavernise

Asta Mikalauskienė

Asterios Leonidis

Atakan Sahin

Atanu Jana

Atefeh Hajijamali Arani 
Atefeh Karimzadeh

Atena Roshan Fekr

Athanasios Anastasiou

Athanasios Dimitriou

Athanasios Koutras

Athanasios Nikolaidis

Athanasios Rakitzis

Athanasios Tiliakos

Athanasios Vasilakos

Athanassios G. Kanatas

Athena Roumboutsos

Athina Alexopoulou

Athina Tsanousa

Athos Trecroci

Atiđa Selmani

Atif Shahzad

Atis Elsts

Atslands Rocha

Atsushi Fukasawa

Atsushi Hosokawa

Atsushi Itou

Atsushi K. Kono

Atsushi Kimura

Atsushi Mitani

Atsushi Motogaito

Atsushi Teramoto

Atta Ur Rehman

Attila Franko

Attila Hilt

Attila Kertesz

Attila Kiss

Attila Nagy

Attila Toth

Atul Kulkarni

Atul Sajjanhar

Audrius Čereška

Audrius Dedele

Audrone Dumciene

Augusto Cesar Da Silva Bezerra

Augusto Ciuffoletti

Augusto Montisci

Augustyn Lorenc

Auk Kim

Auksė Endriulaitienė

Aurel Florentin Cătălin Negrilă

Aurelian Marcu

Aurélie Tournié

Aurélien Arnaubec

Aurélien Dantan

Aurelija Burinskienè

Aurelio Bermúdez
Aurélio Luiz Magalhães Coelho

Aurelio Melo

Aurore Avarguès-Weber

Avanish Mishra

Avgis Hadjipapas

Avi Karsenty

Avinash Sharma

Axel Boese

Axel Buchner

Axel Hahn

Axel Sikora

Ayad M. Fadhil Al-Quraishi

Ayako Nakane

Ayal Anis

Ayan Chatterjee

Ayaz Ahmad

Aydar Nasybullin

Ayham Zaitouny

Aykut Tamer

Ayman El-Zohairy

Aymen Flah

Ayşe Bozlaker

Ayush Dusia

Azhar Abbas

Azhar Zam

Azita Goudarzi

Aziz Amine

Aziz Shah

Azlan Zahid

Azra Tafro

Azwirman Gusrialdi

Azzeddin Naghar

B. N. Pavan Kumar

Babak Jamali

Babak Mohammadi

Babak Shahian Jahromi

Babette Götzendorfer

Babu R. Dawadi

Bac Nguyen

Bader Aldawsari

Badicu Georgian

Bahaa Ansaf

Bahman Javadi

Bahman Moraffah

Bahram Lavi

Bahram Ravani

Bai Li

Bai Xue

Baifan Chen

Bakhtiar Feizizadeh

Bakhtiyar Orazbayev 
Bala Muralikrishnan

Balakrishna Gokaraju

Balázs Heilig

Balázs Kocsi

Balázs Nagy

Balbina Casas-Méndez

Bambang Kuswandi

Bander A. Alzahrani

Baniya Babu

Baodi Liu

Baoding Zhou

Baodong $\mathrm{Xu}$

Baofeng Guo

Baoguo Xu

Baohua Qiang

Baoping Cai

Baoqi Sun

Baoye Song

Bappaditya Mandal

Baptiste Chomette

Barath Raghavan

Barbara Bezerra

Barbara Contiero

Barbara Fabbri

Barbara George-Jaeggli

Barbara Gieroba

Barbara Giussani

Barbara Guidi

Barbara Klein

Barbara Koroušić Seljak

Barbara Leporini

Barbara M. Masini

Barbara S. Chaparro

Barbara Strug

Barbara Vercelli

Barbara Villarini

Barbara Wagner

Bardia Yousefi

Baris Gabriele

Barrie Hayes-Gill

Barry Evans

Barry Lavine

Bart Plovie

Bart Spronck

Bartłomiej Ambrożkiewicz

Bartlomiej Blachowski

Bartłomiej Ćmielewski

Bartłomiej Guzowski

Bartłomiej Szewczyk

Bartłomiej Toroń

Bartolomeo Della Ventura
Bartosz Dalewski

Bartosz Marcinkowski

Bartosz Miller

Bartosz Mindur

Bartosz Mitka

Bartosz Sawik

Bartosz Szeląg

Bartosz Szulczyński

Bartosz Wieczorek

Bas Peters

Basaran Bahadir Kocer

Basil Mohammed Al-Hadithi

Basilio Pueo

Basilio Vescio

Bassel Al Homssi

Bassem Abd-El-Atty

Bastian Engelmann

Bastien Confais

Basuraj Bhowmik

Baudilio Coto García

Bavencoffe Maxime

Bayu Adhi Tama

Bayu Taruna Widjaja Putra

Beat Göpfert

Beata Hejmanowska

Beata Kurc

Beata Łopaciuk-Gonczaryk

Beata Marciniak

Beata Paczosa-Bator

Beata Palczynska

Beáta Stehlíková

Beata Zima

Beatrice Arvinti

Beatrice Motella

Beatriz Garcia-Baños

Beatriz Gomes

Beatriz Gómez-Martín

Beatriz Jurado Sánchez

Beatriz L. Boada

Bee Kang Tan

Beelee Chua

Begoña C. Arrue

Behnam Atazadeh

Behnam Dezfouli

Behrokh Beiranvand

Behrooz H. Yousefi

Behzad Behzad Farahani

Behzad V. Farahani

Behzad Vaferi

Beijia Liu

Beilei Wu 
Beiyu Lin

Béla Genge

Béla Takarics

Belal Ahmad

Belén Curto

Ben Cerjan

Ben Halkon

Ben Johnson

Ben Stansfield

Ben Van Lier

Ben Witvliet

Ben Wolf

Bence Tamás Szabó

Benedetto Barabino

Benedict McLarney

Benedikt Bierer

Benish Fida

Benjamin L. Miller

Benjamin Mentiplay

Benjamin Metcalfe

Benjamin Morrell

Benjamin Turnbull

Bennett I Bertenthal

Bennett Lambert

Bennie Ten Haken

Benoit Charbonnier

Benoit Hilloulin

Benoît Igne

Benoit Parrein

Benoit Picoux

Benoît Piranda

Benoit Piro

Benpeng Zhu

Beomseok Oh

Berend Jan Van Der Zwaag

Beril Sirmaçek

Bernadette Dorizzi

Bernard B. Munyazikwiye

Bernard Kontny

Bernard Pottier

Bernard Schmidt

Bernard Uguen

Bernardo B. Gatto

Bernardo Wagner

Bernd Baumann

Bernd J. Stetter

Bernd-Christian Renner

Bernhard J. Hoenders

Bernhard Koelmel

Bernhard Manhartsgruber

Bernhard Praher
Bernhard Rainer Tittmann

Bernhard Schmauss

Bernhard Zagar

Bert Driessen

Berta Baca-Bocanegra

Berthold Horn

Bertram Taetz

Bertrand David

Bertrand Schneider

Bertrand Simon

Beth Smith

Bethel Osuagwu

Bettina Debû

Bezerianos Anastasios

Bhaben Kalita

Bhagya Samarakoon

Bharanidharan Shanmugam

Bharatesh Chakravarthi

Bharath Babu Nunna

Bharath Ramesh

Bhimsen Rout

Bhogendra Mishra

Biagio Carboni

Biagio Rapone

Bian Tian

Bian $\mathrm{Wu}$

Bianchi Meiguins

Bianchini Carlo

Biao $\mathrm{Hu}$

Biao Kong

Biao Yang

Bifeng $\mathrm{Hu}$

Bih-Chyun Yeh

Bikram Banerjee

Bilal Asad

Bilal H. Alsalam

Bilal Hussain

Bilal Khan

Biliana Georgieva

Bin Chen

Bin Cheng

Bin Gao

Bin Li

Bin Shen

Bin Sheng

Bin Shi

Bin Song

Bin Wang

Bin Wu

Bin Yang

Bin Yin 
Bin Zhang

Binbin Mi

Binbin Yang

Binchun Lu

Bineng Zhong

Bing Li

Bing She

Bing Sun

Bing Wang

Bing Yuan

Bing Zhu

Bingbing Gao

Bingfei Fan

Bingfeng Pan

Bingkun Luo

Bingpu Zhou

Bingqing Luo

Bingrong Miao

Bingrui Chen

Bingwei He

Binh Q. Tran

Binsheng Zhang

Binxin Liu

Biplab Paul

Birgitta Dresp-Langley

Bishnu Regmi

Biswajit Padhy

Biswajit Sarkar

Biswanath Dutta

Bivas Panigrahi

Bjørn Jæger

Björn Krüger

Bjørn Olav Hogstad

Bjørn Skallerud

Blanca Tejedor Herran

Blanka Tundys

Blaž Podgorelec

Blaž Škrlj

Bo Chen

Bo Dai

Bo Fang

Bo Huang

Bo Li

Bo Liu

Bo Mao

Bo Pei

Bo Tan

Bo Wang

Bo Wu

Bo Yang

Bo Yu
Bo Zhang

Bo Zheng

Bobak Mortazavi

Bocai Gao

Bochen Zhang

Bocheng Zhu

Bodor Marius

Bofan Song

Bofeng Guo

Bogdan Cătălin Șerban

Bogdan Constantin Neagu

Bogdan Cristian Florea

Bogdan Dziadak

Bogdan Firtat

Bogdan Florin Filip

Bogdan Gherman

Bogdan Gilev

Bogdan Groza

Bogdan Iancu

Bogdan Lipuš

Bogdan Orza

Bogdan Pankiewicz

Bogdan Răducanu

Bogdan Słodki

Bogdan Socea

Bogdan Strimbu

Bogdan Tiganoaia

Bogdan Zagajewski

Bogdan-Constantin Neagu

Boggarapu Praphulla Chandra

Bogusław Cyganek

Bogusław Szlachetko

Bogusława Kwoczyńska

Bo-Hao Chen

Bohdan Rusyn

Boitumelo Matsoso

Bojan Milovanović

Bojan Stumberger

Bolin Chen

Bo-Lin Jian

Bongsoon Kang

Bon-Gwan Gu

Booma Sowkarthiga Balasubramani

Boonaert Jacques

Bordel Sánchez

Boris Bačić

Boris Dzantiev

Boris G. Vainer

Boris Ginzburg

Boris Igor Palella

Boris Kudryashov 


\begin{tabular}{|c|c|}
\hline Boris Lunin & Brett Hambly \\
\hline Boris Mikhailovich Shumilov & Brian Argrow \\
\hline Boris Miller & Brian Bell \\
\hline Boris N. Fedulov & Brian Bernard \\
\hline Borislav Dimitrov & Brian Birch \\
\hline Borislav Stoyanov & Brian Brown \\
\hline Borivoje Pašić & Brian Butka \\
\hline Borja Bordel Sánchez & Brian Hoover \\
\hline Borja Espejo-García & Brian J. Wisner \\
\hline Borja Genovés Guzmán & Brian N. Kim \\
\hline Borja Peleato & Brian Olshansky \\
\hline Borna Abramović & Brian Rock \\
\hline Bor-Ran Li & Brian Vestal \\
\hline Bor-Shing Lin & Brian Y. Tsui \\
\hline Bor-Shyh Lin & Brice Grunert \\
\hline Bosheng Qin & Brigid Betz-Stablein \\
\hline Bostjan Batagelj & Brik Bouziane \\
\hline Boštjan Kovačić & Briliant Adhi Prabowo \\
\hline Boštjan Markoli & Britt Östlund \\
\hline Boštjan Šumak & Bronisław Stec \\
\hline Botond Sandor Kirei & Brook Galna \\
\hline Boudewijn Van Leeuwen & Bruce Bernacki \\
\hline Bousoulas Panagiotis & Bruce Haycock \\
\hline Bowei Dong & Bruce Stephen \\
\hline Bowen Gong & Bruce T. Tsurutani \\
\hline Bowen Zhu & Bruno Albuquerque De Castro \\
\hline Bowu Zhang & Bruno Andò \\
\hline Boxiang Wang & Bruno Brunone \\
\hline Boxuan Zhong & Bruno Caillier \\
\hline Boyang Li & Bruno De Carvalho Albertini \\
\hline Boyd Fowler & Bruno Ferreira \\
\hline Bozena Borowska & Bruno Fionda \\
\hline Bożena Hoła & Bruno José Nievas Soriano \\
\hline Bożena Jarząbek & Bruno Märkl \\
\hline Bozhao Qi & Bruno Masiero \\
\hline Božidar Potočnik & Bruno O.S. Teixeira \\
\hline Brach Poston & Bruno Silva \\
\hline Brad McDanel & Bruno Sousa \\
\hline Braden M. Li & Bruno Volckaert \\
\hline Bradley Edelman & Bruno Wacogne \\
\hline Brain Via & Bruno Watier \\
\hline Bram Vanderborght & Bugra Alkan \\
\hline Braml Thomas & Bugra Ayan \\
\hline Brandon Foubert & Burak Alakent \\
\hline Branka Stojanovic & Burcu Gumuscu \\
\hline Branko Babusiak & Burkhard Wrenger \\
\hline Branko Kolaric & Bushra Jalil \\
\hline Brayan S. Zapata-Impata & Bushra Zaman \\
\hline Brendan Heery & Buyun Sheng \\
\hline Brett A. Story & Byeong Ha Lee \\
\hline Brett Borghetti & Byeong Hee Kim \\
\hline
\end{tabular}




\begin{tabular}{|c|c|}
\hline Byeong-Choon Goo & Canjun Yang \\
\hline Byeongil Kim & Cao-An Vu \\
\hline Byoung Hun Lee & Capuano Rosamaria \\
\hline ByoungChul Ko & Carina Barbosa Pereira \\
\hline Byoung-Hee Lee & Carina Hahn \\
\hline Bystrík Dolník & Carl James Debono \\
\hline Byung Gwan Hyun & Carl Malings \\
\hline Byung Yong Jeong & Carl Strathearn \\
\hline Byung-Cheol Kim & Carla Queiros \\
\hline Byung-Gyu Kim & Carlo Baldisserri \\
\hline Byung-Gyu Yu & Carlo Bongioanni \\
\hline Byung-hoon Kim & Carlo Castellano \\
\hline Byunghun Lee & Carlo Dossi \\
\hline Byung-Kuk Seo & Carlo Drago \\
\hline Byung-Kwen Song & Carlo Giacomo Leo \\
\hline Byungseok Yoo & Carlo Giglio \\
\hline ByungTae Oh & Carlo Iapige De Gaetani \\
\hline Byungwook Kim & Carlo Massaroni \\
\hline Byung-Wook Park & Carlo Molardi \\
\hline Byungwoon Park & Carlo Morasso \\
\hline C. Ahamed Saleel & Carlo Rainieri \\
\hline Caetano Mazzoni Ranieri & Carlo Requião Da Cunha \\
\hline Cai Liu & Carlo Ricciardi \\
\hline Cai Luo & Carlo Tiebe \\
\hline Cairo L. Nascimento, Jr. & Carlo Tiseo \\
\hline Caisheng Wei & Carlos A. Cifuentes \\
\hline Caitlyn Murphy & Carlos A. P. Soares \\
\hline Caiwei Shen & Carlos Abreu \\
\hline Caleb Rascon & Carlos Alberto Ferreira Marques \\
\hline Calin Ciufudean & Carlos Alex Sander J. Gulo \\
\hline Calin Corciova & Carlos Andres Lara-Nino \\
\hline Călin Vlădeanu & Carlos Andres Luna Vázquez \\
\hline Callum A. Walter & Carlos Capovilla \\
\hline Calvin Eiber & Carlos Carbonell Carrera \\
\hline Camelia Avram & Carlos Carvalho \\
\hline Camelia Cerbu & Carlos Casqueiro \\
\hline Cameron Parvini & Carlos Couder-Castañeda \\
\hline Camila MG Gussen & Carlos D. Garcia-Beltran \\
\hline Camila P. Salomon & Carlos D. Moreno-Moreno \\
\hline Camilla Kärnfelt & Carlos Duarte \\
\hline Camillo Mariani & Carlos E. Galván-Tejada \\
\hline Camillo Ressl & Carlos Eduardo Sanches De Andrade \\
\hline Camilo Díaz & Carlos Eduardo Thomaz \\
\hline Can Huang & Carlos Escobedo \\
\hline Can Koral & Carlos Fernandez-Basso \\
\hline Can Koyuncu & Carlos G. Juan \\
\hline Càndid Reig & Carlos García-Rubio \\
\hline Candido Duarte & Carlos Grilo \\
\hline Canek Portillo & Carlos Guindel \\
\hline Caner Sahin & Carlos Gustavo Resque Dos Santos \\
\hline Canicious Abeynayake & Carlos Gutierrez \\
\hline
\end{tabular}


Carlos Hernando

Carlos Iturrino

Carlos J. Escudero

Carlos Javier Sosa González

Carlos Laranjeira

Carlos Lopez Ardao

Carlos M. Mateo

Carlos M. Travieso-Gonzalez

Carlos Marques

Carlos Marquez

Carlos Medrano

Carlos Moreno-García

Carlos Moron

Carlos Muñoz-Poblete

Carlos Oscar Sanchez Sorzano

Carlos Pascal

Carlos Perez-Ramirez

Carlos Pfeiffer

Carlos Platero

Carlos R. Del-Blanco

Carlos R. Michel

Carlos Renato Menegatti

Carlos Rizo-Maestre

Carlos Roberto Hall Barbosa

Carlos Roberto Minussi

Carlos Roldán-Blay

Carlos Romero Morales

Carlos Rosado

Carlos Senna

Carlos Serodio

Carlos Serrão

Carlos Silva

Carlos Soubervielle-Montalvo

Carlos Torres-Torres

Carlos Travieso-González

Carlos Vaz

Carlos Zafra

Carlos-Alberto Cruz-Villar

Carlos-D. Martínez-Hinarejos

Carme Carrion

Carmelo Corsaro

Carmelo Militello

Carmelo Mineo

Carmelo Sferrazza

Carmen Bisogni

Carmen Cristófol Rodríguez

Carmen Delgado

Carmen Jaren

Carmen Moret-Tatay

Carmen Peláez-Moreno

Carmen Pro
Carmen Voicu

Carmine Ciofi

Carmine Maffei

Carol Corsi

Carol Habib

Carol Maher

Carolina Del-Valle-Soto

Caroline Hartley

Caroline König

Carrillo Dan García

Carson Leung

Carsten Behn

Carsten Dosche

Carsten Laukamp

Casey Chow

Caspar Clark

Casper Boongaling Agaton

Cassio Paiva

Castillo-Rivera Salvador

Cataldo Doria

Cataldo Guaragnella

Catalin Alexandru

Catalin Amza

Cătălin Buiu

Catalin Daniel Caleanu

Catalin I. Pruncu

Catalin Petrescu

Catalin Pruncu

Catalin Stoean

Catalina Rus-Casas

Catalina-Alice Brandus

Catalina-Lucia Cocianu

Catalin-Iosif Ciontea

Catarina Ferreira Da Silva

Catarina Reis

Cate Madill

Caterina Fede

Cathal Heavey

Catherine E. Lang

Catherine Grogan

Cátia Magro

Catia Prandi

Cecilia Clivati

Cecilia Cristea

Cecilia Di Ruberto

Cecilia Jimenez

Cecilia Jimenez-Jorquera

Cecília Sik-Lányi

Cecilia Surace

Cedric Caruana

Cedric Le Gentil 
Celestine Iwendi

Cem Direkoğlu

Cen Tang

Cenek Sasinka

Cengiz Koparan

César Benavente Peces

César Calvo-Lobo

Cesar Camerini

César Cárdenas

Cesar Collazos

Cesar Da Costa

César Elosúa Aguado

César Fernández-Sánchez

César Gattano

César Llamas Bello

César Parcero-Oubiña

César Ricardo Soto-Ocampo

César Yutaka Ofuchi

Cesare Di Girolamo-Neto

Cesare Valenti

Cezary Behrendt

Cezary Kowalczyk

Cezary Orłowski

Cezary Ziółkowski

Chadd W. Clary

Chadin Kulsing

Chadrasekhar Loka

Chae Eun Rhee

Chae-Bong Sohn

Chah Karima

Chaitanya Kumar Suddapalli

Chakchai So-In

Chakfong Cheang

Chan H. See

Chan Hwang See

Chan Su Lee

Chance Tarver

Chandan De

Chandrama Sarker

Chandrasekaran Jayaraman

Chandreswar Mahata

Chang Hong Lin

Chang Kyu Jeong

Chang Li

Chang Liu

Chang Peng

Chang Wang

Chang Won Lee

Chang Wu Yu

Changbao Wen

Changgil Lee
Changho Lee

Changho Yun

Changhong Wang

Changhong Youm

Chang-Hoon Choi

Changhoon Lee

Changhua Zhu

Changjiang Kou

Changqing Cao

Chang-Sei Kim

Chang-Seuk Lee

Changsheng Dai

Chang-Soo Lee

Chang-Wei Hsieh

Changwon Kim

Changyeun Mo

Changyong Cao

Changyong Yim

Changyu Liu

Chang-yue Chiang

Changzhao Liu

Chanjun Jeon

ChanKyu Kang

Chanon Ngamsombat

Chansik Park

Chansu Yang

Chan-Won Park

Chanwoo Moon

Chao Chen

Chao Fu

Chao $\mathrm{Hu}$

Chao Huang

Chao Liu

Chao Lu

Chao Mi

Chao Sun

Chao Tan

Chao Wang

Chao Wu

Chao Xu

Chao Zhai

Chaochen $\mathrm{Gu}$

Chaoching Ho

Chao-Chung Peng

Chaofan Ma

Chaofang Zhao

Chaofeng Wang

Chaofeng Ye

Chao-Hsien Lee

Chaojun Tang

Chaoqun Wang 
Chaoqun Xiang

Chaoran Liu

Chao-Sung Lai

Chaotan Sima

Chao-Tung Yang

Chaouki Hannachi

Chaoxing Liu

Chaoyang Gong

Chaoyang Jiang

Chao-Yang Lee

Chaoyang Ti

Chaoyang Zhang

Chaoyuan Lin

Chaozhe Jiang

Charalambos Sergiou

Charalampos Dimoulas

Charalampos Georgiadis

Charalampos Kalalas

Charalampos Konstantopoulos

Charalampos N. Pitas

Charalampos Orfanidis

Charalampos Valsamos

Charalampos Z. Patrikakis

Charilaos Akasiadis

Charilaos C. Zarakovitis

Charis Ntakolia

Charles Carlson

Charles F. Babbs

Charles Knight

Charles Madewell

Charles Pontonnier

Charles Sackett

Charles Sammut

Charles Sebiyo Batcho

Charlie Catlett

Charlotte Lawson

Chase Shimmin

Chathura Abeywickrama

Chathuranga Kumarage

Chatzichristofis Savvas

Chau Yuen

Chee Kiat Seow

Chee Wei Ang

Chelladurai Karuppiah

Chelsea Dunning

Che-Ming Li

Chen Cao

Chen Chen

Chen Chin-Ling

Chen Shi

Chen Wang
Chen Xing

Chen Yang

Chen Zhao

Chen Zhu

Chenchu Xu

Cheng Chen

Cheng Chi

Cheng $\mathrm{Hu}$

Cheng Siong Chin

Cheng Song

Cheng Tu

Cheng Wu

Cheng $\mathrm{Xu}$

Cheng Yan

Cheng Zong

Chengcai Leng

Cheng-Chi Lee

Cheng-Deng Kuo

Cheng-Hsien Liu

Cheng-Hsiung Hsieh

Cheng-Huan Chen

Cheng-Hung Chuang

Cheng-Hung Lin

Chengjin Qin

Chengkuo Lee

Cheng-Liang Huang

Cheng-Liang Wang

Chenglong Shao

Chengming Sun

Chengqing Li

Chengqing Liu

Cheng-Shiu Chung

Cheng-Te Lin

Cheng-Ting Hsu

Cheng-Wei Fei

Chengwei Zhou

Chengxi Li

Chengxi Zhang

Chengyong Li

Cheng-Yuan Chang

Chengyuan Dong

Chengyuan Liu

Cheng-Zhi Qin

Chenhao Chiu

Chen-Kun Tsung

Chensheng Wu

Chenshuang Li

Chen-Ting Liao

Chen-Wei Chen

Chen-wen Yen

Chenxi Yang 
Chenxing Wang

Chenyu Huang

Cheol Gi Kim

Cheol Song

Cheol-Hong Hwang

Cheol-Hwan You

Cheolsoo Park

Cheon Won Choi

Cheonyong Kim

Chereches Nelu Cristian

Cherlyn J Ng

Chetna Gupta

Che-Wei Lin

Chi Bum Ahn

Chi Cuong Vu

Chi Hou Lei

Chi Lee

Chi Zhang

Chia-Chen Chang

Chia-Chi Cheng

Chia-Chin Chiang

Chiachung Chen

Chia-Hsien Feng

Chia-Hui Liu

Chia-Hung Dylan Tsai

Chia-Min Yang

Chia-Ming Chang

Chiao-Chi Lin

Chiara Allegretti

Chiara Bedon

Chiara Bersani

Chiara Bertolin

Chiara Ceccarini

Chiara Garibotto

Chiara Gruden

Chiara Iacovelli

Chiara Mocenni

Chiara Portesi

Chiara Romei

Chiara Sorbera

Chia-Tai Chan

Chia-Wei Tsai

Chia-Yeh Hsieh

Chi-Ching Chang

Chi-Ching Kuo

Chichung Cheung

Chien-Chang Chen

Chien-Cheng Jung

Chien-Chung Shih

Chien-Hung Li

Chien-Hung Liao
Chien-Nan Kuo

Chien-Sheng Chen

Chien-Sheng Liu

Chien-Tai Hong

Chien-Yao Wang

Chih Chiang Hong

Chih Jer Lin

Chih-Chang Yu

Chih-Chen Yih

Chih-Chieh Liu

Chih-Chieh Yang

Chih-Chin Yang

Chih-Hao Lee

Chih-Hong Lin

Chih-Hsien Huang

Chih-Hsiung Shen

Chih-Hung Wang

Chi-Hieu Pham

Chih-Peng Fan

Chih-Ping Lin

Chih-Sheng Huang

Chi-Hua Chen

Chi-Hung Hwang

Chih-Wei Chiu

Chihwen Hsueh

Chih-Yang Lin

Chih-Yu Kuo

Chih-Yu Wang

Chih-Yu Wen

Chih-Yung Huang

Chi-Jie Lu

Chikahiro Imashiro

Chi-Kuei Wang

Chiman Kwan

Chin-Chi Cheng

Chin-Chun Tsai

Chin-Feng Lin

Ching Biau Tzeng

Ching Torng Lin

Ching-Chi Hsu

Ching-Chou Wu

Ching-Hsin Wang

Ching-Hung Lee

Ching-Jer Huang

Ching-long Shih

Ching-Lung Chang

Ching-Mu Chen

ChingShun Lin

Ching-Ta Lu

Chin-Jen Lin

Chin-Shiuh Shieh 
Chinthaka Premachandra

Chiou-Jye Huang

Chiou-Shann Fuh

Chiranjibi Sitaula

Chiu C. Tan

Chiuan-Chian Chiou

Chiu-Keng Lai

Chiu-kuo Liang

Chi-Wai Chow

Chi-Wen Lung

Chi-Yi Tsai

Chi-Ying Lin

Chi-Yuk Chiu

Cho Nilar Phyo

Chokri Sendi

Chong Hyun Lee

Chong Lei

Chongdeuk Lee

Chongwen Huang

Chongyi Fan

Choongsoo Shin

Choong-un Kim

Chou Ching Lin

Chouikhi Samira

Chouki Sentouh

Chou-Yuan Lee

Chris Dritselis

Chris H. Bahnsen

Chris Hepplewhite

Chris Joslin

Chris McGibbon

Chris Rhodes

Christer Johansson

Christiaan Etienne Vermeulen

Christian A. Cousin

Christian Becker

Christian Bernhard Kuttner

Christian Bräuer-Burchardt

Christian Cabrera Jojoa

Christian Corda

Christian Dobel

Christian Eitzinger

Christian Fernández-Campusano

Christian G. Quintero M.

Christian Galea

Christian Gianoglio

Christian Kehl

Christian Kramberger

Christian Kuttner

Christian Mata Miquel

Christian Mester
Christian Napoli

Christian Peham

Christian Quadri

Christian Rummey

Christian Salim

Christian Santschi

Christian Thom

Christian Vanhille

Christian Viehweger

Christian Vogeley

Christian Werner

Christian-Alexander Bunge

Christiane Thielemann

Christina Kakderi

Christina Keller

Christina Röcke

Christina Salchow-Hömmen

Christina Siontorou

Christina Streli

Christine Detrembleur

Christine Dewi

Christine Gabardo

Christine Kallmayer

Christine Letrou

Christodoulos Keliris

Christoffer R. Heckman

Christoforos Ntantogian

Christoph Bayer

Christoph Bode

Christoph Hartmann

Christoph Hintermüller

Christoph Hoog Antink

Christoph Kralovec

Christoph Rasche

Christoph Reich

Christoph Reichert

Christoph Ritter

Christoph Stach

Christoph Steup

Christoph Tholen

Christoph Wasserzier

Christophe Delebarre

Christophe Jallais

Christophe Kolski

Christophe Lohr

Christophe Rosenberger

Christophe Voisin

Christopher A. G. Kalnins

Christopher Adcock

Christopher Baer

Christopher Ballmann 
Christopher Beach

Christopher Bystroff

Christopher Chesher

Christopher Dicesare

Christopher G Healey

Christopher G. Wilson

Christopher Holmes

Christopher Kent

Christopher Paolini

Christopher Stewart

Christopher T. Goodin

Christos Alexakos

Christos Douligeris

Christos Ioakeimidis

Christos K. Michail

Christos Kokkinos

Christos Koulamas

Christos L. Stergiou

Christos Michail

Christos Mourtzios

Christos Nikolopoulos

Christos P. Antonopoulos

Christos Pappas

Christos Rahiotis

Christos Riziotis

Christos S. Antonopoulos

Christos Spandonidis

Christos Tjortjis

Christos Troussas

Christos Tselios

Christos Verikoukis

Christyan Cruz Ulloa

Chrysafis Andreou

Chu Ma

Chuadhry Mujeeb Ahmed

Chuan Li

Chuangchuang Sun

Chuang-Yuan Chiu

Chuanliang Li

Chuanqi Tan

Chuansi Gao

Chuanxing Bi

Chuanyin Xiong

Chuan-Zhi Dong

Chulhong Kim

Chulhun Kang

Chun Hung Chu

Chun Lin

Chun Pang-jo

Chun Qi

Chun Zhao
Chun-An Cheng

Chunchi Ma

Chunfeng Ma

Chung Horng Lung

Chung Ket Thein

Chung-Chi Huang

Chung-Chiun Liu

Chung-De Chen

Chung-Ho E. Lau

Chung-Hsun Sun

Chung-Liang Chang

Chung-Min Wu

Chung-Ping Chang

Chunguang $\mathrm{Hu}$

Chunho Yeom

Chun-Hsiang (Michael) Chuang

Chun-Hsiang Chuang

Chunhua Dong

Chunhua Su

Chunhua Yang

Chunhui Zhao

Chun-Hung Lin

Chun-Kai Wang

Chunlei Wu

Chunlei Xia

Chun-Ling Lin

Chunlong Fei

Chunming Xia

Chun-Ping Jen

Chunrong Peng

Chunsheng Wu

Chun-Wei Tung

Chunxiao $\mathrm{Hu}$

Chunxiu Liu

Chunye $\mathrm{Xu}$

Chun-Yeon Lin

Chun-Yin Yeh

Chun-Ying Huang

Chunyu Lin

Chun-Yuan Lin

Chuong Ngo

Chuong Nguyen

Chuong V. Nguyen

Chuyi Chen

Chyan-Long Jan

Ciavoi Gabriela

Cicerone Laurentiu Popa

Ciesielski Arkadiusz

Cigdem Turan

Cihan Bayindir

Ciming Zhou 
Cintia Micaela Chamorro Petronacci

Ciprian Comsa

Ciprian Dobre

Ciprian Giurcaneanu

Ciprian Lapusan

Ciprian Lupu

Ciprian Nemes

Ciprian Orhei

Ciprian Pocol

Ciprian Rezus

Ciro Gioia

Cise Midoglu

Claas Falldorf

Claire Goursaud

Claire Walton

Clara Simon De Blas

Claude Chaudet

Claudia Canali

Claudia Conti

Claudia Daffara

Claudia Girjob

Claudia Gonzalez Viejo

Claudia Krull

Claudia Lerma

Cláudia Lopes

Claudia Marzi

Claudia Pacurar

Claudia Sciuto

Claudia Zoppetti

Claudine Irles

Claudine Kraan

Claudinei Rodrigues De Aguiar

Claudio Arancibia-Ibarra

Claudio Bruschini

Claudio Estatico

Claudio M. De Farias

Claudio Marche

Claudio Mirarchi

Claudio Oggeri

Claudio Pellegrini

Claudio Savaglio

Claudio Vasapollo

Claus Milkereit

Claus Pahl

Cleber A. Amorim

Clélia Christina Mello-Silva

Clemens Rumpf

Clement Lork

Clemente Ibarra-Castanedo

Cleonilson Protasio De Souza

Cleopatra Bardaki
Cliff Lissenden

Clint Hansen

Codruta Varodi

Colin Drummond

Collodi Giovanni

Colton Grasmick

Coman Cristian

Concepcion Luján-Álvarez

Cong $\mathrm{Pu}$

Cong Yang

Congcong $\mathrm{Ma}$

Congo Tak Shing Ching

Congzheng Han

Conrad Rizal

Constantin Apetrei

Constantin Ilie

Constantin Nistor

Constantin Orasan

Constantin Paleologu

Constantin Vertan

Constantin Volosencu

Constantine Kotropoulos

Constantine Michailides

Constantino Carlos Reyes-Aldasoro

Constantinos K. Zacharis

Constantinos Patsakis

Constantinos Valagiannopoulos

Cord M. Brundage

Cordos Nicolae

Corina Drapaca

Corina J. Bîrleanu

Corina Nüesch

Corinna Harmening

Corinna Schmitt

Corinne Corbau

Cornel Balint

Cornel Cobianu

Cornel Ioana

Cornelia Măirean

Cornelio Yáñez-Márquez

Corneliu Burileanu

Corneliu Munteanu

Corrado Boragno

Corrado Costa

Corrado Lanera

Corrado Rindone

Cortino Sukotjo

Cory Dixon

Cory M. Smith

Cosimo Gentile

Cosimo Ieracitano 
Cosimo Trono

Coskun Joe Dizmen

Coskun Tekes

Cosmin Ancuti

Costantino Balestra

Costas Chaikalis

Costas Lambrinoudakis

Costas Panagiotakis

Courtney Elaine Stewart

Courtney Roper

Courtney Shell

Creed Jones

Crhistian Raffaelo Baldo

Crispin Mutshinda

Cristhian Duran

Cristian Andriesei

Cristian Axenie

Cristian Barz

Cristian Boldisor

Cristian Chilipirea

Cristian Davalos-Saucedo

Cristian De Santis

Cristian E. Simion

Cristian Fosalau

Cristian Marian Mihaescu

Cristian Mateos

Cristian Moral

Cristian Mușuroi

Cristian Olea

Cristian Pasluosta

Cristian Rotariu

Cristian Simion

Cristian Torres

Cristian Tudor Matea

Cristian Vacacela Gomez

Cristian Vasar

Cristian Vendittozzi

Cristian Viespe

Cristian Zet

Cristiana Tudor

Cristian-Cezar Postelnicu

Cristian-Dragoş Dumitru

Cristiano Cordeiro

Cristiano Costa

Cristiano De Marchis

Cristiano Maria Verrelli

Cristiano Paggetti

Cristiano Palego

Cristiano Premebida

Cristina Anna Maria Lo Iacono

Cristina Baglivo
Cristina Bianca Pop

Cristina Bignardi

Cristina Caramelo Gomes

Cristina Caridade

Cristina Castejon

Cristina Cejudo Bastante

Cristina Coman

Cristina Corti

Cristina De Castro

Cristina Gonzalez-Martin

Cristina Gutierrez-Sanchez

Cristina Maria Ribeiro Caridade

Cristina Mihaela Campian

Cristina Mihaela Nicolescu

Cristina Muresan

Cristina Nuzzi

Cristina Pronello

Cristina Rodriguez-Sanchez

Cristina Stolojescu-Crisan

Cristina Tortia

Cristina-Laura Stolojescu-Crişan

Cristina-Sorina Stangaciu

Cristine Agresta

Cristóbal Barba-González

Cristóbal García Pariente

Cristoforo Marzocca

Csaba Antonya

Csaba Dobo-Nagy

Csaba Szász

Cuauhtémoc Sanchez Ramírez

Cuma Tyszkiewicz

Cun Ji

Cyril Fischer

Cyrille Berger

Cyrille Migniot

Czeslaw Suchocki

D. Hudson Smith

D. Lansing Taylor

D'Andrea Lorenzo

Dabetwar Shweta

Dac-Binh Ha

Dachuan Shi

Dadmehr Rahbari

Daehee Kim

Dae-Hyun Lee

Dae-Ki Kang

Dafna Sussman

Dag Roar Hjelme

Dagmar Pavlu

Dagmara Iwańska

Dahai Xia 


\begin{tabular}{|c|c|}
\hline Daiana Priscila Rodrigues-de-Souza & Dan Song \\
\hline Daifa Wang & Dan $\mathrm{Wu}$ \\
\hline Daifeng Peng & Dan Zeng \\
\hline Daisuke Fujimoto & Dan Zhang \\
\hline Daisuke Matsuura & Dana Badau \\
\hline Daiva Makutėnienè & Dana Petcu \\
\hline Dajun $W u$ & Dana Simian \\
\hline Dakun Lai & Dana Stoian \\
\hline Dalbert Matos Mascarenhas & Dana Vasiljević-Radović \\
\hline Dalei Hao & Dan-Adrian Mocanu \\
\hline Dalia De Santis & Dana-Mihaela Petrosanu \\
\hline Dalia Streimikiene & Dandan Ma \\
\hline Dalibor Bartoněk & Dane Westerdahl \\
\hline Dalibor Ciprian & Danfeng Hong \\
\hline Dalibor Štys & Dang Khoa Nguyen \\
\hline Dalius Jucius & Dangdang Shao \\
\hline Dallan Byrne & Dangirutis Navikas \\
\hline Dalton Pessôa Filho & Dani Tost \\
\hline Dalton Snyder & Daniel (Jian) Sun \\
\hline Daluwathu Mulla Gamage Preethichandra & Daniel A. R. Chaves \\
\hline Dalvan Griebler & Daniel Albuquerque \\
\hline Damian Derlukiewicz & Daniel Amo \\
\hline Damian Grzechca & Daniel Antón \\
\hline Damian Hudziak & Daniel Arvidsson \\
\hline Damian Kordos & Daniel Báscones \\
\hline Damian Krawczykowski & Daniel Basso Ferreira \\
\hline Damian Valles & Daniel Baumgarten \\
\hline Damiano Caruso & Daniel Benedikovic \\
\hline Damiano Perri & Daniel Campos \\
\hline Damianos Chatzievangelou & Daniel Carnieto Tozadore \\
\hline Damien Kinet & Daniel Carruth \\
\hline Damien Leduc & Daniel Chagas Do Nascimento \\
\hline Da-Ming Yang & Daniel Chow \\
\hline Damir Filko & Daniel Condurache \\
\hline Damjan Vlaj & Daniel Costa Ramos \\
\hline Damon Shing-Min Liu & Daniel Cremons \\
\hline Dan Chicea & Daniel Diaconu \\
\hline Dan Cojoc & Daniel Doyle \\
\hline Dan Cristian Popa & Daniel Ficzere \\
\hline Dan Dobrota & Daniel Filipe Albuquerque \\
\hline Dan Feldman & Daniel Fischer \\
\hline Dan Garcia Carrillo & Daniel Flores Tapia \\
\hline Dan Grobnic & Daniel Fodorean \\
\hline Dan Li & Daniel G. Costa \\
\hline Dan Luo & Daniel Gahreman \\
\hline Dan Mircea Suciu & Daniel Gaida \\
\hline Dan Moldovanu & Daniel Gutiérrez Reina \\
\hline Dan Necsulescu & Daniel Hein \\
\hline Dan Neculoiu & Daniel Hernández-Sosa \\
\hline Dan Pescaru & Daniel Himr \\
\hline Dan Selisteanu & Daniel Hoffmann \\
\hline
\end{tabular}


Daniel Homocianu

Daniel Ivan Pineda

Daniel J. Schad

Daniel J. Sousa

Daniel Jáuregui-Vázquez

Daniel Jerez-Mayorga

Daniel Kacik

Daniel Konings

Daniel Kostrzewa

Daniel Leightley

Daniel Lisak

Daniel M. De Leon

Daniel Martinez-Marquez

Daniel Mayer

Daniel McGrath

Daniel Migliozzi

Daniel Mihai Toma

Daniel Molter

Daniel Morinigo-Sotelo

Daniel Octavian Melinte

Daniel Paternain

Daniel Pinardi

Daniel Poenar

Daniel Ramos

Daniel Rodríguez Prado

Daniel Rosner

Daniel Saramak

Daniel Ślęzak

Daniel Sola

Daniel Stich

Daniel Sweeney

Daniel Tonelli

Daniel Ulises Campos-Delgado

Daniel Urbán

Daniela B. Claro

Daniela C. Vaz

Daniela De Bartolo

Daniela De Venuto

Daniela Díaz-Alonso

Daniela Fogli

Daniela Isidori

Daniela Jumanca

Daniela Lupi

Daniela Maffiodo

Daniela Monti

Daniela Pia Rosaria Chieffo

Daniela Pöhn

Daniela Tarnita

Daniela Tesařová

Daniela-Elena Popescu

Daniele Ancora
Daniele Barettin

Daniele Borzelli

Daniele Cafolla

Daniele Cocco

Daniele De Martini

Daniele Di Mitri

Daniele Esposito

Daniele Farnesi

Daniele Ferdani

Daniele Giansanti

Daniele Giordan

Daniele Giusto

Danièle Hauser

Daniele Losanno

Daniele Marinelli

Daniele Martini

Daniele Masarone

Daniele Merli

Daniele Pannone

Daniele Piscitelli

Daniele Sofia

Daniele Spoladore

Daniele Tosi

Daniella Jorge De Moura

Daniil N. Bratashov

Danijela Ristic-Durrant

Danil Ivanov

Danil Pimenov

Danilo Avola

Danilo Comminiello

Danilo Gligoroski

Danilo S. Catelli

Danish Farooq

Daniyal Haider

Danling Wang

Dan-Marius Dobrea

Danny K. Y. Wong

Dansheng Wang

Dante Mújica Vargas

Danuta Umiastowska

Danyang Qin

Danyil Kovalov

Daodao Hu

Daoliang Li

Daolin Ma

Daphne Bazopoulou

Daping He

Darci Odloak

Daria Wotzka

Darija Lemic

Darija Lušić Vukić 
Dario Antonelli

Dario Assante

Dario Ballarini

Dario Cazzola

Dario Sabella

Dario Spiller

Dario Stelitano

Dario Tagliaferri

Dario Vieira

Darius Andriukaitis

Darius Eidukynas

Darius Jegelevičius

Darius Plonis

Darius Popovas

Darius Rudinskas

Dariush Ebrahimi

Dariusz Bismor

Dariusz Buchczik

Dariusz Fydrych

Dariusz Gozdowski

Dariusz Grzybek

Dariusz Horla

Dariusz Kardaś

Dariusz Litwin

Dariusz Makowski

Dariusz Mika

Dariusz Pazderski

Dariusz Puchala

Dariusz Sawicki

Dariusz Świetlik

Dariusz Tomaszewski

Dariusz Tomkiewicz

Dariusz Ulbrich

Dariusz Więcek

Dariusz Wójcik

Darja Topolšek

Darko Huljenić

Darko Stipanicev

Darko Vasic

Darko Vušak

Darryl Bassett

Darya Nemova

Darya Novopashina

Darya Tishkevich

Das Avik Kumar

Dat Ngo

Datong Liu

Datong $\mathrm{Wu}$

Dave Gilmore

David A. Benfield

David A. Constable
David Ahmedt-Aristizabal

David Baeza Moyano

David Barrett

David Berbrayer

David Berry

David Berthebaud

David Blanco

David Blazevic

David Boansi

David Boland

David Brčić

David Brus

David Busch

David C.C. Lam

David Cabaleiro

David Camarena-Martinez

David Charles

David Cheneler

David Ching-Fang Shih

David Chunhu Li

David Clunie

David Dobrocky

David Dominguez

David Dubuc

David Eager

David Escobar-Castillejos

David Estrada

David G. Gobbi

David Garzón Ramos

David Gauthier

David Gómez-Andrés

David Gómez-Ortiz

David Gosztola

David Granados-Lieberman

David Guijo-Rubio

David Guiraud

David Hästbacka

David Hely

David Herrero Pérez

David Huang

David Jaime Cocovi Solberg

David Joon Huang Chuah

David L. Cedeño

David Li

David Lizcano Casas

David Luviano-Cruz

David M. Mackie

David Mancha-Triguero

David Masip

David Melendi

David Mercier 
David Moreno Molina

David Moreno-Hernández

David Murià-Vila

David Naranjo-Hernández

David O'Sullivan

David Pallarés Aldeiturriaga

David Peres Da Rosa

David Perpetuini

David Plets

David Rempel

David Rhiger

David Rodríguez Sanz

David Rogers

David Scaradozzi

David Sedláček

David Stratos

David Thiel

David Thorpe

David Valiente

David Valis

David Van Hamme

David Vetturi

David Viejo-Romero

David Zueco

Davide Astolfi

Davide Bacco

Davide Borroni

Davide Brunelli

Davide C. Critello

Davide Calandra

Davide Comite

Davide Conte

Davide Forcellini

Davide Marsano

Davide Merico

Davide Moroni

Davide Notti

Davide Pagano

Davide Pirrone

Davide Polese

Davide Rocco

Davide Settembre Blundo

Davide Valeriani

Davod Poreh

Davood Karimi

Davor Gracin

Davor Stanko

Davorin Ambruš

Davoud Dastan

Davut Izci

Davy P. Gaillot
Dawei Ge

Dawei Wang

Dawen Zeng

Dawid Janas

Dawid Połap

Dawid Szpak

Dawid Szurgacz

Dawid Wajnert

Dawid Warchoł

Daxin Tian

Dayakar Naik Lavadiya

Dayi Bian

Dayi Zhang

Daylamani-Zad Damon

Dayong Qiao

Dayu Li

Da-Zhi Sun

De Xing Lioe

Debabrata Maity

Debadrita Paria

Debanjana Ghosh

Debankur Sanyal

Debao Yuan

Debbie Rhea

Debiao Lu

Debkalpa Goswami

Débora Christina Muchaluat-Saade

Debora Griffin

Deborah Galpert

Dechao Chen

Declan O'Loughlin

Dedi Setiawan

Deema Alshammary

Deepak Balram

Deepak Kumar Khajuria

Deepak Thirunavukarasu

Deepan Balakrishnan

Deepika Koundal

Defu Lin

Dehai Zhang

Deise Santana Maia

Deisy Chaves

Dejan Dragan

Dejan Drajic

Dejan Nikolic

Dejan Žagar

Dejan Zupan

Dejin Zhang

De-Jiu Chen

Dejun Guo

Deliang Xiang 
Delphine Chadefaux

Demba Diallo

Demetrio Iero

Demetrio Lozano

Demetrios Tsesmelis

Demi Ai

De-Ming Kong

Demóstenes Zegarra Rodríguez

Demosthenes D. Vouyioukas

Deng Wang

Deng Xiao

Dengfeng Chai

Dengfeng Kuang

Dengfeng Peng

Denghui He

Dengpan Ye

Dengyong Wang

Dengzhi Liu

Deni Librado Torres-Roman

Denis Bourgeois

Denis Butusov

Denis Chikurtev

Denis Delisle Rodriguez

Denis Garoli

Denis Gracanin

Denis Guilhot

Denis Hamad

Denis Horvath

Denis N. Sidorov

Denis Sidorov

Denis V. Makarov

Denis Vinnik

Denise Paschoal Soares

Denise Wilson

Denise-Penelope Kontoni

Denise-Penelope N. Kontoni

Deniz Gençağa

Dennis Alveringh

Dennis Edler

Dennis Flanagan

Deodato Tapete

Deog-Su Park

Deokwoo Lee

Depeng Wang

Dequan Zhang

Der-Chyuan Lou

Derek M. Hall

Derrick M. Mott

Derya Birant

Des Watson

Desheng Kong
Deshi Li

Desiderio Cano Porras

Desmond Chow

Detlef Lazik

Deval Mehta

Devanjan Bhattacharya

Devarshi Shah

Devrim Akca

Dewey Taylor

Deyan Lin

Dezhi Han

Dezun Zhao

Dhammika Jayalath

Dhananjay Singh

Dhanasekaran Vikraman

Dhanjai Dhanjai

Dheyaa Jasim Kadhim

Dhiren K. Pradhan

Dhouha El Houssaini

Dhugal Lindsay

Di Dong

Di Jin

Di Peng

Di Sun

Di Wang

Di Wu

Di Yang

Diaa Ahmedien

Diana Berbecaru

Diana Popescu

Dianbiao Dong

Diane Gan

Diane M. Styers

Diane Peters

Diange Yang

Dianyin $\mathrm{Hu}$

Didi She

Dieff L. Vita

Diego Alexander Tibaduiza Burgos

Diego Antolín

Diego Barreto Haddad

Diego Betancourt

Diego Casado-Mansilla

Diego Dall'Alba

Diego Di Francesca

Diego Domínguez

Diego Fernández Iglesias

Diego Gachet Paez

Diego Galvan

Diego García-Gil

Diego Jaén-Carrillo 
Diego M. Jiménez-Bravo

Diego Oliva

Diego Passos

Diego Prieto Herráez

Diego Roberto Colombo Dias

Diego Romano

Diego Sales-lerida

Diego Sušanj

Diego Vergara

Dieter Frense

Dieter Schramm

Dihan Md. Nuruddin Hasan

Dijana Petrovska-Delacrétaz

Dikaia E. Saraga

Dilek Koc-San

Dilpreet Buxi

Dima Cheskis

Dimah Dera

Dimas Irion Alves

Dimitar Nikolov Nikolov

Dimitra K. Toubanaki

Dimitra Tsounidi

Dimitri Belli

Dimitrios A. Exarchos

Dimitrios A. Vrachatis

Dimitrios Bargiotas

Dimitrios Bechtsis

Dimitrios Dechouniotis

Dimitrios Doukas

Dimitrios E. Koulouriotis

Dimitrios Fotiadis

Dimitrios Kateris

Dimitrios Katsantonis

Dimitrios Loukatos

Dimitrios Menychtas

Dimitrios Panagiotidis

Dimitrios Pliatsios

Dimitrios Politikos

Dimitrios Psychas

Dimitrios Pyromalis

Dimitrios Raptis

Dimitrios S. Paraforos

Dimitrios Skarlatos

Dimitrios Stratakis

Dimitrios Tsaopoulos

Dimitrios Tselentis

Dimitrios Vlachopoulos

Dimitrios Vogiatzis

Dimitrios-Lavrentios Prousalis

Dimitris Dechouniotis

Dimitris Giakoumis
Dimitris J. Panagopoulos

Dimitris Kalogeras

Dimitris Kanellopoulos

Dimitris Karampatzakis

Dimitris Mourtzis

Dimitris Spathis

Dimitris Vrakas

Dimka I. Ivanova

Dimos A. Triantis

Dimos Triantis

Dinara Sobola

Dinesh Veeran Ponnuvelu

Ding Wang

Dingde $\mathrm{Xu}$

Dingfu Zhou

Dingkang Wang

Dingtian Yang

Dinh Hoa Nguyen

Dinh-Thuan Do

Dinko Begušić

Dinko Oletic

Dinko Osmankovic

Dino Dobrinić

Diogo Gomes

Diogo Monteiro

Diogo Tecelão

Dionisio Rodriguez

Dipankar Behera

Dipankar Maity

Dipankar Mitra

Dirk Hueske-Kraus

Dirk Söffker

DirkJan Veeger

Divanizia Do Nascimento Souza

Divij Bhatia

Diyan Minkov Dimitrov

Djaffar Ould Abdeslam

Djamal Gacemi

Djedjiga Belfadel

Dmitri E. Kvasov

Dmitrii Tumakov

Dmitriy A. Bukreev

Dmitriy Aleksandrovich Martyushev

Dmitriy Karamov

Dmitriy Kritskiy

Dmitriy Potapenko

Dmitriy V. Sotnikov

Dmitriy Yu. Ozherelkov

Dmitry A. Bykov

Dmitry Bankov

Dmitry Budker 
Dmitry Gavrilov

Dmitry Ilvovsky

Dmitry Karpov

Dmitry Kireev

Dmitry Korzun

Dmitry Murashov

Dmitry Namiot

Dmitry O. Dolmatov

Dmitry Pavlyuk

Dmitry Petrov

Dmitry Roshchupkin

Dmitry Skvortsov

Dmitry Telyshev

Dmitry Vlasov

Dmytro Vasylyev

Do Hyeun Kim

Dobromir Pressyanov

Dobrosława Kasprowicz

Dochshanov M. Alden

Dockhorn Alexander

Doga Bilican

Dohoon Kim

Doina Pisla

Domagoj Tolic

Domen Verber

Domenico Bianchi

Domenico Cannatà

Domenico Ciuonzo

Domenico Di Mauro

Domenico Guida

Domenico M. Doronzo

Domenico Pascarella

Domenico Speranza

Domenico Suriano

Domenico Ursino

Domin Koh

Domingo Marrero

Domingos De Sousa Meneses

Dominic J. Wales

Dominic Kristaly

Dominic O'Connor

Dominic Wales

Dominik Łuczak

Dominik Obrist

Dominik Rybarczyk

Dominik Saul

Dominik Siemon

Dominik Skokandić

Dominique Bégin

Dominique Fourer

Dominique Pagnoux
Dominique Rissolo

Domonkos Varga

Don Chathurika Amarathunga

Donal G. Sinex

Donald Fernandes

Donald Hondongwa

Donald Mueller

Donald R. Reising

Donatella Darsena

Donatella Granata

Donato Conteduca

Donato Hernández Fusilier

Donato Luna Moreno

Donato Romano

Dong Chen

Dong Guan

Dong Hyun Jeong

Dong Kim

Dong Kun Noh

Dong Li

Dong Liang

Dong Meng

Dong Min Kim

Dong Wang

Dong Won Kim

Dong Yang

Dongbao Jia

Dongdong $\mathrm{Mu}$

Don-Gey Liu

Dongfeng He

Dongheon Lee

Donghoon Kim

DongHu Nie

Donghuan Qin

Donghui Xie

Donghyeok An

Donghyun Kim

Dong-il Cho

Dongil Han

Dongjian He

Dong-Joo Kim

Dongkai Yang

Dongkyu Lee

Donglai Zhang

Dongliang Xiao

Dongmei Hao

Dongming Xiao

Dongming Zhang

Dongping Xiao

Dong-Seong Kim

Dongsheng Li 
Dongsheng Zhang

Dong-Shik Kim

Dongshu Wang

Dong-Soo Kwon

Dong-Sop Rhee

Dongwei Ren

Dongwon Jung

Dongxing Cao

Dongyan Wei

Dongyi Chen

Dongyi Wang

Dong-You Choi

Dongyu Zhang

Dongyuan Shi

Doo Heon Song

Doo-Hyun Choi

Doo-Seung Um

Dora Luz Ojeda

Dorian Cojocaru

Dorian J. Burnette

Dorijan Radočaj

Dorin Moldovan

Dorota Dziurka

Dorota Iwaszkiewicz-Grzes

Dorota Lewczuk

Dorota Oszutowska-Mazurek

Dorota Siwicka-Gieroba

Dorota Zawieska

Dörte Solle

Doru Ursutiu

Doru-Laurean Baldean

Dou Zhang

Doug Kurrant

Douglas A. Brooks

Douglas Barbin

Douglas G. Goodin

Douglas O'Shaughnessy

Douglas Rutledge

Dragan Dinulovic

Dragan Indjin

Dragan Pamucar

Dragan Perakovic

Dragan Perakovič

Dragana Bajic

Dragana Perić

Drago Bračun

Drago Strle

Dragoljub Gajic

Dragos Arotăriței

Dragoş Ioan Săcăleanu

Dragos Isvoranu
Dragutin Lisjak

Drew Budner

Drongelen Stefan Van

Dror Malka

Du Hwan Kim

Dua Oezsoylu

Duarte Manuel Azevedo Fernandes

Duc-Duy Ho

Duc-Thinh Pham

Duc-Tien Nguyen

Dugan Um

Dulcinea Avouris

Dumitrescu Catalin

Dumitru Cristian

Dumitru Popescu

Dumitru Toader

Dumitru Vieru

Dung-An Wang

Dunja Srpak

Dunwen Wei

Dunzhu Xia

Duo Li

Duo Sheng

Duo Wai Chi Wong

Duojin Wang

Duong Le

Dusan Gleich

Dušan Kopecký

Dusan Maga

Dušan Šimšík

Dushhyanth Rajaram

Dustin Shipp

Duy Le Thai

Eberhard Lehmann

Ebrahim Ghaderpour

Ebrahim Najafzadeh

Ece Özkan

Ece Uykur

Eda Marchetti

Eddy Krueger

Ede Bodoki

Edelberto Franco Silva

Eden Morales Narváez

Eder Mateus Nunes Gonçalves

Edgar Arribas

Edgar Gutiérrez

Edinéia Aparecida Dos Santos Galvanin

Edison Pignaton De Freitas

Edita Rosana Widasari

Edite Figueiras

Edmanuel Cruz 
Edmundas Kazimieras Zavadskas

Edna Dias Canedo

Edna Possan

Edoardo Fiorillo

Edoardo Rella

Edoardo Rubino

Edouard Ivanjko

Edson Eyji Sano

Edson Jansen Miranda

Édson Luis Bolfe

Edson Mitishita

Eduard Figueras

Eduard Llobet

Eduard Muslimov

Eduard S. Sopin

Eduard Stoica

Eduard Zadobrischi

Eduarda Mendes

Eduardo Artal

Eduardo Barrera

Eduardo Cabal-Yepez

Eduardo Casilari Pérez

Eduardo Cuesta

Eduardo G. Souza

Eduardo Juarez

Eduardo M. G. Rodrigues

Eduardo Palermo

Eduardo Parente Ribeiro

Eduardo Quevedo

Eduardo Scheeren

Eduardo Teixeira

Eduardo Torres

Edward Bormashenko

Edward Hoppe

Edward Jacek Gorzelańczyk

Edward Jones

Edward Kah Wei Tan

Edward Kozłowski

Edward Stanisław Oczeretko

Edwin Ordoukhanian

Edyta Kucharska

Edyta Paczos-Grzeda

Ee Jin Teo

Efraim Winocur

Efrain Mejia-Beltran

Efraín Santiago-Rodríguez

Efstathios Konstantinidis

Efstratios Karantanellis

Efthimios Karymbalis

Efthymios N. Lallas

Eftim Zdravevski
Eftychia Pinakoulaki

Eggo U. Thoden van Velzen

Egidio D'Amato

Egidio Lofrano

Egor Ershov

Ehab Mahmoud Mohamed

Ehsan Khoramshahi

Ehsan Kiani Oshtorjani

Ehsan Nazemi

Eiichi Watanabe

Eiji Yoshihara

Eilif Hjelseth

Eimuntas Paršeliūnas

Eirini Eleni Tsiropoulou

Ekaterina Borisova

Ekaterina Kutafina

Ekaterina R. Gasilova

Ekaterina V. Kunitsyna

Ekim Yurtsever

Ekin Ozer

Eklas Hossain

El Mezyani Touria

Ela Tarakci

Elad Dente

Elbaz Abouelmagd

Elder Alpes De Vasconcelos

Eleazar Aguirre Anaya

Eleftherios Sdoukopoulos

Elena A. Grigorieva

Elena A. Zvereva

Elena Amaricai

Elena Basan

Elena Carlotta Olivetti

Elena Constanta Amăricăi

Elena Damiá

Elena De Cos

Elena Dilonardo

Elena Doynikova

Elena Gubar

Elena Helerea

Elena Hernández-Pereira

Elena Kozlova

Elena Lopez-Aguilera

Elena Lucchi

Elena M. Hernández Pereira

Elena Mora

Elena Morotti

Elena Navarro

Elena Nechita

Elena Niculina Dragoi

Elena Olivetti 
Elena Pivarciova

Elena Polisadova

Elena Potapova

Elena Prudnikova

Elena Roibás-Millán

Elena Savelova

Elena Simona Lohan

Elena Sizikova

Elena Stefana

Elena Tomsik

Elena Torta

Elena V. Emelyanova

Elena Villa

Elena Zaitseva

Eleni G. Symeonaki

Eleni Gianni

Eleni Kokinou

Eleni Koustriava

Eleni Stroulia

Eleni Verykouki

Eleonora Alfinito

Eleonora Macchia

Eli Flaxer

Eli Shlizerman

Elia Gatto

Elia Grueso

Eliana Costa E Silva

Elias Dritsas

Elías Escobar-Gómez

Elias Fakiris

Elias Glytsis

Elias Hamann

Eliete Caldeira

Eligiusz Pawłowski

Elio Romano

Elisa Bassoli

Elisa Cabana Garceran Del Vall

Elisa Castagnola

Elisa Kallioniemi

Elisa Mariarosaria Farella

Elisa Mejía-Mejía

Elisa Panero

Elisa Scalco

Elisabetta Canetta

Elisabetta Martini

Elisabetta Sieni

Elisavet (Ellie) K. Stathopoulou

Elise Shepley

Eliska Sedlackova

Elizabeth Rendon-Morales

Ellen Cesewski
Ellen Williams

Elli Petsa

Elman Shahverdiev

Elmer Alexis Gamboa Peñaloza

Eloisa Dezen Kempter

Eloisa Macedo

Eloy López Meneses

Elsa Garmire

Elsa M. Macías

Élvio Rúbio Gouveia

Elvira Fernandez-Ahumada

Elyse Passmore

Elżbieta Jasińska

Elżbieta Krawczyk-Dembicka

Elżbieta Kubera

Elżbieta Łodyga-Chruścińska

Elżbieta Macioszek

Elżbieta Roszkowska

Emad Samir

Emad Samuel Malki Ebeid

Emanuel Aldea

Emanuel Guariglia

Emanuel Peres

Emanuel Puschita

Emanuela Natale

Emanuele Barca

Emanuele Cardillo

Emanuele Cerruto

Emanuele Frontoni

Emanuele Gabriel Margherita

Emanuele Piuzzi

Emanuele Santi

Emanuele Torti

Emerson Galvani

Emi Yuda

Emil Cazacu

Emil Dumic

Emil Dumić

Emil Engelund Thybring

Emil J. Khatib

Emil N. Sobol

Emil Pricop

Emilia Den Boer

Emilia Mihaylova

Emilia Ogodescu

Emiliano Cè

Emiliano Descrovi

Emiliano Tramontana

Emiliano Zampetti

Emilio Andreozzi

Emilio Martines 
Emilio Matricciani

Emilio Sanjurjo

Emilio Sardini

Emily Hao

Emily Sara Matijevich

Emir Benca

Emma Ozanich

Emmanouel T. Michailidis

Emmanouil Barmpounakis

Emmanouil G. Spanakis

Emmanouil Gagaoudakis

Emmanouil Psomiadis

Emmanuel Agu

Emmanuel Conchon

Emmanuel Karlo Nyarko

Emmanuel Le Clézio

Emmanuel Maravelakis

Emmanuel Marin

Emmanuel Ojo Ademola

Emmanuel Ramasso

Emmanuel Topoglidis

Emmelyn Graham

Emre Salman

En Wang

Enea Parimbelli

Enes Erdin

Engin Ciftyürek

Engin Zeydan

Enji Sun

Ennio Bilancini

Ennio Gambi

Enrica Petrucci

Enrico Babilio

Enrico Calore

Enrico Conca

Enrico Corti

Enrico Denti

Enrico Ferrentino

Enrico G. Caiani

Enrico Maria Staderini

Enrico Meli

Enrico Perri

Enrico Petritoli

Enrico Pisoni

Enrico Ravagli

Enrico Staderini

Enrico Verona

Enrico Vezzetti

Enrique A. Navarro-Camba

Enrique Barón

Enrique Bronchalo
Enrique Chirivella-Perez

Enrique Coronado

Enrique Guerrero

Enrique Hernández-Balaguera

Enrique Hernández-Orallo

Enrique Hortal

Enrique J. Herrera-López

Enrique Melgoza-Vázquez

Enrique Nava-Baro

Enrique Prefasi

Enza Panzardi

Enzo Rizzo

Eoin O'Connell

Epimitheas Georgitzikis

Erasmo Cadenas

Erdenebayar Urtnasan

Erdenebileg Batbaatar

Erez Ribak

Erfan Shojaei Barjuei

Erhan Ekmekcioglu

Eric (Tae H.) Lee

Eric Berkson

Eric C. Honert

Eric Campo

Eric Chappel

Eric De Souza Gil

Eric Fujiwara

Eric Hetland

Eric J. R. Parteli

Eric J. Zhang

Eric Lui

Eric Wade

Eric Webster

Erica Nocerino

Erica Volta

Erick Andres Perez Alday

Erick Reyes Vera

Ericka Janet Rechy-Ramírez

Erik Etien

Erik Heijne

Erik Kajati

Erik Kučera

Erik Linstead

Erik Vavrinský

Erika Brattich

Erika Eskina

Erika Zemková

Erlend Larsen

Ermanno Pietrosemoli

Ernest Czermański

Ernest Tyburski 
Ernesto De La Cruz Sánchez

Ernesto Limiti

Ernesto Moya-Albor

Ernesto Sifuentes

Ernesto Zambrano-Serrano

Ernst Niederleithinger

Erol Gelenbe

Eros Borsato

Eros Montin

Erricos-Chaim Ventouras

Ertugrul Taciroglu

Ervin K. Lenzi

Ervin Kamenar

Erving Ximendes

Erwin Kristen

Erwin Peiner

Erwin Wojtczak

Eryk Wolarz

Esben Søvsø Szocska Hansen

Eshagh Farzaneh Joubaneh

Esma Dervisevic

Esmeidel Leal

Esra Akbas

Essam Rashed

Estate (Tato) Sokhadze

Esteban Inga

Esteban Municio

Esteban Obrero-Gaitán

Esteban Peña Pitarch

Esteban Pino

Esteban Tlelo-Cuautle

Esteban Venialgo

Estefanía Núñez Carmona

Ester D' Accardi

Esther Medrano-Sánchez

Esther Salamí

Estrela Ferreira Cruz

Estrella Rausell

Ethan Bayne

Ethan Fetaya

Etienne Lord

Etienne Losson

Euclides Lourenço Chuma

Eugen Lupu

Eugen Mircea Anitas

Eugen Pfann

Eugene Eremchenko

Eugene Postnikov

Eugenia Fagadar-Cosma

Eugenio Aguglia

Eugenio Cavallo
Eugenio Ivorra

Eugenio Marino Merlo

Eugénio Rodrigues

Eugenio Romanchik Kriuchkova

Eugeniusz Koda

Eugeniusz Rosolowski

Eugin Hyun

Eui Chul Lee

Eulalia Balestrieri

Eulicio De Oliveira Lobo-Junior

Eun Joo Rhee

Eun Seo Choi

Eunho Lee

Eunil Seo

Eunjung Choi

Eunsongyi Lee

Euripidis Glavas

Eusebio De-la-Fuente

Eusebio Eduardo Hernandez

Eusebiu Ilarian Ionete

Eva Álvarez

Eva Bagyinszky

Eva Cernadas

Eva Dokladalova

Eva H. Dulf

Eva Krieghoff-Henning

Eva M. Santos López

Eva Maia

Eva María Cirugeda

Eva Mihalyko-Orban

Eva Villegas Portero

Eva Volná

Evaggelos Kaselouris

Eva-Maria Dölker

Evan O'Keeffe

Evando Araújo

Evangelia Karagianni

Evangelia Kouidi

Evangelia Vouvoudi

Evangelina Lara

Evangelos Hristoforou

Evangelos K. Markakis

Evangelos Keramaris

Evangelos Maltezos

Evangelos Markakis

Evangelos Roussos

Evdokimos Konstantinidis

Evelio Ramirez Miquet

Evelyne Knapp

Everardo Inzunza-González

Everardo Vargas-Rodriguez 
Evgenia Adamopoulou

Evgenia Novikova

Evgenii I. Ponomarev

Evgenij Olegovich Ol'Hovik

Evgeny A. Semenishchev

Evgeny Belyaev

Evgeny Khorov

Evgeny Mirkes

Ewa Gorodkiewicz

Ewa Korzeniewska

Ewa Pawluszewicz

Ewa Popko

Ewa Ropelewska

Ewa Sobolewska

Ewa Waszkiewicz

Ewan Thomas

Ewaryst Tkacz

Ezequiel Coscueta

Ezio Preatoni

Ezzaldeen Edwan

F. Fernando Jurado-Lasso

Fabbrocino Giovanni

Fabian Andres Lara Molina

Fabian Friederich

Fabian Höflinger

Fabian Lorig

Fabian Meder

Fabian Reuter

Fabián Riquelme

Fabian Seguel

Fabiano Ferrari

Fabiao Yu

Fabien Bogard

Fabien Buisseret

Fabien Mieyeville

Fabien Picaud

Fabio Acerbi

Fabio Arena

Fábio Azevedo

Fabio Bisegna

Fabio Botta

Fabio Caraffini

Fabio Cassano

Fabio D'Andreagiovanni

Fabio Garzia

Fabio Henrique Pereira

Fábio Henrique Rojo Baio

Fabio La Foresta

Fabio Leccese

Fabio Luca Bonali

Fabio Maggio
Fabio Manfredini

Fabio Mazzariol Santiciolli

Fábio Mendonça

Fabio Menna

Fabio Mottola

Fabio Narducci

Fabio Pareschi

Fabio Patrone

Fabio Principato

Fabio Radicioni

Fabio Rizzo

Fabio Rossi

Fabio Salice

Fabio Sartori

Fabio Sauli

Fabio Savoldi

Fabio Tango

Fabio Viola

Fabio Zobi

Fabiola Spolaor

Fabrice Retiere

Fabrice Sthal

Fabrizio Balducci

Fabrizio Di Pasquale

Fabrizio Fiori

Fabrizio Gabrielli

Fabrizio Granelli

Fabrizio Marra

Fabrizio Palma

Fabrizio Piergentili

Fabrizio Roccaforte

Fabrizio Stesina

Fadi Muheidat

Fagui Liu

Fahad Manzoor Siddiqui

Fahimeh Mohagheghian

Fahmi Khalifa

Faisal Alfouzan

Faisal Jamil

Faisal Mohd-Yasin

Faizan Mehmood

Fakhroddin Nazari

Falin $\mathrm{Wu}$

Faming Huang

Fan Dang

Fan Gao

Fan Jiang

Fan Li

Fan Wu

Fan Ye

Fang Cheng 
Fang Liu

Fangchen Feng

Fangfang Jiang

Fangfang Zhang

Fangjie Yu

Fanglue Zhang

Fangmin Sun

Fangmin $\mathrm{Xu}$

Fangning $\mathrm{He}$

Fangwen $\mathrm{Yu}$

Fangzhao Zhang

Fangzhou Liu

Fania Palano

Fanli Meng

Fanny Spagnolo

Faouzi Derbel

Fapeng Yu

Fa-Qiang Wang

Faranak Shamsafar

Farbod Khameneifar

Fares Al-Shargie

Fares M'zoughi

Farhad Pourpanah

Farhan Amin

Farhan Chowdhury

Fariba Goodarzian

Farid Orudzhev

Farida El-Dars

Fariza Sabrina

Farkas Andrea

Farkhod Makhmudkhujaev

Farman Ali

Farman Ullah

Farong Gao

Farooq Adnan

Faroq Awin

Farouk Hammami

Farshad Moradi Kashkooli

Faruk Rico

Farzad Fereidouni

Farzad Tashtarian

Farzad Zangeneh-Nejad

Farzan Akbaridoust

Farzin Piltan

Fasheng Wang

Fatemeh Alidoost

Fatemeh Hassanipour

Fatemeh Rezaie

Fatih Akkoyun

Fatima Amer Jid Almahri

Fatima Salahdine
Fatima Zohra Benhalouche

Fatimah Khalaf

Fatos Xhafa

Faucher Cyril

Fausta Fiorillo

Faustino Wahaia

Fausto Ferreira

Fausto Fiorillo

Fausto Orsi Medola

Fausto Pedro García Márquez

Fausto Tucci

Favio-Andrés Pavas-Martínez

Fayez Tarsha Kurdi

Fazal Muhammad

Fazekas Attila

Fazio Roberto

Federica Degno

Federica Maietti

Federica Marcolin

Federica Paolicelli

Federica Piccirilli

Federica Verdini

Federico Becattini

Federico Bolelli

Federico Cugurullo

Federico Dios

Federico Galli

Federico Grasso Toro

Federico Karagulian

Federico Moretti

Federico Moro

Federico Pittino

Federico Roggio

Federico Scurti

Federico Tasca

Fei Ding

Fei Fang

Fei Fei

Fei Hao

Fei $\mathrm{Hu}$

Fei Kang

Fei Li

Fei Liu

Fei Lu

Fei Qiao

Fei Qin

Fei Song

Fei Wang

Fei Xing

Fei Yuan

Feifei Yang 
Feiyan $\mathrm{Hu}$

Feiyun Cui

Feldheiser Aarne

Feldshtein Eugene

Felice Lorusso

Felipe Augusto Pereira de Figueiredo

Felipe Costa

Felipe Da Rocha Henriques

Felipe Gómez-Cuba

Felipe Jiménez

Felipe José Aidar

Felipe Orduña

Felipe Orihuela-Espina

Felipe Toledo

Felipe Trujillo-Romero

Felipe Vannucchi De Camargo

Felisberto Pereira

Felismina T.C. Moreira

Felix Albu

Felix Biermann

Felix Escalona Moncholi

Félix Javier Jiménez-Jiménez

Félix Marcos Tejedor

Felix Scholkmann

Felix Weihs

Femi J. Olorunniji

Fen Lin

Feng Chen

Feng Chi Hsu

Feng Ding

Feng Gan

Feng Gao

Feng Hong

Feng Ju

Feng Ke

Feng Li

Feng Lin

Feng Ling

Feng Liu

Feng Niu

Feng Qian

Feng Sun

Feng Wei

Feng Xiao

Feng Yuan

Feng Zheng

Fengchao Xiong

Feng-Cheng Chang

Feng-Cheng Lin

Fengchun Tian

Fenggang Sun
Fenghe $\mathrm{Wu}$

Fenghua Li

Fengming Li

Fengqiang Li

Fengqiu $\mathrm{Xu}$

Feng-Tsun Chien

Fengxia Su

Feng-Yih Yu

Fengyu Li

Fengyuan Liu

Fengzhong Qu

Fenu Gianfranco

Ferdinando Di Martino

Ferdous Sohel

Fereidoun H. Panahi

Ferenc Szodrai

Fereydoun Aghazadeh

Fermi Guerrero-Castellanos

Fermín Bañón

Fernanda Ferreira

Fernanda Rodrigues

Fernando Almeida

Fernando Auat-Cheein

Fernando Boavida

Fernando Castañ Romero

Fernando Castaño

Fernando Chouza

Fernando De La Prieta Pintado

Fernando De Souza Campos

Fernando Ely

Fernando Feijoo

Fernando Ferreira

Fernando Garramiola

Fernando Henrique Magalhães

Fernando L. Teixeira

Fernando M. Araujo-Moreira

Fernando Manuel Lourenço Martins

Fernando Quesada-Pereira

Fernando Ramos Martins

Fernando Rangel De Sousa

Fernando Reinaldo Ribeiro

Fernando Sánchez Lasheras

Fernando Vaquerizo-Villar

Fernando Vidal-Verdú

Ferran Martin

Ferreira De Lucena Junior Vicente

Fidel Núñez-Ramírez

Filip Holik

Filip Maksimovic

Filip Šuligoj

Filipa João 
Filipa Seabra

Filipa Sousa

Filipe Alves

Filipe Arroyo Cardoso

Filipe Barata

Filipe Manuel Clemente

Filipe Neves

Filipe Neves Dos Santos

Filipe Portela

Filipe Silva

Filippo Bastianini

Filippo Battaglia

Filippo Bergamasco

Filippo Biondi

Filippo Brighina

Filippo Costa

Filippo D'Ippolito

Filippo Gambella

Filippo Giammaria Praticò

Filippo Mantovani

Filippo Paganelli

Filippo Pisano

Filippo Poltronieri

Filippo Ubertini

Filippos Giannakas

Filippos Vallianatos

Filiz Bektas Balcik

Filiz Yesilkoy

Finbarr Murphy

Fionn Murtagh

Firas Hassan

Firas Kobeissy

Firdous A. Shah

Firooz Salami

Fisseha M. Alemayehu

Fitri Trapsilawati

Flávia Aparecida Graça

Flavia Causa

Flavia Delicato

Flavien Valensi

Flavio Balsamo

Flavio Borfecchia

Flavio Canavero

Flavio Cirillo

Flavio Escribano

Flavio Esposito

Flávio Figueira

Flavio Henrique Vasconcelos

Flavio Lupia

Flavio Piccoli

Flavio Shimizu
Flavio Zabini

Flaviu Mihai Frigura-Iliasa

Flaviu Tabaran

Flor G. Ortíz-Gómez

Florenc Demrozi

Florence Nyssen

Florent Jangal

Florent Poux

Florentina-Daniela Munteanu

Florian B. Pokorny

Florian Ion Tiberiu Petrescu

Florian Kirchschlager

Florian Klämpfl

Florian Kölbl

Florian Lipsmeier

Florian Metzger

Florian Michaud

Florian Pape

Florian Schaff

Florian Sigeneger

Florin Alexa

Florin Covaciu

Florin Doru Hutu

Florin Girbacia

Florin Molnar-Matei

Florin Oniga

Florin Popa

Florin Postolache

Florin Sala

Florin Valentin Rusca

Florina Cojocaru

Florina Teodorescu

Florina Ungureanu

Florin-Constantin Mihai

Focke Ziemssen

Fodor Marietta

Foivos Michelinakis

Fokko Wieringa

Folea Silviu

Fortunato C. Dualibe

Fortunato Pezzimenti

Fosco M. Vesely

Fossataro Carlotta

Fotios Gioulekas

Fotis Mavromatakis

Fow-Sen Choa

Foziyeh Sohrabi

Franc Jager

France Le Bihan

Francesc Estrany

Francesc Sebe 
Francesc Wilhelmi

Francesca Antonucci

Francesca Borghi

Francesca Bugiotti

Francesca Cordella

Francesca Cova

Francesca Dalia Faraci

Francesca Lionetto

Francesca Loia

Francesca Lonetti

Francesca Malvano

Francesca Manni

Francesca Nardello

Francesca Pennati

Francesca R. Bertani

Francesca Rossi

Francesca Russo

Francesca Venneri

Francesca Venturini

Francesco Aletta

Francesco Antonio Archetti

Francesco Banterle

Francesco Beritelli

Francesco Bertocci

Francesco Bianconi

Francesco Branda

Francesco Cafaro

Francesco Cappuzzello

Francesco Carlo Morabito

Francesco Castellani

Francesco Cauteruccio

Francesco Centurelli

Francesco Chiti

Francesco Clementi

Francesco Cosco

Francesco D'Amato

Francesco De Leo

Francesco De Natale

Francesco De Pace

Francesco Ernesto Pontieri

Francesco Facchini

Francesco Faita

Francesco Ferracuti

Francesco Filippini

Francesco Fuso

Francesco Giacinto Lavacca

Francesco Granata

Francesco Guidi

Francesco Guzzi

Francesco Lamonaca

Francesco Lelli
Francesco Longo

Francesco Maita

Francesco Marinello

Francesco Martelli

Francesco P. Chietera

Francesco Paolo Lovergine

Francesco Pascale

Francesco Potortì

Francesco Quochi

Francesco Raffa

Francesco Renna

Francesco Rossella

Francesco Ruffino

Francesco Santoni

Francesco Scardulla

Francesco Stillo

Francesco Vatalaro

Francesco Verde

Francesco Villecco

Francesco Visentin

Francine Krief

Francis Bennet

Francis J Osonga

Francis Kalloor Joseph

Francis M Boland

Francis M. Grover

Francis Mulolani

Francis Wyffels

Francisco Alburquerque-Sendín

Francisco Alexandre A. Souza

Francisco Alonso Sarría

Francisco António Bucho Cercas

Francisco Avila

Francisco Aznar

Francisco Beltran-Carbajal

Francisco Berral

Francisco Bulnes

Francisco Cabrera

Francisco Cercas

Francisco Chinesta

Francisco Contreras

Francisco Cruz

Francisco Cuevas De La Rosa

Francisco Del Cerro Velázquez

Francisco Eugenio Potestad-Ordóñez

Francisco Fernández Zacarías

Francisco Fernández-Navarro

Francisco Gallegos-Funes

Francisco Gámez

Francisco Garcia-Lagos

Francisco Gomez-Donoso 
Francisco González

Francisco Haces-Fernandez

Francisco J. Aparicio-Navarro

Francisco J. Díaz-Otero

Francisco J. Ferrero Martín

Francisco J. González-Castaño

Francisco J. Martinez

Francisco J. Medrano

Francisco J. Navarro

Francisco J. Rodriguez-Pulido

Francisco Javier Cárcel-Carrrasco

Francisco Javier Cardenal

Francisco Javier Gimeno Blanes

Francisco Javier González-Cañete

Francisco Javier Mesas Carrascosa

Francisco Javier Otamendi

Francisco José García-Peñalvo

Francisco Jose Mora-Gimeno

Francisco López-Huerta

Francisco Luna-Perejón

Francisco M. Padilla

Francisco Manuel Arrabal-Campos

Francisco Manuel Melgarejo

Francisco Manuel Melgarejo Meseguer

Francisco Manuel Moreno-Pino

Francisco Maroto-Molina

Francisco Marques

Francisco Martínez-Álvarez

Francisco Medina

Francisco Miguel Ribeiro Proença Brójo

Francisco Molina

Francisco Molina Martel

Francisco Moreno

Francisco Nieto Escámez

Francisco Perez-Pinal

Francisco Pizarro

Francisco Rego

Francisco Ronay López-Estrada

Francisco-Javier Moreno-Muro

Franciszek Seredynski

Franck Badets

Franco Frattolillo

Franco Furgiuele

Franco Meggio

Franco Pavese

Franco Tedeschi

Francois Nicolas

François Ouellette

Francois Varray

Frank Boochs

Frank Deinzer
Frank Liebisch

Frank Melandso

Frank Missell

Frank P. Missell

Frank R. Ihmig

Frank Raischel

Frank Slyne

Frank T. Ferguson

Frank Van Eerdenburg

Frank Witmer

Frank-Michael Matysik

Frank-Peter Schilling

Frantisek Brumercik

František Kumhála

František Lopot

František Pollák

František Uherek

František Zbořil

Franz Konstantin Fuss

Franz Waldner

Freddy A. Lucay

Frédéric Chapelle

Frédéric Cointault

Frédéric Dehais

Frédéric Dumur

Frédéric Gillot

Frederic Marin

Frederic Melin

Frédéric Nicolas Daussin

Frédéric Vanderhaegen

Frederica Viti

Frederick T. Sheldon

Frederico Miguel Santos

Frederik Beuth

Frédérique Seyler

Fredrik Johansson

Fredrik Karlsson

Fredrik Olsson

Freek Ariese

Fridolin Wild

Fridon Shubitidze

Frieder C. Krafft

Friedrich Haag

Frits Van Der Eerden

Frouke Hermens

$\mathrm{Fu} \mathrm{Li}$

Fuchuan Duan

Fuentes-Aguilar Rita

Fufei Pang

Fuhua Chen

Fujian Ma 
Fujiang Lin

Fu-Kang Wang

Fulin Luo

Fumihide Kojima

Fu-Min Chang

Fumin Zhang

Fuming Sun

Fumio Narita

Fumio Yamazaki

Fumiya Iida

Fupeng Wang

Fusang Zhang

Fusheng Zha

Futai Zhang

Fuwen $\mathrm{Hu}$

Fuxin Du

Fuxing Gu

Fuyuan Xiao

Fysarakis Konstantinos

G. G. Md. Nawaz Ali

Gábor Kertész

Gabor Kosa

Gábor Mocsár

Gabor Soos

Gabor Sziebig

Gábor Vértesy

Gabriel A. E S. Ferraz

Gabriel A. Lopez

Gabriel D. Peckham

Gabriel Dias Rodrigues

Gabriel Domínguez-Maldonado

Gabriel Falcao

Gabriel Fedorko

Gabriel Gonzalez-Serna

Gabriel Hancu

Gabriel Lucian Radu

Gabriel Murariu

Gabriel Neagu

Gabriel Nicolae Popa

Gabriel Oltean

Gabriel Ramos-Ortiz

Gabriel Sanchez-Perez

Gabriela Covatariu

Gabriela Ioana Proştean

Gabriela Kuncová

Gabriela Paunescu

Gabriela Rutkowska

Gabriela Statkiewicz-Barabach

Gabriela Ventura Silva

Gabriel-Alexandru Constantin

Gabriele Bertagnoli
Gabriele Buttafuoco

Gabriele Di Blasio

Gabriele Freni

Gabriele Magna

Gabriele Maria Lozito

Gabriele Meoni

Gabriele Nava

Gabriele Papadia

Gabriele S. De Blasio

Gabriele Scalia

Gabriella Balestra

Gabriella Bognár

Gabriella Casalino

Gabriella Gigante

Gabriella Olmo

Gabriella Tamburro

Gaddi Blumrosen

Gaël Kermarrec

Gael Sebald

Gaelle Lissorgues

Gaëtan Rey

Gaetano Bellanca

Gaetano D. Gargiulo

Gaetano De Luca

Gaetano Gargiulo

Gaetano Palumbo

Gaetano Quattrocchi

Gaetano Ranieri

Gaetano Vacca

Gaffar Hossain

Gaia Pavoni

Gaige Wang

Gai-Ge Wang

Gail Schofield

Gail Whitelaw

Gaitan Nicoleta Cristina

Gajanan Ghodake

Gajdoš Kljusurić Jasenka

Gajendra Pratap Singh

Gaku Imamura

Galatanu Catalin Daniel

Galien Grosjean

Galina B. Slepchenko

Galina Bogdanova

Galina V. Kurlyandskaya

Galya Georgieva-Tsaneva

Gamini Ariyawansa

Ganapati Bhat

Ganbayar Batchuluun

Gandhimathi Velusamy

Ganesh Kumar 
Ganesh Kumar Srinivasan

Ganesh R. Naik

Ganesh Saratale

Gang Chen

Gang Cheng

Gang Kou

Gang Li

Gang Mei

Gang Meng

Gang Wei

Gang Xiong

Gang Xu

Gang Yao

Gangil Byun

Gangqiang Zha

$\mathrm{Gao} \mathrm{Hu}$

Gaoang Wang

Gaofeng Quan

Gaoge $\mathrm{Hu}$

Gaoliang Peng

Gaoyang Shan

Garbiñe Olabarría

Gareth Rees

Garry Allison

Gary Atkinson

Gary Hooper

Gary M. Rothenberg

Gary Storey

Gaspare Giovinco

Gaurab Dutta

Gaurav Choudhary

Gaurav Sharma

Gautham Prasad

Gavriil George Arsoniadis

Gbanaibolou Jombo

Ge Han

Ge Jin

Geb W. Thomas

Geetesh K. Mishra

Geetika Aggarwal

Geir Storvik

Gelayol Golcarenarenji

Gelu Onose

Gema Prats Boluda

Gemechu Garuma

Gemine Vivone

Gemma Tinti

Gen Li

Genggeng Liu

Geng-Shi Jeng

Gengyan Zhao
Gennady Evtugyn

Gennady I. Grigoriev

Gennady Zebrev

Gennaro Nicola Bifulco

Gennaro Vessio

Geoff Fink

Georg Christoph Brunauer

Georg Fischer

Georg Rill

Georg Rose

Georg Schildbach

George A. Papakostas

George Adam

George C. Alexandropoulos

George Cunha Cardoso

George D. Manolis

George Dan Mois

George E. Tsekouras

George E. Violettas

George Floros

George Giakos

George Grouios

George K. Varotsos

George Lazaroiu

George Manis

George Margetis

George Melillos

George Miloshev

George N. Reeke

George Nikitas

George Oguntala

George Papakostas

George Ponchak

George Suciu

George Tsaramirsis

Georges Alquié

Georges Kaddoum

Georgia Sakellari

Georgiana Codina

Georgiana Simion

Georgios A. Kyriakou

Georgios Bouloukakis

Georgios Christopoulos

Georgios Ctistis

Georgios D. Panos

Georgios Dimitrakopoulos

Georgios Drakopoulos

Georgios E. Arnaoutakis

Georgios E. Stavroulakis

Georgios Georgakis

Georgios Giarmatzis 
Georgios Hadjidemetriou

Georgios Karalekas

Georgios Katsikis

Georgios Kavallieratos

Georgios Kostopoulos

Georgios Leontidis

Georgios Mylonas

Georgios N. Tsigaridas

Georgios P. Mastrotheodoros

Georgios Priniotakis

Georgios Samakovitis

Georgios Spathoulas

Georgios Tsaramirsis

Georgios Tsigaridas

Georgios Tsoumanis

Georgy Fedorov

Ge-Peng Ji

Gera Meeta

Gerald T. Grant

Géraldine E. Merle

Geraldo Cernicchiaro

Geraldo Pereira Rocha Filho

Gérard Chalhoub

Gerard Zamora

Gerardo Alguacil De La Blanca

Gerardo Aranguren

Gerardo Flores

Gerardo Ulises Diaz-Arango

Gerasim Vladimirovich Krivovichev

Gerasimos Bastas

Gerasimos Pagiatakis

Gerasimos Theotokatos

Gerd Dobmann

Gerd Reis

Gerd Scholl

Gergely Lautner

Gerhard Aernout Somsen

Gerhard Leitner

Gerhard Muche

Gerhard Muller

Gerhard Paar

Gerhard Pölzl

Gerhard Steinbeck

Gerhard Wilhelm Weber

Gerhard Zangerl

Germán Carro Fernandez

Germano M. Veiga

Gert Kootstra

Ghada Khoriba

Ghada Zamzmi

Ghaith Khalil
Ghaleb Hoblos

Ghasem Abdi

Ghasem Sargazi

Ghazal Rouhafzay

Gheorghe N. Popescu

Gheorghe Nagit

Gheorghe Nechifor

Gheorghe Nicusor Pop

Gheorghe Sebestyen

Gheorghe Zaharia

Gheorghe-Daniel Voinea

Gheorghe-Eugen Subtirelu

Ghiseok Kim

Gholam Hossein Roshani

Gholamreza Mohammadpour

Ghufran Ahmed

Ghulam Hussain

Gia Khanh Tran

Giacinto Barresi

Giacomo Cabri

Giacomo Capizzi

Giacomo Cappon

Giacomo Genovese

Giacomo Muntoni

Giacomo Viccione

Giada Magni

Giamberto Casini

Giampaolo Pitruzzello

Giampiero Scortichini

Gian Bartolo Picotto

Gian Giuseppe Soma

Gian Luigi Gragnani

Gian Luigi Liberti

Gian Piero Gibiino

Giancarla Alberti

Giancarlo Orengo

Giancarlo Rufino

Giancarlo Sorrentino

Giancarlo Sperlì

Giandomenico Caruso

Giandomenico Spezzano

Gianfranco Avitabile

Gianfranco Forlani

Gianfranco Gagliardi

Gianfranco Mitacchione

Giang Nguyen

Gianluca Caparra

Gianluca Esposito

Gianluca Gennarelli

Gianluca Giuffrida

Gianluca Palli 
Gianluca Percoco

Gianluca Reali

Gianluca Ruffato

Gianluca Sarri

Gianluca Tornese

Gianluigi De Falco

Gianluigi Tiberi

Gianmarco Dinelli

Gianni Cario

Gianni Cerro

Gianni D'Angelo

Gianni Pasolini

Giannis Giannoulis

Gianvito Scaringi

Giedre Sabaliauskaite

Giedre Streckiene

Giedrius Janusas

Giernacki Wojciech

Gihan Mendis

Gil Gonçalves

Gil Shalev

Gilbert De Mey

Gilbert Lim

Gilberto Anzueto-Sánchez

Gilberto Corso

Gilberto Rivera

Gilbert-Rainer Gillich

Gil-Jin Jang

Gilles Bourbon

Gilles Labonte

Gilles Lemercier

Gilles Parent

Gilles Roy

Gilson A. Giraldi

Gilson Adamczuk Oliveira

Giner Alor-Hernández

Ginés García-Mateos

Ginés Morales Méndez

Gino Dardanelli

Gino Putrino

Gino Sorbello

Gintautas Daunys

Gintautas Dzemyda

Gintautas Tamulevičius

Ginu Rajan

Gioacchino Tempesta

Giordano Teza

Giorgia Agresti

Giorgia Fiori

Giorgia Giovannini

Giorgia Liguori
Giorgia Sollai

Giorgio Biagetti

Giorgio Bonmassar

Giorgio Ficco

Giorgio Fuggetta

Giorgio Pennazza

Giorgio Poletti

Giorgio Sebastiano Mauro

Giorgio Vilardi

Giorgios Mallinis

Giorgos Alevizopoulos

Giosue Caliano

Giovanna Bucci

Giovanna Concu

Giovanna Orsini

Giovanna Tranfo

Giovanni Angiulli

Giovanni Artale

Giovanni Battista Rossi

Giovanni Bertolini

Giovanni Bortolan

Giovanni Boschetti

Giovanni Bottari

Giovanni Breglio

Giovanni Bruno

Giovanni Bucci

Giovanni Cabassi

Giovanni Crupi

Giovanni De Gasperis

Giovanni Di Domenico

Giovanni Diraco

Giovanni Francesco Solitro

Giovanni Fusina

Giovanni Galeoto

Giovanni Gallo

Giovanni Gigante

Giovanni Leone

Giovanni Leucci

Giovanni Luca Masala

Giovanni Magno

Giovanni Manfredi

Giovanni Maria Sardi

Giovanni Matarese

Giovanni Mottola

Giovanni Nardini

Giovanni Palmerini

Giovanni Paragliola

Giovanni Pau

Giovanni Piccinni

Giovanni Pintore

Giovanni Sotgiu 
Giovanni Vitale

Giraudo Alessandro

Girolamo Costanza

Gisela Müller-Plath

Gisela Pujol Vazquez

Giulia Abate

Giulia Buttazzoni

Giulia Caruso

Giulia Cisotto

Giulia Fracastoro

Giulia Liberati

Giulia Panebianco

Giulia Paparella

Giulia Ronchetti

Giulia Sacco

Giulia Selvolini

Giulio Bernardi

Giulio Biondi

Giulio Caldarelli

Giulio Cottone

Giulio D'Emilia

Giulio Rosati

Giulio Vialetto

Giulio Vignoli

Giuseppe Andreoni

Giuseppe Arrabito

Giuseppe Artese

Giuseppe Averta

Giuseppe Boccignone

Giuseppe Bombino

Giuseppe Brunetti

Giuseppe Campobello

Giuseppe Cantarella

Giuseppe Capobianco

Giuseppe Carbone

Giuseppe Castorina

Giuseppe Casula

Giuseppe Cattaneo

Giuseppe Ciaburro

Giuseppe Coratella

Giuseppe Coviello

Giuseppe D'Alessandro

Giuseppe Del Core

Giuseppe Di Caprio

Giuseppe Di Leo

Giuseppe Di Modica

Giuseppe Di Stefano

Giuseppe Domenico Arrabito

Giuseppe Esposito

Giuseppe Ferri

Giuseppe Jurman
Giuseppe Lacidogna

Giuseppe Loprencipe

Giuseppe Maria Luigi

Giuseppe Mazzarella

Giuseppe Messina

Giuseppe Minervini

Giuseppe Modica

Giuseppe Musumeci

Giuseppe Nitti

Giuseppe Palestra

Giuseppe Pucciarelli

Giuseppe Quero

Giuseppe Riccio

Giuseppe Sarnè

Giuseppe Schirripa Spagnolo

Giuseppe Scotti

Giuseppe Spilotro

Giuseppe Tagliavini

Giuseppe Trusso Sfrazzetto

Giuseppe Turini

Giuseppe Varone

Giuseppe Zappalà

Giuseppina Gini

Giuseppina Pappalardo

Giuseppina Prezioso

Giusy Macrina

Glauco Fontgalland

Gleifer Vaz Alves

Glen Cooper

Gloria Cerasela Crisan

Gloria Cosoli

GM Shafiullah

Go Yamako

Go Yamamoto

Gobe Hobona

Goeran Fiedler

Gokarna Sharma

Gokhan Mumcu

Gokulnath Thandavarayan

Golovan Andrey Andreevich

Gómez Aguilar José Francisco

Gon Sup Kim

Goncal Costa

Goncalo Jesus

Gonçalo Marques

Gonçalo Rodrigues

Gonçalo Santinha

Gong Cheng

Gong Zhang

Gongjin Lan

Gongping Huang 
Gongping $\mathrm{Wu}$

Gonzalo Avaria

Gonzalo César Gutiérrez-Tobal

Gonzalo De Miguel-Vela

Goorak Kwon

Gopi Samudrala

Goran B. Markovic

Goran Kos

Goran Krstacic

Goran Kvaščev

Goran Poparić

Goran Turk

Goran Vasiljević

Gorazd Bombek

Gordon Tao

Gorka Vuletić

Gorkem Memisoglu

Gornushkin Igor

Gottfried Lechner

Gottfried Schwarz

Gotzon Aldabaldetreku

Gourab Dutta Banik

Govindasamy Mani

Govindhan Maduraiveeran

Gowtham Sathyanarayanan

Grace Hwang

Graciela Šterpin-Valić

Grava Cristian

Grazia Barchi

Grazia Giuseppina Politano

Graziella Scandurra

Grazina Korvel

Grażyna Kiliańska-Przybyło

Greenhall John

Greg J. Tallents

Greg Vaughan

Grégoire Lefebvre

Gregor Donaj

Gregor Kos

Gregor Schiele

Gregor Strle

Gregorij Kurillo

Gregorio Procissi

Gregoriy Kaplan

Gregory Barshtein

Gregory Burwell

Gregory Doumenis

Gregory J. Grosicki

Gregory Miller

Gregory P. Carman

Gregory Prigozhin
Gregory Provan

Gregory Q. Wallace

Gregory Reis

Gregory Simons

Greice K.B. Da Costa

Greta Faccio

Grigor Angjeliu

Grigor Nika

Grigoras Gheorghe

Grigorios G. Anagnostopoulos

Grigorios Nasios

Grigoris Kaltsas

Grigory Ivanovich Dolgikh

Grippa Taïs

Grover Zurita

Grzegorz Bieszczad

Grzegorz Blakiewicz

Grzegorz Chladek

Grzegorz Dombek

Grzegorz Filo

Grzegorz Fusiek

Grzegorz J. Nalepa

Grzegorz Janczyk

Grzegorz Jasinski

Grzegorz Karoń

Grzegorz Komarzyniec

Grzegorz Kopecki

Grzegorz Kowzan

Grzegorz Królczyk

Grzegorz Lentka

Grzegorz Ligus

Grzegorz Marcin Wójcik

Grzegorz Mazur

Grzegorz Mieczkowski

Grzegorz Nalepa

Grzegorz Pastuszak

Grzegorz Psuj

Grzegorz Redlarski

Grzegorz Sarwas

Grzegorz Skrabalak

Grzegorz Sobota

Grzegorz Stepniak

Grzegorz Świt

Grzegorz Trybek

Grzegorz Trzciński

Grzegorz Wojcik

Grzegorz Wrzesiński

Grzegorz Zimon

Gu Eon Kang

Gu Kang

Guadalupe Esteban Vazquez-Becerra 
Guan Gui

Guan Xu

Guang Yang

Guangbo Hao

Guangcai Feng

Guangfu Wu

Guanghao Sun

Guanghui Wen

Guangli Li

Guanglong Du

Guanglou Zheng

Guangming Dong

Guangming Xie

Guangming Xiong

Guangming Zhang

Guangpen Zhang

Guangrong Shen

Guangxin $\mathrm{He}$

Guangxing Wang

Guangxu Cheng

Guangyan Huang

Guangyu Qiu

Guangyuan (Clark) Li

Guanjun Liu

Guanqiu Qi

Guanyi Ma

Guanyi Wang

Guanyu Ma

Gueesang Lee

Guennadi Saiko

Guglielmo Cola

Gui Liu

Gui Yun Tian

Guido Belforte

Guido Borghi

Guido Ehrmann

Guido Gentili

Guido La Rosa

Guido Link

Guido Maiello

Guido Marseglia

Guido Panzarasa

Guifang Dong

Guifen Wang

Guigen Liu

Guijin Wang

Guilherme B. Lucas

Guilherme DeSouza

Guilherme Lucio Abelha Mota

Guilherme Luiz Moritz

Guillaume Andrieux
Guillaume Dardenne

Guillaume Debaene

Guillaume Ducournau

Guillaume J. Bilodeau

Guillaume Lozenguez

Guillaume Puel

Guillaume Ricciardi

Guillaume Sandou

Guillemette Ribay

Guillermo Álvarez-Narciandi

Guillermo Cámara-Chávez

Guillermo De Arcas

Guillermo Del Campo Jimenez

Guillermo Gallego

Guillermo Garcia-Torales

Guillermo Hernandez-Ramirez

Guillermo Morales Luna

Guillermo Moreno-Alcantar

Guillermo R. Iglesias

Guillermo Rodriguez-Navas

Guillermo Ronquillo Lomelí

Guillermo Rus

Guillermo Salceda-Delgado

Guillermo Valencia-Palomo

Guiping Wu

Guisong Yang

Guiwei Li

Guixiong Liu

Guiyang Xin

Gulam Rabbani

Gun Li

Guna Hewa

Gungun Lin

Gunnar Schuetz

Gunnstein Frøseth

Gunter Notni

Gunther Notni

Günther Retscher

Gunzung Kim

Guo Jiang

Guo Liang Goh

Guo Liu

Guo Yahui

Guoan Yang

Guo-Cheng Han

Guodong Ye

Guofa Li

Guogang Zhang

Guohao Dai

Guohua Wei

Guokun Liu 
Guoliang Lu

Guoliang Shang

Guolin Sun

Guolong Zheng

Guoman Huang

Guomei Zhang

Guoniu Zhu

Guoqi Qian

Guoqiang Li

Guoqing Lin

Guoqing Zhao

Guosheng Xu

Guo-Shiang Lin

Guoshuai Zhao

Guoxiu He

Guoxuan Lian

Guozhao Ji

Gurjit Singh Walia

Gururaj Kudur Jayaprakash

Gustavious Paul Williams

Gustavo Arroyo-Figueroa

Gustavo Chica Pedraza

Gustavo Gil

Gustavo Mesias

Guy Ayissi Eyebe

Guy Burroughes

Guy Marlair

Guy Mélard

Guyue Zhang

Guzel Ziyatdinova

Gwan-Hui Lee

Gyanendra P. Joshi

Gyani Shankar Sharma

Gyeongsik Yang

György Eigner

György Molnár

György Nádasy

Gyu Myoung Lee

Gyu Weon Hwang

Gyula Mester

H. Peter White

Ha Duong Ngo

Ha Manh Do

Ha Sul Kim

Haakon Elizabeth Lindstad

Hab. Izabela Rejer

Habib Rahman

Haci Baykara

Hada Fongha Ieong

Hadi Delavari

Hadi Moein
Hadia Awad

Hady Habib

Haejoon Jung

Haewon Byeon

Hafiz Muhammad Ali

Hafiz Suliman Munawar

Hafiz Tayyab Rauf

Hafsa Korri-Youssoufi

Hai Bin Chen

Hai Dong

Hai Liu

Hai Ning Liang

Haibin $\mathrm{Wu}$

Haibo Feng

Haibo Gong

Haibo Zhou

Haichao Hong

Haider Ali

Haider Al-Khateeb

Haidi Abdullah

Haidi Ibrahim

Haidong Shao

Haidy Elghamrawy

Haifeng Zhang

Haigang Sui

Haihang Ye

Haihua Chen

Haijie Tong

Haijun Peng

Haili Sun

Hailiang Liu

Hailing Xiong

Hailong Huang

Hailong Yin

Haim Abramovich

Haimei Gong

Haiming Jin

Hai-Pang Chiang

Haipeng Dai

Haiping Huang

Hai-Po Chan

Haiqiang Niu

Haitan Xu

Haitao Yu

Haitao Zheng

Haitham Abu Ghazaleh

Haixin Sun

Haixing Liu

Haiyan Guan

Haiyang Gao

Haiyang Mao 
Haiyang Zou

Haiying Ma

Haiyong Luo

Hajime Kamebuchi

Hajin Choi

Ha-Jin $\mathrm{Yu}$

Hak-Seon Kim

Haleh Nazemi

Halil Ersin Söken

Halina Tarasiuk

Hamdi Zurqani

Hamed Habibi

Hamed Malekmohammadi

Hamed Mohammadbagherpoor

Hamed Yazdanpanah

Hamid R. Sayarshad

Hamid Reza Ahmadi

Hamid Reza Karimi

Hamid Reza Marateb

Hamida Hallil

Hamidreza Fahham

Hammam Alshazly

Hamza Alkhatib

Hamza Baniata

Hamza Boukabache

Hamza Shakeel

Han Ding

Han Eol Lee

Han Gao

$\mathrm{Han} \mathrm{Hu}$

Han Lim Lee

Han Ouyang

Han Sang Ik

Han Zhang

Hana Tomášková

Hanako Asai

Hanan Hindy

Hanatsu Nagano

Hanbai Park

Hanbin Ma

Hanbit Jin

Hanfei Mei

Hang Ding

Hang Guo

Hang Nga Mai

Hang Su

Hangil Joe

Hanho Wang

Hanhoon Park

Hanhui Li

Hani J. Kbashi
Hani Ramadhan

Hanif Adeela

Hanjiang Luo

Han-Joon Kim

Hanjung Song

Hank White

Han-Kyu Choi

Hann Woei Ho

Hanna Bandarenka

Hanna Järveläinen

Hannes Schniepp

Hanno Hildmann

Hannu Nieminen

Hans Hartnagel

Hans Martin Sauer

Hans Martin Schwab

Hans Peter Lang

Hans-Joachim Krause

Hans-Peter Bischof

Han-Wei Chang

Hany F. Atlam

Hao Cheng

Hao Chi

Hao Jiang

Hao Li

Hao Lu

$\mathrm{Hao} \mathrm{Qu}$

Hao Tian

Hao Wang

Hao Xiong

Hao Yang

Hao Zhang

Haocai Huang

Haochen Hua

Haochong Huang

Haofei Yu

Haojun Ai

Haoqi Sun

Haoqing Li

Haoran Chi

Haoran Wang

Haoran Wei

Haradhan Kolya

Harald Moser

Harald Rennhofer

Harby Mostafa

Haret Rosu

Hari Palani

Hariharan Lalgudi

Harikumar Rajaguru

Harini Narayanan 
Harish Chander

Harish Devaraj

Harish Garg

Harleen Kaur

Harold I. Zeliger

Haroldo T. Hattori

Haroon Aman

Haroon Mahmood

Haroon Malik

Harri Hakula

Harsha Kalutarage

Harshad Mishra

Harshkumar Patel

Hartmut F. Witte

Hartmut Hinz

Harumi Hotta

Haruo Kobayashi

Hasan Abbas

Hasan Abdulrahman

Hasan Onur Keles

Hasan S. Mir

Hashim Habiballa

Hasim Altan

Hasmat Malik

Hassan Algadi

Hassan Ali

Hassan Chizari

Hassan El Alami

Hassan Elahi

Hassan Haes Alhelou

Hassan Karimi-maleh

Hassan Nosrati

Hassan Rabah

Hassane Hotait

Hayder Al-Hraishawi

Hayun Chung

Hazi Mohammad Azamathulla

He Fang

Hector Becerra

Héctor Cardona-Reyes

Hector D. Menendez

Hector Eduardo Roman

Hector Erives

Héctor Espinós-Morató

Hector García-Miquel

Héctor Manuel Pérez-Meana

Hector Montes

Héctor Moreno-Ramón

Hector Solar

Hee Chan Kim

Hee Lee-Niinioja
Heechul Jung

Hee-Deok Yang

Hee-Jun Kang

Heejun Roh

Heejung Kim

Heena Rathore

Heeryon Cho

HeeSeok Kim

Heesuk Son

Heewon Jung

Heeyoung Lee

Heghes Bogdan

Hei Law

Hei Wong

Heike Ebendorff

Heike Herper

Heilig Balázs

Heinrich Hussmann

Heinrich Ruser

Heinz Pernegger

Hejun $\mathrm{Wu}$

Helbert Eduardo Espitia

Hélder Fonseca

Helen C. Leligou

Helen Harman

Helena Cristina Vasconcelos

Helena Fernandez Varo

Helena Navas

Helena Rifà-Pous

Helena Svobodová Kosnáčová

Hélène De Cannière

Hélène Niculita-Hirzel

Helene Pillet

Helin Yang

Helinando Pequeno De Oliveira

Helko Borsdorf

Helmut Karl Lackner

Helmut Klöcker

Helon Vicente Hultmann Ayala

Hemal Naik

Hemant Pandit

Hemi Qu

Heming Wei

Hemraj M Yadav

Hendrik Jan Van Der Woerd

Hendrix Demers

Heng Wang

Hengcai Zhang

Hengchang Bi

Hengfeng $\mathrm{Gu}$

Heng-Tung Hsu 
Henri Achten

Henriette Heinrich

Henrik Koblauch

Henrik Zsiborács

Henrique E. M. Peres

Henrique Ferreira Da Silva Paulo

Henrique Mamede

Henrique Silva

Henry Griffith

Henryk Krawczyk

Heoncheol Lee

Herbert Enser

Herbert Kimura

Herbert Michael Heise

Herman C. Myburgh

Herman L. Offerhaus

Herman Van Werkhoven

Hermann Gilly

Hermano Velten

Hermes Giberti

Hernan Gonzalez

Hernán Morales-Navarrete

Hernando P. Bacosa

Herng-hua Chang

Herve Aubert

Hesam Babahosseini

Hesham MH Zakaly

Heshan Liu

He-sheg Wang

Hessam Golmohamadi

Heungjoo Shin

Hezhen Lou

Hiam Khoury

Hichem Mrabet

Hideaki Murayama

Hideaki Nozato

Hideaki Uchiyama

Hidekazu Takano

Hideki Hideki Tomita

Hideki Komagata

Hideki Naito

Hiep Duc Nguyen

Higinio Rubio Alonso

Hikaru Aono

Himanshu Agrawal

Himer Avila-George

Hinrich Grothe

Hira Fatima

Hiram Calvo

Hiroaki Hayashi

Hiroaki Kikuchi
Hiroaki Kuze

Hiroaki Morino

Hiroaki Nishikawa

Hiroaki Satoh

Hirofumi Nonaka

Hirokatsu Kataoka

Hirokazu Madokoro

Hiroki Ashiba

Hiroki Ishizuka

Hiroki Mano

Hiromasa Shimizu

Hirosato Monobe

Hiroshi Churei

Hiroshi Dozono

Hiroshi Higashi

Hiroshi Kagemoto

Hiroshi Matsuo

Hiroshi Ozawa

Hiroshi Tanaka

Hiroyoshi Ohtsu

Hiroyuki Okamoto

Hiroyuki Sato

Hisham Arafat

Hla Myo Tun

Ho Won Jang

Ho Won Lee

Hoa Tran-Dang

Hoang Long Nguyen

Hoang Nguyen

Hoang-Long Cao

Hocheon Yoo

Hocine Cherifi

Hodjat Pendar

Hoerner Stefan

Hoi-Shun Lui

Hojong Choi

Hojoong Kim

Ho-Jun Suk

Holger Babinsky

Holger Hill

Holger Maune

Hong Bao

Hong Chi

Hong Ding

Hong Li

Hong Lin

Hong Liu

Hong Men

Hong Pan

Hong Ren Wu

Hong Song 
Hong Tang

Hong Wang

Hong Xie

Hong Zeng

Hong Zhang

Hong Zhao

Hongbin Lin

Hongbin Pu

Hongbin Sun

Hongbin $\mathrm{Yu}$

Hongbing Deng

Hongbing Ji

Hongbo Gao

Hongbo $\mathrm{Gu}$

Hongbo Wang

Hongbo Zhang

Hongchao Fan

Hongchul Sohn

Hongde Qin

Hongen Liao

Hongfeng $\mathrm{Yu}$

Honggui Li

Hong-Hao Gao

Hongjie Jiang

Hongju Mao

Hong-Jun Xiang

Hongkook Kim

Honglang Li

Hongli $\mathrm{Hu}$

Hongliang Lü

Hongliang Ren

Hongliang Zhou

Hongmin $\mathrm{Wu}$

Hongming $\mathrm{Xu}$

Hongpeng $\mathrm{Wu}$

Hongpeng Zhang

Hongseok An

Hong-Seok Park

Hongsheng Li

Hongsun Guo

Hongtak Lee

Hongtian Chen

Hongwei Qu

Hongwei Wang

Hongwu Wang

Hongxin Huang

Hongyin Shi

Hongyu $\mathrm{Hu}$

Hongyu Yu

Hongyu Zhao

HongYu Zheng
Hongyuan Huo

Hongyue Sun

Hongyun Liu

Hong-Zen Yeh

Hongzhou Yang

Hoon Oh

Hoon Sohn

Horácio Oliveira

Horacio Paggi

Horatiu Stefanie

Horn-jiunn Sheen

Hosam Al-Samarraie

Hosameldin Ahmed

Hosang Ahn

Hoseon Lee

Hosik Seok

Hoson Lam

Hossain Md. Bellal

Hossam A. Gabbar

Hossam Selim

Hossein Bagheri

Hossein Fotouhi

Hossein Ghaemi

Hossein Hassani

Hossein Hondori

Hossein Mardani

Hossein Sahour

Hossein Tabatabaei-Jafari

Hossein Taheri

Hosseon Rabbani

Hou-bin Li

Houbing Song

Houpu Li

Houssam Salmane

Howard Matis

Howuk Kim

Ho-Yong Ahn

Hriday Bavle

Hridesh Kumar Mishra

Hristos Anastassiu

Hrvoje Glavas

Hrvoje Jakovac

Hrvoje Kalinić

Hrvoje Karninčić

Hrvoje Kutnjak

Hsi Lien Hsiao

Hsiang-Chen Wang

Hsiang-Chieh Chen

Hsiang-Chieh Lee

Hsiao-Chuan Liu

Hsiao-Chun $\mathrm{Wu}$ 
Hsiao-Ping Tsai

Hsiao-Ting Tseng

Hsiao-Tung Chang

Hsieh Bao-Yu

Hsien-Pin Hsu

Hsien-Tsai Wu

Hsien-Wei Yeh

Hsi-Hsir Chou

Hsin Hsiu

Hsin-Han Chiang

Hsin-Jang Shieh

Hsin-Ta Chiao

Hsinwen Ting

Hsin-Yi Kathy Cheng

Hsin-yi Wen

Hsiu-Ming Wu

Hsuan-Ling Kao

Hsuan-Shih Lee

Hsueh-chun Lin

Hsueh-Hsien Chang

Hsueh-wen Chow

Hsueh-Wen Tseng

Hsu-Heng Yen

Hu Ding

$\mathrm{Hu} \mathrm{He}$

Hu Sun

Hu Xiong

Hua Li

Hua Shi

Hua Sun

Hua Xu

Hua Zou

Huachun $\mathrm{Wu}$

Huachun Zhou

Huafei Du

Huafeng Liu

Huai-Kuei Wu

Huaiyu Yang

Huajun Song

Huakun Huang

Hualong Xie

Huan He

Huan Liu

Huan Ni

Huan Pang

Huan Wang

Huan Zhao

Huang Li

Huangke Chen

Huan-Hsuan Hsu

Huanyu (Larry) Cheng
Huanyu Cheng

Hua-Pin Chen

Huaqing Cui

Huaqing Wang

Huawei Jiang

Huawei Liang

Huaxiang Wang

Huaxing Jiang

Hubin Zhao

Hucheng Sun

Huei-Yung Lin

Huey Hoon Hng

Huey-Ming Tzeng

Hugh J. Byrne

Hugh Lee

Hugo A. Loaiciga

Hugo Almeida Ferreira

Hugo André

Hugo Entradas Silva

Hugo F. Martins

Hugo F. Posada-Quintero

Hugo Fernando Posada-Quintero

Hugo Ferreira

Hugo G. Espinosa

Hugo J. Avila-Paredes

Hugo José Nogueira Pedroza Dias Mello

Hugo Rojas-Chávez

Hugo Saner

Hugo Sarmento

Hugues Pretrel

Huh Tae Myung

Hui Chen

Hui Huang

Hui Jiang

Hui Li

Hui Ma

Hui Sun

Hui Wang

Hui Yang

Hui Zhang

Hui Zheng

Huie Zhu

Huifang Li

Hui-Hsin Hsiao

Huijia Li

Huiling Tai

Huilong Zhang

Huimin Liu

Huimin Lu

Huimin Shen

Huimin Zhang 
Huixiang $\mathrm{Wu}$

Huixuan Fu

Humberto C. Godinez

Humberto Lobato-Morales

Humberto Martínez-Barberá

Humberto Sossa

Hung Cao

Hung Nguyen

Hung Soo Kim

Hung Tuan Nguyen

Hung-Chi Chu

Hung-Huan Liu

Hung-Jr Shiu

Hung-Shing Chen

Hung-Vu Tran

Hung-Yin Lin

Hung-Yu Chien

Hunter Bachman

Huong Thu Nguyen

Huseyin Kusetogullari

Hüseyin Polat

Husnain Sherazi

Hussam Hussein

Huxuan Wang

Hwajeong Seo

Hwang Seung-Hoon

Hwang Yi

Hwang-Cherng Chow

Hyejeong Ryu

Hyeon Seok An

Hyeong-U Kim

Hyeon-June Kim

Hyogon Kim

Hyojung Hyun

Hyosang Yoon

Hyoseok Yoon

Hyoungmin So

Hyowon Ban

Hyoyoung Jeong

Hyuck Jin Lee

Hyuk-Jae Roh

Hyun Cheol Kim

Hyun Ho Lee

Hyun Kwon

Hyunchae Chun

Hyunchul Kang

Hyunchul Shin

Hyun-Deok Kim

Hyung D. Bae

Hyung Seok Kim

Hyungchul Yoon
Hyungjong Kim

Hyung-Joon Seo

Hyung-Tae Kim

Hyun-Ho Choi

Hyunjoo Jenny Lee

Hyunjung Lee

Hyunseok Seo

Hyunsoo Lee

Hyunwoo Hwangbo

Iacopo Tamellin

Iain Collings

Ian A. Grout

Ian Cleland

Ian Curthoys

Ian Daniel Leroux

Ian Davis

Ian McAndrew

Ian Moss

Ian Williams

Iaroslav Gnilitskyi

Iatrellis Omiros

Ibrahim Abdulhalim

Ibrahim Furkan Ince

Ibrahim Yitmen

Ibukun Gabriel Awolusi

Ichiro Fujita

Ichiro Yamada

Ickjin Kwon

Iclia Villordo Jimenez

Ic-Pyo Hong

Idiano D'Adamo

Idiano D'Adamo

Idilia Batchkova

Idongesit Ekerete

Idowu Paul Okuwobi

Idris Ahmed

Ieva Meidute-Kavaliauskiene

Ieva Plikusiene

Ievgeniia Kuzminykh

Iftekhar Khan

Iga Hołyńska-Iwan

Iga Rybicka

Ignacio Bravo

Ignacio Fernández-Hernández

Ignacio Javier Pérez

Ignacio Lijarcio

Ignacio Llamas-Garro

Ignacio Rodríguez-Rodríguez

Ignacio Roig

Ignas Budvytis

Ignas Grigelionis 


\begin{tabular}{|c|c|}
\hline Ignasi Corbella & Ilona Jacyna-Gołda \\
\hline Igor A. Konyakhin & Ilona Pawełoszek \\
\hline Igor Aleinov & Il-Seuk Koh \\
\hline Igor Bufetov & Ilshat Khasanshin \\
\hline Igor Buzalewicz & Iltai (Isaac) Kim \\
\hline Igor Bychkov & Il-Woo Nam \\
\hline Igor Chunchuzov & Ilya N. Pavlov \\
\hline Igor Filanovsky & Ilya Turchin \\
\hline Igor Fürstner & Ilya Yakavets \\
\hline Igor Gruic & Imad Rida \\
\hline Igor Korobiichuk & Imadeddine Azzouz \\
\hline Igor Loboda & Iman Abaspur Kazerouni \\
\hline Igor Ogashawara & Iman Heidarpour Shahrezaei \\
\hline Igor Pusnik & Iman Izadgoshasb \\
\hline Igor Ružić & Iman Soltani Bozchalooi \\
\hline Igor Saenko & Imena Rexhepi \\
\hline Igor Savin & Immihan Ceren Yasa \\
\hline Igor Tomicic & Imran Ashraf \\
\hline Igor Vujović & Imran Baig \\
\hline Ihr Jörg Schäfer & Imran Khan Niazi \\
\hline Ihsan Ali & Imran Memon \\
\hline Iker Pastor-López & Imran Niazi \\
\hline Ik-Hyun Youn & Imran Tasadduq \\
\hline Ikjune Yoon & Imre Kiss \\
\hline Ikram Ud Din & Imtiaz Ahmed \\
\hline Il Song Han & Imtiaz Mahmud \\
\hline Ila Maltese & Im-Yeong Lee \\
\hline Ilaria Bortone & In Soo Lee \\
\hline Ilaria Campioni & Inacio Yano \\
\hline Ilaria De Munari & Inayat Ur Rahman \\
\hline Ilaria Ferrando & Inder Gupta \\
\hline Ilaria Mileti & Ines Delfino \\
\hline Ildiko Mankova & Ines Kožuh \\
\hline Ildiko Szanto & Ines Obradović \\
\hline Ileana Bogdan & Inés Sittón-Candanedo \\
\hline Ileana Hamburg & Inga Morkvenaite-Vilkonciene \\
\hline Ileana Nicoleta Popescu & Inge Wiekenkamp \\
\hline Ileana Pirovano & Ingmar Müller \\
\hline Il-Gu Lee & Inhee Lee \\
\hline Ilia N. Ivanov & In-ho Ra \\
\hline Ilia Safonov & Inhong Song \\
\hline Ilias Mavroidis & Iñigo Barandiaran \\
\hline Ilias N. Lymperopoulos & Iñigo Cuiñas \\
\hline Ilias Politis & Inma Mohino-Herranz \\
\hline Ilias Theodorakopoulos & Inmaculada Aparicio \\
\hline Ilias Travlos & Inmaculada Romero Gil \\
\hline Ilie Valentin Mihai & Innokentiy Kastalskiy \\
\hline I-Lin Tsai & Inseop $\mathrm{Na}$ \\
\hline Ilja Croijmans & Insoo Kim \\
\hline Ilke Coskun Benlidayi & Intisar Ali Sajjad \\
\hline Ilkka Nissinen & Inwook Hwang \\
\hline
\end{tabular}


Inyong Kwon

Inż. Bartosz Olejnik

Ioan Burda

Ioan Nicolaescu

Ioan Petean

Ioan Silea

Ioan Stefan Sacala

Ioan Susnea

Ioan Tudosa

Ioan Ungurean

Ioana Dana Alexa

Ioana Marcu

Ioana Opris

Ioanis Nikolaidis

Ioanna Roussaki

Ioannis Chatzigiannakis

Ioannis D. Moscholios

Ioannis Faraslis

Ioannis Karamitsos

Ioannis Kioutsioukis

Ioannis Kosmas

Ioannis Kostavelis

Ioannis P. Gravas

Ioannis Paliokas

Ioannis Samaras

Ioannis T. Georgiou

Ioannis Tsoulos

Ioannis Vardiambasis

Ioannis Vourganas

Iole Venditti

Ion Dan Borcia

Ion Emilian Radoi

Ion Giosan

Ion Sandu

Ion Vornicu

Ionel Staretu

Ionel Zagan

Ionica Oncioiu

Ionut Cristi Nicu

Ionut Daniel Geonea

Ionut Emil Iacob

Ionut Schiopu

Iordana Astefanoaei

Iosif Sifakakis

Iosif Szeidert

Iosif-Vasile Nemoianu

Ipin Chang

Ippokratis Sartzetakis

Iren E. Kuznetsova

Iren Valova

Irena Fryc
Irena Ištoka Otković

Irena Ivanišević

Irena Jekova

Irena M. Hlaváčová

Irena Orović

Irene Daskalopoulou

Irene Nutini

Irene Torres-Sanchez

Ireneusz Dominik

Ireneusz Kubiak

Irenilza De Alencar Nääs

Irfan Ahmad Khan

Irfan Khan

Irina B. Vendik

Irina D. Yushina

Irina G. Palchikova

Irina Georgiana Mocanu

Irina Mocanu

Irina Neaga

Irina Ontel

Irina Rabaev

Irina Severin

Irina Voytyuk

Irina Yatskiv

Irineo L. López-Cruz

Irineu De Brito Junior

Iris Kico

Irmantas Kašalynas

Irwin A. Quintela

Isaac Macwan

Isaac Triguero

Isabel Castro

Isabel Jesus

Isabel Perez De Vargas Sansalvador

Isacco Gualandi

Isaías González Pérez

Ishan Joshi

Ishtiaq Ahmad

I-Shyan Hwang

Islam Ashry

Islam Md Rizwanul Fattah

Islam Mosa

Ismail Butun

Ismail Fidan

Ismini E. Papageorgiou

Israel Casado-Hernández

Israel David Hinostroza Sáenz

Israel Donizeti De Souza

Israel Hinostroza

Israel Leyva-Mayorga

Israel Martin-Escalona 
Israel Miguel Andrés

Israr Ullah

István Budai

Istvan Elek

István Grigorszky

Istvan Gyongy

István Pálinkó

Istvan Rajta

István Vassányi

Italo Atzeni

Itay Barel

Itay Barnea

Itshak Tkach

Itthipon Jeerapan

Itzamá López-Yáñez

Itzel Nunez

Itziar Alonso González

Itziar Cabanes

Itzik Klein

Iulian Ciocoiu

Iuliia Novoselova

Iurii Semenov

Ivailo Pandiev

Ivan A. Denisov

Ivan A. Parinov

Ivan Adriyanov Nikolov

Ivan Aleksi

Ivan Bobrinetskiy

Ivan Brak

Ivan Bratchenko

Ivan Casella

Ivan Chavdarov

Ivan Cruz-Aceves

Ivan Cundrle

Ivan Cvitic

Ivan Cvitić

Ivan D. Castro

Ivan Derkach

Ivan Dunđer

Ivan Forenbacher

Ivan Giannoccaro

Ivan Grgić

Ivan Gruber

Iván Herrera-Peco

Iván Herrero-Durá

Ivan Iudice

Ivan Ivanovich Stoikov

Ivan Izonin

Ivan Kotuliak

Ivan Lim Chen Ning

Ivan Lipuzhin
Ivan Lorencin

Ivan Marić

Ivan Mezei

Ivan Miguel Pires

Ivan Miguel Serrano Pires

Ivan Miskulin

Ivan Oropeza-Perez

Ivan Petrunin

Ivan Petryshynets

Ivan Pires

Ivan Plaščak

Ivan Prochazka

Ivan Puchades

Ivan Puente

Ivan Shorubalko

Ivan Simões Gaspar

Ivan Tomasic

Ivan Vaccari

Ivan Virgala

Ivan Vujaklija

Ivan Zajačko

Ivana Barisic

Ivana Gadjanski

Ivana Tlak Gajger

Ivanjko Edouard

Ivanovitch Silva

Iveta Vasková

Ivica Stančerić

Ivo Buttinoni

Ivo Dobrev

Ivo Draganov

Ivo Iliev

Ivo Stancic

Ivor Nissen

Iwaki Akiyama

Iwona Grabowska

Iwona Grobelna

Iwona Kochańska

Iwona Komorska

Iwona Paprocka

Izaak Neveln

Izabela Barszczewska-Rybarek

Izabela Gołębiowska

Izabela Karsznia

Izabela Szarmach

Izabella Lecka

Izadyar Tamadon

Izidor Mlakar

J Randall Moorman

J. A. De Jesús Osuna-Coutiño

J. Apolinar Muñoz Rodriguez 
J. Ernesto Solanes

J. Gabriel Ortega-Mendoza

J. Gaxiola-Camacho

Jaakko Hakulinen

Jaan Ojarand

Jaana Parviainen

Jaap Velthuis

Jabar H. Yousif

Jabed Chowdhury

Jacek Caban

Jacek Dawidowicz

Jacek Gebicki

Jacek Górka

Jacek Kesik

Jacek Łukasz Wilk-Jakubowski

Jacek Marciniak

Jacek Marczewski

Jacek Matys

Jacek Misiurewicz

Jacek Nowakowski

Jacek Oskarbski

Jacek Pietraszek

Jacek Polechoński

Jacek Stepien

Jacek Tomków

Jacek Wojtanowski

Jacek Wojtas

Jacek Woźniak

Jacek Wytrębowicz

Jacinto Santamaría-Peña

Jack Bishop

Jack Merrin

Jack Wang

Jackie Reznik

Jacky Loo

Jacob Grazer

Jacob Hinkel-Lipsker

Jacob Sosnoff

Jacopo Aguzzi

Jacopo Catani

Jacopo Lamanna

Jacopo Pavan

Jacque Kelly

Jacqueline Walker

Jacques Penders

Jader Cabral

Jae Hee Kim

Jae Hong Shim

Jae Hoon Lee

Jae Joon Kim

Jae Min Lee
Jae Woong Lee

Jae Youn Hwang

Jaehan Koh

Jae-Han Park

Jaehong Kim

Jae-Hoon Kim

Jaehwa Park

Jaehyeok Doh

Jaehyuk Choi

Jaehyun Jin

Jae-Hyun Kim

Jaehyun Park

Jaehyung Yu

Jaejong Park

Jae-Myung Ryu

Jaepil Ko

Jaerock Kwon

Jaesoon Choi

Jaesun Lee

Jaesung Park

Jaewon Yang

Jaewoo Lee

Jaeyong Kang

Jaeyoung Lee

Jaeyoung Shin

Jaeyul Lee

Jafrancesco David

Jagajjit Sahu

Jagannath Aryal

Jagannathan Sarangapani

Jaime Alvarez-Quintana

Jaime Andres Garcia

Jaime Duque Domingo

Jaime Lloret

Jaime Martinez-Castillo

Jaime Moreno

Jaime Muñoz-Arteaga

Jaime Ortegon

Jaime Ramis Soriano

Jaime Villate

Jain-Shing Liu

Jair Cervantes

Jaka Sodnik

Jakub Ciazela

Jakub Horák

Jakub Kostelecký

Jakub Krejčí

Jakub Lengiewicz

Jakub Markiewicz

Jakub Nalepa

Jakub Nedbal 


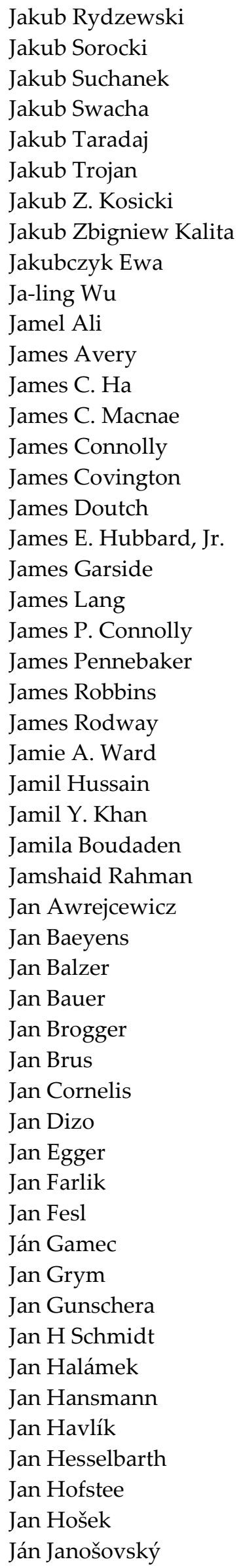

Jakub Rydzewski

Jan Kazak

Jan Kelner

Jan Koltermann

Jan Komarek

Jan Konarski

Jan Kouba

Jan Kř́ž

Jan L. Cieśliński

Ján Labuda

Ján Labun

Jan Latal

Jan Mandel

Jan Mares

Jan Masajada

Jan Mietzner

Jan Mrazek

Jan Mućko

Jan Muzik

Jan Nikodem

Jan Nissinen

Ján Papaj

Jan Pennekamp

Jan Petersen

Ján Pitel'

Jan Saliga

Jan Schmitt

Jan Schür

Jan Sikora

Jan Strobl

Jan Studziński

Jan Suchanicz

Jan Szatyłowicz

Jan Tomastik

Jan Trmal

Jan Turan

Ján Vachálek

Jan Vilhelm

Jan Vrba

Jan Wajs

Jan Warczek

Jan Wendel

Jan Wietrzykowski

Jana Antalíková

Jana Houser

Jana Kalbacova Vejpravová

Jana Striova

Janaina Antonino Pinto

Janaka Ekanayake

Jane Courtney

Jane Jean Kiam

Janet Light 


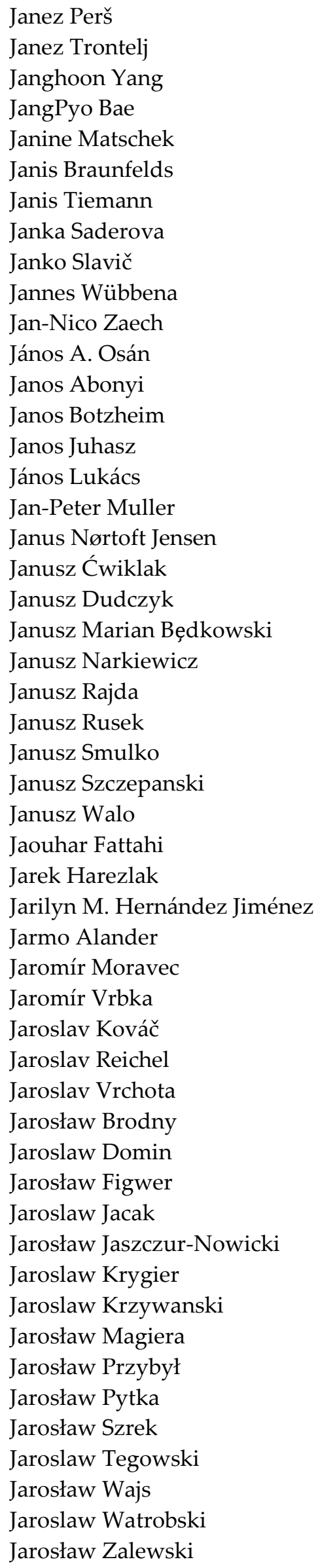

\author{
Jarosław Zubrzycki \\ Jaroslaw Zygarlicki \\ Jascha Kolberg \\ Jasenka Gajdoš Kljusurić \\ Jasmina Lukinac \\ Jasmina Vidic \\ Jason Barker \\ Jason Brown \\ Jason Friedman \\ Jason G. Zeibel \\ Jason J. Gorman \\ Jason M. Hite \\ Jason M. Whyte \\ Jason Poulos \\ Jason Rambach \\ Jasper Reenalda \\ Jasreman Singh \\ Jasvir Dalal \\ Jaume Anguera \\ Jaume Bacardit \\ Jaume Coll-Font \\ Jaume Francisco-Pascual \\ Jaume Verd \\ Javad Rostami \\ Javad Zarrin \\ Javier A. De La Tejera \\ Javier Burgués \\ Javier Díez-González \\ Javier E. Contreras-Reyes \\ Javier García Castellano \\ Javier Garcia-Casado \\ Javier Garcia-Guzman \\ Javier Gomez-Pilar \\ Javier González-Rocha \\ Javier López-Randulfe \\ Javier Martínez Torres \\ Javier Medina Quero \\ Javier Monroy \\ Javier Moreno-Valenzuela \\ Javier Nuno \\ Javier Pereira Loureiro \\ Javier Pérez Fernández \\ Javier Ramos-Ortega \\ Javier Reina-Tosina \\ Javier Riedemann \\ Javier Roales \\ Javier Rocher \\ Javier Rodrigo-Ilarri \\ Javier Ros \\ Javier Rubio Loyola \\ Javier Sanchez Galan
}


Javier Silvestre

Javier Silvestre-Blanes

Javier Toledo

Javier Urraca

Javier Vázquez

Javier Vázquez-Castillo

Javier Villalba

Jawad Ahmad

Jawed Qureshi

Jay A. Weitzen

Jay L. Nadeau

Jay Wadhawan

Jayachandran Venkatesan

Jaydip Desai

Jayme Garcia Arnal Barbedo

Jayne $\mathrm{Wu}$

Je Hyung Jung

Jea Soo Kim

Jea Uk Lee

Jean Claude De Mauroy

Jean D. Hallewell Haslwanter

Jean Jacques Loiseau

Jean Michel Létang

Jean Sequeira

Jean-Baptiste Renard

Jean-Baptiste Thomas

Jean-Charles Beugnot

Jean-Charles Bolomey

Jean-Claude Charr

Jean-Francois Vuillaume

Jean-Jacques Ponciano

Jean-Luc Seguin

Jean-Marc Ribeiro

Jean-Marc Tulliani

Jean-Marc Zingg

Jean-Marie Sleewaegen

Jean-Michel Friedt

Jean-Michel Martinez

Jean-Michel Romano

Jean-Paul Calbimonte

Jean-Paul Linnartz

Jean-Philippe Ansermet

Jean-Philippe Didon

Jean-Philippe Roberge

Jean-Philippe Tarel

Jean-Pierre Barriot

Jean-Pierre Corriou

Jean-Pierre Huignard

Jean-Pierre Jessel

Jean-Pierre Raulin

Jee Woong Choi
Jeeyoung Lim

Jefferson Araujo

Jefferson Santos De Gois

Jeffrey A. Tuhtan

Jeffrey Buckley

Jeffrey C. F. Ho

Jeffrey Kilby

Jeffrey S. Hallam

Jeffrey S. Stevenson

Jehad Ali

Jehan-Antoine Vayssade

Jehwan Hwang

Jelena Kilić Pamuković

Jelena Muncan

Jen-Chieh Liu

Jen-Chuan Tung

Jeng-Ren Duann

Jeng-Shin Sheu

Jennifer Davies

Jennifer Gubitosa

Jennifer Howcroft

Jennifer M. Vojtech

Jennifer Symons

Jenn-Kaie Lain

Jenny Benois-Pineau

Jenny Huang

Jenny Pagge

Jens Christian Andersen

Jens Hartmann

Jens Hiller

Jens Jensen

Jens Kirchner

Jens M. Hovem

Jens Muehlsteff

Jens Neu

Jens V. Fischer

Jenu Chacko

Jeon Lee

Jeong Min Kang

Jeong Ryeol Choi

Jeong Su Lee

Jeongguk Kim

Jeonghoon Kwak

Jeonghyun Kim

Jeong-Jung Kim

Jeongmoo Huh

Jeongsik Choi

Jeongyeup Paek

Jeremie Houssineau

Jeremie Sublime

Jeremy Adler 


Jérémy Vézinet
Jeroen Bergmann
Jeroen Degerickx
Jerome Antoni
Jérôme Coste
Jérôme De Miras
Jérôme Idier
Jérome Luc
Jérôme Molimard
Jérôme Rossignol
Jérôme Tchoufang Tchuindjang
Jérôme Tissier
Jerry Jialie Shen
Jerzy A. Sładek
Jerzy Bakunowicz
Jerzy Balicki
Jerzy Hapanowicz
Jerzy J. Jasielec
Jerzy J. Langer
Jerzy Jozwik
Jerzy Konorski
Jerzy Majka
Jerzy Mikulski
Jerzy Potencki
Jerzy Siuzdak
Jerzy Świątek
Jerzy Winczek
Jerzy Wtorek
Jesper Rasmussen
Jesse Yoe Rumbo Morales
Jessica Ramella-Roman
Jesús Ortiva-Pás Pastor
Jesús Pérez-Ríos
Jessie T. Childs
Jesus Cruz-Garza
Jesús De Vicente Y Oliva
Jesús Donaire-Avila
Jesus E. Velazquez-Perez
Jesus Enrique Sierra
Jesús Fernández-Hernández
Jesus G. Cruz-Garza
Jesús Grajal De La Fuente
Jesús López Belmonte
Jesus Lozano

Jérémy Vézinet Jeroen Bergmann Jeroen Degerickx Jerome Antoni Jérôme Coste Jérôme De Miras Jérôme Idier Jérome Luc Jérôme Molimard Jérôme Rossignol Jérôme Tissier Jerry Jialie Shen Jerzy A. Sładek Jerzy Bakunowicz Jerzy Balicki Jerzy Hapanowicz Jerzy J. Jasielec Jerzy Konorski Jerzy Majka Jerzy Mikulski Jerzy Potenck Jerzy Siuzdak Jerzy Świątek Jerzy Winczek Jerzy Wtorek Jesse Yoe Rumbo Morales Jessica Ramella-Roman Jessie T. Childs Jesus Cruz-Garza Jesús De Vicente Y Oliva Jesús Donaire-Avila Jesus E. Velazquez-Perez Jesus Enrique Sierra Jesús Fernández-Hernández Jesus G. Cruz-Garza Jesús Grajal De La Fuente Jesús López Belmonte Jesus Lozano Jesús M. Gómez-de-Gabriel Jesus Manuel Gomez-de-Gabriel Jesús Manuel Nieto García Jesus Mora-Rodriguez Jesús Oliva-Pascual-Vaca
Jesus Roberto Millan Almaraz Jesús Salido Tercero Jesus Salinas Jesus Sanchez-Gomez Jesús Sergio Artal-Sevil Jesus Tejada Jesús-Ignacio Prieto Jetmir Haxhibeqiri Jevgenijus Toldinas Jeyeon Kim JH Cho Jhantu Kumar Saha Jhe-Syuan Lai Jhon Castaño-Osorio Jhon Silva-Castro Ji Bo Wen Ji Fan Ji Han Jia Jin Jia Li Jia Rong Jia Shi Jia Uddin Jia $W u$ Jia $\mathrm{Xu}$ Jiabao Yu Jiachao Zhang Jiachen Yang Jiachou Wang Jiafeng Gu Jiafeng Xie Jiafeng Yao Jiahao Yan Jiahao Zhong Jiahong Chen Jiahuan Jiang Jia-Jung Wang Jiakai Li Jiali Yin Jialun Liu Jiamin Sun Jiaming Liang Jiaming $\mathrm{Na}$ Jian Gao Jian Ge Jian Huang Jian Jiao Jian Kang Jian Ma Jian Sun Jian Wan 


Jian Wang
Jian Wei Tay
Jian Weng
Jian Xu
Jian Xue
Jian Yang
Jian Zhang
Jian Zhou
Jianbin Gao
Jianbin Qiu
Jianbin Xin
Jianbing Xie
Jianbo Qi
Jianbo Wu
Jiancheng Tao
Jiande Sun
Jiandong Ren
Jianfa Zhang
Jianfeng Li
Jianfeng Ping
Jianfeng Ren
Jianfeng Wu
Jianfeng Zhao
Jiang Liu
Jiang Zhao
Jiangbo Pu
Jiangbo Xi
Jiangcheng Li
Jianghai Xia
Jianghong Mao
Jianglei Di
Jiangsheng Gui
Jiangtao Xu
Jianguo Chen
Jianguo Ding
Jianguo Liu
Jianguo Lu
Jiangwei Tian
Jianhai Zhang
Jianhua Liu
Jianhua Wu
Jianing Li
Jia-Ning Luo
Jian-Jang Huang
Jianjia Wang
Jianjun Ma
Jiankang Wu
Jiankun Wang
Jianlai Chen
Jianlin Guo
Jianming Wen

Jian Wang

Jian Wei Tay

Jian Weng

Jian Xu

Jian Xue

Jian Yang

Jian Zhang

Jian Zhou

Jianbin Gao

Jianbing Xie

Jiancheng Tao

Jiande Sun

Jiandong Ren

Jianfa Zhang

Jianfeng Li

Jianfeng Ping

Jianfeng Ren

Jianfeng Wu

Jianfeng Zhao

Jiang Liu

Jiang Zhao

Jiangbo $\mathrm{Pu}$

Jiangbo $\mathrm{X}_{\mathrm{i}}$

Jiangcheng Li

Jianghai Xia

Jianghong Mao

Jianglei Di

Jiangsheng Gui

Jiangtao $\mathrm{Xu}$

Jianguo Chen

Jianguo Ding

Jianguo Liu

Jianguo Lu

Jiangwei Tian

Jianhai Zhang

Jianhua Liu

Jianhua Wu

Jianing Li

Jia-Ning Luo

Jian-Jang Huang

Jianjia Wang

Jianjun Ma

Jiankang Wu

Jiankun Wang

Jianlai Chen

Jianming Wen
Jianming Zhang

Jianping Li

Jianqi Wang

Jianqiang Qian

Jianqing $\mathrm{Wu}$

Jiantao Yao

Jianwu Fang

Jianwu Zhang

Jianxi Liu

Jianxin Jia

Jianxin Yi

Jianxing Liu

Jianxiong Zhu

Jianyi Liu

Jianyu Fu

Jian-Zhang Chen

Jianzhen $\mathrm{Ou}$

Jianzhong Li

Jianzhong Zhang

Jiaqi Han

Jiaqi Yang

Jiaqing Xiong

Jiarui Huang

Jiarui Lin

Jia-Shiun Chen

Jiawang Chen

Jiawei Chen

Jiawei Huang

Jiawei Xiang

Jiawen Xu

Jiaxin Liu

Jiaxing Che

Jiayi Ma

Jiayong $\mathrm{Yu}$

Jiayu Li

Jiayuan $\mathrm{He}$

Jiba Dahal

Jibin Zheng

Jibran Khaliq

Jichao Hong

Jichiang Tsai

Jidong Yang

Jie Cao

Jie Cheng

Jie Cui

Jie Deng

Jie $\mathrm{Hu}$

Jie Hua

Jie Huang

Jie Li

Jie Ling 


\begin{tabular}{|c|c|}
\hline Jie Qi & Jing Jin \\
\hline Jie Yang & Jing Li \\
\hline Jie Zhang & Jing Liu \\
\hline Jiejian Dai & Jing Selena He \\
\hline Jiewu Leng & Jing Teng \\
\hline Jieyun $\mathrm{Wu}$ & Jing Tian \\
\hline Jifang Tao & Jing Wang \\
\hline Jifeng Liu & Jing Wei \\
\hline Jihong Kim & Jing Xu \\
\hline Jihoon Kim & Jing Yan \\
\hline Jihoon Lee & Jing Yang \\
\hline Jihoon Moon & Jing Yue \\
\hline Ji-Hoon Yun & Jing Zhang \\
\hline Jihun Kim & Jing Zhou \\
\hline Jihyoung Cha & Jingang Zhong \\
\hline Jihyun Bae & Jingbiao Chen \\
\hline Jijun Ren & Jingbo Zhao \\
\hline Jiliang Zhang & Jingchen Wang \\
\hline Jimin Maeng & Jingcheng Zhou \\
\hline Jimmie Miller & Jing-Chie Lin \\
\hline Jimmy Olsson & Jingfei Liu \\
\hline Jims Marchang & Jingfeng Huang \\
\hline Jin Chen & Jing-Hui Qiu \\
\hline Jin $\mathrm{He}$ & Jingjing $\mathrm{He}$ \\
\hline Jin Kyu Gahm & Jingjing Luo \\
\hline Jin Li & Jingjing Xu \\
\hline Jin Liang & Jinglei Tang \\
\hline Jin Ning & Jinglin Zhang \\
\hline Jin Qi & Jinglong Chen \\
\hline Jin Sik Kim & Jingru Yi \\
\hline Jin Su Jeong & Jingshui Huang \\
\hline Jin Tao & Jingsong Li \\
\hline Jin Wang & Jinguang Cai \\
\hline Jin Wen & Jingya Li \\
\hline Jin Yan & Jingyu Wang \\
\hline Jin Yang & Jingyu Yang \\
\hline Jin Young Lee & JingZheng Huang \\
\hline Jin Zhang & Jin-Hak Yi \\
\hline Jinao Zhang & Jinho Yoon \\
\hline Jinbo Wu & Jinhong Guo \\
\hline Jinchao Chen & Jinhua Sun \\
\hline Jinchao Tong & Jinhua Yu \\
\hline Jinchen Ji & Jinhyeong Kwon \\
\hline Jincheng Lei & Jinjin Yan \\
\hline Jindong Song & Jinkui Chu \\
\hline Jindřich Libovický & Jinlian $\mathrm{Hu}$ \\
\hline Jindrich Liska & Jin-Liang He \\
\hline Jinfeng Li & Jinling Zhao \\
\hline Jinfu Liu & Jinlong Fan \\
\hline Jing Chen & Jinming Liu \\
\hline Jing Fan & Jinnan Zhang \\
\hline
\end{tabular}


Jinn-Min Yang

Jinping Sun

Jinquan Liu

Jinsheng Yang

Jinsil Lee

Jinsong $\mathrm{Wu}$

Jinsuk Baek

Jinwei Sun

Jinwen Ma

Jinwoo Jang

Jin-Woo Jeong

Jinwoo Shin

Jinwook Kim

Jinwu Kang

Jinxi Guo

Jinxin He

Jinxin Liu

Jinxing Qiu

Jinyang Du

Jinyang Liang

Jinyang Zhang

Jinyi Li

Jin-Yuan Wang

Jinzu Ji

Jiri Barek

Jiři Erhart

Jiř́ Janda

Jiř́ Krejsa

Jiri Petrzela

Jiří Pfleger

Jiri Plasek

Jiř́i Přibil

Jiri Tesar

Jiří Vackář

Jiseok Lim

Jitae Shin

Jitang Fan

Jitka Kumhálová

Jiuhou Lei

Jiuk Jang

Jiulu Gong

Jiun-in Guo

Jiun-Jian Liaw

Jiun-Yu Sung

Jiushuai Xu

Jivitesh Sharma

Jiwei Huang

Jiwen Cui

Ji-Won Seo

Jiwoo Kang

Ji-Wook Yoon
Jiyong $\mathrm{Hu}$

Jiyoung Jung

Jize Zhang

Jizhong Song

Jo Verhaevert

Joab R. Winkler

Joachim Clemens

Joachim Enders

Joachim Ingwersen

Joachim Jonuscheit

Joachim Wiest

Joan Bas

Joan Bordoy

Joan Cabestany

Joan Claudi Socoró

Joan Leal-Blanquet

Joan Navarro Martín

Joan Pons-Llinares

Joan Ramon Casas

Joan Rosselló

Joana Costa

Joana Figueiredo

Joana Guedes

Joana M. Warnecke

Joan-Francesc Fondevila-Gascón

Joanna Adamczyk

Joanna Borowiec

Joanna Cabaj

Joanna Dipnall

Joanna Grygierek

Joanna Iwaniec

Joanna Janicka

Joanna Jaworek-Korjakowska

Joanna Konopińska

Joanna Kos-Łabędowicz

Joanna Liszkowska

Joanna Maria Dulińska

Joanna Ortyl

Joanna Paciorek-Sadowska

Joanna Przeździecka-Dołyk

Joanna Sekulska Nalewajko

Joanna Sobiak

Joanna Zajda

Joao A. Lorenzzetti

João Abrantes

João Almeida

João Barata

João C. Neves

João Calado

Joao Carlos Amaro Ferreira

João Carlos Ferreira 
João Castelhano

João Coelho

João Felício

Joao Ferreira

João Filipe Fernandes Lindo Simões

João Flávio Da Silveira Petruci

João Kleinschmidt

João Leitão

João M. F. Calado

João M. L. P. Caldeira

João Miguel da Costa Sousa

Joao Miguel Sousa

Joao Moreira

João Mouro

Joao Nuno Matos

João Nuno Matos

Joao Palotti

João Patrício

João Paulo Barraca

João Paulo Carmo

João Paulo Carvalho Lustosa Da Costa

Joao Paulo Flores

João Pereira

João Pinto Coelho

João Reis

João Ribeiro Pinto

João Rocha Vaz

Joao Ruivo Paulo

João Silva Sequeira

João Vilas-Boas

Joaquim Azevedo

Joaquim C.G. Esteves Da Silva

Joaquim Chaler

Joaquim De Moura

Joaquim Ferreira

Joaquim Miguel Maia

Joaquim Minguella-Canela

Joaquim Sousa

Joaquim Tinoco

Joaquin Aranda

Joaquín Gayoso-Cabada

Joaquin Gonzalez

Joaquin L. Herraiz

Joaquín Luque

Joaquin Martinez

Joaquin Paez-Moguer

Joaquin Sanchiz

Joaquín Torres-Sospedra

Jobst Wurl

Joceli Mayer

Jochen Hiller
Jochen Lang

Jochen Manara

Jochen Seitz

Joe Wiart

Joel Antonio Trejo Sánchez

Joël Colloc

Joël Lemaire

Joep Van Den Broeke

Johan Casselgren

Johan J. Estrada-López

Johan Jair Estrada-López

Johan Markdahl

Johan N Van Der Meer

Johan Van Der Meer

Johann Kastner

Johann Marton

Johannes Betz

Johannes Fresner

Johannes P. Van Dijk

Johannes Roths

Johannes Vorwerk

Johannes W. Passand

Johannes Winter

Johari Yap Abdullah

John A. Mercer

John Abela

John Adamovics

John Allen

John C.-C. Lu

John D. Kechagias

John Dermentzoglou

John Enright

John G. Kellett

John Gialelis

John Gountas

John Hansen

John J. Guers

John Jairo Villarejo Mayor

John Jelonnek

John M. Abendroth

John M. Acken

John McCamley

John McNutt

John Michael Retterer

John Murray-Bruce

John Nassour

John Rebula

John S. Mitchell

John Saffell

John Thomas

John Tsiligkaridis 
John Withers Walker

John-Lewis Zinia Zaukuu

Joice Mathew

Jolanta Karpowicz

Jolanta Pauk

Jon Ashley

Jon Glenn Gjevestad

Jon Legarda Macon

Jon Youn

Jonas H. Osório

Jonas Matijošius

Jonas Neuser

Jonatan Lerga

Jonathan Brembeck

Jonathan Engel

Jonathan Evans

Jónathan Heras

Jonathan Realmuto

Jonathan Resop

Jong Il Rhee

Jong Moon Ha

Jong Yong Lee

Jong Yun Lee

Jongbeom Kim

JongBeom Lim

Jong-Chan Kim

JongCheol Pyo

Jong-Chul Lee

Jong-Ho Shin

Jonghoek Kim

Jong-Hoon Won

Jong-Il Park

Jong-Jin Park

Jongkil Kim

Jongmin Andrew $\mathrm{Yu}$

Jongmin Lee

Jongmin Moon

Jongmin Park

Jong-Myon Kim

Jongpil Jeong

Jong-Ryul Yang

Jongsang Son

Jongshill Lee

Jongsub Moon

Jong-Won Park

Jongwook Jang

Jong-Wook Lee

Jongwoong Park

Jonqlan Lin

Joo Chuan Yeo

Jooeun Ahn
Joohan Kim

Joonas Iivanainen

Joonas Multanen

Joong Ho Shin

Joong-Wook Lee

Joonha Jung

Joonho Seo

Joon-Yong Lee

Joonyoung Kim

Jooyong Kim

Jordi Cusido

Jordi Fonollosa Magrinya

Jordi Mallorqui

Jordi Mongay Batalla

Jordi Ortiz

Jordi Palacín

Jordi Sacristan

Jordi-Roger Riba

Jörg Blankenbach

Jörg Domaschka

Jörg Pezoldt

Jorg Radnik

Jorge Alfredo Ardila-Rey

Jorge Antonio Silva Centeno

Jorge Bernardino

Jorge Brieva

Jorge C. S. Cardoso

Jorge Caceres-Gianni

Jorge Calvillo-Arbizu

Jorge Camacho

Jorge Cerqueiro-Pequeño

Jorge Cesar Masini

Jorge Dias

Jorge E. Ibarra-Esquer

Jorge E. Jimenez-Hornero

Jorge E. Luzuriaga

Jorge Escorihuela

Jorge Fernández-Berni

Jorge Feuchtwanger

Jorge Galván-Tejada

Jorge Garcia-Marquez

Jorge Gosalbez

Jorge Guerra

Jorge Henriques

Jorge Herrera-Tapia

Jorge Igual

Jorge Lanza

Jorge López-Fernández

Jorge Luis Camas Anzueto

Jorge Luis García-Alcaraz

Jorge Luís Victória Barbosa 
Jorge Luis Zambrano Martinez

Jorge Maestre Vidal

Jorge Marcos-Acevedo

Jorge Mario Cruz-Duarte

Jorge Paredes Vieyra

Jorge Pérez-Gómez

Jorge Ricardo Mejía-Salazar

Jorge Ropero

Jorge Sanjurjo-Sánchez

Jorge Tobon

Jorge Torres Sánchez

Joris Pascal

Jorn Mehnen

Jos Elfring

José A. Orosa

José A. Peláez

José A. Rabadán

José A. Siqueira Dias

José Alberto Batista Rodríguez

Jose Alberto Benítez Andrades

José Alberto Gonçalves

Jose Alejandro Galaviz-Aguilar

José Alons Gómez

Jose Angel Corbacho Merino

Jose Antonio Alvarez-Bermejo

Jose Antonio Barrera-Vera

José Antonio De La O Serna

José Antonio Filipe

José Antonio García Naya

Jose Antonio Garcia Souto

Jose Antonio Gazquez Parra

José Antonio González Prieto

Jose Antonio Lozano-Galant

José Azinheira

Jose Balsa-Barreiro

José Benito Bouza-Rodríguez

José Brito

José C. Ramírez-Faz

José Campelo

José Carlos Fernández-García

Jose Carlos Metrôlho

José Cecílio

José Chilo

José Cleiton Sousa Dos Santos

José Cleydson F. Silva

Jose Costa-Requena

José Daniel Hernández Sosa

José Darrozes

Jose De Jesus Agustin Flores Cuautle

Jose De Jesus Rubio

Jose Duenas
José Emilio Guerrero-Ginel

Jose Emilio Meroño

José Fidel Baizabal-Carvallo

José Francisco Gómez-Aguilar

Jose Francisco Saenz-Cogollo

Jose Francisco Tornero-Aguilera

Jose Franco Da Cunha Leme Filho

José Gonçalves

José Guillermo Collí Alfaro

José I. De La Rosa

Jose Ignacio Lijarcio Carcel

Jose Ignacio Priego Quesada

José Ignacio Rodríguez Molano

José Ignacio Serrano

José Israel Martínez-López

Jose Jaime Camacho-Escoto

José Jair Alves Mendes Junior

José Javier Astrain Escola

José Javier Reyes-Lagos

Jose Juan Arranz-Justel

Jose Juan Garcia-Hernandez

Jose L. García-Soidán

José L. Hernández

José L. Pastor

José Lázaro

Jose Luis Cruz-Muñoz

José Luis Díaz-Hernández

José Luis Felipe

José Luis Hernández Ramos

José Luis Hernández-Hernández

José Luis Lima

José Luis López-Martínez

José Luis Olazagoitia

Jose Luis Outón Méndez

Jose Luis Pura

Jose Luis Sanchez-Rojas

Jose Luis Ubago

Jose Luis Valenzuela

Jose Luis Vazquez-Avila

Jose Luiz Sousa Lima

Jose M. Cabeza-Lainez

Jose M. Claver

Jose M. Jimenez

Jose M. Jimenez-Olmedo

José M. Sigarreta

José Machado Da Silva

José Manuel Almerich-Silla

Jose Manuel Andrade

José Manuel Cabeda

Jose Manuel Cano Garcia

Jose Manuel Cano-Garcia 
Jose Manuel De Almeida

José Manuel Díaz-Cruz

Jose Manuel Garcia Alonso

Jose Manuel Gimenez-Guzman

José Manuel M.M. De Almeida

José Manuel Mirás-Avalos

José Manuel Paredes

José Manuel Quero

José Manuel Sáez López

Jose Manuel Soto Hidalgo

José Manuel Villadangos

José Marcato

José Marcato Júnior

José Marcos Ortega

José María Cámara-Zapata

José María Enguita

José María Garrido Balsells

José María López Gullón

José María Martínez-Otzeta

José María Palacios-Santander

Jose Maria Sebastian Y Zuñiga

José María Senciales-González

José Mario De Martino

José Martinez-Carranza

José Martínez-Lillo

José Maurício

José Miguel López-Higuera

José Miguel Mota

Jose Milla

José Naranjo-Torres

Jose Navarro-Pedreño

José Novoa

José Paulo Ferreira Lousado

José Pedro Amaro

José Pelegrí-Sebastiá

Jose Priego-Quesada

Jose R. Guzman-Sepulveda

José R. Llata

Jose R. Valverde

José Raduan Jaber

José Rafael García-Sánchez

Jose Ramírez-Faz

José Ramón Gaxiola-Camacho

José Ramon Lillo-Beviá

Jose Ramón Quevedo Pérez

José Ramón Saura Lacárcel

José Roberto Cardoso

José Rodríguez-Avi

José Saenz

José Saias

José Santa
José Santos

Jose Torres Farinha

José Vicente Francés-Villora

José Vicente Martí

José Vilca

Joseb Blazek

Josef Blažej

Josef Buchner

Josef Pihera

Josefina Gutiérrez

Jose-Maria Serrano Chica

Josep Alos

Josep Lluis Rosa Esteva

Joseph Azar

Joseph Bevitt

Joseph Chang Lun Chan

Joseph Chazalon

Joseph Hupy

Joseph Kvedar

Joseph M. Fortunak

Joseph M. Mahoney

Joseph Nassif

Joseph Ndieyira

Joseph Ng

Joseph P. Hornak

Joseph Snider

Joseph Spernyak

Joseph Verfaillie

Joseph W. Lowdon

Joseph Wasswa

José-Victor Rodríguez

Josh Jessel

Josh Sugar

Joshi Neeraj

Joshua Ensworth

Joshua P. Bailey

Josias Batista

Josip Kasać

Josip Lorincz

Josip Musić

Josip Peranic

Josko Soda

Josko Zec

Jounsup Park

Joydev Ghosh

Joyraj Chakraborty

Joyram Chakraborty

Jože Guna

Jože Kutin

Jože Martin Rožanec

Jozef Gnap 
Jozef Juhár

Józef Lisowski

Józef Ober

Jozef Papan

Jozef Roehrich

Jozsef Katona

Jozue Vieira Filho

Ju Lin

Ju Seok Ryu

Ju Wang

Ju Wook Jang

Juan Antonio Alvarez-Garcia

Juan Antonio Añel

Juan Antonio Castro-Garcia

Juan Antonio Guerrero-Ibáñez

Juan Antonio Holgado-Terriza

Juan Antonio López Ramos

Juan Antonio Martinez Navarro

Juan Antonio Martinez Rojas

Juan Antonio Valera-Calero

Juan Arturo Squella

Juan Bandera

Juan Baselga Llidó

Juan Camilo Vásquez-Correa

Juan Carlos Alvarez Alvarez

Juan Carlos Cuevas Martínez

Juan Carlos Granda

Juan Carlos Martinez Espinosa

Juan Carlos Mosquera

Juan Cheng

Juan Cota-Ruiz

Juan Cruz

Juan De Dios Sanchez-Lopez

Juan F. Prieto

Juan Francisco Dols Ruiz

Juan Francisco Fuentes-Pérez

Juan Gabriel Avina-Cervantes

Juan Gerardo Ávalos

Juan Gregorio Rejas Ayuga

Juan I. Guerrero

Juan Ivan Nieto-Hipolito

Juan J. Castillo

Juan Jesús García Domínguez

Juan Jesús Roldán Gómez

Juan José De Dios

Juan José Escobar

Juan Jose Galiana-Merino

Juan José Jiménez Martín

Juan José López Escobar

Juan José Moyano Campos

Juan José Murillo-Fuentes
Juan Jose Saucedo-Dorantes

Juan José Unzilla

Juan Luis Carús

Juan Luis Gonzalo

Juan Luis Higuera-Trujillo

Juan Luis Navarro-Mesa

Juan Luis Nieves

Juan M Corchado

Juan M Sierra-Hernandez

Juan M. Calderon

Juan M. Gandarias

Juan M. Santos

Juan Manuel Escaño

Juan Manuel Gutiérrez

Juan Manuel Jacinto-Villegas

Juan María Hidalgo-Betanzos

Juan Melchor

Juan Mielgo-Ayuso

Juan Miguel Fernández-Campoy

Juan Miguel Soria Garcia

Juan Mora-Macías

Juan Moreno García-Loygorri

Juan P. Amezquita-Sanchez

Juan P. Domínguez-Morales

Juan P. Vasconez

Juan Pablo Amezquita-Sanchez

Juan Pablo Garcia Vazquez

Juan Pablo Martínez

Juan Pablo Pascual

Juan Pascual-García

Juan Pereiro

Juan Rodrigo Vélez-Cordero

Juan Shan

Juan Villanueva

Juan Yi

Juan Zhu

Juan-Chen Huang

Juan-Luis Gorricho

Juan-Pablo Ramirez-Paredes

Juarez Bento Da Silva

Jucielle Veras Fernandes

Judah Levine

Jude Hemanth

Judicaël Picaut

Jue Hou

Juerg Neuenschwander

Jüergen Bruns

Juha Ala-Laurinaho

Juha Kangasluoma

Juha Roning

Juhani H. Määttä 


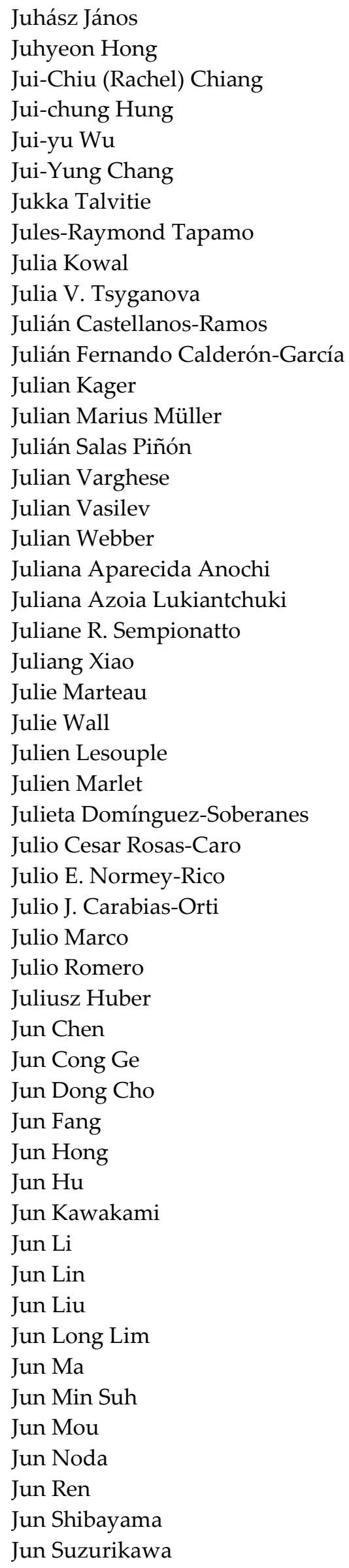

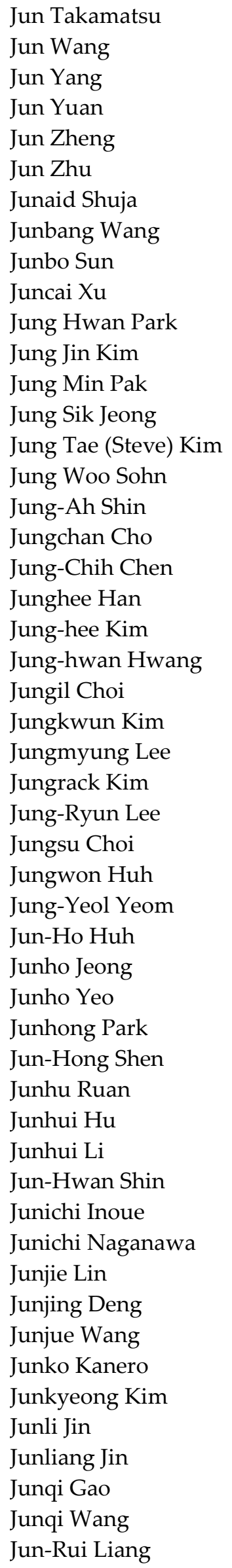




\begin{tabular}{l} 
Junseok Kwon \\
Jun-seok Park \\
Junseop Lee \\
Junsheng Li \\
Junsheng Ren \\
Junsheng Wang \\
Juntong Xi \\
Junwei Cao \\
Jun-Won Ho \\
Junxiang Lian \\
Junxiang Tan \\
Junxiang Zhu \\
Junyi Cao \\
Junzhe Zhang \\
Junzheng Jiang \\
Juraj Bartolic \\
Juraj Dian \\
Juraj Gašinec \\
Juraj Gazda \\
Juraj Gerlici \\
Juraj Kacur \\
Juraj Machaj \\
Juraj Petrović \\
Juraj Sinay \\
Jūratė Sužiedelytė-Visockienė \\
Jürgen Freudenberger \\
Jürgen Hartmann \\
Jürgen Sachs \\
Jurgita Antucheviciene \\
Jurgita Malaiškienė \\
Juri Taborri \\
Juris Burlakovs \\
Justin Albert \\
Justin Chapman \\
Justin F. Schneiderman \\
Kustin Vandenbroucke \\
Justina Sestoke \\
Justus Marquetand \\
Justyna Bohacz \\
Justyna Kozlowska \\
Justyna Libera \\
Justyna Patalas-Maliszewska \\
Justyna Sulej \\
Justyna Tomaszewska \\
Jyh-Miin Lin \\
K. Abhimanyu Kumar Patro \\
Kan \\
Kanit \\
\hline
\end{tabular}

Junseok Kwon

Kah Hou Teng

Kai Chen

Kai Cheng

Kai Ding

Kai Dong

Kai Huang

Kai Liu

Kai Michels

Kai Shen

Kai Wang

Kai Wu

Kai Xiong

Kai Xu

Kai Zhou

Kaichen Song

Kaichen $\mathrm{Xu}$

Kai-Chun Liu

Kaifeng Zhang

Kaige $\mathrm{Wu}$

Kai-Jun Pai

Kaikai Xu

Kailun Yang

Kaimeng Ding

Kaimin Sun

Kaiqing Zhou

Kais Gadhoumi

Kaitao Li

Kaixuan Chen

Kaixuan Zhao

Kaiyuan Shi

Kalathur Santhanam

Kalayci Tuna

Kaleeswaran Balasubramaniam

Kalevi Kilkki

Kalle Tammemae

Kaloyan Yovchev

Kamal Chapagain

Kambiz Tehrani

Kamel Belloulata

Kamil Dimililer

Kamil Gareev

Kamil Kowalczyk

Kamil Krasuski

Kamil Krzywinski

Kamil Makieła

Kamil Staniec

Kamila Haule

Kamila Řasová

Kamran Avanaki

Kamran Iqbal

Kamran Javed 
Kamran Shaukat

Kamrul Islam

Kan Shoji

Kan Wang

Kang An

Kang Ding

Kang Hao Cheong

Kang Shi

Kang Song

Kang-Chun Peng

Kanghyeok Yang

Kangjae Lee

Kangli Liu

Kang-min Lee

Kang-Ming Chang

Kanika Bansal

Kanike Raghavendra Kumar

Kanji Ono

Kaori Fujinami

Kaoru Ichikawa

Kapil Gulati

Kara Gavin

Karcius Day Rosário Assis

Karekin Esmeryan

Karel Charvat

Karel Mls

Karel Pavelka

Karel Roubik

Karen Gordon

Kari Kopra

Kari Lappalainen

Kari V. Vienola

Karim Aly

Karin Anna Hummel

Karin Coninx

Karina Figueroa

Karina Lebel

Karl Andersson

Karl Dietrich Von Ellenrieder

Karla Merazzo

Karla Mossi

Karla Munoz Esquivel

Karlo Griparić

Karna Bryan

Karol Abratkiewicz

Karol Król

Karol Miądlicki

Karol Winkelmann

Karolina Kudelina

Kashif Saleem

Kashif Zia
Kasim Korkmaz

Kaspar Lasn

Kaspar M.B. Jansen

Kasper Van Gasse

Kasper Van Wijk

Kasun Pradeepa Wijayaratna

Katarina Itrić

Katarína Pukanská

Katarzyna Antosz

Katarzyna Bialas

Katarzyna Boratyńska

Katarzyna Harezlak

Katarzyna Jochymczyk-Woźniak

Katarzyna Kopczewska

Katarzyna Kurzatkowska

Katarzyna Marciniak-Łukasiak

Katarzyna Nowakowska-Lipiec

Katarzyna Pentoś

Katarzyna Tyszczuk-Rotko

Katarzyna Zabielska-Adamska

Katarzyna Zawada

Katerina D. Tzimourta

Katerina Kabassi

Katerina Lazarova

Katerina Tzafilkou

Katerine Romeo

Kateryna Sergieieva

Katharina Rombach

Katherine Kirk

Kathiravan Srinivasan

Kathleen A. R. Schildroth

Kathryn Cross

Kathryn L. Havens

Kathyayini Sivasubramanian

Kati Laakso

Katia Pane

Katina Kralevska

Katja Kous

Katrin Schmitt

Katsunori Oyama

Kavita Sharma

Kay-Pong Daniel Yip

Kazimierz Becek

Kazimierz Fabisiak

Kazimierz Gut

Kazimierz Wilkosz

Kazuhiko Kawamoto

Kazuhiro Aruga

Kazuhiro P. Izawa

Kazuki Ikeda

Kazuo Ono 
Kazuo Yonekura

Kazuyuki Nakahata

KC Santosh

Ke Bi

Ke Chen

Ke Feng

Ke Guan

Ke Shi

$\mathrm{Ke} \mathrm{Si}$

Ke Tian

Ke Wang

$\mathrm{Ke} \mathrm{Xu}$

Ke Zhang

Kean C. Aw

Kechen Song

Kee S. Moon

Keekeun Lee

Kefeng Guo

Kefeng Ji

Kegang Zhao

Kei Hang Katie Chan

Kei Masani

Kei Nagashima

Kei Nakagawa

Keiichi Kitajo

Keiji Nagai

Keiko Ogawa

Keisuke Otsuka

Keith Goossen

Keji Yang

Kele Xu

Kelly M. Sullivan

Kelly Sheerin

Kelm Klemens

Kelvin Ian Afrashtehfar

Kelvin Wong

Kemal Sümser

Ken Kiyono

Ken Masamune

Ken Pfeufer

Ken Sasaki

Ken Watanabe

Keng-Hao Liu

Kenichiro Todoroki

Kenji Aono

Kenji Kawashima

Kenji Shimazoe

Kenneth A. Loparo

Kenneth Loh

Kenneth M. Wacha

Kenneth Schepler
Kenneth Sutherland

Kenny Davila

Kenric Nelson

Kenshi Saho

Kensuke Ogawa

Kenta Iitani

Kenta Matsumura

Kenta Obata

Kentaroh Toyoda

Keonwook Kim

Keren Dai

Kerry Peek

Kerry Sun

Kertész István

Keshav Singh

Kesheng Wang

Ketan Kotecha

Ketson Roberto Maximiano Dos Santos

Keunhwa Lee

Keval Vora

Kévin Bouchard

Ke-Vin Chang

Kevin Desai

Kevin F. Bronson

Kevin Huang

Kevin M. Daniels

Kevin Murphy

Kevin Nickels

Kevin Staats

Kevin Tang

Keyan Wang

Keyvan Jaferzadeh

Kezheng Li

Kh Tohidul Islam

Khader Hamdia

Khader Shameer

Khadour Aghiad

Khaldoun Alkhalifeh

Khaled Ardah

Khaled Giasin

Khaled Shuaib

Khalfalla Awedat

Khalid Elgazzar

Khalid Saeed

Khalid Sayood

Khalil Ramadi

Khan Muhammad

Khan Rana Muhammad Asad

Khawaja Khalid Mehmood

Khayyam Masood

Khin Tha 
Khristine Joy C. Antigua

Khurram Hameed

Khushboo Munir

Ki H. Chon

Ki Il Kim

Ki Soo Park

Ki Yong Oh

Kia Dashtipour

Kiang Jean-Fu

Ki-Baek Lee

Kichun Lee

Kien Nguyen

Kien Voon Kong

Kieran Moran

Kifayat Ullah

Kihan Park

Kiho Lim

Ki-Ho Park

Kihwan Kim

Kiichi Niitsu

Ki-Il Kim

Kijin Kim

Kijoon Lee

Kil Chong

Kim Fung Tsang

Kim Jee-In

Kiman Kim

Kimerly Powell

Kimihiro Sakagami

Kimin Yun

Kimmo Keränen

Kin Hing Lo

Ki-Nam Joo

Kinan Ghanem

King Fai Hung

King Man Siu

Kin-hong Wong

Kinjiro Amano

Kira V. Vyatkina

Kiril Alexiev

Kirill Tomyshev

Kishor Datta Gupta

Kishu Ranjan

Ki-Sun Lee

Kittisak Jermsittiparsert

Kiwon Moon

Kiyotaka Fujisaki

Kiyoung Kim

Kjartan Halvorsen

Klaas Dijkstra

Klaas Pauly
Klara Megyeri

Klas Ihme

Klaske Van Kammen

Klaudia Smoląg

Klaus Coco

Klaus Koren

Klaus Mathwig

Klaus Michael Spohr

Klaus Schäfer

Klaus Szielasko

Klaus Thoeni

Klaus Werner Stöckelhuber

Kleftodimos Alexandros

Klemen Bučar

Klemen Kregar

Kleomenis Kalogeropoulos

Knut Øvsthus

Kogularasu Sakthivel

Koichi Kurita

Koichi Maezawa

Koichiro Miyamoto

Koichiro Shiomori

Koji Hashiguchi

Koji Inoue

Kongzhe Chen

Konlin Shen

Konrad Jerzy Kapcia

Konrad Kielbasinski

Konrad Mollenhauer

Konrad Rudnicki

Konrad Waluś

Konstantin B. Yushkov

Konstantin E. Samouylov

Konstantin Gugleta

Konstantin Kozlov

Konstantin Markov

Konstantin Mikhaylov

Konstantin Mikhelson

Konstantin Neusypin

Konstantin Nikolaev

Konstantin P. Andryushin

Konstantin P. Gaikovich

Konstantin Rumyantsev

Konstantinos A. Banitsas

Konstantinos C. Zikidis

Konstantinos Demertzis

Konstantinos Giannakis

Konstantinos Karampidis

Konstantinos Kolias

Konstantinos Kotis

Konstantinos Limniotis 
Konstantinos M. Giannoutakis

Konstantinos Makantasis

Konstantinos Maliatsos

Konstantinos Misiakos

Konstantinos Nikolakopoulos

Konstantinos Tountas

Konstantinos Tsongas

Konstantins Jefimovs

Koojana Kuladinithi

Kooktae Lee

Koorosh Gharehbaghi

Körstgens Volker

Kosai Raoof

Kosmas Alexopoulos

Kosmas Dimitropoulos

Kosmas Kavadias

Kostas Kolomvatsos

Kostas Peppas

Kostas Yiannopoulos

Kota Kodama

Kothandam Gopalakrishnan

Kouichi Sakurai

Kourosh Zareinia

Kovacic Bostjan

Kranthi K. Maniam

Krassimira Vlachkova

Krešimir Trontl

Krishanu Roy

Krishna P. Kadiyala

Krishna Persaud

Krishnamurthy Vemuru

Krishnan Murugappan

Kristel Fobelets

Kristen M. Meiburger

Kristi Santi

Kristian Dokic

Kristian Kostal

Kristian Mathis

Kristijan Lenac

Kristina Daunoraviciene

Kristina L. Allen-Brady

Kristína Machová

Kristina Stojmenova

Kristina Šutienė

Kristine Krajnak

Kristoffer Petersson

Krystian Erwiński

Krystian Jobczyk

Krystian Kozioł

Krystian Mistewicz

Krystian Pyka
Krystian Radlak

Krystyna Jabłońska

Krzysztof B. Beć

Krzysztof Bartecki

Krzysztof Blecharz

Krzysztof Brzostowski

Krzysztof Chwastek

Krzysztof Czaplewski

Krzysztof Dołowy

Krzysztof Gaska

Krzysztof Górecki

Krzysztof Górnicki

Krzysztof Grochla

Krzysztof Jamroziak

Krzysztof K. Dudek

Krzysztof Karwowski

Krzysztof Klimaszewski

Krzysztof Kluza

Krzysztof Kukielka

Krzysztof Kulpa

Krzysztof Małecki

Krzysztof Musioł

Krzysztof Naus

Krzysztof Nyka

Krzysztof Okarma

Krzysztof Pielichowski

Krzysztof Pieńkowski

Krzysztof Przybył

Krzysztof Przystupa

Krzysztof Psiuk-Maksymowicz

Krzysztof R. Karsznia

Krzysztof Rechowicz

Krzysztof Rogowski

Krzysztof Ryczko

Krzysztof Rzecki

Krzysztof Sawicki

Krzysztof Schabowicz

Krzysztof Skabek

Krzysztof Skrzypkowski

Krzysztof Sośnica

Krzysztof Stępień

Krzysztof Szabat

Krzysztof Tadyszak

Krzysztof Targiel

Krzysztof Tomczyk

Krzysztof Wajda

Krzysztof Walas

Krzysztof Wałęsa

Krzysztof Wesolowski

Ksenia Ostrowska

Kshitij Sharma 
Kuan Sun

Kuang Sheng

Kuang-Hao Lin

Kuan-Hui Lee

Kuanyu Chen

Kuen-Suan Chen

Kui Xu

Kumar Biswajit Debnath

Kumar Prashant

Kumar Yelamarthi

Kun Jia

Kun Lin Lee

Kun Liu

Kun Qian

Kun Xu

Kun Zhang

Kun-Chih Chen

Kun-Hui Chen

Kuniaki Nagamine

Kunjie Chen

Kun-mean Hou

Kunpeng Feng

Kuo-Chang Lu

Kuo-Cheng Huang

Kuo-Chih Chuang

Kuo-Hu Chen

Kuo-Jen Chang

Kuo-Kun Tseng

Kuo-Liang Chung

Kuo-Liang Huang

Kuo-Lung Lian

Kuo-Lung Wang

Kuo-Ping Lin

Kuo-Yi Lin

Kurt Ammer

Kurt Debattista

Kurt Maly

Kurt W. Kolasinski

Kutubuddin Ansari

Kwan Kyu Park

Kwang Lee

Kwang Ryul Baek

Kwang-Hoon Kim

Kwangseok Oh

Kwok Ping Chan

Kwok Tai Chui

Kwok-Fan Chow

Kwo-Ting Fang

Kyandoghere Kyamakya

Kyeong-Keun Choi

Kyle Nelson
Kyle R. Wilt

Kyo Inoue

Kyong Hon Kim

Kyoung Youl Park

Kyriaki Kostoglou

Kyu Tae Park

Kyu-haeng Lee

Kyujung Kim

Kyuman Lee

Kyung Chul Oh

Kyung Hyun Choi

Kyung Wha Oh

Kyungeun Sung

Kyungha Min

KyungHi Chang

Kyung-Joon Park

Kyungkwang Joo

Ladislav Beranek

Ladislav Főző

Ladislav Mariš

Ladislav Polak

Laeticia Petit

Laia Subirats

Laibing Jia

Lai-Kwan Chau

Laio O. Seman

Laio Oriel Seman

Laís Canniatti Brazaca

Laith Alzubaidi

Lajos Daróczi

Lal Mohan Bhowmik

Laleh Avazpour

Laleh Badriasl

Lalitha Madhavi Konila Sriram

Lambros Ekonomou

Lamiaa Elrefaei

Lan Xiang

Lan Zhang

Lana Ruzic

Lance Champagne

Lang Xu

Lanto Rasolofondraibe

Lapo Miccinesi

Lara A. Thompson

Lara Del Val

Lara Motta

Larisa Dunai

Larisa Lvova

Larissa Pereira Ribeiro Teodoro

Lars Ohlsson Fhager

Larysa Neduzha 


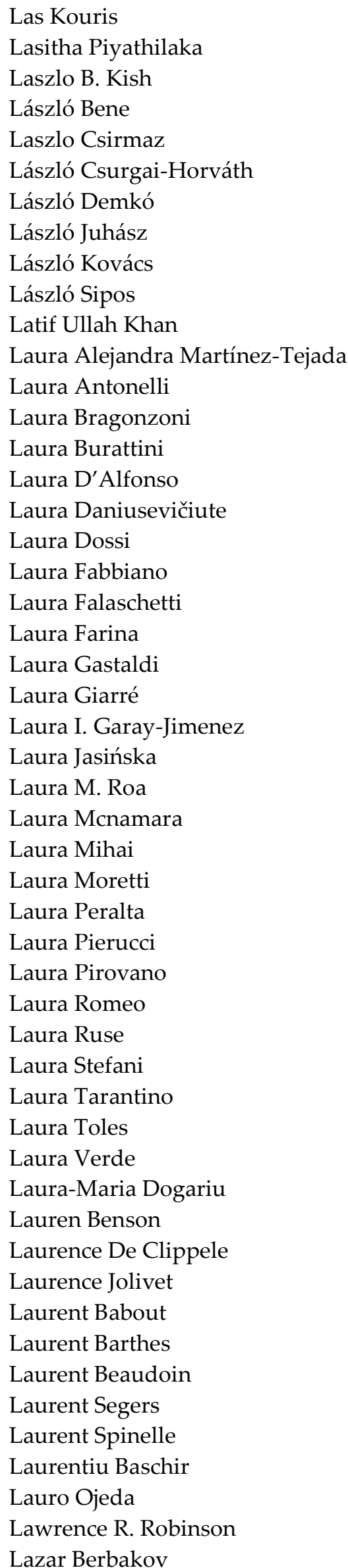

Lázaro Eduardo Da Silva

Lazaros Aresti

Lazo M. Manojlović

Lazzaro Di Biase

Le Sun

Le Van Minh

Le Yao

Leandro Buss Becker

Leandro Maio

Lefteris Benos

Lehel Csató

Lei Fan

Lei Gao

Lei Guo

Lei Han

Lei Huang

Lei Jing

Lei Li

Lei Mao

Lei Mei

Lei Qiu

Lei Shi

Lei Wan

Lei Wang

Lei $W u$

Lei Xue

Lei Yu

Lei Zhai

Lei Zhang

Lei Zhou

Lei Zhu

Leijiao Ge

Leila Keshavarz

Leila Merghem Boulahia

Leilei Li

Lei-Ming Ma

Lenka Lhotska

Lenny Van Erp-van Der Kooij

Leo K. Cheng

Léo Martire

Leobardo Hernandez

Leocundo Aguilar Noriega

Leon Reznik

Leon Rothkrantz

Leon Stefanija

Leonard Daus

Leonard Felicetti

Leonard Hanssen

Leonard Stoica

Leonardo Acho Zuppa

Leonardo Aguayo 
Leonardo Alexandre Peyré-Tartaruga

Leonardo Angelini

Leonardo Ciaccheri

Leonardo D' Acquisto

Leonardo Franco

Leonardo Garcia-Garcia

Leonardo Honório

Leonardo Juan Ramirez Lopez

Leonardo Nascimento Ferreira

Leonardo Pantoli

Leonardo Rundo

Leonardo Vicarelli

Leoneed M. Kirilov

Leonel Jorge Ribeiro Nunes

Leonel Paredes-Madrid

Leong Chuan Kwek

Leonid Bolotov

Leonid Fetisov

Leonid Satrapinskyy

Leonid V. Katkovsky

Leonidas Kouris

Leopoldo Acosta Sanchez

Leopoldo Altamirano Robles

Lesley Gibson

Leslie Ching Ow Tiong

Leslie Wong

Lesya Anishchenko

Leszek Ambroziak

Leszek Luchowski

Leszek Nowosielski

Letitia Mirea

Lev A. Matveev

Lev Jakub

Lev Kuzmin

Lev T. Perelman

Levente Czumbil

Levente Klein

Levente Tamás

Lewei Tang

Lewis Nkenyereye

Leyuan Liu

$\mathrm{Li} \mathrm{Fu}$

Li Gang

Li Kun

$\mathrm{Li} \mathrm{Li}$

Li Lin

Li Liu

Li Sun

Li Tan

Li Yang

Li Zhao
Li Zheng

Lia E Aciu

Lian Yu Zheng

Liana Stanescu

Lianbo Ma

Liandong Li

Lianfu Han

Liang Chen

Li-Ang Lee

Liang Liang

Liang Xiao

Liang Xie

Liang Zhao

Liang Zou

Liang-Bi Chen

Lianggui Liu

Liang-Hung Wang

Liangjiang $\mathrm{Yu}$

Liangjing Yang

Liangliang Cheng

Liangliang Yang

Liangtian Wan

Liangxing $\mathrm{Hu}$

Lianjie Zhou

Lianming Li

Liansheng Wang

Lianwu Guan

Lianyong Qi

Libin Huang

Libin Ye

Libo Gao

Libo Ma

Libo Meng

Libo Zhao

Libor Hargas

Libor Pekar

Libor Váša

Lichota Piotr

Li-Chung $\mathrm{Wu}$

Lidia Fiorini

Lidia Fotia

Lidia Ogiela

Lidia Sánchez González

Lidong Yang

Lieselotte Corten

Lieva Van Langenhove

Lifei Wang

Li-Fong Lin

Lifton Joseph John

Liguo Sun

Lihai Zhang 
LiHaojun Li

Lihong Duan

Lihua Li

Lihua Ma

Lihua Wang

Lihui Feng

Lihui Luo

Lijun Sun

Lijun Zhang

Lijun Zhao

Lili Wan

Lili Xing

Lilian Sosa

Lilian Witthauer

Liliana Fadul-Pacheco

Liliana Porojan

Liliana Szyszka-Sommerfeld

Liliana Vale Costa

Lilly Li

Liming Fang

Liming Nie

Liming Wang

Lin Cao

Lin Chen

Lin Feng

Lin Lin

Lin Meng

Lin Qi

Lin Shu

Lin Wang

Lin $\mathrm{Wu}$

Lin Zhang

Lina Garcés

Lina Lundgren

Lina Mohjazi

Lina Zou

Linas Petkevičius

Linas Svilainis

Linbo Shao

Linchao Li

Lincoln Silva

Linda M. Eerikäinen

Linda PaternÒ

Linda Senigagliesi

Lindsay Bottoms

Lindy Blackburn

Linfeng Xu

Ling Chen

Ling Tim Wong

Ling $\mathrm{Yu}$

Lingfei Mo
Lingfu Kong

Lingjia Gu

Lingju Meng

Lingli $\mathrm{Yu}$

Lingling Ma

Lingqian Zhang

Lingwei $\mathrm{Xu}$

Lingxia $\mathrm{Mu}$

Lingxiang Zheng

Lingxin Chen

Ling-Yun Li

Lingzhong Guo

Linh Nguyen

Linh Truong-Hong

Linhui Sun

Linhui Zhao

Linling Kuang

Linmi Tao

Lino Figueiredo

Linpei Li

Linping Zhao

Linqing Luo

Linru Xu

Linyi Liu

Linyuan Xia

Linyue Gao

Lionel Nkenyereye

Lionel Reveret

Lior Medina

Lipan Leontina

Liping Du

Liping Huang

Liping Qi

Li-Ping Xu

Liqiang Li

Liqiang Nie

Liqin Cao

Liqin Ge

Liqing Chen

Liqun Hou

Liqun Sun

Lis K. Nanver

Lisa M. Gallagher

Lisandro Lovisolo

Liseane Padilha Thives

Lisheng Fan

Lisheng Jin

Lisheng Xu

Lishengsa Yue

Lisu Yu

Litao Han 
Liu Wang

LiuDi Jiang

Liudmila G. Shamanaeva

Liudmila I. Gerasimova-Meigal

Liutauras Marcinauskas

Liuyang Zhang

Liuyi Ling

Live Steinnes Luteberget

Livia Alexandra Dinu Gugoasa

Lívia Florio Sgobbi

Livia Petrescu

Livinti Petru

Livio D'Alvia

Liviu C. Miclea

Liviu Ciupitu

Liviu Duta

Liviu Viman

Liviu-Adrian Cotfas

Li-Wei Kang

Li-Wei Ko

Liwei Li

Liwei Liu

Lixiang Li

Lixiang $\mathrm{Wu}$

Liyan Zhang

Liyaning (Maggie) Tang

Liza Lee

Lizeth Torres

Lizhen Wang

Lizhi Pan

Ljubomir Vračar

Llinbo Li

Llorenç Cerdà-Alabern

Lluís Albarracín

Lo Tiantian

Logan T. Trujillo

Loïca Avanthey

Loizos Pelecanos

Lok Shrestha

Lonesome Malambo

Long Chiau Ming

Long Kang

Long $\mathrm{Li}$

Long Lingliang

Long Liu

Long Qi

Long Que

Long T. Truong

Long Zhang

Longjiang $\mathrm{Li}$

Longju Liu
Longjun Dong

LongKun Guo

Longsheng $\mathrm{Fu}$

Longteng Tang

Longxiang Guo

Loredana Dumitrascu

Lorella Marinucci

Lorena Parra

Lorena Parra Boronat

Lorenzo Angeletti

Lorenzo Brognara

Lorenzo Carnevale

Lorenzo De Carli

Lorenzo Faggioni

Lorenzo Grazi

Lorenzo Iorio

Lorenzo Lotti

Lorenzo Mucchi

Lorenzo Olivieri

Lorenzo Ortega

Lorenzo Palma

Lorenzo Putzu

Lorenzo Ricci

Lorenzo Ricciardi Celsi

Lorenzo Rosa

Lorenzo Scalera

Lorenzo Spinelli

Lorenzo Teppati Losè

Lorenzo Vangelista

Loreto Di Donato

Loreto Pescosolido

Lori-Ann R. Sacrey

Loris Nanni

Loris Roveda

Lotfi Chaari

Louise Ada

Louise Craig

Louise Devigne

Loukas Bampis

Lounis Chermak

Lourdes María Fernández Seguín

Lourenco Pereira

Lovorka Libric

Lu Bai

Lu Guo

$\mathrm{Lu} \mathrm{Yu}$

Lu Zhang

Lua Ngo

Luana Lugini

Luana Olivieri

Lubos Buzna 
L'uboš Ovseník

Lubos Rejfek

Luboš Smolík

Luca Abeni

Luca Ardigò

Luca Baradello

Luca Bruschini

Luca Bruzzone

Luca Calderoni

Luca Cicala

Luca Cosmo

Luca D'Acierno

Luca Davoli

Luca De Stefano

Luca De Vito

Luca Di Angelo

Luca Ferretti

Luca Fredianelli

Luca Labate

Luca Lombardi

Luca Magistrelli

Luca Martino

Luca Massidda

Luca Oggioni

Luca Pallotta

Luca Paolo Ardigò

Luca Piancastelli

Luca Piroddi

Luca Reggiani

Luca Rosafalco

Luca Sani

Luca Santini

Luca Schirru

Luca Testarelli

Luca Vollero

Lucas Blandón-Naranjo

Lucas De Oliveira Teixeira

Lucas Encarnação

Lucas F. Gerez

Lucas J. Koerner

Lucas Prado Osco

Lucas V. R. Alves

Luci Martin

Lucia Billeci

Lucia Hilario Pérez

Lucia Nardone

Lucia Rusu

Lucian Gheorghe Gruionu

Lucian Petrescu

Lucian Stefanita Grigore

Lucian Trifina
Luciana Freitas Bastos

Luciana Labanca

Luciane Silva Martello

Lucian-Mihai Cosovanu

Luciano Alves De Oliveira

Luciano Bertini

Luciano Boesel

Luciano Nocera

Luciano Scaltrito

Lucija Brezočnik

Lucile Rutkowski

Lucinda Leonard

Lucja Dybowska-Sarapuk

Lucjan Jerzykiewicz

Lucjan Kozielski

Lucjan Setlak

Ludovico Valli

Ludovit Kovanic

Lu-Hua Fu

Luige Vladareanu

Luigi Benedicenti

Luigi Capozzoli

Luigi Coppolino

Luigi De Bellis

Luigi De Napoli

Luigi De Simio

Luigi Di Costanzo

Luigi Fortuna

Luigi La Spada

Luigi Manfredi

Luigi Maria Galantucci

Luigi Meccariello

Luigi Vertuccio

Luigi Vito Stefanelli

Luis Abegão

Luis Andreu Caravaca

Luis Anido

Luis Barba-Guaman

Luís Bernardo

Luís Carlos Santos

Luis Carús

Luis Coelho

Luís Correia

Luis Cruz-Piris

Luis Díaz-Ballote

Luis Emanuel Moutinho Silva

Luis Enrique García-Muñoz

Luis Enrique González Jiménez

Luis Fernando De Mingo López

Luis Fernando Herrán

Luis Fernando Luque Vega 
Luis Francisco Díez

Luis G. De La Fraga

Luis Gómez Déniz

Luis Gonzaga Baca Ruiz

Luis Hernandez

Luis Hernández-Callejo

Luis Iglesias

Luis Ignacio Lopera González

Luis J. De La Cruz Llopis

Luis Jesús Villarreal-Gómez

Luis Jorda-Bordehore

Luis M. Camarinha-Matos

Luis Miguel Gomes Abegão

Luis Moutinho

Luis Moyne

Luis Muñoz-Saavedra

Luis N. López De Lacalle

Luis Naranjo-Zeledón

Luis Omar Colombo-Mendoza

Luis Orozco Barbosa

Luis Parrilla

Luis Parrilla Roure

Luis Payá

Luis Pérez-Domínguez

Luís Pinto Da Silva

Luís Proença

Luis Rodriguez-Benitez

Luís Rosa

Luís Roseiro

Luis Sanabria-Russo

Luis Urquiza-Aguiar

Luis Velasco

Luisa Gomes Pereira

Luisa Jorge

Luisa Quevedo

Luiz Antonio Baccala

Luiz Carlos Sandoval Goes

Luiz Eduardo Virgilio Da Silva

Luiz Gonçalves

Luka Pavic

Luka Rumora

Luka Vukić

Lukas Chrpa

Lukas Chruszczyk

Lukáš Kučera

Lukas Margaritis

Lukáš Ondic ${ }^{\longleftarrow}$

Lukáš Peter

Lukáš Richtera

Lukasz Ambrozinski

Łukasz Bednarz
Łukasz Dziuda

Łukasz Jankowski

Lukasz Janowski

Łukasz Januszkiewicz

Lukasz Jelen

Łukasz Knypiński

Łukasz Krzywiecki

Łukasz Kulas

Lukasz Makowski

Łukasz Maliński

Łukasz Maślikowski

Łukasz Oleksy

Lukasz Orman

Lukasz Sadowski

Łukasz Sienkiewicz

Lukasz Sójka

Łukasz Szeleszczuk

Lukasz Wieclaw

Łukasz Wyciślik

Lulu Sun

Lulu Wang

Luminita Ghervase

Luminița Moraru

Lun-Chi Chen

Lunke Fei

Luo Liu

Lurui Zhao

Luz Berenice López-Hernández

Luz Del Carmen Gómez-Pavón

Luzheng Bi

Lvwen Huang

Lydia Min-Ying Su

Lydia Sobotova

Lynda Sedikki

Lyubka Pashova

Lyudmila Dimitrova

M. A. Hannan Bin Azhar

M. Ariel Wallace

M. Gomez-Gonzalez

M. Mahmood Ali

M. Mustafa Rafique

M. Pilar Garde

M. Salauddin Rasel

M. Shamim Kaiser

M. Teresa Teixidó Ullod

M. Z. Naser

M.S. Zobaer

Mํㅗ Nieves Piña Capó

Maanak Gupta

Maarten Weyn

Macedon Moldovan 


\author{
Maciej Gucma \\ Maciej Haras \\ Maciej Hojda \\ Maciej Jedliński \\ Maciej Klaczynski \\ Maciej Kuboń \\ Maciej Majewski \\ Maciej Matuszewski \\ Maciej Matys \\ Maciej Nikodem \\ Maciej Nowak \\ Maciej Romaniuk \\ Maciej S. Wróbel \\ Maciej Stefańczyk \\ Maciej Sułowicz \\ Maciej Trusiak \\ Maciej Wojtczak \\ Maciej Zaborowicz \\ Maciej Zajkowski \\ Madaín Pérez Patricio \\ Madalina Dumitriu \\ Madalina Simona Baltatu \\ Madalina-Georgiana Berceanu \\ Mădălin-Dorin Pop \\ Madallah Alruwaili \\ Madhu Gyawali \\ Madhu Sheri \\ Madis Ratassepp \\ Maeda Hiroaki \\ Magdalena Czarnogorska \\ Magdalena Igras-Cybulska \\ Magdalena Kachniewska \\ Magdalena Kopernik \\ Magdalena Król-Zielińska \\ Magdalena Kusnierz \\ Magdalena Machno \\ Magdalena Mieloszyk \\ Magdalena Niemczewska-Wójcik \\ Magdalena Palacz \\ Magdalena Pilarska \\ Magdalena Stobiecka \\ Magdalena Szymczyk \\ Magdalena Valentina Lungu \\ Magdalena Włodarska \\ Maged Mahmoud Marghany \\ Maggie He \\ Magna Gabriele \\ Magnus Holm \\ Magnus Westerlund \\ Mahdi Heydari \\ Mahdi Naghshvarianjahromi
}

Mahdi Rezapour

Mahdi Takaffoli

Mahdi Yousefi

Mahdi Zareei

Mahendra Piraveenan

Mahendra Shukla

Maher Khaliel

Mahesh Soni

Mahima Agumbe Suresh

Mahmood Shafiee

Mahmoud Elbattah

Mahmoud Elsisi

Mahmoud Hassan

Mahmoud Nabil

Mahmoud Nasr

Mahmoud Ouda

Mahmoud R. M. Atalla

Mahmoud Wagih

Mahyar Khorasani

Mai The Vu

Maik Herbig

Maiko Sakamoto

Mailyn Pérez Liva

Maira Beatriz Hernandez Moran

Maira Martins Da Silva

Maja Bryk

Maja Goršič

Maja Krčum

Maja Matetic

Maja Pusnik

Majeed Abdul

Majid Hosseinpour

Majid Khazaee

Majid Mufaqam Syed-Abdul

Maju Kuriakose

Makhsudsho G. Nematov

Makiko Kobayashi

Makoto Aoki

Makoto Hasegawa

Makoto Miyakoshi

Malak Henchiri

Malcolm Joyce

Malgorzata Domino

Małgorzata I. Michalczyk

Malgorzata Klaudia Guzowska

Małgorzata Norek

Małgorzata Ochota

Malgorzata Orczyk

Malgorzata Pankowska

Małgorzata Plechawska-Wójcik

Malgorzata Renigier-Bilozor 
Malgorzata Syczewska

Małgorzata Sztubecka

Małgorzata Wojtaszek

Malick Diakhate

Malik Haddad

Malik M. Naeem Mannan

Malinka Ivanova

Malte Frovel

Malte Misol

Mamede De Carvalho

Mamoona Humayun

Mamta Agiwal

Mamtha Balla

Manabu Ataka

Manabu Sato

Manabu Tsukada

Manasvi Lingam

Manel Del Valle

Manel Taboada

Manish Bhatt

Manisha Sahu

Manlio Bacco

Manmeet Mahinderjit Singh

Manob Jyoti Saikia

Manod Williams

Manohar Das

Manohar Prasad Bhandari

Manoj Kumar Mahata

Manoj Panda

Manolis Tsiknakis

Manos Panaousis

Mansur As

Manuel A. Armada

Manuel Aleixandre

Manuel Arbelo

Manuel Arias-Montiel

Manuel Arrebola

Manuel Au-Yong-Oliveira

Manuel Bou-cabo

Manuel Buitrago

Manuel Cabaleiro

Manuel De La Torre Juarez

Manuel Eugenio Morocho-Cayamcela

Manuel F. González-Penedo

Manuel Fernández-Veiga

Manuel Ferre

Manuel Filipe Pereira Cunha Martins Costa Marc Weinberg

Manuel Gabler

Manuel García García

Manuel García Sánchez

Manuel Graña

Marc Wittmann
Manuel Günther

Manuel Gutiérrez-Capitán

Manuel Herrera

Manuel J. C. S. Reis

Manuel J. Fonseca

Manuel Jesús Domínguez-Morales

Manuel Joaquim Bastos Marques

Manuel Joaquim Sabença Feliciano

Manuel Lopez-Martin

Manuel Martinez

Manuel Moreno

Manuel Ocaña

Manuel Omar Meranza-Castillón

Manuel Perea

Manuel Prado-Velasco

Manuel Rodriguez-Martin

Manuel Rodriguez-Rastrero

Manuel Sierra-Castañer

Manuel Utrilla-Manso

Manuel V. Ramallo

Manuel Vargas Villanueva

Manuela Avadanei

Manuela Chessa

Manuela Ingaldi

Manuela Zude

Mao Mao

Mao Shan

Maohua Lin

Maohua Pan

Maokun Li

Maolin Chen

Maomao Chen

Maorong Ge

Maosen Wang

Maosi Chen

Mar Vilanova De La Torre

Maram Bani Younes

Marat Mukhametzhanov

Marc Brecht

Marc Duquennoy

Marc Frincu

Marc Kurz

Marc Marín-Genescà

Marc Mateu-Mateus

Marc Solal

Marc Vidal

Marcel Ameloot

Marcel Bouvet

Marcel Fajkus 
Marcel Kordoš

Marcel Kuruc

Marcel Nicola

Marcela Alina Fărcașiu

Marcela Bindzárová Gergel’ová

Marcela Munera

Marcello Berto

Marcello Costa

Marcello Di Risio

Marcello Iasiello

Marcello Magri Amaral

Marcello Sanguineti

Marcelo Bender Perotoni

Marcelo David

Marcelo Faria

Marcelo Félix Alonso

Marcelo Fernandes

Marcelo García

Marcelo Gitirana

Marcelo Hazin Alencar

Marcelo Nogueira

Marcelo Petry

Marcelo Sampaio De Alencar

Marcelo Werneck

Márcia Barros

Márcia C. Figueiredo

Marcin Adamczyk

Marcin Balcerzyk

Marcin Bernaś

Marcin Chodźko

Marcin Ciecholewski

Marcin Derlatka

Marcin Derwich

Marcin Drozd

Marcin Gnyba

Marcin Górski

Marcin Habrych

Marcin Jagoda

Marcin Jesionek

Marcin Kafarski

Marcin Kicinski

Marcin Kluczyk

Marcin Kolakowski

Marcin Korzeń

Marcin Kowalski

Marcin Kremieniewski

Marcin Lackowski

Marcin Lawnik

Marcin Luckner

Marcin Nabiałek

Marcin Piekarczyk
Marcin Pluciński

Marcin Rudzki

Marcin Spiralski

Marcin Staniek

Marcin Straczkiewicz

Marcin Suszynski

Marcin Urbanowicz

Marcin Watorek

Marcin Witczak

Marcin Wozniak

Marcin Zych

Márcio Falcão Santos Barroso

Márcio Júnior Lacerda

Marcius Fabius De Carvalho

Marco A. Moreno-Armendáriz

Marco A. Panduro Panduro

Marco Abbatangelo

Marco Alexandre Ribeiro

Marco Antonio Álvarez González

Marco Antonio Perez

Marco Antonio Sotelo Monge

Marco Antonio Teixeira

Marco Arnesano

Marco Aurelio Nuño-Maganda

Marco Balsi

Marco Baù

Marco Bernardo

Marco Bindi

Marco Bobinger

Marco Bonopera

Marco Botta

Marco Bravi

Marco Buiatti

Marco Buzzelli

Marco Caniato

Marco Capogni

Marco Cardenas-Juarez

Marco Carminati

Marco Carotenuto

Marco Carratù

Marco Cassinelli

Marco Ceccarelli

Marco Cicala

Marco Ciccone

Marco Civera

Marco Claudio De Simone

Marco Cocconcelli

Marco Costanzo

Marco Crescentini

Marco D. Vásconez-Maza

Marco D'Alessandro 
Marco D'Alonzo

Marco Dell'Isola

Marco Depiante

Marco Esposito

Marco Faggella

Marco Filippucci

Marco Furinghetti

Marco Gabiccini

Marco Gadola

Marco Gazzoni

Marco Germanotta

Marco Gervasi

Marco Ghislieri

Marco Grossi

Marco Iosa

Marco Knaflitz

Marco Lapegna

Marco Laracca

Marco Laurino

Marco Leo

Marco Limongiello

Marco Listanti

Marco Lombardi

Marco Maggiali

Marco Mandolini

Marco Marcon

Marco Martalo

Marco Matassoni

Marco Mauri

Marco Mercuri

Marco Miliucci

Marco Miniaci

Marco Mobilio

Marco P. Soares Dos Santos

Marco Pérez-Hernández

Marco Piccirilli

Marco Picone

Marco Porta

Marco Portelli

Marco Reggiannini

Marco Riani

Marco Righi

Marco Roberto Cavallari

Marco Rosone

Marco Sampietro

Marco Sanna Angotzi

Marco Santonico

Marco Scharringhausen

Marco Schiavone

Marco Stefano Demarchi

Marco Tallini
Marco Tregua

Marco Vaccari

Marco Vinicio Hernández Arriaga

Marco Zappatore

Marcos Arza

Marcos Benedito Schimalski

Marcos Cavenaghi

Marcos De Oliveira Junior

Marcos Díaz Quezada

Marcos Eduardo Valle

Marcos F. S. Teixeira

Marcos Quiñones-Grueiro

Marcos Rodrigues

Marcos Schimalski

Marcos Silva Martins

Marcos Tostado-Véliz

Marcus Hammer

Marcus OW Grimm

Marcus Schmidt

Marcus Tullius Scotti

Marcus Varanis

Marcus Vollmer

Marderos Sayegh

Mare Koit

Marek Amanowicz

Marek Barski

Marek Bolanowski

Marek Bugaj

Marek Długosz

Marek Fraštia

Marek Gancarz

Marek Jaskiewicz

Marek Konefał

Marek Kozień

Marek Kraft

Marek Krawczuk

Marek Lampart

Marek Lefik

Marek Magdziak

Marek Miskowicz

Marek Moravcik

Marek Penhaker

Marek Pokorny

Marek Prochazka

Marek Przyborski

Marek Sawicki

Marek Sikora

Marek Słoński

Marek Stencel

Marek Szlósarczyk

Marek Urbanik 
Marek Vagaš

Marek Węglowski

Marek Wesołowski

Marek Życzkowski

Marek Zygmunt

Maren Friesen

Margarita Tecpoyotl-Torres

Margarita-Arimatea Diaz Cortes

Margherita Capriotti

Margherita Peruzzini

Maria Adele Giamberardino

Maria Alexandra Teodósio

Maria Angeles Caballero-Mora

María Ángeles Verdejo Espinosa

Maria Antonietta Ferrara

Maria António Castro

Maria Baldeon Calisto

María Blanca Caminero

Maria Bostenaru

Maria Butakova

Maria C.T. Cangussú

María Campo-Valera

María Carmen Blanco-López

María Carmen Carnero

María Carmen Pérez

Maria Colomba Comes

María Concepción Pérez Cárceles

María Criado Sanz

Maria D. Miranda

Maria Da Graca Campos Pimentel

Maria De Lourdes Bueno Trindade Galo

Maria Del Carmen Romero-García

María Dolores Fernández Ramos

María Dolores Gómez Pulido

Maria Eduarda Ferreira

Maria Elisa Tata

Maria Erminia Genovese

María Estrella Sousa Vieira

Maria Fátima Paulino

Maria Francesca Piacentini

María Francisca Carreño

María Francisca Rosique Contreras

Maria Gabriela Lagorio

Maria Gabriella Xibilia

Maria Gamella Carballo

María García Fernández

Maria Giovanna Bianco

Maria Giovanna Masciotta

Maria Giuseppina Limongelli

Maria Graça Ruano

Maria Grazia Grimaldi
Maria Grazia Manera

Maria Gritsevich

María Isabel Prieto Barrio

María Isabel Rodriguez Ferradas

Maria Jesus De La Fuente Aparicio

María Jesús Lobo-Castañón

María José García-Pola

Maria Jose Gomez-Silva

Maria K. Rybarczyk

Maria Karatassiou

Maria Kihl

Maria Konstantaki

Maria Korozi

Maria Krechowicz

Maria Lucia Miglietta

Maria Luisa Braunger

Maria Luisa Villani

Maria Luz Rodriguez-Mendez

Maria Maddalena Calabretta

Maria Magdalena Bujnowska-Fedak

Maria Matsiola

Maria Mendoza-Muñoz

Maria Mrówczyńska

María Napal

Maria Nisheva

Maria Panitsa

Maria Pedrero

Maria Pia Viggiano

María Pilar Pecci Lloret

Maria Pop

Maria R Bonsignore

María R. Fernández-Ruiz

Maria Ramos Payán

Maria Raposo

Maria Rashidi

Maria Rigou

Maria Rizzi

Maria Romano

Maria Rubega

Maria Seimeni

Maria Selezneva

Maria Sergeeva

Maria Sileo

Maria Strianese

Maria Surmeneva

Maria Teresa Baeza-Romero

María Teresa García-Ordás

María Teresa Lázaro

María Teresa López

María Teresa Sanz-Pascual

Maria Vasconcelos 
Maria Vesna Nikolic

María Victoria Sebastián

María Yolanda Castellote-Caballero

Maria-Alexandra Paun

Mariachiara Ricci

Mariacrocetta Sambito

Mariah Hoskins

María-José Terrón-López

María-Luisa Pérez-Delgado

Marian Apostol

Marian Brestic

Marian Drusa

Marian Gaiceanu

Marian Ion

Marián Kučera

Marian Lopatka

Marian Marčiš

Marian Šofranko

Marian Vladescu

Marian Wiwart

Marian Wysocki

Mariana Alfaro-Gómez

Mariana Busila

Mariana De Jesus Paiva Proença

Mariana Fraga

Mariana Kolberg

Mariana Marin

Mariana Petrova

Mariangela Vallone

Marianne Maktabi

Marianne Perrin

Marianne Silva

Mariano Bresciani

Mariano Gallo

Mariano Serrao

Mariapia Faruolo

Marica Franzini

Marie Balková

Marie-Josépha Youssef

Mariela Padilla

Marie-Luce Bourguet

Marietta Fodor

Mariia Nazarkevych

Mariia Pukalchik

Marija Brkic Bakaric

Marija Jozanović

Marija Klopčič

Marija Krstic

Marijo Buzuk

Mariko Nakano

Marilena Ronzan
Marin Marin

Marin Marinov

Marina Bolsunovskaya

Marina Bonomolo

Marina Indri

Marina Khazova

Marina Manea

Marina Matos

Marina Miranda

Marina Resta

Marina Rumyantseva

Marina Topa

Marinella Coco

Marinella Giunta

Marinko Barukčić

Marino Vilović

Mario A. Quiroz-Juárez

Mario A.R. Dantas

Mario Alvarado-Lorenzo

Mario Antunes

Mario Cesarelli

Mario Eduardo Rivero-Angeles

Mario Fargnoli

Mario Guevara

Mario Hirz

Mario Iodice

Mário João S. F. Santos

Mário José Gonçalves Cavaco Mendes

Mario Kusek

Mario L. Ruz

Mario Luis Ruz Ruiz

Mario Manzo

Mario Marchetti

Mario Marinelli

Mario Martinez-Zarzuela

Mario Massimo Foglia

Mario Merone

Mario Milicevic

Mario Miscuglio

Mario Montagud

Mário Nuno Mata

Mario Ortiz García

Mario Pardo

Mário Pereira Véstias

Mario Prsa

Mário Reis

Mario Ricardo Gongora-Rubio

Mario Rothbauer

Mário S. Rodrigues

Mario Siciliani

Mario Tamagnone 
Mario Valderrama

Mário Vaz

Mario Verdicchio

Mario Versaci

Mário Véstias

Mário W. L. Moreira

Mario Wallner

Marioara Avram

Mariofanna Milanova

Mariolino De Cecco

Marion Mundt

Marios Anagnostopoulos

Marios Raspopoulos

Marios Sophocleous

Marios Tzouvaras

Marisa Maximiano

Marisa Nicolai

Marius Bumbac

Marius Corici

Marius Gavrilescu

Marius Iulian Mihailescu

Marius Minea

Marius Prelipceanu

Marius Schneider

Marius Vochin

Marius Volmer

Marius-Constantin Vochin

Mariusz Bajger

Mariusz Buciakowski

Mariusz Dąbrowski

Mariusz Druźbicki

Mariusz Giergiel

Mariusz Kłonica

Mariusz Kormanek

Mariusz Kostrzewski

Mariusz Majdański

Mariusz Oszust

Mariusz P. Wilk

Mariusz Pelc

Mariusz Rząsa

Mariusz Specht

Mariusz Szwoch

Mariusz Tryznowski

Mariusz Uchroński

Mariusz Węglarski

Mariusz Zieja

Mariya Konsulova-Bakalova

Marjan Golob

Marjan Mansourvar

Marjan Mernik

Mark A. Arnold
Mark Auslender

Mark Berhow

Mark Carroll

Mark Cronin-Golomb

Mark F. Hansen

Mark J. Potosnak

Mark Jackson

Mark Kenneth Quinn

Mark Levin

Mark Lowry

Mark McCormick

Mark Melnykowycz

Mark Mueller

Mark Potter

Mark Roll

Mark Schulz

Mark T. Marshall

Markéta Potůčková

Markku Löytönen

Marko Bizjak

Marko Hölbl

Marko Jerman

Marko Jurcevic

Marko Katic

Marko Kos

Marko Mäkynen

Marko Malajner

Marko Matulin

Marko Petkovsek

Marko Simic

Marko Stupin

Marko Svaco

Markos G. Tsipouras

Markos Petousis

Markus Buchmann

Markus Feldt

Markus Fiedler

Markus Jobst

Markus M. Hoffmann

Markus Ulmschneider

Markus Vincze

Maros Smondrk

Marouan Mizmizi

Marshed Kassim Mohamed

Mart Min

Marta Bełka

Marta Campos Ferreira

Marta Doval Miñarro

Marta Elena Losa Iglesias

Marta Ferreiro-González

Marta Fiedot-Toboła 
Marta Jarczewska

Marta Kadela

Marta Lorenzini

Marta Peña

Marta Ruiz Llata

Marta Rusnak

Marta Sigut

Marta Sylvia Del Rio

Marta Tessarolo

Marta Wlodarczyk-Sielicka

Marta Zanoletti

Marta Zurek-Mortka

Martha Arbayani Zaidan

Martha Vardaki

Martin Augustynek

Martin Barczyk

Martín Barrère Cambrún

Martin Bauer

Martin Beer

Martin Bitter

Martin Boldt

Martin Boroš

Martin Brandl

Martin Burgdorf

Martin Ćalasan

Martin Č́́žek

Martin Daumiller

Martin Geyer

Martin Goubej

Martin Gulan

Martin Hagmüller

Martin Hempel

Martin Hering-Bertram

Martin J. Strauss

Martin J.-D. Otis

Martin Judd

Martin Kasparick

Martin Kenyeres

Martin Klempa

Martin Kopani

Martin Maguire

Martin Mosko

Martin Otis

Martin Pech

Martin Pollák

Martin Richter

Martin Robinson

Martín Rodríguez

Martin Romero-Sanchez

Martin Rožánek

Martin Schätz
Martin Schmitt

Martin Šipoš

Martin Valtierra-Rodriguez

Martin Velas

Martina Banchelli

Martina Caramenti

Martina Mancini

Martina Medvidović-Kosanović

Martina Teresa Bevacqua

Martina Vettoretti

Martine Neckebroek

Martino Aldrigo

Martino Giaquinto

Martino Marini

Martins Ferreira

Martiwi Diah Setiawati

Martynas Patašius

Marvin Coto-Jiménez

Marvin R. Paulsen

Marx Leandro Naves Silva

Mary Beth Arensberg

Maryam Amini

Maryam Doborjeh

Maryam Salehi

Maryamsadat Tahavori

Marzieh H. Derkani

Masahiko Aki

Masahiro Fujita

Masahiro Seike

Masaki Michihata

Masanori Hara

Masaomi Ikeda

Masashi Sugano

Masato Sone

Masato Yasumoto

Masaya Toda

Masayasu Mie

Masayuki Tanabe

Mashaalah Zarejousheghani

Masoud Barati

Masoud Mirtaheri

Masoud Nazarian-Samani

Massimiliano Benetti

Massimiliano Bordoni

Massimiliano Comisso

Massimiliano Donati

Massimiliano Garda

Massimiliano Pastena

Massimiliano Pepe

Massimo Capoccia

Massimo Conforti 
Massimo Donelli

Massimo Fabris

Massimo Ficco

Massimo Marcaccio

Massimo Montisci

Massimo Musacchio

Massimo Olivero

Massimo Petrarca

Massimo Pieri

Massimo Salvi

Massimo Viviani

Massimo Walter Rivolta

Massimo Zucco

Masudul Haider Imtiaz

Masudul Imtiaz

Mat Dalgleish

Matea Zajc

Matej Mencinger

Matej Njegovec

Matej Supej

Mateja Klun

Mateo Burgos

Mateus Mendes

Mateusz Andrychowicz

Mateusz Bawaj

Mateusz Czyzycki

Mateusz Dybkowski

Mateusz Korpyś

Mateusz Kukla

Mateusz Malanowski

Mateusz Nowak

Mateusz Paliga

Mateusz Pasternak

Mateusz Rzymoeski

Mateusz Salach

Mateusz Zawadzki

Matheus Silva Gonçalves

Mathew G. Pelletier

Mathew L. Wymore

Mathew Legg

Mathias Blandeau

Mathias Forjan

Mathias Lemmens

Mathieu Domenjoud

Mathieu Etienne

Mathieu Gravey

Matias Garcia-Constantino

Matija Jezeršek

Matija Krznar

Matija Milanic

Matjaz Sraml
Matko Šarić

Matloob Khushi

Matrella Guido

Matteo Anedda

Matteo Bolcato

Matteo Bordoni

Matteo Bottin

Matteo Bruno Lodi

Matteo Claudio Palpacelli

Matteo Cortesi

Matteo Cristani

Matteo Del Soldato

Matteo Luperto

Matteo Manganelli

Matteo Martucci

Matteo Moretti

Matteo Riccò

Matteo Saveriano

Matteo Scaramuzza

Matteo Sensi

Matteo Signorile

Matteo Strozzi

Matteo Valt

Matthew A. Cooper

Matthew B. Coppock

Matthew B. Myers

Matthew Brandsema

Matthew Conley

Matthew Eady

Matthew Gadd

Matthew Hobbs

Matthew J. Stainer

Matthew Pain

Matthew Pike

Matthew Scott

Matthew Veale

Matthew Worsey

Matthias Dümpelmann

Matthias Rädle

Matthieu N. Boone

Matti Huotari

Mattia Borgarino

Mattia Brambilla

Mattia Zorzi

Mattias Dahl

Mattias O'Nils

Matúš Pleva

Maura Pavesi

Maurice G. Cox

Maurice Mohr

Maurice Van Keulen 
Mauricio Cerda

Mauricio David Perez

Mauricio J. Silva

Maurício Pamplona Segundo

Mauricio Porath

Maurizio Campolo

Maurizio Magarini

Maurizio Naldi

Maurizio Spadavecchia

Mauro Bellone

Mauro Caccavale

Mauro Callejas Cuervo

Mauro De Feudis

Mauro Epifani

Mauro Fadda

Mauro Fazion Filho

Mauro Feliziani

Mauro Femminella

Mauro Gaspari

Mauro Leonardi

Mauro Lo Brutto

Mauro Mariotti

Mauro Pagano

Mauro Sebastián Innocente

Mauro Sodini

Mauro Tropea

Mavroulis Spyridon

Max M. North

Max Mauro Dias Santos

Max Ortiz-Catalan

Max Ryadnov

Maxim Bakaev

Maxim Dulebenets

Maxim E. Darvin

Maxim Ivanov

Maxim Kalinin

Maxim Khomenko

Maxim V. Ryzhii

Maxime Alex Junior Kuitche

Maxime Bourgain

Maxime Moreaud

Maxime Mouyen

Maximilian Nicolae

Maximo Cobos

Maximo Morales-Cespedes

May Portuguez Castro

Maya Dimitrova

Mayukh Bhattacharyya

Mayuresh Sunil Pardeshi

Maziar Nekovee

Md Alamgir Hossain
Md Asikuzzaman

Md Belal Bin Heyat

Md Eshrat E Alahi

Md Juber Rahman

Md Junayed Hasan

Md Mahbubur Rahman

Md Mominul Ahsan

Md Mostafa Kamal Sarker

Md Noor-A-Rahim

Md Shamsuddoha

Md Sipon Miah

Md Tanvir Hasan

Md Zafar Iqbal

Md. Al-Amin Khandaker

Md. Faruk Hossain

Md. Humayun Kabir

Md. Sanaul Haque Mondal

Med Aymen Challouf

Mehanathan Pathmanathan

Mehdi A J Van Den Bos

Mehdi Adda

Mehdi Boukallel

Mehdi Boukhechba

Mehdi Eshagh

Mehdi Foumani

Mehdi Ghoreyshi

Mehdi Hosseinzadeh

Mehdi Nosrati

Mehdi Pazhoohesh

Mehdi Pirahandeh

Mehdi Rahmati

Mehdi Roozmeh

Mehdi Salimi

Mehedi Masud

Mehmet Alp Ilgaz

Mehmet Karakose

Mehmet Ogut

Mehmet Senel

Mehran Behjati

Mehran Mehrandezh

Mehran Oraee

Mehrmohammadi Mohammad

Mehtab Singh

Mei Pan

Mei Sang

Meicheng Li

Meikang Han

Meimei Lai

Meir Nitzan

Meiwei Kong

Meizhen Wang 
Mel Suffet

Melania Susi

Melania Tera

Melanie Elias

Melanie Haehnel-Taguchi

Melina P. Ioannidou

Melina-Maria Zempila

Melinda David

Melisa Del Barrio

Melissa M. Rodríguez-Delgado

Melkie Tadesse

Meltem Elitas

Meltem Yanilmaz

Melvin Diaz

Meng Gao

Meng Han

Meng Li

Meng Lin

Meng Zhou

Mengbao Fan

Mengchu Zhou

Mengfang Li

Meng-Fang Lin

Menghan $\mathrm{Hu}$

Mengistie Kindu

Mengjie Han

Mengjie Shi

Meng-Kun (Jason) Liu

Menglan Duan

Meng-Leong How

Menglong Liu

Mengmi Zhang

Mengyan Nie

Meng-Yun Chung

Meonghun Lee

Mercedes Ayuso

Mercedes Del Río Celestino

Mercedes Ruiz

Mercedes Solla

Merryn Cole

Mert Sevil

Merve Keskin

Meysam Keshavarz

Mezgeen Rasol

Mhafuzul Islam

Mhamed Sayyouri

Mi Chen

Mia Folke

Miao Hu

Miao Lu

Miao Yu
Miaoqiang Lyu

Miaowen Wen

Micael Nascimento

Micaela Morettini

Micaela Schmid

Michael A. Cowling

Michael A. Lombardi

Michael Arand

Michael Baddeley

Michael Balchanos

Michael Baldauf

Michael Barz

Michael Borich

Michael Coughlin

Michael D. Gray

Michael DeGiorgio

Michael Döllinger

Michael Domingue

Michael Ellison

Michael Farmer

Michael Fowler

Michael Friebe

Michael G. Mauk

Michael Garstang

Michael Gould

Michael H. F. Wilkinson

Michael Hahn

Michael Haupt

Michael Heise

Michael Hewson

Michael Höft

Michael I. Ojovan

Michael J. Abrams

Michael J. Wouters

Michael James Price

Michael Kirchhoff

Michael Kuntzsch

Michael L. Myrick

Michael Lata

Michael MacDonald

Michael Mangold

Michael Marx

Michael McGuire

Michael Meyer

Michael Mielewczik

Michael Mortimer

Michael Mück

Michael Muehlberghuber

Michael Munz

Michael Nikolaou

Michael O'Byrne 
Michael O'Toole

Michael Osadebey

Michael P. Caligiuri

Michael P. Craven

Michael R. Koblischka

Michael Reader-Harris

Michael Ristic

Michael Sakellariou

Michael Semenov

Michael Shribak

Michael Thompson

Michael Trevino

Michael Vannier

Michael Werman

Michael Winokur

Michael Yu Kataev

Michaela Balažiková

Michaela Servi

Michail Gianniou

Michail J. Beliatis

Michail Klontzas

Michail-Alexandros Kourtis

Michal Balazia

Michal Borecki

Michał Brach

Michal Dolezel

Michal Dudek

Michał Fularz

Michal Gála

Michał Ginszt

Michal Holub

Michal Huptych

Michał Józwik

Michał Jurek

Michal Kaczmarek

Michal Kebisek

Michal Kelemen

Michał Kunicki

Michal Kvet

Michał Landowski

Michał Morawski

Michał Nowicki

Michał Pająk

Michal Piasecki

Michal Podpora

Michał R. Nowicki

Michał R. Wróbel

Michał Rogala

Michał Romaszewski

Michal Schmirler

Michał Śmieja
Michal Strzelecki

Michal Szermer

Michal Wieczorowski

Michał Wojasiński

Michal Wydra

Michalina Błażkiewicz

Michalis A. Savelonas

Michalis Kassinopoulos

Michalis P. Michaelides

Michalis Savelonas

Michalis Vrigkas

Michalski Andrzej

Michel Eustáquio Dantas Chaves

Michel Gay

Michel Jourlin

Michel Kasser

Michel Misson

Michel Nielen

Michel Paindavoine

Michel Riguidel

Michel Salomon

Michela Borghetti

Michela Goffredo

Michela Quadrini

Michelangelo Guaitolini

Michele Arturo Caponero

Michele Boffano

Michele Calì

Michele Carboni

Michele Colajanni

Michele D'Attilio

Michele De Santis

Michele Dei

Michele Girolami

Michèle Gouiffès

Michele Mangiameli

Michele Mastroianni

Michele Ortolani

Michele Pisante

Michele Roccotelli

Michele Scandola

Michelle Liou

Michelle Mellenthin

Michio Niwano

Migallón Héctor

Miguel A. Jaramillo-Morán

Miguel A. Santoyo

Miguel Ángel Caminero Torija

Miguel Ángel Fernández Torres

Miguel Angel Fernandez-Granero

Miguel Ángel Gómez Ruano 
Miguél Ángel Gutierrez

Miguel Ángel López

Miguel Angel Luque-Nieto

Miguel Ángel Maté González

Miguel Ángel Naya Villaverde

Miguel Ángel Satorre

Miguel Carrasco

Miguel Castelo-Branc Sousa

Miguel Chavarrías

Miguel Clavijo Jiménez

Miguel Díaz-Rodríguez

Miguel Dovale Alvarez

Miguel Franklin De Castro

Miguel Gabriel Villarreal-Cervantes

Miguel García-Pineda

Miguel León-Rodríguez

Miguel Martínez García

Miguel Maté-González

Miguel Murillo-Escobar

Miguel Navarrete

Miguel Ossandon

Miguel Pic Aguilar

Miguel R. Oliveira Panão

Miguel Reis E. Silva

Miguel Rodríguez Pérez

Miguel Torre García

Miguel Torres

Miguel-Angel Luque-Nieto

Miha Ambrož

Miha Ravber

Mihael Makek

Mihael Mohorcic

Mihaela Andrei

Mihaela Bacalum

Mihaela Badea Doni

Mihaela Balanescu

Mihaela Colhon

Mihaela Cretu

Mihaela Gheorghiu

Mihaela Hnatiu

Mihaela Ioana Baritz

Mihaela Oleksik

Mihaela Puiu

Mihaela Tertiş

Mihaela-Ligia Unguresan

Mihai Ciprian Mărgărint

Mihai Ciuc

Mihai Crenganis

Mihai Gavrilas

Mihai Olimpiu Tatar

Mihai Sanduleanu
Mihai Udrescu

Mihai Victor Pricop

Mihai Zaharia

Mihaiela Iliescu

Mihail Abrudean

Mihail Iancovici

Mihail Minescu

Mihalj Bakator

Mikael Forsman

Mikael Luthje

Mikail F. Lumentut

Mikalai M. Budzevich

Mike Adams

Mike Myers

Mike Oluwatayo Ojo

Mikel Emaldi

Mikel Ogueta-Gutiérrez

Mikhail Basarab

Mikhail Basov

Mikhail Beklemishev

Mikhail Belovolov

Mikhail Bogachev

Mikhail Evgen'evich Prokhorov

Mikhail Kirillin

Mikhail Komarov

Mikhail Krinitskiy

Mikhail Lytaev

Mikhail P. Kozochkin

Mikhail Skvortsov

Mikhail Slobodyan

Mikhail Tarkov

Mikhail Zolotukhin

Mikio Deguchi

Mikko Peltokangas

Miklos Fried

Mikolaj Buchwald

Mikołaj Karpiński

Mikuláš Huba

Milad Gholami

Milad Heydariaan

Milad Kamkar

Milad Karimshoushtari

Milan Červenka

Milan Durdán

Milan Džunda

Milan Gnjatović

Milan Polivka

Milan Smetana

Milan Sys

Milan Toma

Miled Amine 
Milena B. Čukić

Milena Faria Pinto

Milena Marković

Miles Grafton

Milica Jankovic

Miloš Milovanović

Miltiadis Kandias

Milto Miltiadou

Milton Hirokazu Shimabukuro

Milton Pereira

Miltos Kyriakidis

Milutin Radonjic

Mimi Recker

Min Kim

Min Li

Min Pan

Min Park

Min Seok Moon

Min Tu

Min Xia

Min Xie

Min Xu

Mina Samukawa

Mina Sheikhalishahi

Minas Dasygenis

Minas Patsalides

Minchae Jung

Mindy Levine

Mindy S. Levine

Minfang Yeh

Ming Liu

Ming Tzer Lin

Ming Yan

Ming Yang

Ming $\mathrm{Yu}$

Ming Yuan

Ming-Bao Huang

Ming-Chang Pai

Mingchao Li

Mingchen Zhuge

Ming-Chin Chuang

Mingda Zhou

Mingde Du

Mingder Yang

Ming-Feng Ge

Ming-Fong Tsai

Minghsun $\mathrm{Wu}$

Minghui Li

Ming-Hung Hsu

Ming-Hung Wang

Ming-Jer Jeng
Ming-Jie Sun

Mingjie Yin

Mingjie Zhang

Mingkui Wu

Mingliang Zhou

Mingmin Zhao

Mingsen Pan

Mingsheng Ma

Mingshun Jiang

Ming-Shyan Wang

Mingsong Wang

Mingsu Si

Ming-wei Hsu

Mingxuan Chen

Mingyang Lu

Ming-Yang Su

Mingye Ju

Ming-Yen Hsiao

Mingyu Lim

Mingyuan Yan

Mingzhang Chen

Mingzhe Jiang

Mingzhe Zhang

Min-Ho Lee

Minhong Sun

Minh-Quang Tran

Minh-Tu Cao (Luis)

Minjeong $\mathrm{Ha}$

Minjie Guo

Minjoong Rim

Min-Jung Kang

Minkoo Kim

Min-Kook Choi

Minkyu Ahn

Minkyu Song

Minna Lanz

Mino Sportelli

Minoru Sasaki

Minseok Choi

Min-Seok Kang

Minshui Huang

Minvydas Ragulskis

Minxiang Zeng

Miquel Sànchez-Marrè

Mira Naftaly

Miralda Cuka

Miran Lee

Miran Merhar

Miran Pobar

Mircea Cristian Dudescu

Mircea Dorin Vasilescu 
Mircea Dulău

Mircea Hulea

Mircea Ivanescu

Mircea Neagoe

Mircea Paul Muresan

Mircea Vladutiu

Mircea-Bogdan Radac

Mirco Muttillo

Mirco Sturari

Mirela Catalina

Mirela Frandes

Mirella De Sisto

MirHojjat Seyedi

Miriam A. Carlos-Mancilla

Miriam Zachsenhouse

Mirja Tenhunen

Mirjana Pejić Bach

Mirko De Melis

Mirko Marras

Mirko Mazzoleni

Mirko Mustonen

Mirko Poljak

Mirko Viroli

Mirna Habuda-Stanić

Mirna Žulec

Miron Weinreb

Miroslav Bures

Miroslav Císar

Miroslav Jůzl

Miroslav Kulich

Miroslav Pástor

Miroslav Pohanka

Miroslav Pokorný

Miroslav Voznak

Miroslav Živić

Miroslava Nedyalkova

Miroslaw Mazurek

Mirosław Nejman

Mirosław Pajor

Miroslaw Swiercz

Mirosław Szmajda

Miroslaw Witos

Mirza Pojskić

Mirza Sarajlic

Misa Hayashida

Mislav Stepinac

Mitch Pryor

Mitchell L. Neilsen

Mitchell Welch

Mithun Kuniyil Ajith Singh

Mithun Mukherjee
Mitja Truntič

Mitra Subhabrata

Mitrea Delia-Alexandrina

Mitsugu Hasegawa

Mitsuhiro Shigeishi

Mittal Mohit

Mizanoor Rahman

Mizuho Nishio

Mladen Amović

Mladen Jurišić

Mladen Zrinjski

Młynarczyk Przemysław

MM Manjurul Islam

Mo Zhao

Moacir Moacir Godoy

Moayad Aloqaily

Mobayode Akinsolu

Mocanu Irina

Mochimitsu Komori

Modestos Stavrakis

Modris Greitans

Mogalahalli V. Reddy

Mohamad Alzayed

Mohamad Awad

Mohamad Sawan

Mohamadali Malakoutian

Mohamed Abd Elaziz

Mohamed Abdel-Nasser

Mohamed Abdelrahem

Mohamed Amine Ferrag

Mohamed Atef Abbas Elsaharty

Mohamed Ben Haj Frej

Mohamed Benbouzid

Mohamed Bouazaoui

Mohamed Boutaayamou

Mohamed Dahmane

Mohamed Darwish

Mohamed E. Tamazin

Mohamed Elhabiby

Mohamed El-Hadidy

Mohamed Fnadi

Mohamed Foysol Chowdhury

Mohamed Hamdaoui

Mohamed Hammad

Mohamed Saad

Mohamed Salahudeen

Mohamed Serry

Mohamed Sharafeldin

Mohamed Shehadeh

Mohamed Shehata

Mohamed Tamazin 
Mohamed Wiem Mkaouer

Mohamed Zaghloul

Mohammad A. Aljamal

Mohammad A. Jaradat

Mohammad A. Maktoomi

Mohammad Alibakhshikenari

Mohammad Bagher Dowlatshahi

Mohammad Belal

Mohammad Faridul Haque Siddiqui

Mohammad H. Alomari

Mohammad H. Nadimi-Shahraki

Mohammad Hammoudeh

Mohammad Harb

Mohammad Hossein Amirhosseini

Mohammad Iman Mokhlespour Esfahani

Mohammad Jafari

Mohammad Jamshidi

Mohammad Jobayer Hossain

Mohammad Kakooei

Mohammad Kamrul Hasan

Mohammad Khishe

Mohammad Khubeb Siddiqui

Mohammad Mahdi Rezapour Mashhadi

Mohammad Mahdi Tajiki

Mohammad Mehdi Rashidi

Mohammad Mehedi Hasan

Mohammad Miralinaghi

Mohammad Moghimi

Mohammad Nadimi

Mohammad Najafzadeh

Mohammad Noori

Mohammad Reza Jabbarpour

Mohammad Reza Karafi

Mohammad Reza Khosravani

Mohammad Reza Mosavi

Mohammad Reza Salami

Mohammad Rmayti

Mohammad Rostami

Mohammad Saeid Ghaffarian

Mohammad Safeea

Mohammad Shojafar

Mohammad Shokrolah Shirazi

Mohammad Soleymani

Mohammad Taghinejad

Mohammad Tavakkoli Yaraki

Mohammad Valipour

Mohammad Wazid

Mohammad Yazdani Asrami

Mohammadali Ghafarian

Mohammadreza Shokouhimehr

Mohammadreza Soltaninejad
Mohammed A. A. Al-qaness

Mohammed Abuhamad

Mohammed Al Sadsoon

Mohammed Alhaddad

Mohammed Amin Almaiah

Mohammed Awad

Mohammed Bahja

Mohammed Baz

Mohammed Chadli

Mohammed Diykh

Mohammed Elmogy

Mohammed H. Alsharif

Mohammed Ijaz

Mohammed Mahmoud

Mohammed Salah Al-Radhi

Mohammed Salih Mohammed Gismalla

Mohand Arab Djeziri

Mohataz Hossain

Mohd Anuaruddin Bin Ahmadon

Mohd Azizi Abdul Rahman

Mohd Noor Islam

Mohd Saiful Azimi Bin Mahmud

Mohd Shahrimie Mohd Asaari

Mohit Mittal

Mohit Saraf

Mohiuddin Ahmed

Mohsen Asadnia

Mohsen Azarmi

Mohsen Kaboli

Mohsen Koohestani

Mohsen Ranjbaran

Mohsen Rezvani

Mohsin Jamil

Moi Hoon Yap

Moin U. Atique

Moinak Maiti

Mojca Urbancic

Mojtaba Ahmadieh Khanesar

Mojtaba Forghani

Mojtaba Ghodsi

Mojtaba Jafari Tadi

Mojtaba Joodaki

Mojtaba Khanesar

Mojtaba Noghabaei

Mojtaba Zeraatpisheh

Molnár Bence

Molong Duan

Mona Ionas

Mona Jaber

Monica Ballesta Galdeano

Monica Betta 
Mónica Fernández Barciela

Monica Figueiredo

Monica Florescu

Monica Löfvander

Monica Marini

Monica Necchi

Monica Pătrașcu

Mónica Pinto

Monica Siroux

Monica Tiboni

Monika Błaszczyszyn

Monika Cieslikiewicz-Bouet

Monika Gelker

Monika Gwóźdź-Lasoń

Monika Sobiech

Monssef Drissi-Habti

Monsur Islam

Montañes Rosa Rodriguez

Montserrat Calleja

Moon Il Kim

Moon K. Kwak

Moon-Kyu Lee

Moonseong Kim

Moonsoo Kang

Moreno D'Amico

Morgan Funderburk

Moritz Hess

Moritz S. Schmid

Morshed Chowdhury

Morteza Babaie

Morteza Ghorbani

Moshe Gai

Moshe Rav Acha

Mostafa Elgendy

Mostafa Ghobaei-Arani

Mostafa Haghi

Mostafa Hassanalian

Mostafa Hussein

Mostafa Kamal Masud

Mostafa Nabawy

Mostafa Sadeghi

Mostefa Mohamed-Seghir

Motoaki Hara

Motoya Suzuki

Mouad Lemoudden

Moussa Hamadache

Moustafa Hussein

Mrinal Mandal

Mu-chun Wang

Mueen Uddin

Mufti Mahmud
Mugahed A. Al-antari

Muguang Wang

Muhammad Aamir Cheema

Muhammad Abdul Qyyum

Muhammad Afaq

Muhammad Afzal

Muhammad Ahmad

Muhammad Ahsan Saeed

Muhammad Ali Butt

Muhammad Amir Khan

Muhammad Amir Shafiq

Muhammad Amith

Muhammad Anshari

Muhammad Arif Mahmood

Muhammad Asad

Muhammad Asghar Khan

Muhammad Ashfaq Jiskani

Muhammad Ashfaq Khan

Muhammad Asif Zahoor Raja

Muhammad Aslam

Muhammad Attique

Muhammad Attique Khan

Muhammad Awais

Muhammad Aziz

Muhammad Azmat

Muhammad Ehatisham-ul-Haq

Muhammad Fahad Zia

Muhammad Faheem

Muhammad Fazal Ijaz

Muhammad Hassan Khan

Muhammad Hussain

Muhammad Ikram

Muhammad Kashif

Muhammad Kashif Shahid

Muhammad Khalil Afzal

Muhammad Mahmood Ali

Muhammad Nadeem

Muhammad Naveed Aman

Muhammad Naveed Aman Aman

Muhammad Nazir

Muhammad Raheel Bhutta

Muhammad Rashid

Muhammad Sadiq Fareed

Muhammad Saif Ullah Khalid

Muhammad Salman Al Farisi

Muhammad Salman Haleem

Muhammad Shafiq

Muhammad Shuja Khan

Muhammad Usman Hadi

Muhammad Usman Younus

Muhammad Yousuf Irfan Zia 
Muhammad Zubair

Muhammd Mudassar Yamin

Muhammed Said Boybay

Muharfiza Muharfiza

Muharrem Kemal Özfırat

Muhib Rahman

Muhidul Islam Khan

Mujtahid Kaavessina

Mukesh Kumar

Mukesh Kumar Sriwastva

Mukesh Kumar Thakur

Mukesh Prasad

Mukhtar Abdulkadir

Mulugeta Wayu

Mumin Cebe

Mumtaz Hussain Soomro

Mun'delanji C. Vestergaard

Muneeb Ahmad

Muneesh Maheshwari

Munish Kumar Gupta

Murad Khan

Murat Kaya Yapici

Murat Simsek

Murillo Ferreira Dos Santos

Murilo Araujo Romero

Murilo Bellezoni Loiola

Murtadha Hssayeni

Murthy Chavali

Musab Hameed

Mustafa Rajabali

Mustapha Jouiad

Mustapha Maâtouk

Mustaqeem Mustaqeem

Mutaz Barika

Muthaiah Shellaiah

Muthoni Masinde

Muthu Ram Prabhu Elenchezhian

Muthuchamy Nallal

Muzaffar Rao

Myeong-Hun Jeong

Myeounggon Lee

Mykola Sysyn

Myounggon Kang

Myounggyu Won

Myriam Zerrad

Myung Hwan Park

Myungjun Kim

Myung-Ki Kim

Myung-Sup Kim

Nabamita Pal

Nabeel Mohammed
Nabil Ashraf

Nabil Bachagha

Nabila Brihmat

Nachappa Gopalsami

Nachiket Tapas

Nadarajah Manivannan

Nader Naghavi

Nader Shehata

Nader Taheri-Qazvini

Nadezhda Yarushkina

Nadia Belu

Nadia Delavarpour

Nadia Kanwal

Nadica Miljković

Nadir Kapetanović

Nadir Shah

Nadjim Horri

Nadra Guizani

Naeem Ayoub

Naeem Iqbal

Nafati Aboserwal

Nafees Mansoor

Nag Anindya

Nagaraj Yamanakkanavar

Nageswara Lalam

Nagham Saeed

Naghmeh Mahmoodian

Nagy Lajos

Nahid Majd

Nahina Islam

Naiara Demnitz

Naim Mitre

Najib Kacem

Najmeh Karimian

Nakju Doh

Nakul Pande

Nalika Ulapane

Nallapaneni Manoj Kumar

Nallappan Gunasekaran

Nam Huynh

Nam Kim

Nam Tuan Le

Namhoon Kim

Namhyuk Ham

Nam-Yong Lee

Nan Jiang

Nan Li

Nan Qi

$\mathrm{Nan} \mathrm{Wu}$

Nan Xu

Nan Yang 


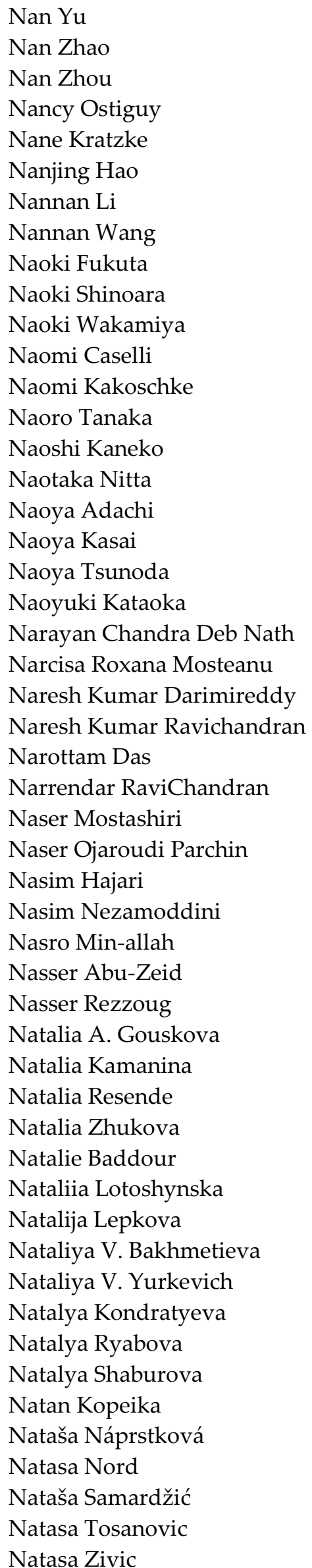

Natassya Silva

Nathalie Sauer

Nathan Lau

Nathan Lazarus

Nathan Salowitz

Nathaniel Hunt

Nathir A. Rawashdeh

Nauman Khalid Qureshi

Navid Mojtabai

Navid Reyhanian

Nawab Muhammad Faseeh Qureshi

Nawaf Hamadneh

Nawal Elfishawy

Nawar Kadi

Nayden Nenkov

Nazakat Ali

Nazanin Neshatvar

Nazarena Bruno

Nazek El-Atab

Nazia Hameed

Nazmul Beg

Nebahat Şule Üstün

Nebojsa Doncov

Nebojša Malešević

Neda Haj-Hosseini

Nediljka Gaurina-Međimurec

Nédra Mellouli

Neeraj Bokde

Nefta-Eleftheria Votsi

Neil Boonham

Neil Collings

Neil Glossop

Neil Grigg

Neil I. Fox

Nekane Sainz

Nel Samama

Nelcio Antonio Tonizza De Carvalho

Nélia Alberto

Nelly Leligou

Nelson Batista

Nemai Chandra Karmakar

Nenad Gligoric

Nengchao Lyu

Nengfang Chao

Nerija Zurauskiene

Nerijus Morkevicius

Nestor Arana Arexolaleiba

Nestor Cabrera-Munoz

Néstor José Jarque Bou

Nestor Montalvan-Burbano

Neven Vrček 
Newcomb Robert

Ngoc-Huynh Ho

Nguyen Dinh Dung

Nguyen Ngoc-Phi

Nguyen Quang Vinh

Nguyen Quoc Khanh Le

Nguyen Thanh-Vinh

Nguyen Thi Phuoc Van

Nguyen Truong

Nhat-Duc Hoang

Niall Higgins

Niall O'Mahony

Niamat Hussain

Nianyin Zeng

Nibaldo Rodriguez

Niccolo Baldanzini

Niccolò Dematteis

Nicholas A. Giudice

Nicholas Caporusso

Nicholas Chileshe

Nicholas J. Kinar

Nicholas J. Long

Nicholas Jonsson

Nicholas Oesch

Nicholas Rotella

Nicholas Samaras

Nicholas Vincent Apollo

Nicholas Vretos

Nicholas X. Williams

Nick Andrei Ivănescu

Nick Donaldson

Nick Draper

Nick Pitropakis

Nicola A Morley

Nicola Bellomo

Nicola Giaquinto

Nicola Liberatore

Nicola Lopomo

Nicola Ludwig

Nicola Marotta

Nicola Massari

Nicola Orzalesi

Nicola Pellegrini

Nicola Schulz

Nicola Testoni

Nicola Zema

Nicolae Constantinescu

Nicolae Goga

Nicolae Herisanu

Nicolae Pop

Nicolae Tudoroiu
Nicolae-Doru Stănescu

Nicolai Spicher

Nicolas Alexander Tatlas

Nicolas Auclair

Nicolas Dalmedico

Nicolas Garcia-Aracil

Nicolás Laguarda-Miró

Nicolas Le Sommer

Nicolas P. Avdelidis

Nicolas Roehri

Nicolas Spinelli

Nicolas Stouls

Nicolas Verrier

Nicole Jaffrezic-Renault

Nicole W. Xu

Nicole Zahradka

Nicoleta Enescu

Nicoleta Gillich

Nicoleta Ilies

Nicoletta Nicolaou

Nicolo Beverini

Nicolò Maccaferri

Nicolo Marconato

Nicos Maglaveras

Nicu Constantin Tudose

Nihan Aydemir

Nihanth W. Cherukuru

Nijolè Batarlienè

Nikhil Bhalla

Nikita Andriyanov

Nikita Minaev

Nikita Shepelin

Nikitas Nikitakos

Niko Lukač

Nikola Anđelić

Nikola Banic

Nikola Baran

Nikola Gradojevic

Nikola Ivković

Nikola Kranjčić

Nikola Lopac

Nikola Sakač

Nikola Zogović

Nikolai Kornev

Nikolai Perov

Nikolai Stoianov

Nikolai Tsvetkov

Nikolai Ushakov

Nikolaj Goranin

Nikolaos A. Androutsos

Nikolaos Athanasios Anagnostopoulos 
Nikolaos C. Kokkinos

Nikolaos Dimitriou

Nikolaos Doulamis

Nikolaos Giannakeas

Nikolaos K. Lyras

Nikolaos Kantartzis

Nikolaos Karathanasopoulos

Nikolaos Mavridis

Nikolaos Mitianoudis

Nikolaos Nikolakis

Nikolaos Nomikos

Nikolaos Pitropakis

Nikolaos T. Milas

Nikolaos Tapoglou

Nikolaos Tsalis

Nikolaos V. Kantartzis

Nikolaos Vitzilaios

Nikolaos-Alexandros Tatlas

Nikolas Pontikos

Nikolay Borodinov

Nikolay Kakanakov

Nikolay Kazanskiy

Nikolay Mukhin

Nikolay Shilov

Nikolay Smagin

Nikolay Todorov Atanasov

Nikolina Jankovic

Nikos A Chaniotakis

Nikos Antonopoulos

Nikos Dimitriou

Nikos Dimitris Fakotakis

Nikos Konofaos

Nikos Petrellis

Nikos Svigkas

Nikunj A. Bhagat

Nili Krausz

Nils Tångefjord Basse

Nils Von Neuhoff

Nima Javanbakht

Nima Karimian

Nima Movahedi

Nimrod Carmon

Nimsiri Abhayasinghe

Nina Danilina

Nina Rizun

Nina Sinatra

Ninad Mehendale

Ning $\mathrm{Hu}$

Ning Li

Ning Liu

Ning Sun
Ning Wang

Ning $X i$

Ning Zhao

Ningbo Yu

Ning-de Jin

Ningning Han

Ningqi Luo

Nino F. Läubli

Ninoslav Majurec

Nirav Joshi

Niroshinie Fernando

Nirvana Popescu

Nirvedh Meshram

Nishant Tripathi

Nishanth Venugopal Menon

Nishikawa Yuichi

Nita Yodo

Nitish Katoch

Nitish Thakor

Nitu Syed

Nivaldo T. Schiefler

Nivedita Nivedita

Nizam Uddin Ahamed

Nizar Jaber

Noa Garcia

Noé Pérez-Higueras

Noel Bristow

Noelia Zagalaz Anula

Nojan Aliahmad

Nolwenn Lapierre

Noman Naseer

Noor Zaman Jhanjhi

Noora Isoaho

Nor Shahida Mohd Shah

Nóra Baranyai

Noramaliza Mohd Noor

Norbert Chamier-Gliszczyński

Norbert Lindlein

Norbert Palka

Norbert Schwesinger

Norbert Wehn

Nordin Rosdiadee

Norihiro Kamamichi

Noriko Saito

Norio Miyoshi

Norio Tagawa

Noriyuki Kawarazaki

Noriyuki Kimura

Norma-Aurea Rangel-Vázquez

Norman Adams

Noshin Fatima 
Nourdine Aliane

Noushin Nasiri

Nozhan Bayat

Nozomi Nishizawa

Nuggehalli M. Ravindra

Nuno Alexandre Ribeiro Costa

Nuno Cruz

Nuno Gonçalves

Nuno Mendes

Nuno Miguel Fonseca Ferreira

Nuno Morais

Nuno Pessanha Santos

Nuno Rodrigues

Nunzio Torrisi

Nuria Duffo

Núria Gavara

Nuria Novas Castellano

Nuria Teigell Benéitez

Nurul Sarkar

Nusret Demir

Nuvia M. Saucedo

Oana Geman

Oana Hosu

Oana Mitruț

Obada Al Zoubi

Octavia Dobre

Octavian Dospinescu

Octavian Duliu

Octavian Fratu

Octavian Postolache

Octavio Gutierrez Frias

Oday Ibraheem Abdullah

Oded Liran

Odongo Steven Eyobu

Ofer Barnea

Ognjen Arandjelović

Ognjen Orozovic

Oktay Yilmazoglu

Okur Salih

Okuwobi Idowu Paul

Okyay Altay

Olaf Gefeller

Olaf Ueberschär

Oláh Judit

Olamide Timothy Tawose

Olatunji Mumini Omisore

Olatunji.Abdul Shobande

Olav Egeland

Ole Bang

Oleg A. Markelov

Oleg A. Stepanov
Oleg Golovnin

Oleg I Lyamin

Oleg Illiashenko

Oleg K. Golovnin

Oleg M. Gradov

Oleg Morozov

Oleg Myakinin

Oleg Olegovich Evsutin

Oleg Sergiyenko

Oleg Shiryayev

Oleg Stukach

Oleg Udalov

Oleg V. Butov

Oleg V. Ivanov

Oleg V. Zakharov

Oleksandr Makeyev

Oleksandr Malyuskin

Oleksandr Molnar

Oleksiy Sydoruk

Olga Bibikova

Olga Boytsova

Olga Cherkasova

Olga E. Glukhova

Olga Fragou

Olga Korostynska

Olga Kurasova

Olga Sergeeva

Olga Sokolova

Olga Zinovieva

Olga-Joan Ktenidou

Olimpiu Stoicuță

Oliver C. Saavedra

Oliver Cuate

Oliver Faust

Oliver Michler

Oliver Ozioko

Olivér Törő

Olivia Curzio

Olivier Dalverny

Olivier Debeir

Olivier J.N. Bertrand

Olivier Marcelot

Olivier Schalm

Olli Jokinen

Oludayo Olugbara

Olumide Oluyisola

Oluwakayode Onireti

Oluwatosin Oluwadare

Om Jee Pandey

Omar Abdul Wahab

Omar Aguilar 
Omar Durrah

Omar Hamza

Omar Janeh

Omar Longoria-Gandara

Omar Nibouche

Ömer Muhammet Soysal

Omid Akhavan

Omid Zargar

Omneya Attallah

Omprakash Kaiwartya

Ona Lukoševičienè

Ondrej Fišer

Ondrej Hanušovský

Ondrej Krejcar

Ondrej Stopka

Ondřej Straka

Ondřej Vondrouš

Ondřej Životský

Onel Luis Alcaraz López

Onur Alev

Onur Toker

Oonagh M Giggins

Orazio Aiello

Orcan Alpar

Orestis Georgiou

Orhan Ozguner

Oriel Frigo

Ørjan G. Martinsen

Orlando Camargo Rodríguez

Orsolya Kiss

Ortner Michael

Oscar Adamuz-Hinojosa

Oscar Andres Vivas

Óscar Barquero-Pérez

Óscar Belmonte Fernández

Oscar Borla

Óscar Carranza

Oscar Casas

Oscar Castillo

Óscar De Francisco Ortiz

Óscar DelCastillo-Andrés

Oscar Duque

Oscar Ernesto-Casanova

Oscar Gutierrez-Blanco

Oscar Herrera-Alcántara

Oscar Kjell

Oscar Nunez

Oscar Reinoso

Oskar Marko

Oskar Sterle

Oskar Szumski
Osvaldo Gervasi

Oswaldo Baffa

Oswaldo Hideo Ando Junior

Oswaldo Lopez-Santos

Oswaldo Morales Matamoros

Oswaldo Ulises Juarez-Sandoval

Otakar Horejš

Otar Akanyeti

Otilia Bobis

Otman Mrabet

Otniel Portillo Rodríguez

Oto Haffner

Otoniel Mario López Granado

Ottavia Giuffre

Otto Kauffman

Ouk Choi

Ouyang Xiaoying

Oveis Amir Hosein

Ovidio Salvetti

Ovidiu Bordean

Ovidiu Ivanov

Ovidiu Stan

Oxana Verkholyak

Oyunchimeg Shagdar

Ozgur Erkent

Özkan Uğurlu

Ozminkowski Ron

Paavo Nevalainen

Pabasara Wanniarachchige

Pablo A. Garcia-Salaberri

Pablo Angueira

Pablo Aqueveque

Pablo Cano Marchal

Pablo Corral

Pablo Escobedo

Pablo Fernandez Miaja

Pablo Giménez-Gómez

Pablo Horstrand

Pablo Luis López Espí

Pablo Manuel Millán-Millan

Pablo Martinez

Pablo Muniz

Pablo Otero

Pablo Rodriguez Gonzalvez

Pablo Rodríguez Luque

Pablo Venegas

Pagger Ernst Peter

Pai-Hsun Chen

Pal Varga

Palahin Volodymyr

Palani Elumalai 
Palanivel Kandasamy

Pamela Zontone

Panagiota-Kyriaki Revelou

Panagiotis A. Eliopoulos

Panagiotis A. Karkazis

Panagiotis Agrafiotis

Panagiotis Alevras

Panagiotis Barmpoutis

Panagiotis E. Pintelas

Panagiotis Georgakis

Panagiotis Gkonis

Panagiotis K. Gkonis

Panagiotis Karkazis

Panagiotis Karmiris-Obratański

Panagiotis Kourtesis

Panagiotis Kyratsis

Panagiotis Papageorgas

Panagiotis Partsinevelos

Panagiotis Radoglou-Grammatikis

Panagiotis Sarigiannidis

Panagiotis Stavropoulos

Panagiotis Trakadas

Panagiotis Tsarabaris

Pandian Pitchipoo

Pang-Wei Tsai

Pankaj Bhowmik

Pankaj Singh

Panos Achlioptas

Panos Fitsilis

Pantelis Frangoudis

Paola Di Pietro

Paola Ferrario

Paola Saccomandi

Paola Sansoni

Paolo Bellagente

Paolo Bertoncello

Paolo Casari

Paolo Castaldi

Paolo Castaldo

Paolo Castellini

Paolo Cavoretto

Paolo Crippa

Paolo Di Lillo

Paolo Facci

Paolo Finocchiaro

Paolo Formichi

Paolo Fraccaro

Paolo Gastaldo

Paolo Giammatteo

Paolo Intini

Paolo Jose Cesare Biselli
Paolo Lino

Paolo Melillo

Paolo Mercorelli

Paolo Meriggi

Paolo Motto Ros

Paolo Nepa

Paolo Pascolo

Paolo Pasquali

Paolo Petrini

Paolo Sernani

Paolo Sommella

Paolo Spagnolo

Paolo Trucillo

Paolo Visconti

Paolo Zaffino

Paolo Zuccon

Parameshachari B D

Parashar Dhakal

Parastoo Farnia

Pari Delir Haghighi

Paria Esmatloo

Parikshit Dutta

Parinaz Abiri

Parsa Omidi

Partarakis Nikolaos

Parth Bhavsar

Parthiban Pazhamalai

Parthiban Rajan

Parviz Safadel

Pascal Acco

Pascal Aubry

Pascal Dupuis

Pascal Hirmer

Pascal Nicolay

Pascal Peter

Pascal Schirmer

Pasquale Cianci

Pasquale D'Angelo

Pasquale De Meo

Pasquale Di Palma

Pasquale Giungato

Pasquale Tommasino

Patrice Forget

Patrice Megret

Patrice Salzenstein

Patrícia A. Pereira

Patricia Arroyo

Patricia Broderick

Patricia Dolez

Patricia Jovičević-Klug

Patricia Khashayar 
Patricia L. Lakin-Thomas

Patricia Martinkova

Patrícia Raquel Vieira Sousa

Patricia Sánchez-González

Patricia Takako Endo

Patricia Vazquez

Patricio Bulic

Patricio Catalan

Patricio Colmegna

Patricio Domingues

Patricio Ordaz

Patrick Augustin

Patrick Callet

Patrick D. Kumavor

Patrick Degenaar

Patrick Erik Bradley

Patrick Fairclough

Patrick Kwong

Patrick Mounaix

Patrick Paitz

Patrick Seeling

Patrick Steglich

Patrick Stegmann

Patrick Van Torre

Patrick Wong

Patrik Flegner

Patrik Grznár

Patrik Richnák

Patrizia Grifoni

Patrizia Livreri

Patrizia Vizza

Patryk Ziolkowski

Patxi Casas

Pau Climent-Pérez

Paul Annus

Paul Bogdan

Paul Celicourt

Paul Davidsson

Paul E. Rapp

Paul E. Summers

Paul Farago

Paul Geladi

Paul Goodrum

Paul Havig

Paul Hemeren

Paul Kainen

Paul Krause

Paul Lik Hang Lee

Paul N. Whitehead

Paul Roberts

Paul Ruffin
Paul Schwarzbach

Paul Sestraș

Paul Sotiriadis

Paul Tavolato

Paul Thevenon

Paul Tucan

Paul Watson

Paula Bajdor

Paula Costa Castro

Paula Lamo

Paula Ruiz-Hincapie

Paula Sampaio

Paula Viana

Pauliina Salmi

Paulina Latko-Durałek

Paulina Lewińska

Paulina Trybek

Paulius Ruzgys

Paulius Skačkauskas

Paulo A. Raymundo-Pereira

Paulo Alexandre S. Armada Da Silva

Paulo Caldas

Paulo Coelho

Paulo De Mattos Neto

Paulo Dias

Paulo E. Cruvinel

Paulo Eduardo Teodoro

Paulo Gomes Da Costa

Paulo Goncalves

Paulo Jorge Palma

Paulo Moisés Costa

Paulo Montezuma

Paulo Montezuma-Carvalho

Paulo Reis

Paulo Ribeiro

Paulo Ribeiro Lins Júnior

Paulo Victor R. Ferreira

Pavan Poudel

Pavan Tankasala

Pavel Atanasoae

Pavel Fiala

Pavel Hrnčiřík

Pavel Karlovsky

Pavel Koštial

Pavel Kovář

Pavel Krasnov

Pavel Lafata

Pavel Loskot

Pavel Melnikov

Pavel Neužil

Pavel Otřísal 
Pavel Raška

Pavel Ripka

Pavel Segec

Pavel Stefanovič

Pavel Tofel

Pavel Turalchuk

Pavel V. Matrenin

Pavel Zemcik

Pavle Skočir

Pavlos Papadopoulos

Pavol Helebrandt

Pavol Kajánek

Pavol Kurdel

Pavol Lengvarský

Pavol Lipovský

Pawan Pathak

Pawel Andrzej Laski

Paweł Borkowski

Paweł Burdziakowski

Paweł Dymora

Paweł Gać

Paweł Grzegorz Gilewski

Paweł Herbin

Paweł J. Swornowski

Paweł Kaniewski

Pawel Kasprowski

Pawel Kleczynski

Paweł Komada

Paweł Kostyła

Paweł Krzaczek

Paweł Kudela

Paweł Ligęza

Pawel Linek

Pawel Macek

Pawel Madejczyk

Pawel Marc

Paweł Mazurek

Paweł Modrzyński

Paweł Olejnik

Pawel Pawlik

Paweł Pawłowski

Paweł Piotrowski

Paweł Piskur

Paweł Popielski

Paweł Romanowicz

Paweł Rosa

Pawel Rzucidlo

Paweł S. Wróbel

Paweł Sikora

Pawel Skokowski

Paweł Skruch
Paweł Sutowski

Paweł Szabelski

Pawel Tysiac

Paweł Wierzba

Paweł Zalewski

Paweł Ziolkowski

Paweł Zmarzły

Payam Shams Ghahfarokhi

Pay-Liam Lin

Paz Morer-Camo

Pedro A. Morais

Pedro Beirão

Pedro Bertemes-Filho

Pedro Branco

Pedro C. Junior Oliveira

Pedro Carmona

Pedro Casau

Pedro Castillejo Parrilla

Pedro Cruz

Pedro D. Frazão Correia

Pedro Dinis Loureiro Salgueiro

Pedro E. Lopez-De-Teruel

Pedro Encarnação

Pedro Faia

Pedro Fernández Álvarez

Pedro Fonseca

Pedro Forte

Pedro Henrique Lopes Silva

Pedro J. Sanchez-Cuevas

Pedro Javier Gamez Montero

Pedro Javier Herrera

Pedro M. B. Torres

Pedro Manuel Calas Lopes Pacheco

Pedro Mateus

Pedro Mêda

Pedro Melo

Pedro Mendonça Dos Santos

Pedro Miguel Alves Ribeiro Correia

Pedro Miguel Ortega-Cabezas

Pedro Moreno-Sanchez

Pedro O. Rossel

Pedro Oliveira Conceição Junior

Pedro Orgeira-Crespo

Pedro P. B. Beaklini

Pedro Pablo Garrido Abenza

Pedro Palos-Sánchez

Pedro Pinho

Pedro Piqueras

Pedro Ribeiro

Pedro Valdivia-Moral

Pedro Valero-Lara 
Peichang Zhang

Peichao Gao

Pei-Chen Kuan

Pei-Chi Shao

Peifeng Ma

Peiheng Zhou

Pei-Huang Diao

Peiliang $\mathrm{Xu}$

Peilong Hong

Peipei Liu

Peipei Wang

Peiyuan Zhou

Peiyun Zhang

Pekka Pirinen

Pelagia Gawronek

Peng Chen

Peng Fang

Peng Fu

Peng $\mathrm{Hu}$

Peng Li

Peng Liu

Peng Miao

Peng Qi

Peng Shao

Peng Song

Peng Tao

Peng Un Mak

Peng Wang

Peng Wu

Peng Yin

Peng Yu

Peng Zeng

Pengcheng Jiao

Pengcheng Liu

Pengfei Liu

Pengfei Tian

Pengfei Wang

Pengfei Zhang

Penghui Wang

Pengmin Pan

Pengpeng $\mathrm{Hu}$

Pengwen Xiong

Pengxiang Zhao

Pengyu Lv

Pengyuan Zhou

Pensieri Sara

Pentti Nieminen

Peppino Fazio

Per Håkan Lundow

Per Nordblad

Pere Vila Talleda
Pericle Perazzo

Perkowski Marek

Pero Vidan

Petar Radanliev

Peter A Lieberzeit

Peter Adam Hoeher

Peter Beek

Peter Biermann

Péter Böröcz

Peter Brida

Peter Centen

Peter Chong

Peter Cornillon

Peter Dragic

Péter Földesy

Peter Gege

Peter Hauser

Peter Hofmann

Peter Hubinsky

Peter J. Glynn

Peter Kaššay

Peter Kaul

Peter Kazanzides

Péter Kiss

Peter König

Peter Kostal

Péter Kovács

Peter L. Stavinoha

Peter Leary

Peter Maroti

Peter Meerwald-Stadler

Peter Mikulecký

Peter Offermans

Peter Ott

Peter Perešíni

Peter Pocta

Peter Rolfe

Peter Rössler

Peter Ryan

Peter Sarcevic

Peter Schulze Lammers

Peter Sevenich

Peter Sinčák

Peter Stütz

Peter Su

Péter Szolgay

Peter Teunissen

Peter Trúchly

Peter Veltink

Peter Wanke

Peter Williams 
Peter Wouters

Peter Zolliker

Peteris Apse-apsitis

Petr Beneš

Petr Boriskov

Petr Bouchner

Petr Červa

Petr Dolezel

Petr Eliáš

Petr Hájek

Petr Hlubina

Petr Hubacek

Petr Hurtík

Petr Koudelka

Petr Lehner

Petr Marcian

Petr Marcon

Petr Mlýnek

Petr Sedlák

Petr Skládal

Petr Štěpán

Petr Vachata

Petr Zhilyaev

Petra Đurović

Petra Jansen

Petre Daniel Matasaru

Petre Lameski

Petri Rönnholm

Petrică C. Pop

Petros Bithas

Petros Koutsovitis

Petros Pistofidis

Petros S. Bithas

Petru Ghenuche

Petrucio Ricardo Tavares De Medeiros

Pham Dinh Ba

Phani Sankar Nidadavolu

Philip Branch

Philip Chadwick

Philip Fuchs

Philip J. Basford

Philip Leroux

Philip Moore

Philipp Del Hougne

Philipp Gulde

Philipp M. Scholl

Philipp M. Sieberg

Philipp Terhörst

Philipp Wojaczek

Philippe Christol

Philippe Gueguen
Philippe Ravier

Phillip Bedggood

Phillip Millar

Phong B. Dao

Phuc Nguyen

Phuong T. Nguyen

Pi-Chung Wang

Pier Carlo Berri

Pier Giuseppe Anselma

Pier Matteo Barone

Pier Nicola Sergi

Pierangela Samarati

Piercarlo Dondi

Pierfrancesco Foglia

Pierluigi Belli

Pierluigi Ritrovato

Pierpaolo Loreti

Pierpaolo Palumbo

Pierre Boulanger

Pierre Exertier

Pierre Grangeat

Pierre Lacroix

Pierre Marcotte

Pierre Marechal

Pierre Merdrignac

Pierre-Edouard Portier

Pierre-jean Bouvet

Pierre-Paul Vidal

Pierre-Yves Collart-Dutilleul

Pierre-Yves De Müllenheim

Pierre-Yves Le Bas

Pieter Bonte

Pieter Monsieurs

Pieter Vandervoort

Pietro Aricò

Pietro Boccadoro

Pietro Burrascano

Pietro Cacialli

Pietro Catania

Pietro Monforte

Pietro Nannipieri

Pietro Piazzolla

Pietro Picerno

Pi-Guey Su

Pilar Cea Mingueza

Pilar García-Díaz

Pilar Jiménez

Pilar Manzanares-Lopez

Pilar Marin

Pilar Martín-Martín

Pilar Pérez-Ros 
Pin Lv

Pinaki Pal

Ping Lu

Ping Luo

Ping Wang

Ping Xie

Pingan Peng

Ping-Hui Lee

Pingjie Huang

Pingwei Liu

Pingyi Fan

Piotr Artiemjew

Piotr Bońkowski

Piotr Borkowski

Piotr Borowik

Piotr Cheluszka

Piotr Chrostowski

Piotr Cofta

Piotr Czech

Piotr Derugo

Piotr Drozdowski

Piotr Dulian

Piotr Dziurzanski

Piotr Fabian

Piotr Gauden

Piotr Gawda

Piotr Gazda

Piotr Gębara

Piotr Gierlak

Piotr Gołębiowski

Piotr Herbut

Piotr J. Durka

Piotr Jankowski-Mihułowicz

Piotr Jaworski

Piotr Kacejko

Piotr Kiedrowski

Piotr Kiełczyński

Piotr Kolasiński

Piotr Korbel

Piotr Krauze

Piotr Kurgan

Piotr Lesiak

Piotr Luliński

Piotr Maj

Piotr Markowski

Piotr Michalak

Piotr Miller

Piotr Miluski

Piotr Mitkowski

Piotr Morasiewicz

Piotr Napieralski
Piotr Nawrocki

Piotr Pałka

Piotr Piotr Lipiński

Piotr Porwik

Piotr Poznanski

Piotr Regulski

Piotr S. Szczepaniak

Piotr Sauer

Piotr Sawicki

Piotr Skrzypczyński

Piotr Sobotka

Piotr Stawicki

Piotr Tomczuk

Piotr Wisniowski

Piotr Wodarski

Piotr Wrobel

Piotr Zielinski

Pires Ivan

Piyapong Janmaimool

Piyush Yadav

Plácido Rogerio Pinheiro

Po-Chang Hsu

Po-Cheng Chang

Po-Chin Yang

Pohl László

Pol Henarejos

Polina P. Kuzhir

Polona Sprajc

Polyzois Soumplis

Poopalasingam Sivakumar

Po-Ruey Lei

Posen Lee

Poushali Das

Po-Wen Chi

Prabal Barua

Prabhuraj D. Venkatraman

Pradeeban Kathiravelu

Pradeep R. Varadwaj

Pradeep Sharma

Pradip Das

Prajowal Manandhar

Prakash Duraisamy

Prakash Gangadaran

Pramod K. B. Rangaiah

Pranav A. Bhounsule

Praneel Chand

Prashant Pandey

Prashanth Kond Gokuldoss

Prashanth Konda Gokuldoss

Prashnna Gyawali

Prasit Cholamjiak 
Prasun Kumar Nayak

Prateek M. Shrestha

Prateek Negi

Prateek Verma

Pratik Samant

Pratikkumar Desai

Pratima Labroo

Pratip Rana

Pratiti Bhadra

Pratool Bharti

Praveen Damacharla

Praveen Kumar Donta

Prem Chandra Pandey

Prem Kumar Chaurasiya

Premysl Hudec

Prince Waqas

Priscila M. Kosaka

Pritam Bose

Pritish Chakravarty

Priyabrata Pradhan

Priyadarsi Nanda

Priyanka Karnati

Priyanka Singh

Prodromos-Vasileios Mekikis

Proloy Deb

Prottoy Hasan

Przemyslaw Falkowski-Gilski

Przemysław Głomb

Przemysław Głowacki

Przemyslaw Herman

Przemysław Klapa

Przemysław Kuras

Przemysław Lisiński

Przemysław Mazurek

Przemysław Miąsik

Przemysław Podulka

Przemysław Przygodziński

Przemyslaw Ptak

Przemysław Wachulak

Pui-Chuen Hui

Pukar Maharjan

Puneet Mishra

Puran Pandey

Puren Ouyang

Pyke Tin

Pyo-Woong Son

Qaisar Abbas

Qaisar Abbass

Qaiser Riaz

Qammer H. Abbasi

Qasem Abu Al-Haija
Qasim Ahmed

Qi Liu

Qi Wang

Qi Zhang

Qi'ang Wang

Qian Du

Qian Feng

Qian Huang

Qian Li

Qian Sun

Qian Wang

Qian Yang

Qiancheng Zhao

Qiang Duan

Qiang Liu

Qiang Lv

Qiang Shen

Qiang Tang

Qiang Wu

Qianguo Xing

Qiangxian Huang

Qiangyu Zeng

Qiangyuan Zhu

Qianhong Chen

Qianru Jin

Qiao Li

Qibo Deng

Qibo Feng

Qican Zhang

Qichang Mei

Qichong Zhang

Qiguo Rong

Qihang Qiu

Qiji Ze

Qijin Chen

Qijin Huang

Qijin Zhang

Qijing Lin

Qilin Hua

Qimei Cui

Qing (Ken) Yang

Qing Chang

Qing Lu

Qing Xue

Qing Yang

Qing Zhang

Qingdong Ou

Qingfeng Liu

Qingguo Du

Qingguo Li

Qinghe Zheng 
Qinghua Huang

Qingjun Zhang

Qinglan Ding

Qingmin $\mathrm{Yu}$

Qingqi Pei

Qingshan She

Qingsong Wang

Qingtian Deng

Qingwen Han

Qingxi Zeng

Qingxu Deng

Qingyang Wei

Qingyong Li

Qingyu Cui

Qingyun Du

Qingzhou Mao

Qinjun Wang

Qinpei Zhao

Qiong Ran

Qiong Wang

Qiongfeng Shi

Qipei Mei

Qitao Tan

Qiu Jun

Qiuguo Zhu

Qiuliyang Yu

Qiuming Zhu

Qiuping Jiang

Qiushi Fu

Qiwen Qiu

Qiyao Huang

Qizhen Sun

Qizhi Xu

Qonita Kurnia Anjani

Quan Chai

Quan-De Wang

Quang Ha

Quang Hung Do

Quang Hung Tran

Quanqin Shao

Quanying $\mathrm{Wu}$

Quetzalcoatl Cruz Hernández-Escobedo

Qun Sun

Qunsheng Cao

Quoc-Anh Tran

Quoc-Hung Phan

Quoc-Thong Nguyen

Qutaiba Alasad

Quyuan Luo

R. Chad Webb

R. Dhaya
R. Jacob Baker

R. Ranjith Premasiri

R. Simon Sherratt

Ra'ed Malallah

Rabab Benotsmane

Rabie A. Ramadan

Rabih Younes

Raby Hamadi

Rachel Vitali

Rachel Zuanon

Rachid Benouini

Rachid Nedjai

Rachid Saadane

Rachna Valvani

Radek Šmíd

Radek Strnad

Radhya Sahal

Radim Burget

Radka Nacheva

Radoslav Tomović

Radoslav Vargic

Radosław Belka

Radosław Cioć

Radosław Klimek

Radosław Miśkiewicz

Radosław Podsiadły

Radosław Pomećko

Radostina A. Angelova

Radovan Holubek

Radu Ciorap

Radu Coliban

Radu Comes

Radu Gabriel Bozomitu

Radu Hobincu

Radu Radescu

Radu Tarca

Radu Tudor Ionescu

Radu Vacareanu

Raed Abd-Alhameed

Rafael Abrantes Penchel

Rafael Aguilar-Gonzalez

Rafael Alvarez

Rafael Asorey-Cacheda

Rafael Berlanga-Llavori

Rafael C. Cardoso

Rafael Casado

Rafael Cisneros-Magaña

Rafael Furlan De Oliveira

Rafael Gonzalez-Landaeta

Rafael Kunst

Rafael Martínez Peláez 
Rafael Messias Martins

Rafael Molina

Rafael Morales Herrera

Rafael Pastor Vargas

Rafael Perazzo Barbosa Mota

Rafael Perez Del Real

Rafael Pires De Lima

Rafael Radkowski

Rafael Ravina-Ripoll

Rafael Raya López

Rafael Santos

Rafael Timóteo De Sousa Júnior

Rafael Vargas-Bernal

Rafal Doroz

Rafał Dreżewski

Rafał Gołębski

Rafał Grądzki

Rafał Grzejda

Rafał J. Doniec

Rafał Kaźmierczak

Rafał Kowalik

Rafal Przesmycki

Rafał Sieńko

Rafał Szukiewicz

Rafał Wojszczyk

Raffaele Carli

Raffaele Gravina

Raffaele Martorana

Raffaele Ricco

Raffaele Salerno

Raffaele Scurati

Raffaele Velotta

Raffaele Zinno

Rafik Hamza

Ragab El Sehiemy

Rahim Rahmani

Rahman Ali

Rahul Datta

Rahul Gomes

Rahul Mourya

Rahul Reddy Nadikattu

Raihan Ul Islam

Raimon Casanova

Raimondas Pomarnacki

Raimondas Šadzevičius

Raimund Kirner

Raimundo Jiménez Ballesta

Raine Viitala

Rainer Falk

Rainer Tutsch

Raivo Stern
Raj K. GC

Raj Kumar

Raja Balaguru

Raja Nagisetty

Rajagopalan Thiruvengadathan

Rajalekshmi C. Pillai

Rajan Kadel

Rajani Singh

Rajendra Dhakal

Rajendra N. Bhadane

Rajesh Kumar

Rajesh Kumar Tripathy

Rajeshwara Chary Sriramadasu

Raji Ghawi

Rajkumar Saini

Raju Aedla

Raju Chetty

Ralf Bergmann

Ralf Fabian

Ralf Lübben

Ralf Moura

Ralf Stetter

Ralf Ziebold

Ralu Divan

Ram K. Mazumder

Ram Kumar Selvaraju

Ram M. Narayanan

Ram Narayanan

Rama Sashank Madhurapantula

Ramachandran Kasu

Ramanaskanda Braveenth

Ramasamy Mouli

Rameez Asif

Ramesh Nepal

Ramez Abdallah

Rami Ahmad El-Nabulsi

Rami Cohen

Rami Mustafa A Mohammad

Rami Zewail

Ramin Banan Sadeghian

Ramin Ghiasi

Ramin Rahmani

Ramin Yahyapour

Ramiro S. Barbosa

Ramiro Velázquez

Ramon Agüero

Ramón Alberto Mollineda Cárdenas

Ramón Alcarria

Ramon Antonio Rodriges Zalipynis

Ramon Aparicio-Pardo

Ramón Barber 
Ramon Casanella

Ramón De La Rosa

Ramon Dos Reis Fontes

Ramon Eritja

Ramon Fontes

Ramon García Hernández

Ramón González-Camarena

Ramón Moreno

Ramon Parra-Michel

Ramon Villarino

Ramona Cimpoesu

Ramona Galatus

Ramūnas Česnavičius

Ran Chen

Ran Li

Ran Liao

Ran Liu

Ran Novitsky Nof

Rana Zia Ur Rehman

Randolph Kirk

Ranesh Kumar Naha

Rangeet Mitra

Ranil Gurusinghe

Ranjit Shrestha

Ranjith Liyanapathirana

Ranko Heindl

Ranko Zotovic Stanisic

Ranniery Maia

Rao Fu

Raouf Senhadji-Navarro

Raoul Nigmatullin

Raoul Reiser

Raphael Deltombe

Raphael Horvath

Raphaël Khoury

Raphaël Turcotte

Raphael Vallon

Raphael Zaccone

Raquel Cama-Moncunill

Raquel Cervigón

Raquel Conceição

Raquel De Los Reyes

Raquel Leirós-Rodríguez

Raquel López Heredero

Raquel Martínez España

Raquel Mayordomo

Raquel Montes

Raquel Sanchis

Rasa Pauliukaite

Rashid Ali

Rashmi Sharan Sinha
Rasmus Rettig

Rasmus Rivinius

Rasoul Shalchi Alishah

Rastislav Pirnik

Ratko Grbić

Raúl Alberto Ponce Rodríguez

Raúl Alcaraz Martínez

Raul Ceretta Nunes

Raul Cristian Roman

Raul Fernandez-Garcia

Raul Fizesan

Raul Igual Catalan

Raúl Jiménez-Naharro

Raúl Marticorena-Sánchez

Raúl Montoliu

Raul Onet

Raúl Parada

Raúl Parada Medina

Raul Rabinovici

Raúl Suárez

Raul-Cristian Roman

Ravi Ancil Persad

Ravi Kumar Cheedarala

Ravi Reddy Manumachu

Ravil Muhamedyev

Raviraj Thakur

Rawaz Kurda

Rawil Fakhrullin

Raymond K. Zhao

Raymundo Buenrostro-Mariscal

Rayya A. Al-Balushi

Rayyan Manwar

Ray-Yeng Yang

Raza Qazi

Razaque Abdul

Razvan Boboc

Razvan Bogdan

Razvan Craciunescu

Razvan D. Tamas

Răzvan Gabriel Boboc

Razvan Ioan Pacurar

Răzvan Rughiniș

Razvan Serbu

Rebeca Jiménez-Pérez

Rebeca P. Díaz Redondo

Rebecca A. States

Redha Taiar

Redmond R. Shamshiri

Reed Gurchiek

Reem Hanna

Regine Frank 
Rehan Siddiqui

Rehmat Ullah

Reik Donner

Reinaldo Martinez Palhares

Reiner Jedermann

Reiner Kriesten

Reinhold Herschel

Rekha Guchhait

Rem Collier

Remedios Lopez-Liria

Remigiusz Bąchor

Remigiusz Rajewski

Remous-Aris Koutsiamanis

Remus Brad

Remus Pravalie

Ren Cheng Jin

Ren Cooper

Renaldas Urniezius

Renata Borovica-Gajic

Renata Činčikaitè

Renata Dondajewska-Pielka

Renata Gržić

Renata Karpicz

Renata Kelly Mendes

Renata Lopes Rosa

Renata Markowska

Renata Sisto

Renato César Dos Santos

Renato Giacomini

Renato Henriques

Renato Miyagusuku

Renato Sobral Monteiro

Rencheng Song

Rendy Thamrin

René M. Rossi

Reneta Barneva

Ren-Hung Hwang

Renlong Hang

Renxin Wang

Renyuan Qin

Renzo Capitani

Reuben Burch

Revannath Nikam

Rex Tan

Reza Shahbazian

Reza Shahidi

Reza Tafreshi

Rhodri Smith

Riad Mokadem

Ricardo Alexandre Reinaldo De Moraes

Ricardo Ambrocio Ramirez-Mendoza
Ricardo Augusto Rabelo Oliveira

Ricardo Carmona-Galán

Ricardo Cordoba

Ricardo Dalagnol

Ricardo Escobar-Jiménez

Ricardo Ferreira

Ricardo Jimenez

Ricardo Kamikawachi

Ricardo Oliveira

Ricardo Perera

Ricardo Piedrahita

Ricardo Rabelo

Ricardo Ron-Angevin

Ricardo Santos

Ricardo Vardasca

Ricardo Vergaz

Ricardo Vinuesa

Ricardo Zednik

Riccardo Bernardini

Riccardo Carotenuto

Riccardo Colella

Riccardo De Benedictis

Riccardo De Gaudenzi

Riccardo Giubilato

Riccardo Mandriota

Riccardo Miscioscia

Riccardo Monica

Riccardo Ortale

Riccardo Palamà

Riccardo Zucca

Richard C. Dowell

Richard Demo Souza

Richard Dewey

Richard Dvořák

Richard Funk

Richard G. Trohman

Richard Godinez Tello

Richard Greenwald

Richard H. Sandler

Richard Hauer

Richard Hopper

Richard J Sheridan

Richard Klemm

Richard Segall

Richard Souza

Richard W. Bohannon

Richard Watson

Rick Sturdivant

Rick Y.C. Kwan

Ridwan Alam

Riëm El Tahry 
Rih Teng Wu

Riheng Jia

Riichi Murayama

Rijuta Ganesh

Rikiya Takeuchi

Rikke Gade

Riko Šafarič

Rimas Janeliukstis

Rinkaj Goyal

Rishikesh Pandey

Rita Bacelar Figueira

Rita Catalina Aquino Caregnato

Rita Figueira

Rita Girão Silva

Rita M. Kiss

Rita Stagni

Ritesh Chimoriya

Ritesh Kumar Singh

Ritesh Vyas

Ritu Malik

Rituparna Chaki

Rizwan Ahmed Khan

Rizwan Ali Naqvi

Rizwan Aslam Butt

Roald M. Tiggelaar

Roald Otnes

Roana De Oliveira Hansen

Rob F. Remis

Rob Wijnhoven

Robert A. Cohen

Robert A. Convissar

Robert A. Mickler

Robert Barański

Robert Bestak

Robert Burduk

Robert Castilla

Robert Catena

Robert Chodorek

Robert Crowther

Robert Czerwinski

Robert Demeter

Robert Dickinson

Robert Dyja

Robert Frischholz

Robert Gabriel Lupu

Robert Henderson

Robert Konrad Szymczak

Robert Krewinkel

Robert Kubina

Robert LiKamWa

Robert M. Nowak
Robert Milewski

Robert Mingesz

Robert Mroczyński

Robert Newcomb

Robert Nowak

Robert Nuster

Robert Oleniacz

Róbert Olšiak

Robert P. Johnson

Robert Panowicz

Robert Pasken

Robert Philipp

Robert R. Sattarov

Robert Roczniok

Robert Rusinek

Robert S. Bradley

Robert Schmitt

Robert Sitnik

Robert Soto

Robert Sousa

Robert Szczepanek

Robert T. Menzies

Robert Tomkowski

Robert Ulewicz

Robert Watson

Robert Weber

Robert Weser

Robert Župan

Roberta Calmo

Roberta D'Aurelio

Roberta Gasparro

Roberta Perego

Robert-Alexandru Dobre

Robertas Damaševičius

Roberto Alejo Eleuterio

Roberto Bellasio

Roberto Bernasconi

Roberto Cannataro

Roberto Carlos Ambrosio-lazaro

Roberto Casado-Vara

Roberto Celio Limao De Oliveira

Roberto Colasanti

Roberto Corizzo

Roberto Corvaja

Roberto Di Marco

Roberto Francescon

Roberto G. Citarella

Roberto G. Valenti

Roberto Giovanni Ramírez-Chavarría

Roberto Li Voti

Roberto López-Valcarce 
Roberto Macaluso

Roberto Merino-Martinez

Roberto Miorelli

Roberto Nebuloni

Roberto Opromolla

Roberto Orosei

Roberto Paiano

Roberto Paolesse

Roberto Peron

Roberto Pierdicca

Roberto Raffaeli

Roberto Romeo

Roberto S. Netto

Roberto Sabatini

Roberto Saia

Roberto Tagliaferri

Roberto Teghil

Roberto Teti

Roberto Valentino

Roberto Vincenti Gatti

Robin Dobos

Robin Gerzaguet

Robyn Jones

Roc Berenguer

Roc Meseguer

Rocco Alaggio

Rocco Citroni

Rocco Fazzolari

Rocco Furferi

Rocco Haase

Rocio B. Dominguez

Rocio Canovas Martinez

Rocío Josefina Pérez De Prado

Rocio Sanchez-Montero

Rodica Elena Ionescu

Rodica Holonec

Rodica Voicu

Rodica-Mariana Ion

Rodolfo Haber

Rodolfo Meneguette

Rodolfo Miranda Pereira

Rodolfo Reda

Rodolphe Heyd

Rodrigo A. A. Munoz

Rodrigo Braga

Rodrigo Colnago Contreras

Rodrigo Filev Maia

Rodrigo Gomes Da Rosa

Rodrigo Gonzalez

Rodrigo Martín De San Agustín

Rodrigo Oyonarte
Rodrigo Righi

Rodrigo Rocha Silva

Rodrigo Salas

Rodrigo Sanches Miani

Rodrigo Santos

Roemi Fernandez

Rogelio Bustamante-Bello

Rogelio De J. Portillo-Vélez

Rogelio Gónzalez-Gónzalez

Rogelio Hasimoto-Beltran

Roger Bilham

Roger C. Lowe

Roger M. Krzyżewski

Roger Pissard-Gibollet

Roger Resmini

Rogerio Atem De Carvalho

Rogério Dionísio

Rogerio Manica

Rohan Nagare

Rohan Soman

Rohit Mishra

Rohit Verma

Roi Méndez

Roja Esmaeeli

Rok Dolenec

Rok Vrabič

Roland Bejjani

Roland Bouffanais

Roland Chapuis

Roland Valcke

Roland Zügner

Rolando Herrero

Rolf Becker

Romain Jacob

Roman A. Zakoldaev

Román Alcides Lara-Cueva

Roman Bauer

Roman Danel

Roman Fediuk

Roman Fedorenko

Roman Forke

Roman Grill

Roman Holovchak

Roman Kolcun

Roman Krzanowski

Roman Kubacki

Roman Kusche

Roman Major

Roman Maršálek

Roman Regulski

Roman Rousseau 
Roman Shults

Roman Skidanov

Roman Sotner

Roman Szewczyk

Roman Trach

Roman Trochimczuk

Roman Tsvetkov

Roman V. Klyuev

Roman Viter

Roman Wyrzykowski

Roman Y. Pishchalnikov

Romana Schirhagl

Romolo Marcelli

Romuald Boucheron

Romualdas Baušys

Romualdas Navickas

Romulus Costache

Romulus Terebes

Ronald Leeper

Ronald Roberts

Ronan Paugam

Rone Ilídio Da Silva

Roneel Sharan

Rong Ran

Rong Yin

Rong-Ho Lee

Rong-hua Huan

Ronghua Ma

Ronghui Zhan

Rongpeng Li

Rongrong Liu

Rongyong Huang

Ronnie O. Serfa Juan

Roohallah Alizadehsani

Roope Raisamo

Roozbeh Abedini-Nassab

Roozbeh Atri

Roque A. Osornio Ríos

Rory Cooper

Rory O'Sullivan

Rosa Rio-Belver

Rosalba Gaudiuso

Rosalinda Inguanta

Rosalyn L. Putland

Rosalynn Nankya

Rosamaria Salvatori

Rosana Alves Dias

Rosanna Viglialoro

Rosaria Rucco

Rosario Arjona

Rosario Fedele
Rosario García-Giménez

Rosario Oliva

Rosario Porras-Aguilar

Rosario Schiano Lo Moriello

Roshan Dsouza

Roshan Joseph

Roshan Khadka

Rosilah Hassan

Rosolino Vaiana

Ross Cheriton

Ross Clark

Ross Darnell

Ross N. Gillanders

Rostam Affendi Hamzah

Roufei Chen

Roumen Kirov

Roussin Lontio Fomekong

Roxana Radvan

Roxana-Mariana Beiu

Roxanne Radpour

Roy B. V. B. Simorangkir

Roya Nasimi

Ruan Delgado Gomes

Rubén Carlos Acevedo

Rubén Martín-Clemente

Ruben Medina

Ruben Pauwels

Rubén Picó Vila

Rubén San-Segundo

Ruber Hernández-García

Rucha Deshpande

Ruchire Eranga Wijesinghe

Ruchire Wijesinghe

Rüdiger Gerdes

Rüdiger Rupp

Rudinei Goularte

Rudnei Dias Da Cunha

Rudnik Katarzyna

Rudolf Janos

Rudolf Urban

Rudolf Verdaasdonk

Ruei-Sung Yu

Rueter Dirk

Ruey-Ching Twu

Ruey-Kai Sheu

Ruggero Lanotte

Ruggero Reggiannini

Rugved Likhite

Ruhollah Taghizadeh-Mehrjardi

Rui Antunes

Rui Bai 
Rui Bernardes

Rui Campos

Rui Costa Martins

Rui Dilão

Rui Dinis

Rui Dong

Rui Fonseca-Pinto

Rui Li

Rui Lin

Rui Min

Rui Pedro Julião

Rui Pedro Lopes

Rui Silva

Rui Silva Moreira

Rui Sun

Rui Vasco Silva

Rui Yang

Rui Yuan

Rui Zhang

Rui Zhao

Ruifeng Li

Ruihai Dong

Ruijie Wang

Ruijun Li

Ruinian Jiang

Ruiqing Shen

Ruishu F. Wright

Ruisong Han

Ruitao Yang

Ruixiang Bai

Ruiyu Liang

Rujie He

Rukhsana Ruby

Ruman Banerjee

Rune Schlanbusch

Runmin Cong

Runqi Chai

Runqiang Zeng

Runze Li

Ruocan Qian

Ruochen Huang

Ruochen $\mathrm{Lu}$

Ruopeng Sun

Ruoxi Sun

Ruoxiu Xiao

Ruoyu Su

Rupesh Shrestha

Ruriko Hatada

Ruriko Yoshida

Rushi Lan

Rushit Dave
Ruslan E. Asfin

Russell Farrugia

Russo Paolo

Rustam Khabibullin

Rüstem Keçili

Ruszczak Bogdan

Rūta Gerasimaitè

Rutger Osterthun

Ruth MacKay

Ruth Shinar

Ruud G. J. Meulenbroek

Ruxandra F. Olimid

Ruxandra Stoean

Ruxandra Tapu

Ruya Xiao

Ryad Bouzouidja

Ryad Zemouri

Ryan Chapman

Ryan Gibson

Ryan Mattfeld

Ryan Niemeier

Ryan Ramirez

Ryan T. White

Ryan Terrien

Rymantas Jonas Kažys

Rymarczyk Tomasz

Ryo Natsuaki

Ryohei Misumi

Ryoji Kurita

Ryoma Morigaki

Ryou Ohsawa

Ryou Tanaka

Ryszard Jachowicz

Ryszard Pawlak

Ryuichi Ohta

Ryuji Hamamoto

Ryuki Sato

S. Zohreh Homayounfar

S.M. Sohel Mahmud

Sabato Mellone

Sabina Licen

Sabina Maraffi

Sabina Merlo

Sabina Szymoniak

Sabino Maggi

Sabita Panicker

Sabri Allani

Sabrina Demarie

Sabrina Di Masi

Sabrina Leo

Sabrine Kheriji 
Sabyasachi Mondal

Sachin Kumar

Sachin Navale

Sachin Sharma

Sachit Mahajan

Sadegh Samadi

Sadeq Saleh

Sadeque Reza Khan

Sadko Mandžuka

Sadok Rezig

Sae-Bae Napa

Saed Moradi

Saeed Hamood Alsamhi

Saeed Khaki

Saeed M. Bamatraf

Saeed M. Qaisar

Saeed Mahmoudpour

Saeed Mouloodi

Saeed Nosratabadi

Saeed Olyaee

Saeed Rehman

Saeed Shavvalpour

Saeid Niazmardi

Saeid Pourroostaei Ardakani

Sagar Jinachandran

Sagi Filin

Sagrario Martín-Aragón

Sahaj Saxena

Sahameddin Mahmoudi Kurdistani

Sahand Pirouzpanah

Sahraoui Dhelim

Sai G.S. Pai

Sai Huang

Sai Wei Yang

Said Benissa

Said Munir

Said Quqa

Saiful Bari

Saim Memon

Saima Qureshi

Saiqi Tian

Sajad Jamshidi

Sajad Mohamadzadeh

Sajad Sabzi

Sajeesh Kumar

Sajid Nisar

Sajjad Hussain

Sakdirat Kaewunruen

Sakib K. Pathan

Salah Khan

Salaheldin Elkatatny
Salam Al-Sabah

Salam Ibrahim

Salam Khamas

Salau Bello

Saleh Abujarad

Saleh Aly

Saleh Mohammad

Salicone Simona

Salik Ram Khanal

Salil Karipott

Salim Lamine

Sally McClean

Salma Elsayed

Salman Ahmadi-Asl

Salman Ali

Salman I. Siddiqui

Salua Hamaza

Salvador Alcaraz

Salvador Barrera Figueroa

Salvador Dueñas Carazo

Salvador J. Jaime

Salvador Mendoza-Acevedo

Salvador Moreno

Salvatore A. Pullano

Salvatore Carta

Salvatore Daniele

Salvatore Falanga Bolognesi

Salvatore Graziani

Salvatore Musumeci

Salvatore Ponte

Salvatore Sessa

Salvatore Surdo

Salvatore Tedesco

Salvina Gagliano

Salvo Marcuccio

Sam Amiri

Sam Lofland

Sam Reisenfeld

Samad Sepasgozar

Samadhan Bhaulal Patil

Saman Hedjazi

Samaneh Kouchaki

Samantha Dugelay

Sambor Guze

Sambuddha Ghosal

Sameer Chakravarthy Vedula

Sameera Wickramasinghe

Samer Kharroubi

Samer Khouri

Sami Barmada

Sami Myllymaa 
Sami Nikkonen

Sami Obaid

Samir Hachour

Samir Khatir

Samir Malakar

Samir Mustapha

Samira Briongos

Samira Naghdi

Samntha Macchi

Samo Stanič

Sampsa Vanhatalo

Samuel Agbroko

Samuel Deslauriers-Gauthier

Samuel Kofi Erskine

Samuel Lawman

Samuel Montejo Sánchez

Samuel Morillas Gomez

Samuel Ortega Sarmiento

Samuel Silva

Samuel X. Shi

Samuel Xavier-de-Souza

Samuele De Petris

Samuele Gelli

Samy Shaban

Sana Syed

Sana Ullah Jan

Sanath Kumar Sathyachandran

Sanathanan Muttikulangara

Sanaz Jarolmasjed

Sanaz Kavianpour

Sanaz Pournajaf

Sanaz Rahimi Moosavi

Sanbo Qin

Sandeep K. Chaudhuri

Sandeep K. Singhal

Sandeep Kumar

Sandeep Sharma

Sandeep Sony

Sander Oude Elberink

Sandhiran Patchay

Sandip Dutta

Sandor Frey

Sándor Szénási

Sándor Valkai

Sandra Bravo

Sandra Cano

Sandra Drusová

Sandra Dudley

Sandra Gama

Sandra García-Gallego

Sandra Grabowska
Sandra Mogo

Sandra Rodrigues

Sandra Roger

Sandro Da Silva Camargo

Sandro Fioretti

Sandy Cochran

Sanford Meek

Sang Hun Lee

Sang Hyun Lee

Sang Joon Park

Sang Jun Lee

Sang M. Won

Sang Won Bae

Sangchoon Kim

Sangdae Kim

Sangeet Saha

SangGon Lee

Sangho Bok

Sanghoon Lee

Sangita Das

Sangita Pal

Sang-Jae Kim

Sangjin Byun

Sang-Jin Choi

Sangjo Choi

Sangkil Kim

Sangman Moh

Sangmin An

Sangmin Lee

Sangrok Jin

Sangsoon Lim

Sang-Woo Seo

Sang-Yoon Chang

Sanislav Teodora

Sanja Mahovic Poljacek

Sanja Martinez

Sanja Radosavljevic

Sanjay Kumar

Sanjay Kumar Biswash

Sanjeev Arulampalam

Sanjeev Kumar Sagwal

Sanjeev Tambe

Sanju Gupta

Sanket Awate

Sanoj Rejinold

Sansit Patnaik

Sante Francesco Rende

Santhakumar Sampath

Santiago Cepeda

Santiago Felici-Castell

Santiago Gutiérrez 
Santiago Puente

Santiago Sánchez-Solano

Santo Orlando

Santos Sanchez-Cambronero

Santosh Kumar

Santosh Kumar Paidi

Santosh Pitla

Santosh Shrestha

Santosh Subedi

Santoso Wibowo

Saptarshi Mukherjee

Saptarshi Sarkar

Saptarshi Sengupta

Sara Casaccia

Sara Comai

Sara Díaz Cardell

Sara Ferreira

Sara Gonizzi Barsanti

Sara Jayousi

Sara Moccia

Sara Stančin

Sara Stolyarova

Sara Todeschini

Sara Tombelli

Sarah Azimi

Sarah Hubbard

Sarah R.B. King

Sarah Ringerud

Sarah Tonello

Saranraj Karuppuswami

Sarath Vijayakumar

Sarfaraz Adnan

Sarita Keski-Saari

Sarita Mazzini Bruschi

Sarthak Pathak

Sarunas Grigaliunas

Sarunas Meskinis

Saša Ćuković

Sasa Djuric

Sasa Mrdovic

Sasa Sladic

Sascha Eichstädt

Sascha Fleer

Sascha Preu

Saskia Kraft-Bermuth

Sasya Madhurantakam

Satheesh Chandran

Sathishkumar Samiappan

Sathiyaraj Kandhasamy

Sathwik S. Kasyap

Sathyachandran Sanath Kumar
Satish Mahadevan Srinivasan

Satoru Tsujii

Satoshi Kurihara

Satoshi Matsumoto

Satoshi Saga

Satoshi Tsuji

Satoshi Ueno

Satoshi Yamane

Satyabrata Aich

Satyendra Kumar Mishra

Saugat Bhattacharyya

Saul Delabrida

Saúl E. Pomares Hernández

Saúl Vallejos Calzada

Saulius Balevicius

Saulius Juodkazis

Saurabh Sahu

Saurabh Shukla

Saurabh Singh

Saurin Patel

Saverio Latorrata

Saverio Maietta

Savio Sciancalepore

Sawyer D. Campbell

Sayan Ganguly

Sayan Roy

Sayed Pouria Talebi

Saygin Abdikan

Sazzadur Chowdhury

Scott A. Trammell

Scott Adams

Scott E. Budge

Scott Thomas

Scott White

Se Dong Min

Seahwa Won

Sean Casey

Sean Elvidge

Sean McKeown

Sebania Libertino

Sebastia Galmes

Sebastian Bader

Sebastian Bähr

Sebastian Berhausen

Sebastian Böttcher

Sebastian Budzan

Sebastian Dahle

Sebastian Dudzik

Sebastián García Galán

Sebastian Głowiński

Sebastian Gutierrez 
Sebastian Hegler

Sebastian Hensel

Sebastian Kapp

Sebastian Kot

Sebastian Müller

Sebastian Ramacher

Sebastian Scheurer

Sebastian Schnaubelt

Sebastian Seidel

Sebastian Steinlechner

Sebastian Tomczak

Sebastian Troia

Sebastian Tschauner

Sebastian Wolf

Sebastian Zlotnik

Sebastiano Massaro

Sebastiano Milardo

Sébastien Combéfis

Sebastien Gaboury

Sebastien Gadal

Sébastien Lallechere

Sébastien Mouchet

Sebastijan Mrak

Sedig S. Agili

Sedighi Saeid

Seehwan Yoo

Sehwan Choi

Seid Koric

Seid Miad Zandavi

Seigo Ohno

Seiya Suzuki

Se-Jin Lee

Sejin Park

Sejung Yang

Sekchin Chang

Selda Oezkan

Selim Solmaz

Selma Rizvič

Sen Qiu

Sen Wang

Sen Yan

Senghan Yoo

Senol Piskin

Senthil Kumar Karuppannan

SenthilKumar Mohan

Seok Jae Chu

Seok-Cheol Kee

Seok-Ho Rhi

Seok-Hwan Jeong

Seok-Joo Koh

Seonah Lee
Seong Jung Kwon

Seong Soo A. An

Seongah Jeong

Seongchan Kim

Seong-Cheol Kim

Seong-Eun Moon

Seong-eun Yoo

Seong-Heum Kim

Seonghun Hoon Kim

Seong-Ik Han

Seongjin Lee

Seongju Chang

Seongkeun Park

Seong-Kyu Kim

Seongmin Heo

SeongMin $\mathrm{Oh}$

Seong-Tae Han

Seongwook Lee

Sepehr Alizadehsalehi

Sepideh Khoshnevis

Septimiu Lica

Septimiu Mischie

Serafeim Moustakidis

Serafin Lopez-Cuervo Medina

Serafín Moral-García

Serban Chivulescu

Serban Georgica Obreja

Serban Meza

Serena Dattola

Serge Tamari

Sergei A. Soldatenko

Sergei Chernyi

Sergei I. Badulin

Sergei Komogortsev

Sergei Kulinich

Sergei Piskunov

Sergei Popov

Sergei Savin

Sergejs Kodors

Sergey A. Butenkov

Sergey A. Shevchik

Sergey A. Stel'makh

Sergey Aleshin

Sergey Bezzateev

Sergey Gudoshnikov

Sergey I. Fomenko

Sergey K. Aityan

Sergey Kobtsev

Sergey Konovalov

Sergey Kornilov

Sergey Kucheryavskiy 
Sergey N. Shevtsov

Sergey Pavlovich Morozov

Sergey Pavlovich Osipov

Sergey Pirogov

Sergey S. Krasilnikov

Sergey Shevchenko

Sergey Shevtsov

Sergey Shoydin

Sergey Sokolov

Sergey V. Zavjalov

Sergey Vadimovich Anakhov

Sergey Vinogradov

Sergey Zavadskiy

Sergey Zhironkin

Sergieieva Kateryna Leoidivna

Sergii Babichev

Sergii Kushch

Sergio A. Araujo-Estrada

Sergio A. David

Sergio Calixto

Sergio Camiz

Sergio Carrato

Sergio De Salvatore

Sergio Dominguez

Sérgio Duarte Correia

Sergio Gonzalez-Sevilla

Sergio Hernandez

Sérgio I. Lopes

Sergio Ibarra Espinosa

Sergio L. Stevan, Jr.

Sergio López-García

Sergio Luis Suárez Gómez

Sergio M. O. Tavares

Sergio Martin-del-Campo

Sergio Montenegro

Sérgio Moro

Sergio Ruggieri

Sergio Silva Ribeiro

Sergio Torres

Sergio Valero

Sergiusz Łuczak

Sergiusz Patela

Serguei Primak

Serguei Stoukatch

Serhii Stepenko

Serioja Ovidiu Tatu

Seth H. Peterson

Seung Lee

Seung Yun

Seung-Bok Choi

Seungho Jung
Seung-Ho Lim

Seung-Hwan Bae

Seunghwan Lee

Seung-Ik Lee

Seungkyu Ryu

Seungmo Kim

Seung-Nam Kim

Seungryul Baek

Seungwon Song

Seungyub Lee

Sever-Gabriel Racz

Severino Munoz-Aguirre

Seyed Ali Cheraghi

Seyed Ali Ghahari

Seyed Ali Rezvani

Seyed Amir Hoseinitabatabaei

Seyed Hamidreza Aghay Kaboli

Seyed Mahdi Darroudi

Seyed Mohammad Ghoreyshi

Seyed Mostafa Mousavi Kahaki

Seyed Saeid Mohtasebi

Seyedabdollah Mirbozorgi

Seyedali Mirjalili

Seyed-Ali Sadegh-Zadeh

Seyedfakhreddin Nabavi

Seyedmohammadreza Heibati

Seyednima Khezr

Shabnam Jabari

Shabnam Sadeghi Esfahlani

Shaddrack Yaw Nusenu

Shah Nawaz Burokur

Shah Nazir

Shahab Eddin Jozdani

Shahab Haghayegh

Shahab Heshmati-alamdari

Shahab Pasha

Shahab Tayeb

Shahid Hussain

Shahinur Alam

Shahood Uz Zaman

Shahram Heydari

Shahram Moafipoor

Shahram Payandeh

Shahram Sattar

Shahzad Ashraf

Shaibal Barua

Shaila Afroj

Shailabh Kumar

Shailendra Rajput

Shakti P.C.

Shalei Song 
Shama Islam

Shamik Tiwari

Shan Jiang

Shancheng Cao

Shancheng Yan

Shangfei Song

Shangjie Ren

Shangran Xie

Shanhai Jin

Shankargouda Patil

Shanpu Shen

Shanq-jang Ruan

Shanshan Gu-Stoppel

Shanshan Wang

Shanshan Wei

Shantanu Pal

Shantonu Biswas

Shaocheng Luo

Shaocheng Qu

Shaochun Dong

Shaofang Gong

Shaohua Wan

Shaohua Wang

Shaohui Liu

Shaojie Qiao

Shaojing Fan

Shaojuan Luo

Shao-Ku Kao

Shaolin Zhang

Shaolong Kuang

Shaopeng $\mathrm{Hu}$

Shaopeng Wang

Shaoqing $\mathrm{Hu}$

Shao-Yi Hsia

Shaqfeh Mohammad

Sharief Oteafy

Sharifu Ura

Sharnil Pandya

Shashi Kant Bhatia

Shaukat Ali Mazari

Shaul Ozeri

Shaun M. Sharpe

Shaun West

Shawana Tabassum

Shawn M. Beaudette

Shayan Angizi

Shazali Osman

Sheeraz Ahmed

Shehzad Ashraf Chaudhry

Sheikh Akbar

Sheikh Mohammad Idrees
Sheikh Shanawaz Mostafa

Shekh Md Mahmudul Islam

Shelly Vishwakarma

Shen Liu

Shen Yin

Shen Ying

Sheng Fu

Sheng Lih Yeh

Sheng Tan

Sheng Wang

Sheng $\mathrm{Xu}$

Shengbo Chen

Shengbo Shan

Shengchang Lan

Sheng-Chi Liu

Sheng-Di Lin

Shenghai Yuan

Shengheng Liu

Shenghong He

Shenghong Yang

Shengjia Wang

Shengjun Zhou

Shengkai Zhang

Shengkun Xie

Shenglin Ma

Shengnian Wang

Shengqiang Wang

Sheng-Sheng Yu

Sheng-Shih Wang

Shengxiang Ge

Shengyang Chen

Shengyu Duan

Sheraz Aslam

Sherief Hashima

Sheroz Khan

Shervin Hashemi

Shervin Minaee

Shervin R. Arashloo

Sheryl Brahnam

Shiba Kuanar

Shichang Xuan

Shichao Ding

Shichao Jin

Shichao Kan

Shichao Niu

Shidan Wang

Shideh Kabiri Ameri

Shieh-Kung Huang

Shien Ri

Shien-Fong Lin

Shigang Li 
Shigeru Toyama

Shih-Chieh Chen

Shih-Chun Lin

Shih-Hau Fang

Shih-Jeh Wu

Shih-Kun Liu

Shihong Xia

Shi-Huang Chen

Shih-Wei Sun

Shih-Wei Tan

Shih-Yu Li

Shijie Feng

Shijing Sun

Shi-Jinn Horng

Shijun Liu

Shikuan Yang

Shilong Sun

Shimaa A. Abdel Hakeem Abdelhakeem

Shiming He

Shimshon Belkin

Shin Hur

Shin Jun Park

Shinae Ahn

Shinfeng Lin

Shinichi Sato

Shinichi Sobue

Shinichi Yamagiwa

Shinichiro Okazaki

Shinichiro Omachi

Shinji Kajimoto

Shinkyu Shinkyu Park

Shinnazar Seytnazarov

Shinpei Ogawa

Shintaro Izumi

Shintaro Sukegawa

Shinya Ikeno

Shiqiao Qin

Shirin Malihi

Shirsendu Sikdar

Shisheng Lin

Shishir Priyadarshi

Shisong Cao

Shitala Prasad

Shitao Zhu

Shiuh-Chuan Her

Shiv K. Sharma

Shivaram Arunachalam

Shivesh Kumar

Shi-Wei Lo

Shiwei Mo

Shiwen Mao
Shixi Yang

Shiyang Li

Shiyang Tang

Shiyi Tian

Shiyu Xie

Shi-yuan Han

Shiyuan Wang

Shizhi Chen

Shlomo Greenberg

Shmuel Springer

Shoaib Azam

Shogo Okamoto

Shohei Naito

Shoji Kakio

Shotaro Yoshida

Shou-Hu Xuan

Shoujun Xu

Shoukun Wang

Shoushui Wei

Show-Shiow Tzeng

Shriram S. Rangarajan

Shu Gong

Shu Lei

Shu Ting Goh

Shuai Fan

Shuai Gao

Shuai Li

Shuai Liu

Shuai Wang

Shuai Zhang

Shuai Zhao

Shuaiqi Liu

Shuaiyong Li

Shuanfeng Zhao

Shuang Du

Shuang Li

Shuang Liu

Shuang Mei

Shuang Song

Shuang Zhang

Shuang-Jian Jiao

Shuangquan Wang

Shuangshuang Fan

Shuangyi Wang

Shuangzhuang Guo

Shu-Chuan Chu

Shu-Cing Wang

Shufeng Li

Shuhua Fang

Shuicai Wu

Shuihua Jiang 
Shuiliang Fang

Shuilong Shen

Shuisen Chen

Shuja Ansari

Shu-Kai S. Fan

Shukui Liu

Shuming Jiao

Shu-Ming Tseng

Shuming Yang

Shumit Saha

Shun $\mathrm{Hu}$

Shun Wang

Shunbo Li

Shun-Hsi Hsu

Shun-Hsyung Chang

Shunjun Wei

Shunki Itadera

Shunmei Meng

Shunping Zhang

Shun-qing Ren

Shunsuke Nagahama

Shunsuke Shigaki

Shunsuke Tomita

Shunsuke Yoshimoto

Shu-Nung Yao

Shunyun Chen

Shuo Gao

Shuo Li

Shuo Shi

Shuo Wang

Shuo Yang

Shuohong Wang

Shuo-Tsung Chen

Shupeng Liu

Shuping $\mathrm{Wu}$

Shurong Dong

Shurui Fan

Shusheng Bi

Shushi Namba

Shuting Chen

Shuting Yang

Shuxiao Li

Shu-Yi Tu

Shuyin Xia

Shuyuan Xiao

Shuzeng Zhang

Shu-Zhi Liu

Shyan-Ming Yuan

Shyi-Chy Cheng

Sibel Cetinel

Siddharth Saksena
Siddhartha Khare

Siddheswar Maikap

Sidney Bruce Shiki

Sidney Gauthreaux

Sidorov Nikolay V.

Sierra Young

Sifat Muin

Sigal Portnoy

Sigitas Tamulevičius

Sigurd Eskeland

Sihyung Lee

Sikai Chen

Sikha Bagui

Sikora Wojciech

Siliang Lu

Silke Anna Theresa Weber

Silvana Gómez-Meire

Silvano Donati

Silvano Dragonieri

Silvano Valandro

Silvestro Roatta

Silvia Arazuri

Silvia Biasotti

Silvia Ceccacci

Silvia Krug

Silvia Liberata Ullo

Silvia Martínez Satorres

Silvia Mastrolembo Ventura

Silvia Miguel

Silvia Mirri

Silvia Mura

Silvia Ortín

Silvia Picciolini

Silvia San Román Mata

Silvia Sangiovanni

Silvia Ullo

Silvie Bernatová

Silvina Fais

Silvio Assis De Oliveira-Junior

Silvio Cocuzza

Silvio Lorenzetti

Silvio Nocera

Silvio Sabatini

Silvio Sampaio

Silvio Sciortino

Silvio Simani

Silviu Butnariu

Silviu Nastac

Sima Noghanian

Simas Rackauskas

Simeone Dussoni 
Simon Annaheim

Simon Avrillon

Simon Gay

Simon $\mathrm{Hu}$

Simon J. Bleiker

Simon Krašna

Simon Pezzutto

Simon Schweidler

Simon Taylor

Simon Thibault

Simon Tomažič

Simona Bianco

Simona Di Meo

Simona Kubíková

Simona Miclaus

Simona Moldovanu

Simona Petrakieva

Simona Ramanauskaitè

Simona Riurean

Simona Tecco

Simona Viorica Halunga

Simonas Ramanavicius

Simone Arena

Simone Baldi

Simone Battistini

Simone Borri

Simone Cosoli

Simone Dal Zilio

Simone Di Domenico

Simone Ferrari

Simone Fiori

Simone Genovesi

Simone Lolli

Simone Mantellini

Simone Mentasti

Simone Morosi

Simone Santoro

Simone Schuerle

Simone Sternini

Simone Zanotto

Simonetta Capone

Sina David

Sinan Sousan

Sin-Chi Kuok

Sitakanta Satapathy

Siti Hajar Othman

Siting Liu

Siting Xiong

Siu Shing Man

Siya Huang

Siyan Ma
Skanda Vivek

Skibicki Jacek

Sky Lee

Slaven Abdović

Slavenko Stojadinovic

Slavica Malinovic-Milicevic

Slavisa Tomic

Slavko Rupcic

Slavomír Labant

Slawomir Blonski

Sławomir Cięszczyk

Slawomir Gonkowski

Sławomir Gryś

Sławomir Hausman

Slawomir Judek

Slawomir Klos

Sławomir Kocoń

Slawomir Sujecki

Sławomir Wierzbicki

Slim Naifar

Slimane Larabi

Slobodan Babic

Slobodan Radojevic

Smolovik Mikhail

Smriti Bhatt

Snježana Firšt Rogale

Sobhan Roshani

Sobhan Soleymani

Socrates Basbas

Sofi Arshavsky-Graham

Sofia Balula Dias

Sofia Scataglini

Sofiane Boucenna

Soha Ahmadi

Soheil Keshmiri

Soheil Sadeghi Eshkevari

Sohel Rana

Sohrab Ferdowsi

Soi Hoi Lam

Sokratis Tsantis

Soldado Cabezuelo Ana Belén

Solmaz S. Kia

Somasekhar R. Chinnadayyala

Somayeh B. Shafiei

Somayyeh Asgari

Somdip Dey

Someshwar Roy

Son Thanh Nguyen

Son Trinh-Van

Song Feng

Song Joo Lee 
Song Li

Song Zhou

Songhao Shang

Songlin Zhang

Songling Huang

Songrui Wei

Songtao Xue

Sonia Gouveia

Sonia Grego

Sonia Helena Contreras-Ortiz

Sonia Ortiz-Peregrina

Sónia Simões

Sonja Gamsjaeger

Sonja Georgievska

Soo Young Shin

Soochang Park

Sook-Ling Chua

Sook-Yee Chong

Sooman Lim

Soon Hock Ng

Soonjae Ahn

Soon-Jae Kweon

Soonmin Seo

Soonseok Song

Soonwook Kwon

Soon-Yong Park

Soo-Whan Chung

Soo-Whang Baek

SooYoung Kwak

Sophia Diana Rozario

Sorana Bolboaca

Sorin Barabas

Sorin Ciortan

Sorin Hintea

Sorin Vlase

Sorinel Căpușneanu

Sossena Wood

Sotirios Diamantas

Sotirios Kontogiannis

Sotiris Avgousti

Sotiris E. Nikoletseas

Sotiris Kotsiantis

Soumya Manna

Soundaram Jeevara

Sourabh Solanki

Sourangsu Banerji

Sourav Rej

Sourav Sen Gupta

Souvik Ghosh

Sowon Hahn

Soyeon Lee
So-Yeon Yoon

Spandan Roy

Spartacus Gomariz

Spiridoula V. Margariti

Spiros A. Kostopoulos

Spiros Bezantakos

Spiros D. Dafnis

Spiros Georgakopoulos

Spyridon Blionas

Spyridon K. Chronopoulos

Spyridon Kintzios

Spyridon Vosinakis

Spyros Hirdaris

Srećko Krile

Sreejesh Sreedharan

Sreekar Babu Marpu

Srete Nikolovski

Sridhar Arjunan

Srinivas Hanasoge

Srinivas Kota

Sri-Rajaesekhar Kothapalli

Stamatios Amanatiadis

Stamatis Karlos

Stan Marius

Stanislao Grazioso

Stanislav A. Evlashin

Stanislav Harizanov

Stanislav Kurajica

Stanislav Popelka

Stanislav Trdan

Stanislav Zvánovec

Stanislavas Dadelo

Stanisław Bacior

Stanisław Deniziak

Stanisław Duer

Stanislaw Flaga

Stanisław Hożyń

Stanisław Saganowski

Stanisław Stopiński

Stanisław Szombara

Stanley Udochukwu Ofoegbu

Stavros Dimopoulos

Stavros Karkanis

Stavros Kolios

Stavros Koulouridis

Stavros Lazarou

Stavros Souravlas

Stavros Vologiannidis

Stavroula Kordella

Stavroula Ntoa

Stavroulakis Georgios 
Stef Vandermeeren

Ştefan Bodi

Stefan Domek

Stefan Fischer

Stefan Forstner

Stefan Gheorghe Pentiuc

Stefan Gumhold

Stefan K. Ehrlich

Stefan Knauth

Stefan Kolev

Stefan Küchemann

Stefan Lüdtke

Stefan Lüth

Stefan Mihaicuta

Stefan Naydenov

Stefan Panic

Stefan Pratschner

Stefan Sauermann

Stefan Schneider

Stefan Tabacu

Stefan Thomas

Stefan Wallner

Stefan Weber

Stefan Zauscher

Stefania Costantini

Stefania Federici

Stefania Marsili

Stefania Montani

Stefania Romeo

Stefania Tamburrini

Stefania Viti

Stefano Amatori

Stefano Bettati

Stefano Brusaporci

Stefano Caputo

Stefano Carrino

Stefano Del Sordo

Stefano Domek

Stefano Elia

Stefano Fornasaro

Stefano Forti

Stéfano Frizzo Stefenon

Stefano Guarino

Stefano Lai

Stefano Laureti

Stefano Lenci

Stefano Mangione

Stefano Mariani

Stefano Mauro

Stefano Palermi

Stefano Parrino
Stefano Pastorelli

Stefano Perilli

Stefano Pinardi

Stefano Primatesta

Stefano Quer

Stefano Rinaldi

Stefano Rossi

Stefano Salata

Stefano Salvatori

Stefano Sapienza

Stefano Selleri

Stefano Seriani

Stefano Sfarra

Stefano Stassi

Stefano Tennina

Stefano Tomasin

Stefano Zampolli

Stefano Zucca

Steffen Finck

Steffen Wendzel

Steffen Zeuch

Štefica Mrvelj

Stefka Atanassova

Stela-Maria Pruneanu

Stelian Alexandru Borz

Stelian Lupu

Stelios Ioannou

Stelios K. Georgantzinos

Stelios Zimeras

Stella Hrehová

Sten Hanke

Stepan Papacek

Stephan Jonas

Stephan Kallweit

Stephan M. Jonas

Stephan Warnat

Stephane Barland

Stephane Guinard

Stéphane Perrey

Stephane Rioual

Stéphane Roux

Stephanie R. Rogers

Stephanie Valentin

Stephen Bruder

Stephen Cain

Stephen Canfield

Stephen Czarnuch

Stephen G. Wilson

Stephen Mihailov

Stephen Monk

Stephen Morgan 
Stephen Powers

Stephen Schultz

Stephen U. Egarievwe

Stephen Weis

Steve Beeby

Steve Chan

Steve Glass

Steve Ling

Steve Mann

Steve O'Hern

Steve Rothberg

Steve Semancik

Steven Chatterton

Steven Hobbs

Steven J. Johnston

Steven J. Steinberg

Steven Latré

Steven Le Cam

Steven Levy

Steven M. Peterson

Steven P. Bennett

Steven Ross Murray

Steven Van Winden

Stoyan Slavov

Strahinja Dosen

Stuart McErlain-Naylor

Stuart Perry

Styliani Verykokou

Stylianos D. Assimonis

Stylianos Mystakidis

Stylianos Siskos

Su Man Nam

Su Zhang

Suan Lee

Suat Mercan

Subhamoy Mandal

Subhash Singh

Subhashis Hazarika

Subhasis Thakur

Subhayan Mukherjee

Subhransu Sahoo

Subodh Dhakal

Subrata Chakraborty

Suchinder K. Sharma

Sudarsana Reddy Kadiri

Sudheer Kumar Battula

Sudipta Banerjee

Suhas Somnath

Suhua Tang

Suh-Yeon Dong

Suhyun Park
Suixin Liu

Sujat Sen

Sujita Narayan

Sujoy Ghosh

Sujoy Ghosh Hajra

Suk Jin Lee

Sukanta Kumer Shill

Sukho Lee

Suku Nair

Sulaymon L. Eshkabilov

Süleyman Fatih Özmen

Sultan Mahmud

Sultan Shoaib

Sumana Biswas

Sumin Kim

Sun K. Hong

Sun Kwon Kim

Sun Woog Kim

Sunan Huang

Suncheol Kwon

Sunday Ekpo

Sung Min Park

Sung Yang

Sunggu Lee

Sunghae Jun

Sung-Hak Lee

Sung-Ho Bae

Sungho Jeon

Sungho Kim

Sungho Lee

Sunghyup Sean Hyun

Sungjun Kim

Sungkyu Shaun Park

Sung-Liang Chen

Sung-Man Kim

Sung-Min Hong

Sung-Phil Kim

Sungtae Shin

Sungtek Kahng

Sung-won Cho

Sungwook Cho

Sungwook Mhin

Sungyong Choi

Sung-Yong Son

Sung-Yun Park

Sunil Chakrapani

Sun-Joo Jang

Sunmean Kim

Sunny Raj

Sunwook Kim

Sunwoong Choi 
Suoda Chu

Suparerk Janjarasjitt

Supriyo Bandyopadhyay

Supriyo Ray

Suraparb Keawsawasvong

Suren Kumar

Surender Reddy Salkuti

Suresh Neethirajan

Suryadipta Majumdar

Susan Ann O'Shaughnessy

Susan M. Wernimont

Susan Zhou

Susana Catarino

Susana Dias Amaral

Susana Fernández

Susana Hornillo Mellado

Susana Palma

Susana Silva

Susanna Kaiser

Susanna Spinsante

Susanna Summa

Susanne M. Van Der Veen

Sushanta Mitra

Sushil Kumar Singh

Sushruta Surappa

Susil Baral

Susmit Shannigrahi

Susumu Ishihara

Susumu Okumura

Sutanu Bhowmick

Sutripto Majumder

Suyun Ham

Suzana Uran

Suzanne E. Blatt

Suzy Ngomo

Svatopluk Matula

Sven G. Bilén

Sven Mayer

Svetla Stoilova

Svetlana Boudko

Svetlana Girs

Svetlana Khonina

Svetlana Markova

Svetlana N. Khonina

Svetlana Von Gratowski

Svilen Petrov Sabchevski

Svitlana Kopyl

Swaminath Venkateswaran

Swati J. Patil

Syed A. Bukhari

Syed Afa Ali Shah
Syed Agha Hassnain Mohsan

Syed Ali Kalangala Sikkanther Batcha

Syed Aziz Shah

Syed Basit Ali Bukhari

Syed Furqan Qadri

Syed Khairul Bashar

Syed Muhammad Anwar

Syed Muhammad Asad

Syed Muhammad Usama

Syed Muzahir Abbas

Syed Tahir Hussain Rizvi

Syed Tariq Shah

Syed Thouheed Ahmed

Syed Turab Raza

Syeda Adila Afghan

Syeda Fizzah Jilani

Syed-Abdul Shabbir

Sylvain Bertrand

Sylvain Feruglio

Sylvain Girard

Sylvain Grenier

Sylvain Lecler

Sylvain Magne

Sylvain Mezil

Sylvie Chambon

Sylvio Barbon Junior

Sylwester Borowski

Sylwia Baluta

Sylwia Szczęśniak

Sylwia Szporak-Wasilewska

Sylwia Werbińska-Wojciechowska

Synh Viet-Uyen Ha

Sytze De Bruin

Syuichi Itahashi

Szczepan Paszkiel

Szilard Csizmadia

Szilvia Nagy

Szücs Veronika

Szymon Chorazy

Szymon Cyfert

Szymon Łoś

Szymon Malinowski

Szymon Ogonowski

Szymon Sieciński

Szymon Wójtewicz

Tad Brunye

Tad Gonsalves

Tadamasa Sawada

Tadashi Ito

Tadeusz Głowacki

Tadeusz Mikolajczyk 
Tadeusz Pisarkiewicz

Tadeusz Sondej

Tadeusz Stepinski

Tae Joon Jun

Tae Keun Yoo

Tae-Eung Sung

Taeho Jung

Taehoon Kim

Taejoon Kim

Tahir Khurshaid

Tahmid Latif

Tahseen A. Alwattar

Tai Fei

Taihong Liu

Taimur Hassan

Taiping Zeng

Tairan Liu

Taisong Pan

Taiwen Tang

Taiyong Li

Takafumi Ando

Takahiro Arakawa

Takahiro Kanno

Takahiro Kuchimaru

Takahiro Kunimine

Takamichi Miyata

Takashi Chiba

Takashi Iida

Takashi Isezaki

Takashi Okayasu

Takashiro Akitsu

Takehisa Hanawa

Takeshi Fujino

Takeshi Go Tsuru

Takeshi Hashishin

Takeshi Kamiya

Takeshi Yanagida

Takeyasu Sakai

Takuma Akimoto

Takumi Asakura

Takumi Okabe

Takuya Kiyokawa

Tales Cleber Pimenta

Talles Marcelo G De A Barbosa

Tam Nguyen

Tamara Bonaci

Tamara Radivilova

Tamás Bécsi

Tamas Haidegger

Tamas Orosz

Tamás Pardy
Tamás Ruppert

Tamas Varga

Tamio Ikehashi

Tan Jen Hong

Tan Yigitcanlar

Tanesh Kumar

Tania García

Taniguchi Naokazu

Tanja Dudenbostel

Tanju Yildirim

Tanmay Kulkarni

Tanveer Ahmad

Tanweer Alam

Tanweer Ali

Tanya Hutter

Tanzilur Rahman

Tao Cao

Tao Chen

Tao Hong

$\mathrm{Tao} \mathrm{Hu}$

Tao Huang

Tao Jiang

Tao Lei

Tao Li

Tao Liu

Tao Luo

Tao Shaohua

Tao Shen

Tao Wang

Tao Wu

Tao Yang

Tao Yin

Tao Yue

Tao Zhang

Tao Zhou

Tao-Hsing Chen

Tara Julia Hamilton

Țarălungă Dragoș Daniel

Tarek Berghout

Tarek Djerafi

Tarek Elfouly

Tarek Gaber

Tarek Youssef

Tareq Assaf

Tareq Khan

Tarig Ali

Tariq Abdullah

Tariq Ali

Taro Ueda

Taron Makaryan

Tasadduq Imam 
Tasneem Darwish

Tatevik Chalyan

Tatiana Atanasova

Tatiana Jaworska

Tatiana Kováčiková

Tatiana Kuchmenko

Tatiana Latychevskaia

Tatiana M. Pinho

Tatjana Gric

Tatsiana Mikulchyk

Tatsuaki Tagami

Tatsuo Yoshinobu

Tatsuro Goda

Tatsuya Iwata

Tatu Kumpulainen

Tauhidul Alam

Tauseef Jamal

Tawfik Khattab

Tawfik Najeh

Tawhidul Islam Khan

Taylor Chomiak

Te Han

Tea Duplančić Leder

Tecla Bonci

Teddy Craciunescu

Tee Hui Teo

Teemu Räsänen

Teen-Hang Meen

Teerawat Kumrai

Tejasvi Parupudi

Te-Jen Su

Telmo Fernandes

Temple Grandin

Teng Li

Tengfei Chang

Tengfei Long

Teodor B. Iliev

Teodor Toth

Teodorico Caporaso

Teodoro Georgiadis

Teodoro Montanaro

Teresa Albero-Albero

Teresa Horton

Teresa Nogueira

Teresa Santos

Teresa Zawadzka

Teresa Zwierko

Terézia Pošiváková

Terje Tjelta

Terrence J. Moore

Tessai Hayama
Tetiana Manyk

Tetsuo Iguchi

Tetsuo Sasano

Tetsuro Yanaseko

Tetsushi Ikeda

Tetsuya Kawanishi

Thabet Kacem

Thad Walker

Thaís De Sá

Thanasis Koutras

Thanasis Loukopoulos

Thanh Hai Le

Thanh Luan Phan

Thanh Nguyen

Thanh Nho Do

Thanh-Canh Huynh

Thanikanti Sudhakar Babu

Thar Baker

Tharmalingam Sivarupan

Thejas Gubbi Sadashiva

Theo G. Kanter

Theodor D. Popescu

Theodore A. Corcovilos

Theodore Kotsilieris

Theodore Zahariadis

Theodoros Karakasidis

Theodosios Sapounidis

Theofilos Chrysikos

Theofilos Papadopoulos

Theoklis Nikolaidis

Thiago M. B. F. Oliveira

Thiago Poleto

Thiago Sequinel

Thibault Laplace

Thibaut Raharijaona

Thien D. Nguyen

Thien Huynh-The

Thierry Baccino

Thierry Oscar Edoh

Thilo Sandner

Thomas Bretterklieber

Thomas Charrett

Thomas Cilloni

Thomas Gittler

Thomas Heide Clausen

Thomas Heinze

Thomas Howard

Thomas J. Smith

Thomas Kletschkowski

Thomas Konrad

Thomas Lagkas 
Thomas Lango

Thomas M. Arruda

Thomas M. Deserno

Thomas M. Kaiser

Thomas M. Kraft

Thomas Ott

Thomas Purfürst

Thomas Scott

Thomas Sheppard

Thomas Steffen

Thomas Stewart

Thomas Wiemann

Thomas Zefferer

Thran Van Thuc

Thyago Nepomuceno

Tiago Almeida Silva

Tiago Bresolin

Tiago Fernandes Tavares

Tiago Nascimento

Tiago Varum

Tian Jin

Tian Li

Tian Qiu

Tian Tian

Tian Xin

Tian Zhou

Tiancheng Li

Tianfeng Gu

Tian-Fu Lee

Tianhong Dai

Tianhua Xu

Tianlin Wang

Tianqi Yu

Tianshu Qu

Tianxing Li

Tianxun Gong

Tianyiyi He

Tiberiu Stefan Letia

Tiberiu-Paul Neagu

Tibor Guzsvinecz

Tibor Hianik

Tibor Krenicky

Tibor Pasinszki

Tibor Szkaliczki

Tie Zhang

Tiebiao Zhao

Tiejiong Lou

Tielin Shi

Tieling Zhang

Tien-Dung Nguyen

Tien-Lung Sun
Tien-Thong Nguyen Do

Tien-Yin Chou

Tie-Qiang Li

Tieshan Li

Till Nierhaus

Tim A. Majchrzak

Tim Claudius Stratmann

Tim Molteno

Tim Morris

Tim Verbelen

Tim W. C. Brown

Timea Ignat

Timofey Shevgunov

Timothy M. Young

Timothy Otim

Timothy Patten

Timothy S. De Smet

Timothy Sands

Timothy Vincent

Tina Bobić

Tina McKay

Tina Tičinović Kurir

Tina Vrabec

Ting Lei

Ting Li

Ting Yun

Ting Zou

Ting-Chia Ou

Tingguang Ma

Tingna Shi

Tingrui Liu

Tingting Lang

Tingting Sui

Tingting Yang

Titan Paul

Titus Sandu

Tiziana C. Bond

Tiziana Segreto

Tiziano Zarra

Tjin Chuan

Toan Dinh

Toan Duc Cao

Tobias Günther

Tobias Habisreuther

Tobias Kalisch

Tobias Meisen

Tobias Nilsson

Tobias Schripp

Tobias Storch

Toby J. Ellmers

Todd Moon 
Todd Richards

Todor Cooklev

Todor Ganchev

Todor Tagarev

Tohru Sasaki

Tom Foulsham

Tom Lahmer

Tom van der Sande

Tomas Baležentis

Tomás Gómez Álvarez-Arenas

Tomás González

Tomas Horvath

Tomas Janda

Tomas Sochor

Tomáš Zálabský

Tomas Zikmund

Tomasz Bakon

Tomasz Bieniek

Tomasz Blicharski

Tomasz Cholewa

Tomasz Cieplak

Tomasz Cudejko

Tomasz Dysarz

Tomasz Górecki

Tomasz Grzes

Tomasz Hachaj

Tomasz Halski

Tomasz Kapuscinski

Tomasz Kik

Tomasz Kisielewicz

Tomasz Klekiel

Tomasz Kloda

Tomasz Kryjak

Tomasz Kulczyk

Tomasz Lipecki

Tomasz Marciniak

Tomasz Mościcki

Tomasz Niedoba

Tomasz Norbert Kołtunowicz

Tomasz Orczyk

Tomasz Osuch

Tomasz Oszako

Tomasz Pander

Tomasz Rogalski

Tomasz Rokicki

Tomasz Rymarczyk

Tomasz Sipko

Tomasz Starecki

Tomasz Stoch

Tomasz Szczegielniak

Tomasz Szymborski
Tomasz Teleszewski

Tomasz Templin

Tomasz Tokarek

Tomasz Wandowski

Tomasz Wasilewski

Tomasz Wegiel

Tomasz Winiarski

Tomasz Wolny

Tomaž Ambrožič

Tomaz Javornik

Tomaz Kosar

Tomaž Podobnikar

Tomislav Bašić

Tomislav Ivanjko

Tomislav Keser

Tomislav Kos

Tomislav Matic

Tomislav Medic

Tomislav Petković

Tommaso Bacci

Tommaso Ercoli

Tommaso Lomonaco

Tommaso Pecorella

Tomoaki Kunugi

Tomohiro Hayashi

Tomohiro Mashita

Tomohisa Seki

Tomohito Sekine

Tomokazu Hattori

Tomotsumi Fujisawa

Tomoya Takada

Tonći Modrić

Tongwen Li

Tong-Xing Zheng

Tonino Traini

Tony Lindeberg

Tony Luczak

Tony Waterhouse

Tony Zhang

Toomas Rang

Toomas Vaimann

Torbjørn Skramstad

Toru Kurihara

Toru Wada

Toshiaki Hattori

Toshiaki Omori

Toshiaki Tsuji

Toshihiko Yoshino

Toshihiro Shimada

Toshikazu Shinba

Toshiki Kanamoto 
Toshiya Arakawa

Touqeer Ahmad

Tracy Anne Hammond

Traianos V. Yioultsis

Trent Biggs

Trevor Benson

Tri Dev Acharya

Tribikram Kundu

Triet Nguyen-Van

Trifol Jon

Trung Thanh Ngo

Truong Xuan Do

Tsang-Long Pao

Tsan-Pin Wang

Tsimpiris Alkiviadis

Tsubasa Maruyama

Tsubasa Minematsu

Tsukuru Minamiki

Tsuneo Horiguchi

Tsung-Han Tsai

Tsung-Hung Lin

Tsung-Lin Chen

Tsung-Rong Kuo

Tsung-Yu Chou

Tsutomu Tsuboi

Tu Le Manh

Tuan Anh Nguyen

Tuan Dang

Tuan-Minh Pham

Tuan-Tu Huynh

Tuanwei $\mathrm{Xu}$

Tuba Yilmaz

Tudor Deaconescu

Tudor Ionescu

Tudor Palade

Tugba Kilic

Tuğrul Oktay

Tu-Liang Lin

Tung-Ching Su

Tung-Hsun Hsieh

Tural Khudiyev

Turker Dagdelen

Tushar Kanti Roy

Tutomu Murase

Tyler N. Tallman

Tymoteusz Horbiński

Tzirakis Panagiotis

Tzu-Chieh Chou

Tzu-En Lin

Tzu-Hsien Sang

Tzung-Shi Chen
Uchenna Daniel Ani

Udo Frese

Udo Netzelmann

Ugur Alganci

Uikyum Kim

Ulf Jensen

Ulises Orozco

Ulises Páramo-García

Ulises Pineda Rico

Ullah Amin

Ulrich Balss

Ulrich Guth

Ulrich Schreiber

Ulrike Klueh

Ulrike Lechner

Uma Shahani

Umar Iqbal

Umar Zakir Abdul Hamid

Umberto Amato

Umberto Andriolo

Umberto Papa

Umberto Riccardi

Umer Javed

Umer Mehmood

Umit Ogras

Un Ji

Unal Devrim

Unal Zak Sakoglu

Upul Gunawardana

Uroš Klanšek

Urs U. Graf

Urszula Marmol

Urtè Bubnienè

Usman Habib

Usman Rashid

Usman Saeed Tariq

Ute Rabe

Utkarsh Kohli

Uwe Brand

Uwe Hampel

Uwe Pliquett

Uwe Serdült

Uznir Ujang

Vacius Jusas

Vaclav Bunc

Vaclav Hlavac

Vaclav Krivanek

Vaclav Kubecek

Václav Navrátil

Vaclav Prajzler

Václav Ranc 
Vaclava Piorecka

Vadim A. Parfenov

Vadim Agafonov

Vadim Grubov

Vadim Parfenov

Vadim S. Tynchenko

Vadim Zhmud

Vageeswar Rajaram

Vahab Youssof Zadeh

Vahid Abolghasemi

Vahid Yaghoubi

Vaibhav Shah

Vaidas Lukoševičius

Vaidotas Marozas

Válber César Cavalcanti Roza

Valderi Reis Quietinho Leithardt

Valdson J. Silva

Valentín Barral

Valentin Bégel

Valentin Buiculescu

Valentin Cardeñoso-Payo

Valentin Gomez-Jauregui

Valentin Mateev

Valentín Osuna-Enciso

Valentina Alena Girelli

Valentina Bucciarelli

Valentina De Simone

Valentina Franzoni

Valentina Grippo

Valentina Longo

Valentina Yakovleva

Valentino Meacci

Valentyn Korobiichuk

Valeri I. Kovalev

Valeria Belluscio

Valeria Loscri

Valeria Rodionova

Valeria Rosso

Valeria Spizzichino

Valeria-Ersilia Oniga

Valério Antonio Pamplona Salomon

Valerio Biancalana

Valerio Ferroni

Valerio Scordamaglia

Valeriu Manuel Ionescu

Valeriy Krivetskiy

Valery Tsaplev

Valéry U. Zavorotny

Valery V. Kalinchuk

Valner Brusamarello

Van Dung Nguyen
Van-An Duong

Van-Doan Nguyen

Vanessa Camilleri

Vangelis Oikonomou

Vangelis Sakkalis

Vanlin Sathya

Van-Thai Tran

Varadraj Prabhu Gurupur

Varghese Kurian

Varnakavi Naresh

Varun Lingaiah Kopparthy

Varun Vyas

Vasco Pereira

Vasco Soares

Vasile A. Popescu

Vasile Chis

Vasile Daniel Pavaloaia

Vasile Gheorghita Gaitan

Vasile Marinca

Vasile Palade

Vasile Paul Bresfelean

Vasile Preda

Vasile Sima

Vasile-Gabriel Iana

Vasileios Kalogirou

Vasileios Karyotis

Vasileios Komianos

Vasileios Vlachos

Vasiliki Vita

Vasilios Liordos

Vasilis Christofilakis

Vasilis K. Dertimanis

Vasilis Kanakoudis

Vasiliy Elagin

Vasiliy Osipov

Vasko Jovanovski

Vassiliki Kontargyri

Vassilios C. Moussas

Vassilis C. Moulianitis

Vassilis G. Kaburlasos

Vassilis George Aschonitis

Vassilis Moulianitis

Vassilis S. Kodogiannis

Vedhus Hoskere

Vedran Mrzljak

Vedrana Jerkovic Stil

Vedyagin Aleksey

Velislava Lyubenova

Venkata Krishna Karthik Tangirala

Venkata Manthina

Venkata Prasanth Yanambaka 
Venkata Prashant Modekurthy

Venkata Puli

Venkatakrishnan Rengarajan

Venkateswaran Narayanaswamy

Venkateswaran Vivekananthan

Vera Gramigna

Veraldo Liesenberg

Veronica Maier

Verónica Montes García

Veronica Pazzi

Veronica Perez-Cabezas

Veronica Rossano

Veronica Sberveglieri

Verónica Serafín

Veronika Szucs

Verrier Isabelle

Veselin N. Ivanovic

Veselin Rakocevic

Veselina Bureva

Vesna Babić

Vessela Krasteva

Vicente Duran-Bosch

Vicente Faus-Matoses

Vicente Feliu

Vicente González Ruiz

Vicente J.P. Amorim

Vicente Lucena

Vicente Romo Pérez

Victor Bloch

Victor Bocos-Bintintan

Victor Bolbot

Victor Chong

Víctor Corcoba Magaña

Victor Farm-Guoo Tseng

Víctor Gómez-Mayordomo

Victor Guerra

Victor H. Benitez

Victor Hugo Rohden Prudente

Victor Ilisie

Victor Joos

Victor Kallen

Victor Manuel González Suárez

Víctor Manuel Rivas Santos

Victor Petrov

Victor Ramos

Víctor Ruiz-Valdepeñas Montiel

Victor Sanchez

Victor Vlădăreanu

Victor Zhuravlev

Victoria Hodge

Victoria Ramos González
Vidal Moreno

Vignesh Shanbhag

Vijay Anand

Vijay Kakani

Vijay Kumar Sharma

Vijayakumar Anand

Vijaykumar B. Varma

Vijaykumar Rajasekaran

Vikas Kumar

Vikas Sharma

Vikrant Bhateja

Viksit Kumar

Viktor Medvedev

Viktor Skrickij

Viktor Vabson

Vilfredo De Pascalis

Ville Kaajakari

Ville Pulkki

Vilma Ratautaite

Vinaya Basavarajappa

Vinayakumar Ravi

Vincent E. Lamberti

Vincent Pey Chung

Vincent Sieben

Vincenzo Bonaiuto

Vincenzo Dentamaro

Vincenzo Deufemia

Vincenzo Eramo

Vincenzo Gallelli

Vincenzo Grassia

Vincenzo Loschiavo

Vincenzo Mallardo

Vincenzo Manuel Marzullo

Vincenzo Mariano Mastronardi

Vincenzo Pasquale Giofrè

Vincenzo Quinzi

Vincenzo Russo

Vincenzo Serlenga

Vineet Vajpayee

Vinh Nguyen

Vinicius Kartnaller

Vinícius Maran

Vinicius Rofatto

Vinod Belwanshi

Vinod Kumar

Violeta Lazic

Violeta Poenaru

Violeta Simion

Viorel Minzu

Viorel Paleu

Viraj Muthugala 
Virgil Dobrota

Virgil Dumbrava

Virgil Optasanu

Virgil-Florin Duma

Virgínia Fernandes Mota

Visar Farhangi

Vishal Singh

Visnja Stepanic

Vitali F. Nesterenko

Vitalii A Pavlov

Vitaly Levashenko

Vito Errico

Vito Fernicola

Vito Imbrenda

Vito Monaco

Vitoantonio Bevilacqua

Vitor Caldeirinha

Vítor João Pereira Domingues Martinho

Vitor N. Coelho

Vítor Viegas

Vittoria Bruni

Vittoria Perrotti

Vittorio B. Lippi

Vittorio Di Federico

Vittorio Memmolo

Vittorio Merlo

Vittorio Rampa

Vittorio Solina

Vivek Kumar

Vivek T. Rathod

Vivi Anggraini

Vivian Loftness

Viviana Mulloni

Viviane Pilla

Vlad Laurentiu David

Vlad Popescu

Vladan Papic

Vladana Grabez

Vladeanu Calin

Vladimir A. Sreckovic

Vladimir Anfinogentov

Vladimir Bochenkov

Vladimír Bulej

Vladimir D. Kuptsov

Vladimir Heiskanen

Vladimir I. Zverev

Vladimir Klimentievich Kachanov

Vladimir Knyaz

Vladimir Kodkin

Vladimir Kolesov

Vladimir Krishtop
Vladimir Krivovichev

Vladimir Kukushkin

Vladimir Kuts

Vladimir Kutuzov

Vladimir Medved

Vladimir Mirsky

Vladimir Nikolaevich Kostin

Vladimir Nikolayevich Soloviev

Vladimir Pitschmann

Vladimir Popok

Vladimir Popov

Vladimir Poulkov

Vladimir Rajs

Vladimir Rogalewicz

Vladimir Sergeyev

Vladimir Shakhov

Vladimir Shepelev

Vladimir Skripnyak

Vladimir Sobeslav

Vladimir Soloviev

Vladimir Stankovic

Vladimir Sukhov

Vladimir Tadic

Vladimir Tadić

Vladimir V. Andreev

Vladimir Vavilov

Vladimir Villarreal

Vladimíra Biňasová

Vladislav Demyanov

Vladislav Kalinnikov

Vlastimil Masek

Vlastimil Matejec

Vojtěch Petrucha

Volker Cimalla

Volker Dworak

Volker Rodehorst

Volkov Petr

Volodymyr Gurski

Volodymyr Ponomaryov

Vrchota Jaroslav

Vuslat B. Juska

Vytautas Bučinskas

Waad Subber

Wacław Dziurzńsk

Wael Abdullah Ahmad

Wafa M. Batayneh

Wagner Coelho De Albuquerque Pereira

Wagner Tanaka Botelho

Wahyu Caesarendra

Wai Lok Woo

Wai-keung Fung 
Waldemar Dołęa

Waldemar Jendernalik

Waldemar Kociuba

Waldemar Odziemczyk

Waldemar Rączka

Waldemar Świderski

Walter Grondzik

Walter J. Salcedo

Wanda Forczek

Wanfeng Dou

Wang Jianjiang

Wang Tian

Wanguo Jiao

Wanjung Chang

Wanke Liu

Wankun Xie

Wanli Liu

Wann-Yun Shieh

Wansoo Nah

Wanxin Wen

Waqar Ahmad

Waqas Ahmed

Ward Van Der Tempel

Wassim Alexan

Wataru Sato

Wei Gao

Wei Guan

Wei Hu

Wei Jing

Wei Li

Wei Lin

Wei Liu

Wei Ma

Wei Ouyang

Wei Pu

Wei Qiu

Wei Shao

Wei Shen

Wei Song

Wei Tang

Wei Tao

Wei Wang

Wei Wu

Wei Yan

Wei Yang

Wei Yao

Wei Yi

Wei Zhang

Wei Zhao

Wei Zheng

Weibin Li
Weibing Gu

Weibing Zhong

Weibo Liu

Weichao Zhuang

Wei-Che Chien

Weicheng Cui

Wei-Chiang Hong

Wei-Chiang Wu

Weidang Lu

Weidong Yang

Weifeng Pan

Weiguang Zhang

Weiguo Fang

Wei-Han Chen

Weihe Xu

Wei-hsiung Yang

Wei-Hsun Lee

Weihua Dong

Wei-Hua Hu

Weihua Liu

Weihua Pei

Weijie Fu

Weijie Li

Weijie Liu

Wei-Jiun Su

Weili Deng

Weilin Luo

Weilin Ye

Wei-Lung Tseng

Weiping Li

Weiping Zhu

Weiqi Hua

Weiqiang Ma

Wei-Rong Liu

Weisheng Chiu

Wei-Ting Lin

Weiting Liu

Weiwei Liu

Weiwei Sun

Weiwen Zhang

Weixi Gu

Weiyan Hou

Weizhi Meng

Wellington Pinheiro Dos Santos

Wen Chang Huang

Wen Liu

Wen Sun

Wen Xiao

Wen Zhang

Wenbin Yu

Wen-Chang Tsai 
Wen-Chung Tsai

Wenfang Lu

Wengang $\mathrm{Wu}$

Wengang Zhang

Wen-Hsi Cheng

Wenjian Hua

Wenjin Wang

Wenjin $\mathrm{Wu}$

Wen-Jong Chen

Wenjuan Sun

Wenjun (Chris) Zhang

Wenkai Li

Wenlin Gong

Wenming Chen

Wenpeng Li

Wenqi Ren

Wenqiang Zhang

Wen-Sheng Zhao

Wensong Jiang

Wentao Li

Wentao Zhang

Wenxin Dai

Wenxin Tian

Wen-Ya Lee

Wenyi Wang

Wenyu Cai

Wenyuan Cui

Wenyuan Yang

Wenzeng Zhang

Wenzhuo Wu

Werner Haselmayr

Werner Lienhart

Werner Mäntele

Weslania Viviane Nascimento

Wesley Baker

Wesley T. Honeycutt

Wiktoria Wojnicz

Wilfried Gappmair

Wilfried Uhring

Wilhelm Robert Grosz

William C. Wilson

William D.G. Brittain

William Headley

William Hurst

William L. Raffe

William Romine

William Stewart

William Y. Y. Cheng

William Zimmerman

Wim Ectors

Winston Percybrooks
Wioletta Ochędzan-Siodłak

Wisit Cheungpasitporn

Witold Czajewski

Witold Kazimierski

Władysław Skarbek

Włodzimierz Kasprzak

Wojciech Dawidowski

Wojciech Fabianowski

Wojciech Jadwicienczak

Wojciech Kaczmarek

Wojciech Knap

Wojciech Sałabun

Wojciech Sas

Wojciech Skierucha

Wojciech Skrzeczanowski

Wojciech Stecz

Wojciech Sumelka

Wojciech Szeląg

Wojciech Tutak

Wojciech Witkowski

Wojciech Wolanśki

Wojciech Zabierowski

Wolfgang Bacsa

Wolfgang Caliebe

Wolfgang Fuhl

Wolfgang Hübner

Wolfgang Leister

Wolfgang Potthast

Wolfgang Schreiner

Wolfram Luther

Wolfram Volk

Won-Du Chang

Won-Jae Ryu

Wonjin $\mathrm{Na}$

Wonjun Kim

Wonseok Lee

Wonzoo Chung

Woo June Choi

Woo-Kyung Sun

Woonghee Lee

Woo-Suck Han

Wooyeol Choi

Woubishet Zewdu Taffese

Wouter Van Verre

Wu Deng

$\mathrm{Wu}$ Xiao

Wu-Cheng Chi

Wujun Si

Wuyi Ming

Wuyi Wan

Wycislik Lukasz 
Wynand JvdM Steyn

X P V Maldague

Xabiel García Pañeda

Xanthoula Eirini Pantazi

Xavier Boddaert

Xavier Fernando

Xavier Gasparutto

Xavier Vilanova

Xénia Latypova

Xenofon Strakosas

Xi Gu

$X_{i}$ Peng

Xi Qiao

Xi Shi

Xi Yang

Xia Min

Xiadong Wang

Xian Jian

Xian Liu

Xian Sun

Xian Tao

Xianbo Wang

Xianfeng Yuan

Xiang Chen

Xiang Cheng

Xiang Li

Xiang Wu

Xiang Xie

Xiang $\mathrm{Xu}$

Xiang Zhang

Xiangang Luo

Xiangbo Kong

Xiangcheng Chen

Xianghong Wang

Xiang-Jun Zou

Xiangping Chu

Xiang-Tian Kong

Xiangxin Li

Xiangxiong Kong

Xiangyang Ju

Xiangyang $\mathrm{Xu}$

Xiangyin Zhang

Xiangyu $\mathrm{Xu}$

Xianjian Jin

Xianju Li

Xianjun Hao

Xianta Jiang

Xianyi Zeng

Xianzheng Zong

Xianzhi Wang

Xianzhong Chen
Xiao Dong Zhou

Xiao Fu

Xiao Li

Xiao Liang

Xiao Liu

Xiao Wang

Xiao Wu

Xiao Yong

Xiao Zhou

Xiaobing Dai

Xiaobing Li

Xiaobo Rui

Xiaochao Dang

Xiaochen Zhang

Xiaochen Zheng

Xiaodan Xi

Xiaodong Lu

Xiaodong Yang

Xiaofei Deng

Xiaofei Li

Xiaofeng Gao

Xiaofeng Li

Xiaofeng $\mathrm{Lu}$

Xiaofeng Wang

Xiaofeng Yuan

Xiaogang Deng

Xiaoguang Dong

Xiaohong Sui

Xiaohong Zhou

Xiaohua Hao

Xiaohui Han

Xiaohui Yang

Xiao-hui Yuan

Xiaojing Mu

Xiaojun Tang

Xiaojun $\mathrm{Yu}$

Xiaokai Wang

Xiaolei Yang

Xiaoli Xi

Xiaoliang Guo

Xiaoliang Meng

Xiaoliang Zhu

Xiaolin Chang

Xiaolin Ma

Xiaoling Zhang

Xiaoman Lu

Xiaoming Gao

Xiaoming Liu

Xiaoming Wang

Xiaoming $\mathrm{Wu}$

Xiaoning Jiang 
Xiaopeng Li

Xiaoping Liao

Xiaoping Liu

Xiaoqiang Hua

Xiaoqiang Li

Xiaoqing Bai

Xiaoqing Gao

Xiaoqing Tian

Xiaorong Zhu

Xiaoshan Bai

Xiaosheng Zhang

Xiaoshuang Chen

Xiao-Song Zhu

Xiaotong Zhang

Xiaowei Liu

Xiaowen $\mathrm{Xu}$

Xiaoxing $\mathrm{He}$

Xiaoyang Rebecca Li

Xiaoyi Bao

Xiaoying Guo

Xiaoying Zhuang

Xiaoyong Chen

Xiaoyu Cheng

Xiaoyu Wan

Xiaoyuan Wang

Xiaoyuan Zhu

Xiaozhen Zhou

Xi-chuan Liu

Xignpo Ma

Ximeng Cheng

Ximeng Liu

Xin Cheng

Xin Du

Xin Gao

Xin Li

Xin Lin

Xin Liu

Xin Ning

Xin Shan

Xin Wang

Xin Zhang

Xin Zhong

Xin Zhuang

Xinbin Li

Xincong Yang

Xing $\mathrm{Fu}$

Xing Ma

Xingchen Ji

Xingchen $\mathrm{Ma}$

Xingchen Yang

Xinge Yu
Xingfu Zhang

Xinghua Li

Xinghua Yang

Xingjian Liu

Xingliang Huo

Xingling Shao

Xinglong Ju

Xing-Ming Guo

Xingming Liang

Xingquan Cai

Xingran Cui

Xingyu Gu

Xingyu Lin

Xinheng Wang

Xinhua Mao

Xinke Li

Xinli Du

Xinming Li

XinMing Zhang

Xinqiang Chen

Xinqin Liao

Xinqun Zhu

Xinrui Ding

Xinyu Gu

Xinyu Li

Xinyu Zhao

Xinyuan Wei

Xinyue Shen

Xinzhong Li

Xiong Yang

Xiongbo Duan

Xiping $\mathrm{He}$

Xiqian Jiang

Xiu Li

Xiuhong Li

Xiujuan Chai

Xiukun Wei

Xiuping Liu

Xiuwen Fu

Xiuyou Han

Xiyu Shi

Xoan Xosé Fernández Sánchez-Romate

Xosé Antón Vila Sobrino

Xosé Luís Deán Ben

Xose M. Lopez-Fernandez

Xu An Wang

Xu Bai

Xu Ding

Xu Qiao

Xu Tang

Xuan Sun 
Xuan Zhu

Xuchun Gui

Xudong Jia

Xudong Li

Xudong Zhang

Xudong Zou

Xue Han

Xuebin Ren

Xue-Bo Jin

Xuedong Zhang

Xuefeng $\mathrm{Hu}$

Xuefeng Zhang

Xuejian $\mathrm{Wu}$

Xuejin Li

Xuejing Wang

Xuejun Zhang

Xuemei Zhou

Xuemin Chen

Xuesong Yan

Xuewen Wang

Xueyi Shang

Xuezhi Wang

Xuezhi Wu

Xuguang Lan

Xukun Yin

Xun Dou

Xuyang Zhang

Xuyuan Tao

Xxiaorui Shao

Yadong Zhou

Yael Nemirovsky

Yafeng Yin

Yago Diez

Yahui Peng

Yahya Meziani

Yahya Rharbi

Yajun Liu

Yakoub Bazi

Yakushenko Alexey

Yalan Liu

Yan (Rockee) Zhang

Yan Hong

Yan Huang

Yan Li

Yan Liu

Yan Ma

Yan Naung Soe

Yan Shi

Yan Wang

Yan Zhang

Yanbin Wang
Yanbo Niu

Yanbo Pan

Yanbo Yang

Yanchao Sun

Yanchao Zhang

Yanfeng Jiang

Yanfeng Shen

Yang $\mathrm{Hu}$

Yang Jun Kang

Yang Kailu

Yang Kuang

Yang Li

Yang Lin

Yang Lou

Yang Lu

Yang Ran

Yang Shao

Yang Shen

Yang Shuang

Yang Song

Yang Wang

Yang $\mathrm{Xu}$

Yang Yang

Yang Yu

Yang Yue

Yang Zhao

Yangchun Cheng

Yang-han Lee

Yanglong Lu

Yangmao Wen

Yangong Zheng

Yang-wei Lin

Yang-Xin Yu

Yangyang He

Yanhu Zhang

Yanhua Luo

Yanjie Ji

Yanjie Su

Yanjie Zhou

Yanjun Yan

Yanki Aslan

Yankui Sun

Yanliang Huang

Yanlin Geng

Yanling Du

Yanmin Zhang

Yannick Salamin

Yanpeng Cao

Yanping Wang

Yanping $\mathrm{Xu}$

Yansheng Li 


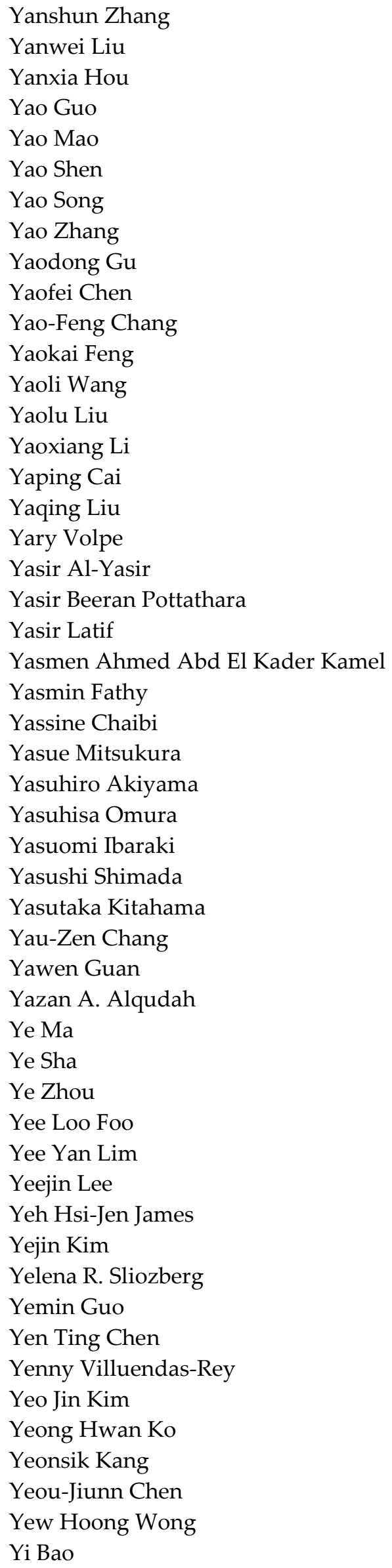

Yi Cui

Yi Fang

Yi Lei

Yi Lu

Yi Luo

Yi Qin

Yi Wang

Yi Xu

Yi Yang

Yi'nan $\mathrm{He}$

Yiannis Kantaros

Yi-Bing Lin

Yichao Zhao

Yi-Chen Chen

Yi-Chen Li

Yicheng Liao

Yichuan Deng

Yichuan $\mathrm{Wu}$

Yichun Ding

Yidong Tan

Yifan Liu

Yifan Lu

Yih Jeng

Yihai $\mathrm{He}$

Yihong Qi

Yihua Tan

Yi-Hung Liao

Yijin Pan

Yik-Chung Wu

Yi-Kuang Yen

Yi-Lang Chen

Yili Zheng

Yilong Hui

Yilun Shang

Yiming Huo

Yiming Zhu

Yin Bao

Yin Zhang

Ying Dong

Ying Fu

Ying Liu

Ying Mao

Ying Mu

Ying Tan

Ying Tie

Ying Wang

Ying-Chih Lai

Ying-Chih Liao

Ying-Hao Yu

Yingjie Xia

Yinglei Teng 


\begin{tabular}{l} 
Yingnong Chen \\
Yingpei Zeng \\
Yingpeng Sang \\
Ying-Ren Chien \\
Yingrui Shang \\
Yingtao Liu \\
Yingwei Li \\
Yingying Wang \\
Yi-Ning Wu \\
Yinji Ma \\
Yinlai Jiang \\
Yinnian Feng \\
Yinsheng Chen \\
Yin-Tsung Hwang \\
Yinwei Zhan \\
Yiran Luo \\
Yi-Ren Wang \\
Yirui Wu \\
Yishou Wang \\
Yishuo Huang \\
Yisong Tan \\
Yiting Kang \\
Yiwei Shi \\
Yixing Ding \\
Yixuan Feng \\
Yiyan Fei \\
Yiyan Li \\
Yiyang Chen \\
Yiyang Dai \\
Yi-Yung Chen \\
Yizhi Liu \\
Yizhou Zhuang \\
Yngvar Larsen \\
Yoav Linzon \\
Yogang Singh \\
Yogeenth Kumaresan \\
Yohei Kurata \\
Yohei Matsumoto \\
Yolanda Blanco-Fernández \\
Yong Gu \\
Yong Han Ahn \\
Yong Huang \\
Yong Ju Jung \\
Yong Li \\
Yong Oh Lee \\
Yong Pan \\
Yong Shang \\
Yong Soo Cho \\
Yong Wan \\
Yong Wang \\
Yong Xu \\
\hline
\end{tabular}

Yong Yang

Yong Zhang

Yong Zhao

Yong Zhu

Yongbin Liu

Yongchuan Tang

Yongcun Hao

Yongfeng Liu

Yonggang $\mathrm{Hu}$

Yonggang Xiao

Yong-Gyoo Kim

Yongho Choi

Yonghua Qu

Yong-Hwa Park

Yongjia $\mathrm{Xu}$

Yongjie Zhu

Yong-Jin Liu

Yong-Joo Chung

Yong-Joo Kim

Yongjun Zheng

Yongkai Yin

Yongli Zhu

Yongliang Qiao

Yongliang Wang

Yongming Li

Yongning $\mathrm{Wu}$

Yongqiang Hao

Yongrae Roh

Yong-Sang Kim

Yongseok Jee

Yong-Seok Jee

Yong-Seok Lee

Yongsu Park

Yongzhen Huang

Yoo Min Park

Yoon-Sik Yoo

Yoon-Soo Jang

Yooseob Song

Yo-Ping Huang

Yorgos Stephanedes

Yosef Pinhasi

Yoshiaki Adachi

Yoshifumi Saijo

Yoshihiro Fukumoto

Yosi Kristian

Yosi Shacham-Diamand

Yosoon Choi

Yosuke Aoki

Yosuke Sugioka

Youbin Zheng

Yougan Chen 
Yougang Bian

Yougang Sun

Yougen Yi

Youkou Dong

Youkyung Han

Younes Jabrane

Young Chang Jo

Young Chul Lee

Young Doo Lee

Young Eun Song

Young Min Song

Young Soo Suh

Young Sub Kwon

Youngbae Hwang

Youngchul Bae

Young-Dam Kim

Young-du Lee

Young-Duk Kim

Young-Gun Cho

Young-Hwan You

Youngjin Choi

YoungJin Jung

Youngjoon Han

Young-Ju Kim

Youngoh Lee

Youngok Kim

Young-Pil Kim

Young-Seob Jeong

Young-Sik Kim

Youngsook Bae

Youngsun Ryuh

Youngwook Seo

Yousaf Bin Zikria

Yousef Rezaei Tabar

Youssef Bichiou

Youssef Rouphael

Youwen Zhang

Youyang Qu

Youzhong Liu

Yu Cai

Yu Cao

Yu Cherepennikov

Yu Fujimoto

Yu Han

Yu Hen $\mathrm{Hu}$

Yu Kume

Yu Kyoung Ryu

Yu Li

Yu Liu

Yu Matsuda

Yu Miao
Yu Song

Yu Tak

Yu Tang

Yu Ting Tsai

Yu Tzu Wu

$\mathrm{YuWu}$

Yu Zhang

Yuan Ma

Yuan Meng

Yuan Ping

Yuan $\mathrm{Wu}$

Yuanchang Chen

Yuanchao Liu

Yuancheng Fan

Yuan-Chien Lin

Yuan-Ho Chen

Yuanjian Zhang

Yuanjie Su

Yuanjin Zheng

Yuanjing Lin

Yuankai Qi

Yuankai Wu

Yuansong Qiao

Yuanwei $\mathrm{Wu}$

Yuanxi Peng

Yuanxi Sun

Yuanxin Ye

Yuanyao Lu

Yuanyuan Jia

Yubin Guo

Yu-Bo Sheng

Yuchao Bai

$\mathrm{Yu}$-Chen Lin

Yu-Cheng Fan

Yucheng $\mathrm{Wu}$

Yu-Chi Lee

Yuchu Qin

Yu-Chun Kung

Yu-chung Hsieh

Yudhi Adhitya

Yudith Cardinale

Yudong $\mathrm{Lu}$

Yudong Shen

Yudong Zhang

Yu-Dong Zhang

Yue Cui

Yue Gu

Yue Guo

Yue Li

Yue Liao

Yue Wang 
Yue $\mathrm{Wu}$

Yuecheng Shen

Yuefeng Zhao

Yuei-An Liou

Yuejian Chen

Yuejun Kang

Yuelei Xiao

Yue-Ling Cao

Yuelong Chuang

Yuemin Yue

Yuen Clement

Yueshen $\mathrm{Wu}$

Yue-Shi Lee

Yueyun Chen

Yufei Liu

Yufei Ma

Yufeng Shi

Yuguang Fu

Yuhai Bao

Yuhan Huang

Yuhang Li

Yuhao Wang

Yuh-Chung Hu

Yuhong Li

Yuhong Liu

Yuhong Zheng

Yu-Hsi Huang

Yuhsin Hung

Yu-Hsiu Lin

Yuhuai Peng

Yuichi Fujino

Yuichi Otsuka

Yu-Jen Chen

Yuji Dong

Yuji Wada

Yujuan Si

Yujung Chen

Yukai Chen

Yu-Kai Wang

Yuki Funabora

Yuko Nakamura

Yukun Lai

Yulei Qian

Yulia Yu Bozhko

Yuliang Ma

Yuliang Shi

Yuliang Zhao

Yulong Guo

Yuming Liu

Yun Jung Heo

Yun Lin
Yun Pan

Yun Suen Pai

Yun Zhang

Yunbin Deng

Yunchao Tang

Yunchen Yang

Yuncheng Man

Yundong Li

Yunfeng Cao

Yunfeng Wu

Yung Hsiang Chen

Yung Po Tsang

Yunghui Li

Yung-Keun Kwon

Yung-Sheng Chen

Yun-ho Shin

Yunhua Rao

Yun-Ju Lee

Yunli Shao

Yunlong Guo

Yun-Lu Sun

YunMook Lim

Yun-Sik Nam

Yuntao Guo

Yunus Egi

Yun-Wei Lin

Yunxiang Wang

Yunxu Sun

Yunyoung Nam

Yunyuan Gao

Yunze He

Yuqi Fan

Yuqi Li

Yuqing Sun

Yuren Chen

Yuri D'Alessandra

Yuri Feldman

Yuri López

Yuri Rzhanov

Yuri S. Kivshar

Yuri Voznyak

Yuriy Orlov

Yuriy Povstenko

Yury Marapulets

Yury Yasyukevich

Yusen He

Yushan Sun

Yu-Shen Liu

Yu-Sheng Su

Yustus Eko Oktian

Yusuf Ozturk 


\begin{tabular}{|c|c|}
\hline Yusuf Valentino Kaneti & Zbigniew Adamiak \\
\hline Yusuke Kajiwara & Zbigniew Dziong \\
\hline Yusuke Yamada & Zbigniew Gomolka \\
\hline Yutaka Kawabe & Zbigniew J. Sroka \\
\hline Yutaka Watanobe & Zbigniew Krzemianowski \\
\hline Yu-Ting Bai & Zbigniew Kulesza \\
\hline Yuting Gao & Zbigniew Marszalek \\
\hline Yuting Zhang & Zbigniew Nadolny \\
\hline Yuvaraja Teekaraman & Zbigniew Otremba \\
\hline Yuwei Wang & Zbigniew Usarek \\
\hline Yuwen Chen & Zbigniew Zembaty \\
\hline Yuxia Duan & Zbisław Tabor \\
\hline Yuxiang Hong & Zbyněk Raida \\
\hline Yuxiang Sun & Zdenek Martinasek \\
\hline Yuxin $\mathrm{Hu}$ & Zdenek Slanina \\
\hline Yuxin Leng & Zdzisław Gosiewski \\
\hline Yuxin Xing & Ze Ji \\
\hline Yuxing Han & Zeel Maheshwari \\
\hline Yuxing Li & Zeev Vladimir Volkovich \\
\hline Yuxing Wang & Zeev Volkovich \\
\hline Yuyang Wei & Zehan Yao \\
\hline Yves Dubé & Zekra Mousavi \\
\hline Yves Gourinat & Zelei Cheng \\
\hline Yves Mahéo & Željka Cvejić \\
\hline Yves Reuland & Zeljko Hocenski \\
\hline Yvonne Tran & Željko Špoljarić \\
\hline Zabit Hameed & Zeljko Zilic \\
\hline Zacharias A. Viskadourakis & Zengsheng Chen \\
\hline Zachary Ballard & Zeno Geradts \\
\hline Zachary H. Levine & Zenon Lukaszewski \\
\hline Zafar Baloch & Zenon Nieckarz \\
\hline Zahir Hussain & Zenonas Turskis \\
\hline Zahir M. Hussain & Zesong Fei \\
\hline Zahra Abbasi & Zeyang Zhou \\
\hline Zahra Kazemi & Zhan Gao \\
\hline Zahra Pooranian & Zhan Song \\
\hline Zaid Alyasseri & Zhan Wang \\
\hline Zaidao Wen & Zhangjie Liu \\
\hline Zaifeng Shi & Zhangjun $\mathrm{Yu}$ \\
\hline Zain Ul-Abdin & Zhangyong Li \\
\hline Zain-Aldeen A. Rahman & Zhanhui Wang \\
\hline Zakaria Meliani & Zhanjun Wu \\
\hline Zakariya Oraibi & Zhanlin Ji \\
\hline Zaloa Sanchez Varela & Zhanna A. Boeva \\
\hline Zan Li & Zhanping Song \\
\hline Zaneta Swiderska-Chadaj & Zhan-Yi Hu \\
\hline Zao Yi & Zhao Cheng He \\
\hline Zaojun Fang & Zhao Ren \\
\hline Zara Bagdasarian & Zhao Xu \\
\hline Zavala-Rivera Paul & Zhaogang Shu \\
\hline Zbignevs Marcinkevics & Zhaohui Li \\
\hline
\end{tabular}


Zhaojie Luo

Zhaoqian Xie

Zhaoqiang Chu

Zhaowei Kong

Zhaowei Zhang

Zhaowen Yan

Zhaoyang Ding

Zhaoye Qin

Zhaozheng $\mathrm{Hu}$

Zhaozong Meng

Zhe Chen

Zhe Shen

Zhe Sun

Zheguang Zou

Zhen Qiu

Zhen Wang

Zhen Wu

Zhen Xu

Zhen Zhang

Zhenbin Zhang

Zhenfeng Gong

Zhenfeng Shao

Zheng (Eddie) Li

Zheng Bin

Zheng Cui

Zheng Fan

Zheng Ji

Zheng Kuangyu

Zheng Li

Zheng Qian

Zheng Tong

Zheng Wang

Zhengfang Wang

Zhenggan Zhou

Zhengkuan Lee

Zhengliang Zhu

Zhenglin Li

Zhengqiang Wang

Zhenguang Liu

Zhengwei Li

Zhengxiang Shi

Zhengxing Sun

Zhenhu Jin

Zhenhua Yu

Zhenhua Zou

Zhenhuan Yi

Zhenhui Du

Zhenkun Lei

Zhenling Ma

Zhenlong $\mathrm{Xu}$

Zhenming Peng
Zhenping Guan

Zhenxiang Yi

Zhenxin Wang

Zhenxin Zhang

Zhenxing Gao

Zhenyang Ding

Zhenyin Hai

Zhenyu Li

Zhenzhan Wang

Zhenzhi Lin

Zhenzhong $\mathrm{Hu}$

Zhenzhou Wang

Zheyuan Chen

Zhezhuang Xu

Zhi Ning

Zhibin Sun

Zhibin Wang

Zhicheng Cui

Zhicheng Qiu

Zhidong Xue

Zhifang Liang

Zhifang Pan

Zhifeng Tang

Zhifu Liu

Zhigang Li

Zhiguang Tang

Zhiguo Zhang

Zhihai Qiu

Zhihong Deng

Zhihong Yao

Zhihua Xiong

Zhihua Xu

Zhihua Yu

Zhihui Zeng

Zhihuo Xu

ZhiJun Yan

Zhikang Li

Zhiling Hou

Zhilu Lai

Zhimeng Yin

Zhiming Zhang

Zhiping Liu

Zhiqian Chen

Zhiqiang $\mathrm{Hu}$

Zhiqiang Liang

Zhiqiang $\mathrm{Xu}$

Zhiqin Zhu

Zhiqun $\mathrm{Hu}$

Zhisheng Gao

Zhitao Xiao

Zhitian Liu 
Zhiwei He

Zhiwei Mao

Zhiwei Zhao

Zhixi Nie

Zhiyi Tang

Zhiyi Wu

Zhiyong Liu

Zhiyong Zhang

Zhiyong Zhou

Zhiyu Jiang

Zhiyuan Feng

Zhiyuan Ren

Zhiyuan Yu

Zhiyuan Zhu

Zhizhong Ding

Zhong Su

Zhongbao Wei

Zhongfeng Wang

Zhonghao Li

Zhonghua Xue

Zhongli Wang

Zhongmin Jin

Zhongmin Qiu

Zhongmin Xiao

Zhongqi Liu

Zhongxiang Wei

Zhongyi Hu

Zhou Xu

Zhou Zhang

Zhoutao Zheng

Zhouyi Wang

Zhu Sun

Zhu Xiao

Zhuang Cheng

Zhuangqiang Gao

Zhuangzhi Sun

Zhuhua Hu

Zhuofu Liu

Zhuoqi Cheng

Zia Ullah

Zicheng Chi

Zichun Zhong

Žiga Gosar

Zigang Deng

Zihan Kan

Zijia Li

Zijia Zhong
Zijian Qiao

Zijian Wang

Zijie Zhu

Zijing Wong

Zijun Wang

Zilong Zhang

Zilu Liang

Zimi Sawacha

Zinaida M. Kuznetsova

Zine El Abiddine Fellah

Zipei Fan

Ziping Liu

Zitong Yu

Ziv Yaniv

Živana Ninčević-Gladan

Ziyue Zeng

Zlatin Zlatev

Zofia Rzepecka

Zoheir Khademian

Zois Boukouvalas

Zoka Milan

Zöldy Máté

Zoltán Bálint

Zoltán Gillay

Zoltan Juhasz

Zoltán Kolláth

Zoltan Rozsa

Zongjie Wang

Zongmei Gao

Zongsong Gan

Zongyin Yang

Zoran Minic

Zoran Perić

Zoran Salcic

Zoran Stamenkovic

Zouhair Hanani

Zsolt Gulácsi

Zsolt Molnár

Zuguang Ying

Zuojun Liu

Zuo-Min Tsai

Zuomin Zhao

Zuqing Zhu

Zvi Roth

Zvonko Radosavljevic

Zygmunt Mikno 